DEPARTAMENTO DE QUÍMICA

ÁrEA DE QUÍMICA ANALÍTICA

\title{
DEVELOPMENT OF ELECTROCHEMICAL BIOSENSORS AND SENSORS FOR THE DETERMINATION OF INTEREST ANALYTES
}

Hugo Cunha da Silva

Tesis Doctoral

Burgos, 2019 



\section{UNIVERSIDAD DE BURGOS}

DePartamento de Química

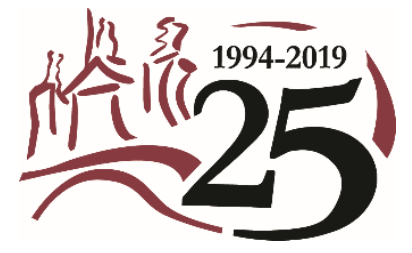

Memoria de investigación titulada "Development of electrochemical biosensors and sensors for the determination of interest analytes" presentada para optar al grado de doctor en la Universidad de Burgos.

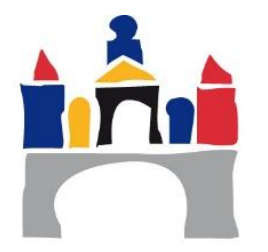

Fdo.: Hugo Cunha da Silva 



\section{UNIVERSIDAD DE BURGOS}

\section{DEPARTAMENTO DE QUíMICA}

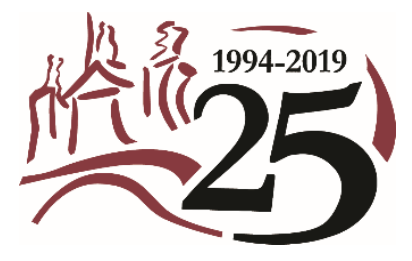

Dña. M. Julia Arcos Martínez, Catedrática de Universidad del Departamento de Química de la Universidad de Burgos,

INFORMA FAVORABLEMENTE sobre la presente Tesis Doctoral, titulada "Development of electrochemical biosensors and sensors for the determination of interest analytes", realizada en el Área de Química Analítica del Departamento de Química de la Universidad de Burgos por Don Hugo Flávio Cunha da Silva.

Y para que así conste, firma el presente informe en Burgos, a 2 de abril de 2019.

Fdo.: M. Julia Arcos Martínez 

Dedicated to the memory of my mom 

I would like to express my acknowledgments to all those people and institutions that, in one way or another, have been present in the process and realization of this $\mathrm{PhD}$ thesis.

First of all, I would like to thank to my supervisor, Professor María Julia Arcos Martínez, for the professional and personal support she gave me, before and during my journey in the University of Burgos. For the confidence she has put on me to integrate this project, the scientific guidance, enthusiasm, dedication and availability. Also, I would like to thank her for transmitting me her passion for research and to keep me positive when everything seemed to go wrong. Her extraordinary perseverance is also evident in this thesis.

Second, I cannot forget the Professor Ana Cristina Dias Cabral, who brought me to the University of Burgos for the first time, and who introduced me to the world of electrochemical sensors. For her availability, scientific guidance and professionalism. If she had not believed in me, I certainly would not have developed this work. Thank you.

For the warm way he welcomed me during my stay in his laboratory at the University Libre de Bruxelles, I would like to acknowledge to Professor Jean-Michel Kauffman. Thank you for all the knowledge transmitted and for allowing me to experience this enriching work.

I would like to thank to all the researchers, personnel and professors of the analytical chemistry department, who were always available for constructive discussions and exchange of ideas.

Research can be very ungrateful and there are periods when difficulties limit the progress of the work. In these moments the companionship and the support that I had from my lab mates was priceless. For the support in those difficult hours and for the incredible friendship, I would like to leave my most sincere thanks to all who have accompanied me during my thesis, in especially to Bego and Lydia. I cannot forget Ana, Carla, Laura and Sarah, who were often the third and fourth hands of support, and who have been without doubt, fundamental to the progress of the work. 
A huge thanks to my family, for your love, advices, recommendations and unconditional support. Thank you!

I want to thank the people who crossed my path, and who in one way or another, were and are a great support for me. For their invaluable friendship and support, I own a special "Muito obrigado" to Ander, Chegão, Claudia and Micha. Thank you for all the incredible support, joy and companionship. I extend my acknowledgments to all my friends who were also important during this journey.

Finally, I would like to acknowledge to the Spanish Ministry of Economy, Industry and Competitiveness, for the funding obtained through an FPI grant, and also, for the mobility grants, that allowed me to carry out the two academic research internships in foreign research centers.

Thank you! 


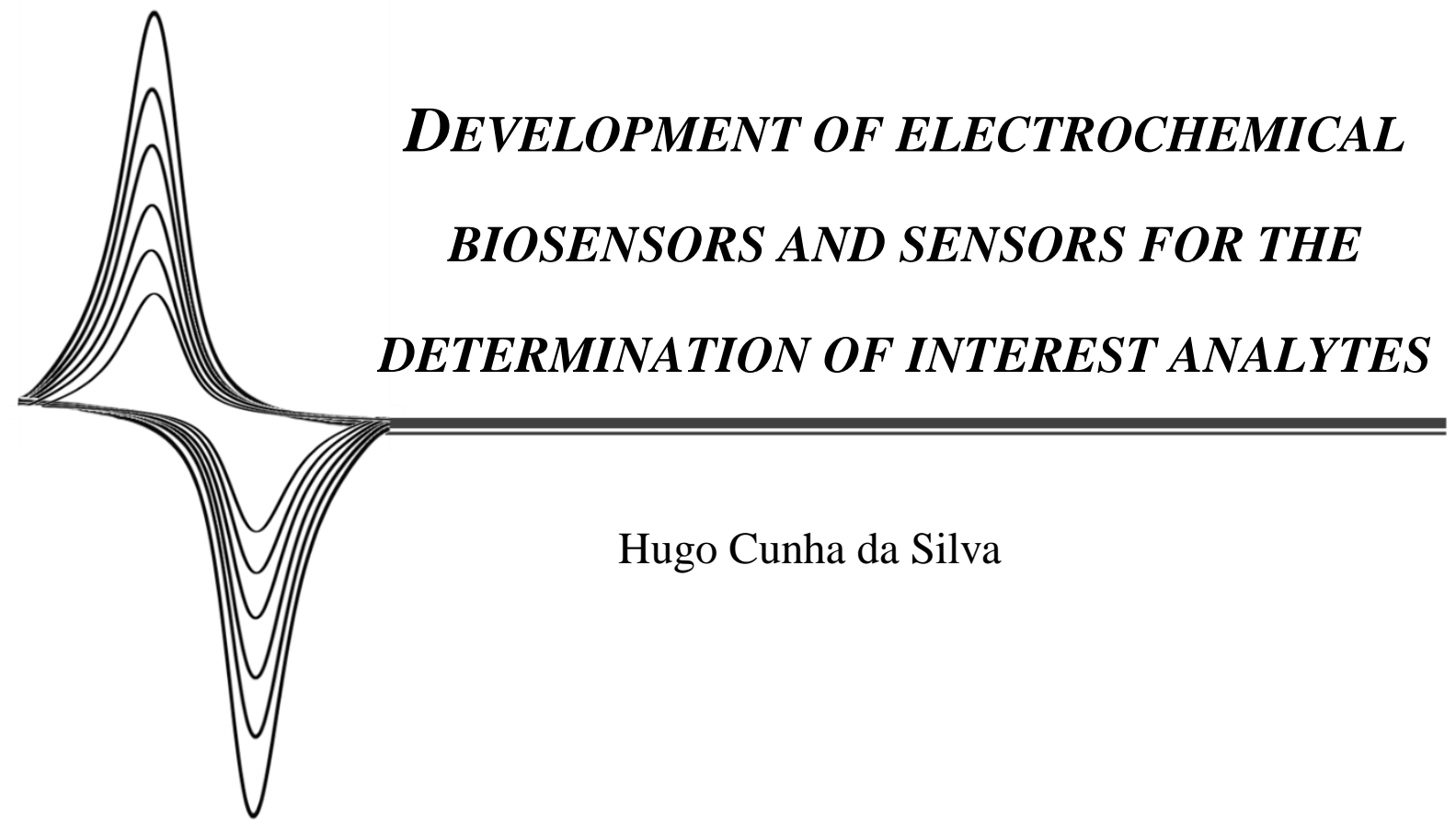





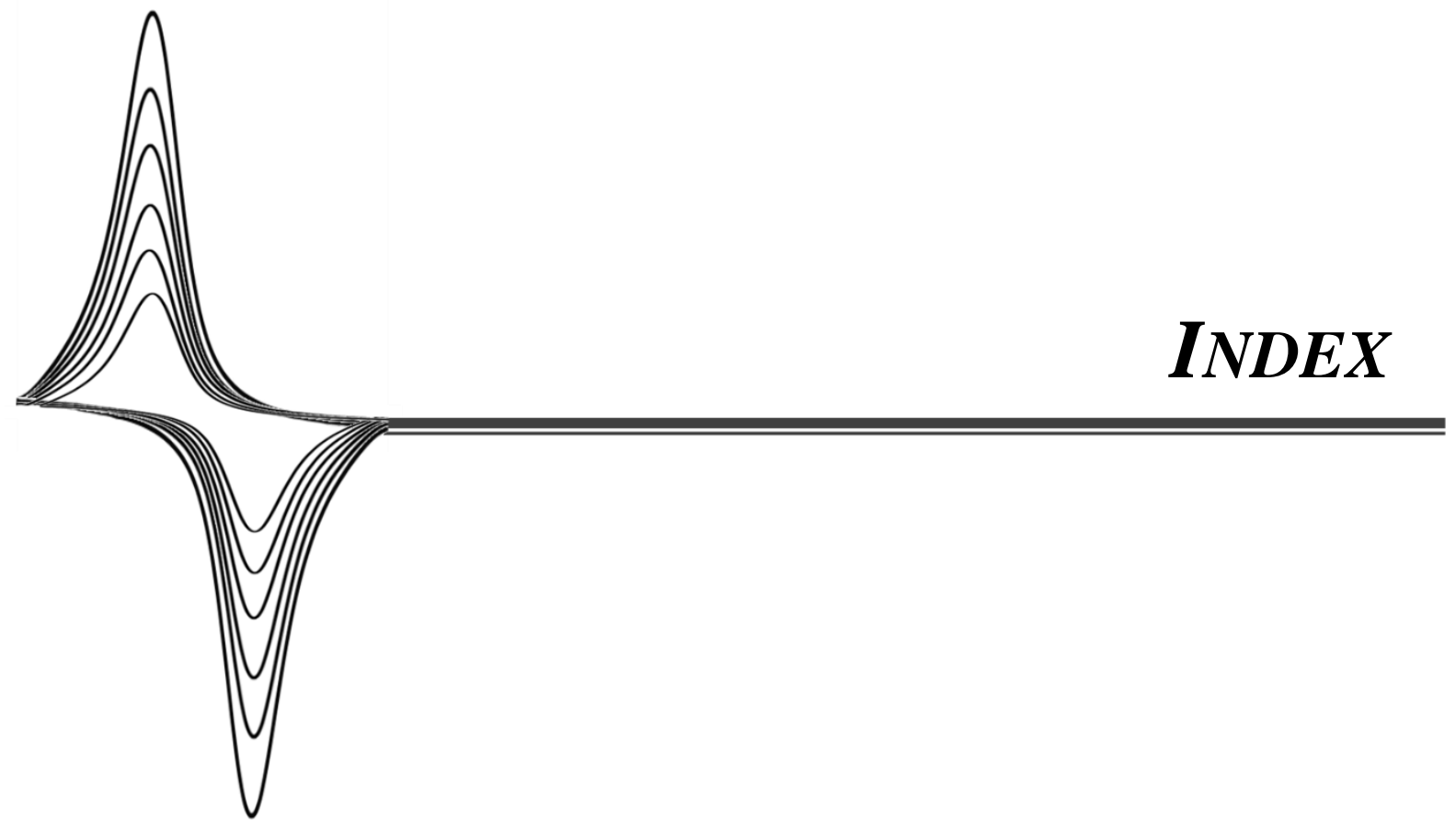





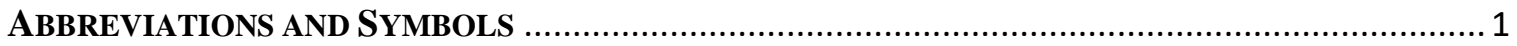

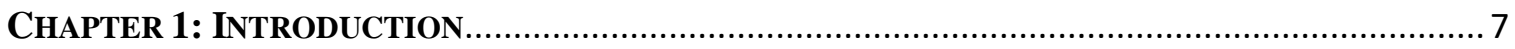

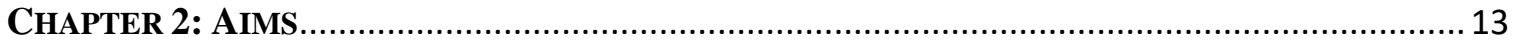

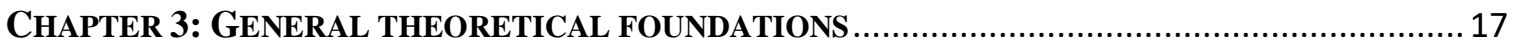

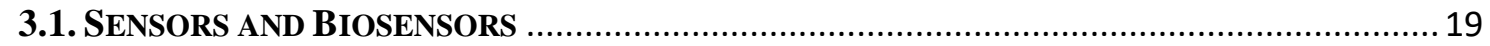

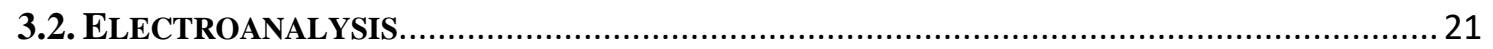

3.3. CONTROLLED-POTENTIAL ELECTROANALYTICAL EXPERIMENTS .................................. 22

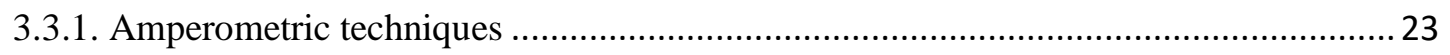

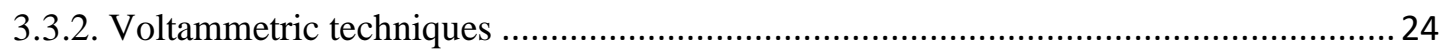

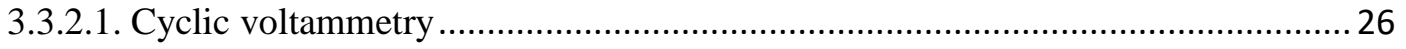

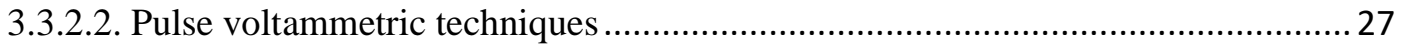

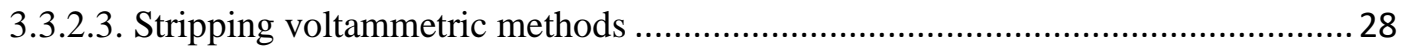

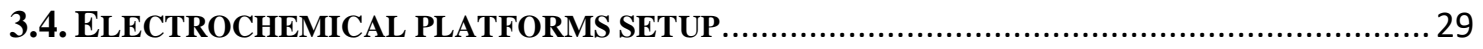

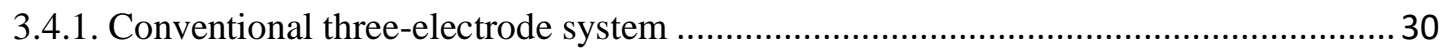

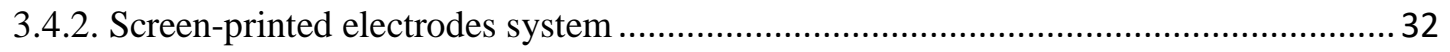

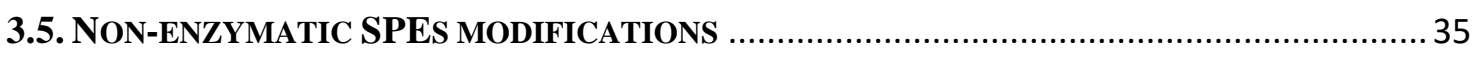

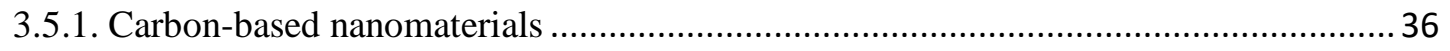

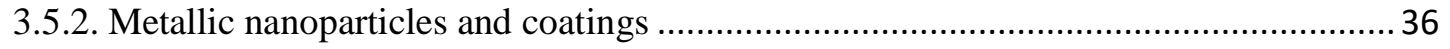

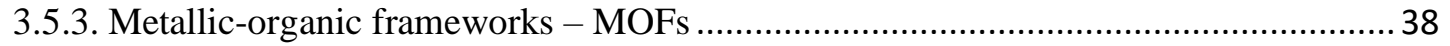

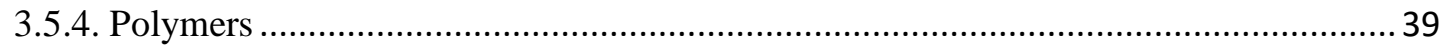

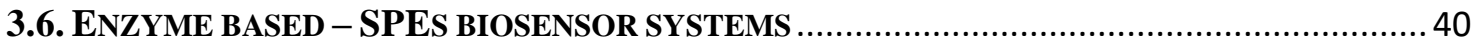

3.6.1. Enzymes as biorecognition elements in biosensing strategies .................................. 41

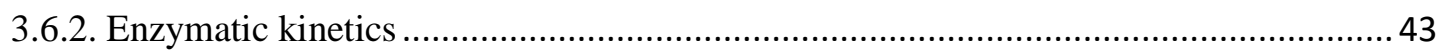

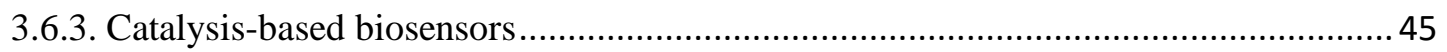

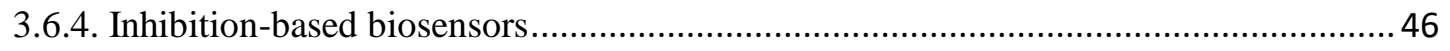

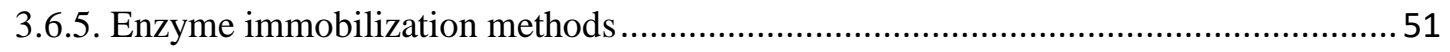

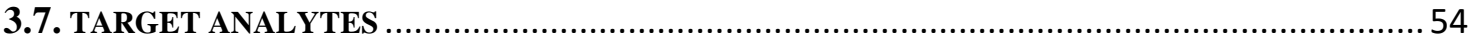

3.7.1. LACTATE

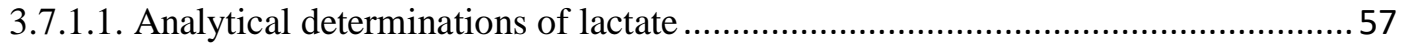

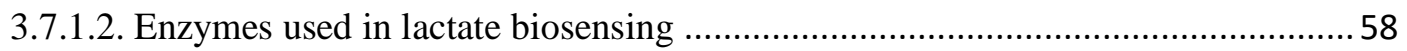

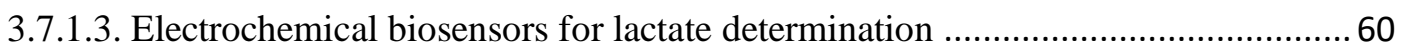




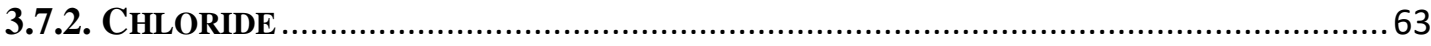

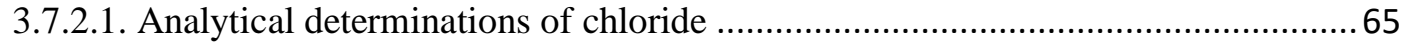

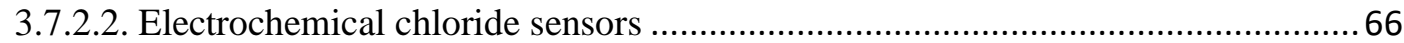

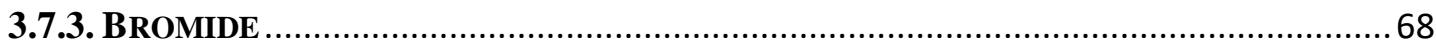

3.7.3.1. Analytical methods for bromide determinations .............................................. 70

3.7.3.2. Electrochemical approaches for bromide determination.................................... 71

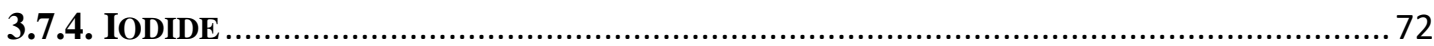

3.7.4.1. Analytical determination of iodide .............................................................. 74

3.7.4.2. Electrochemical determinations of iodide ...................................................... 76

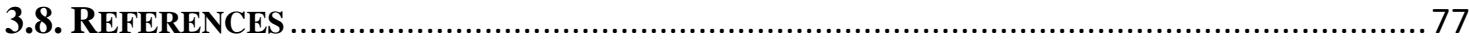

CHAPTER 4: AMPEROMETRIC BIOSENSOR FOR LACTATE ANALYSIS............................... 125

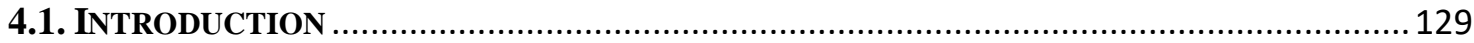

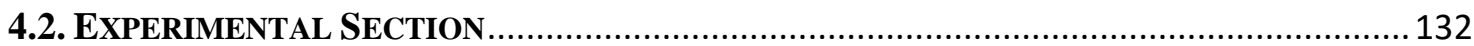

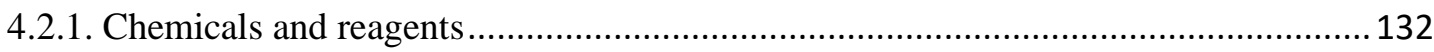

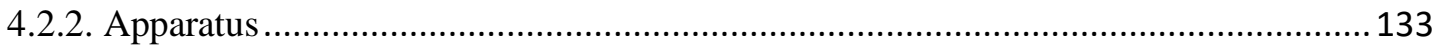

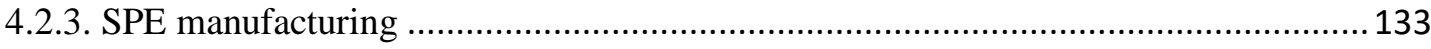

4.2.4. Electrode Modification …............................................................................. 134

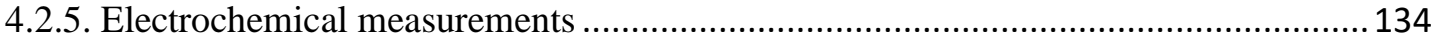

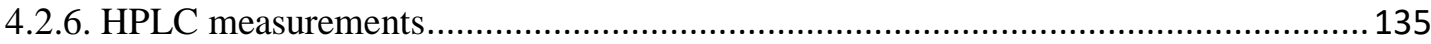

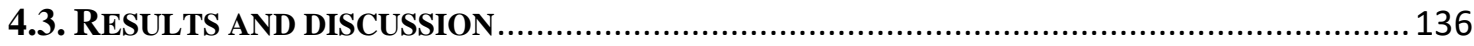

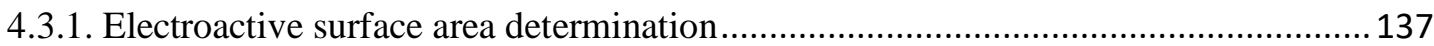

4.3.2. Chitosan influence in amperometric measurements .............................................. 140

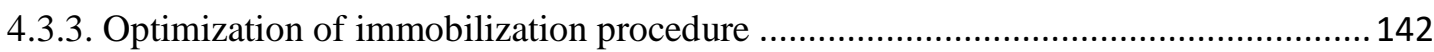

4.3.3.1. Copper metal-organic framework influence in amperometric measurement ...... 142

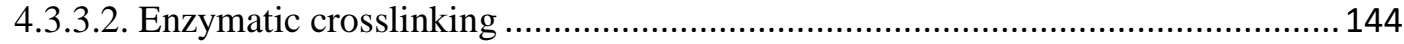

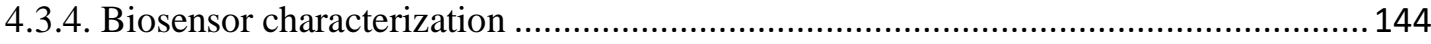

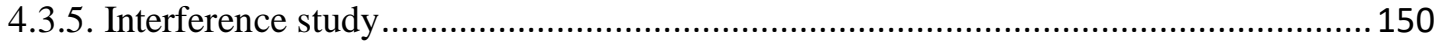

4.3.6. Application in biological and food samples ...................................................... 153

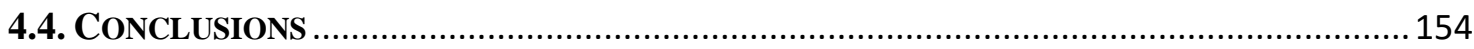

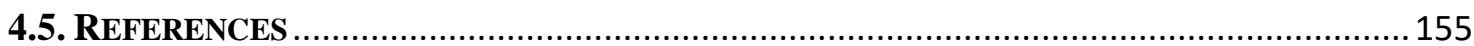

CHAPTER 5: LACTATE OXIDASE INHIBITION - A PH-DEPENDENT MECHANISM ..................... 165

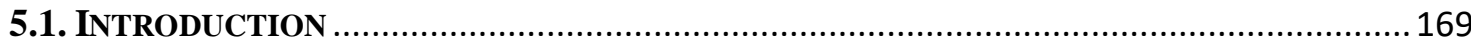




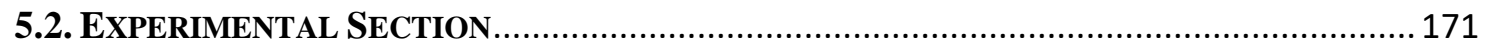

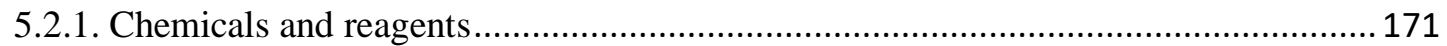

5.2.2. Screen-printed carbon electrodes (SPCE) manufacturing ....................................... 172

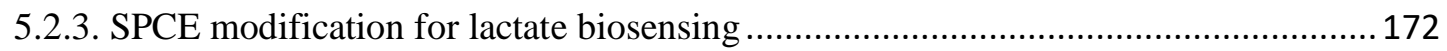

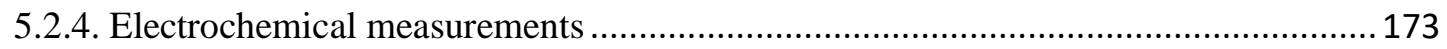

5.2.5. Fluorescence spectroscopy measurements ......................................................... 173

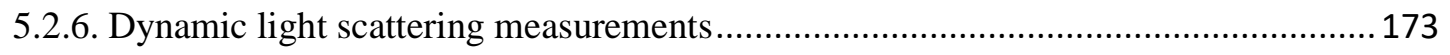

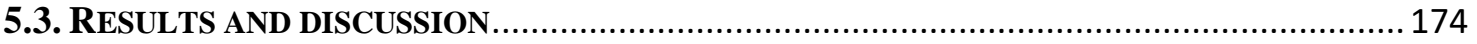

5.3.1. Determination of crosslinked LOx kinetic parameters ............................................ 174

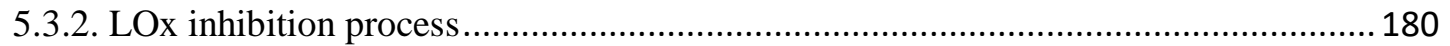

5.3.3. LOx structural changes promoted by substrate ....................................................... 183

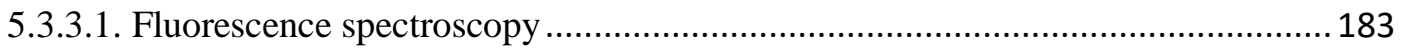

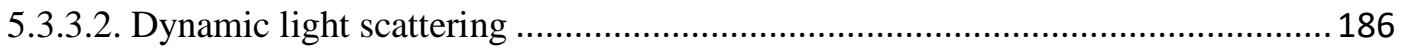

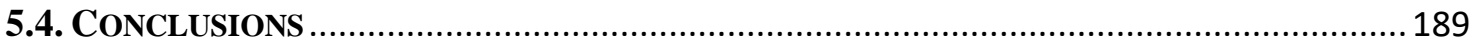

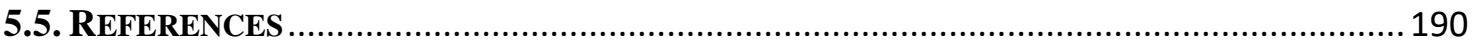

CHAPTER 6: CHLORIDE SENSING PLATFORM USING A SPPTE .......................................... 195

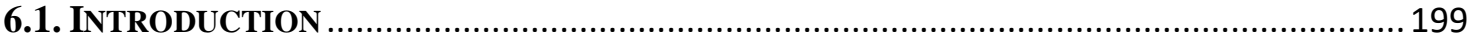

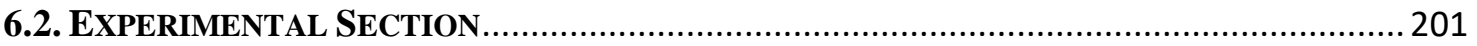

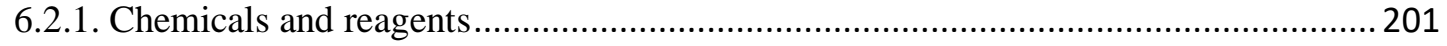

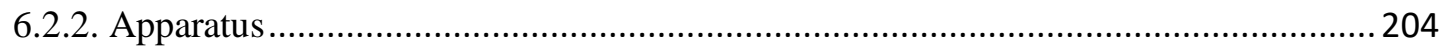

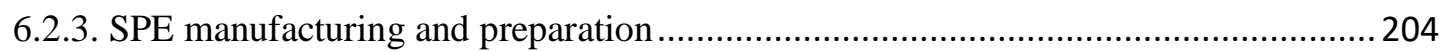

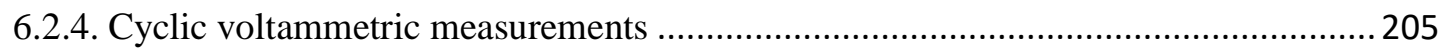

6.2.5. Cathodic stripping voltammetry for chloride determination.................................... 205

6.2.6. Chloride ion selective electrode measurement ........................................................ 206

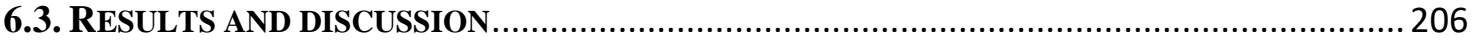

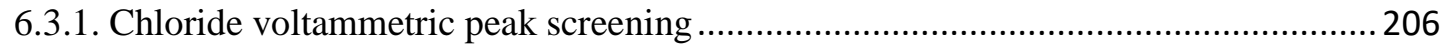

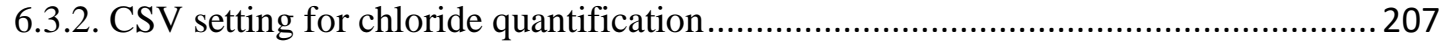

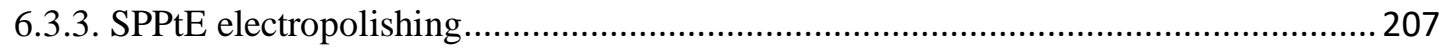

6.3.4. Electrochemical mechanism for CSV chloride reduction peak ................................ 213

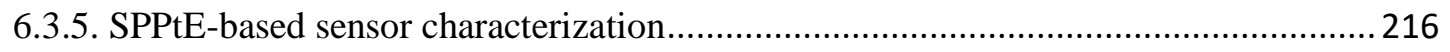

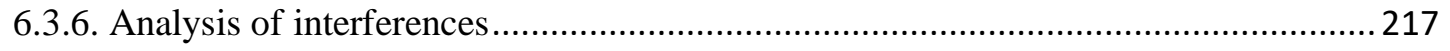

6.3.7. SPPtE-based sensor application and validation ................................................... 218 


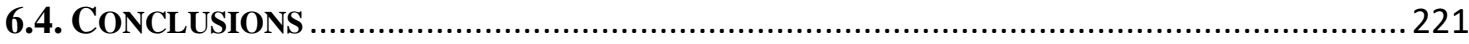

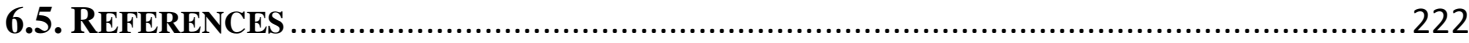

CHAPTER 7: RHODIUM NANOPARTICLE-BASED SENSOR FOR BROMIDE DETERMINATION .... 231

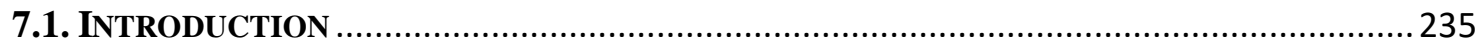

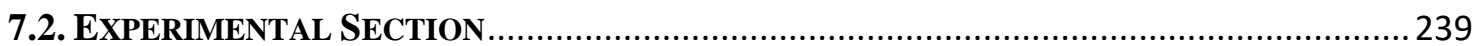

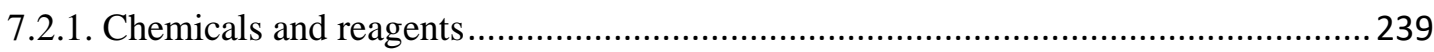

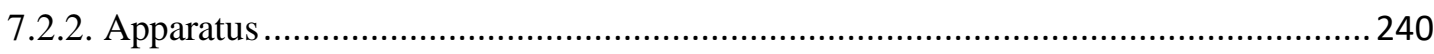

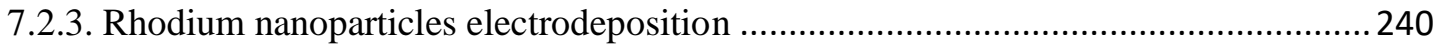

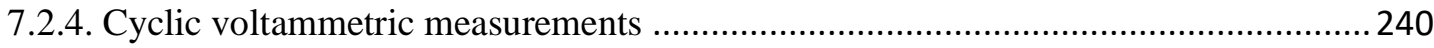

7.2.5. Cathodic stripping voltammetry (CSV) for bromide determination ......................... 240

7.2.6. Methanolic extraction for dextromethorphan hydrobromide formulation ................. 241

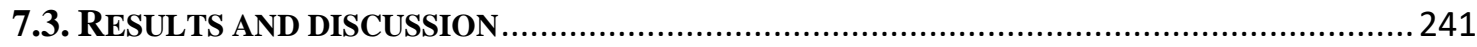

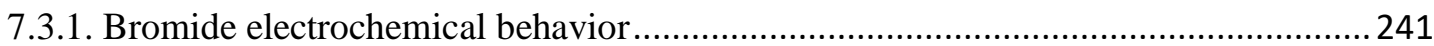

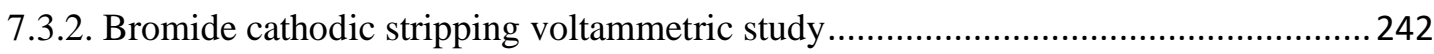

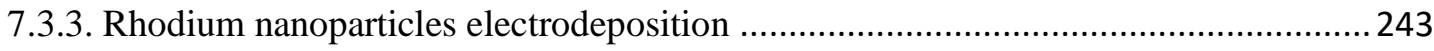

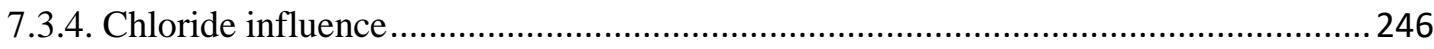

7.3.5. Characterization of Rh/SPCE-based sensor for bromide determination ....................247

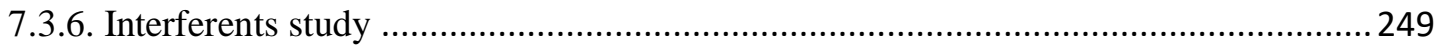

7.3.7. Bromide determination in real-life samples ......................................................... 251

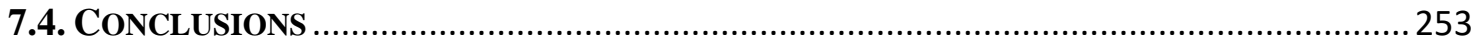

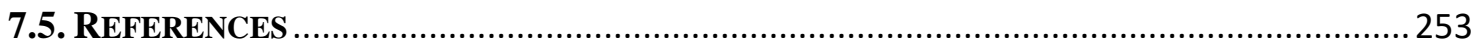

CHAPTER 8: DISPOSABLE SENSORS FOR DETERMINATION OF IODIDE ..................................... 259

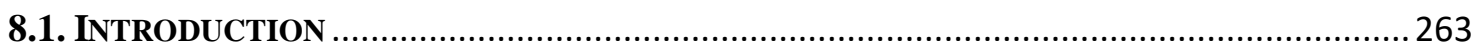

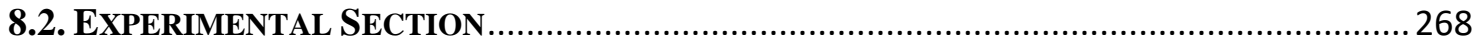

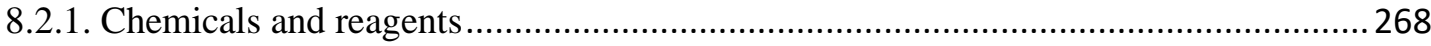

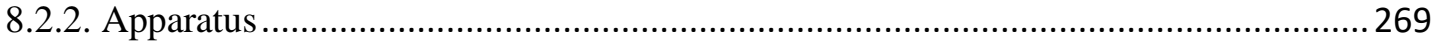

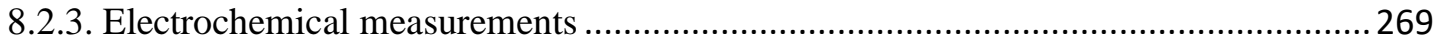

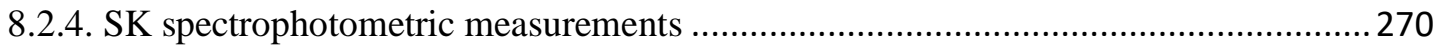

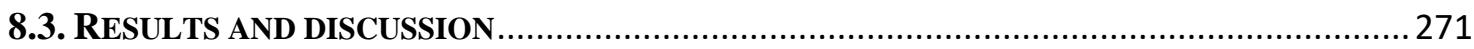

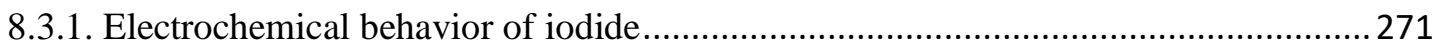

8.3.2. CSV for a selective determination of iodide ............................................................ 272

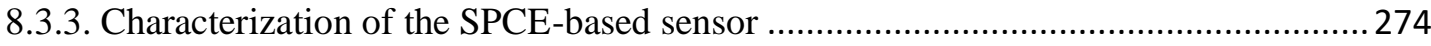


8.3.4. Construction of CS/SPCE for iodide determination ............................................... 276

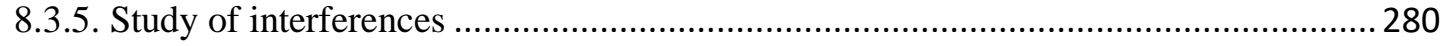

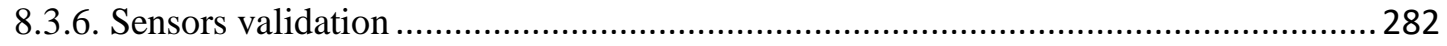

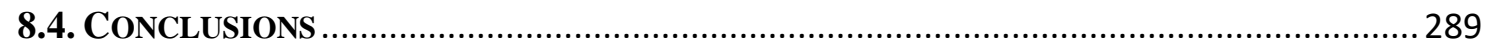

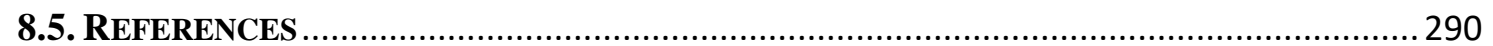

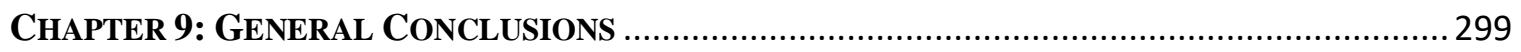

ANNEX: TYROSINASE MICROREACTOR-DETECTOR: BENZOIC ACID INHIBITION.................... 305

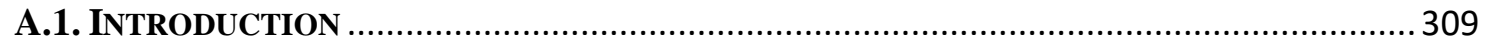

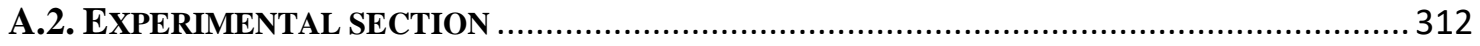

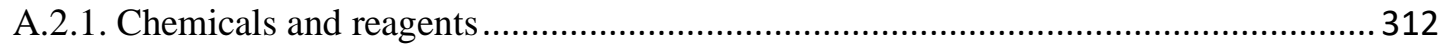

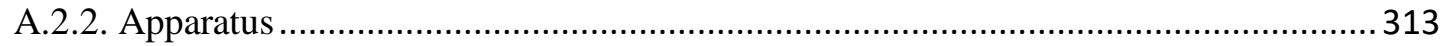

A.2.3. Tyrosinase microreactor-detector preparation ...................................................... 313

A.2.4. Experimental parameters influencing the response …........................................... 315

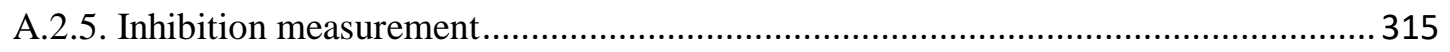

A.2.6. Determination of the enzyme recovery rate......................................................... 316

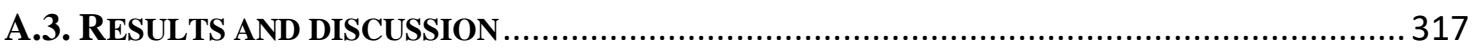

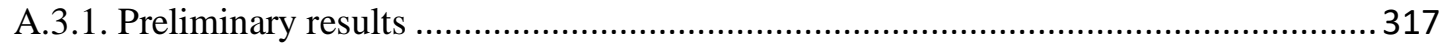

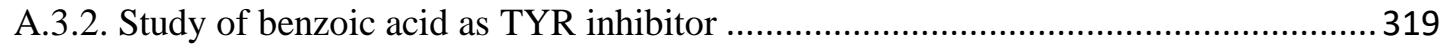

A.3.3. Inhibitor potency and $\mathrm{IC}_{50}$ determination for benzoic acid .................................... 320

A.3.4. TYR reactivation after benzoic acid inhibition ..................................................... 322

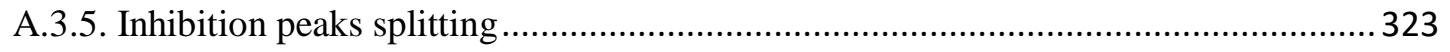

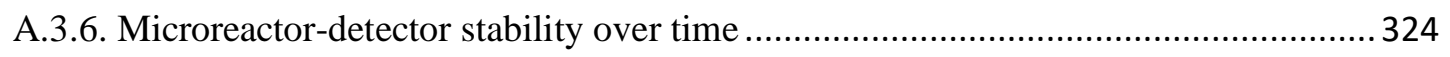

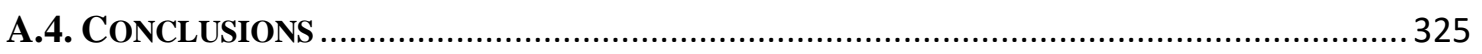

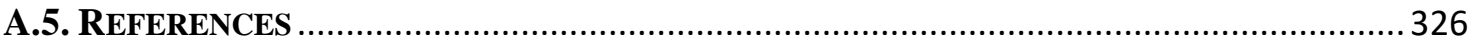





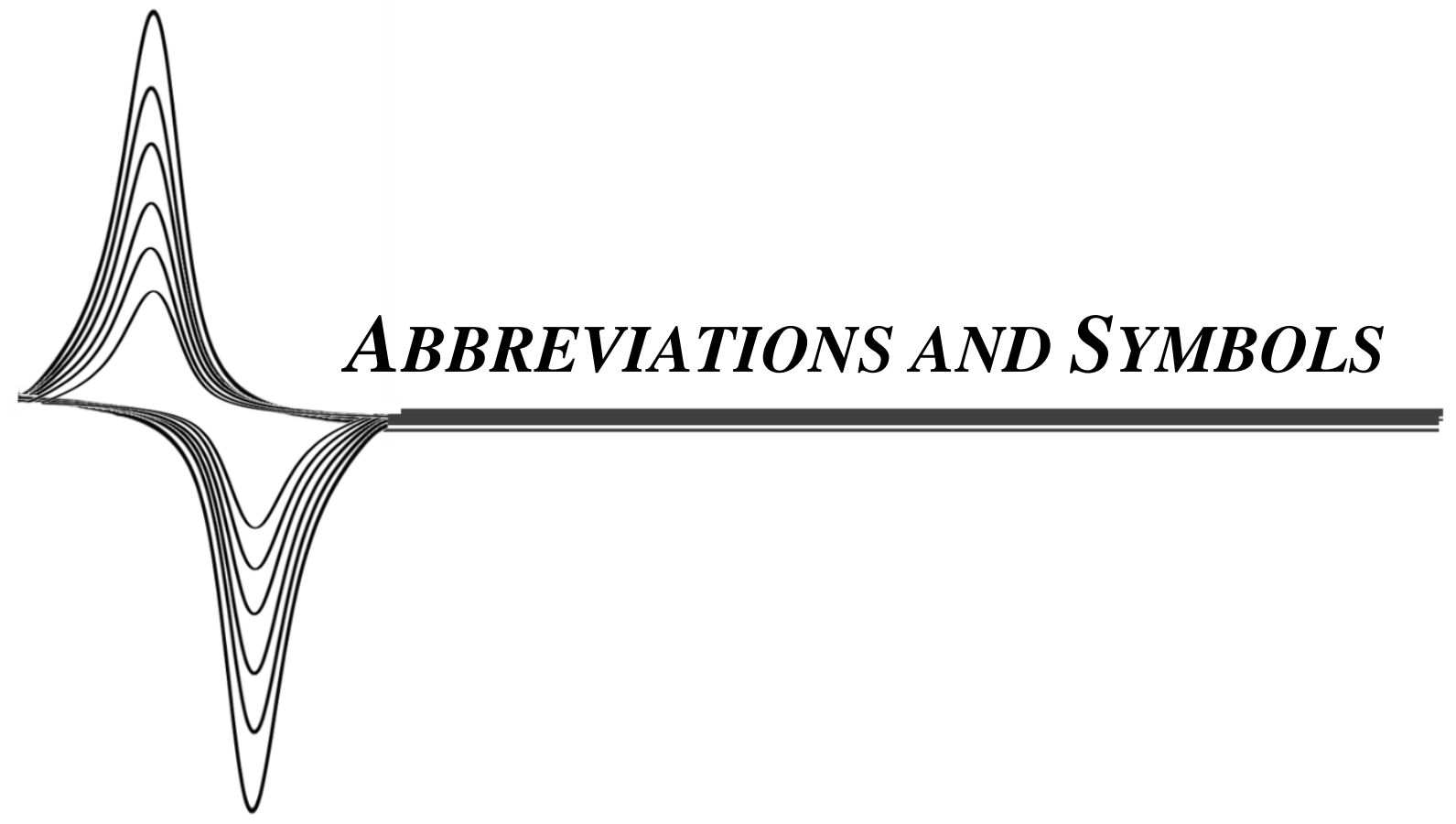





\begin{tabular}{|c|c|}
\hline Abbreviation/Symbol & Full Name \\
\hline $\bar{A}$ & Electrode area \\
\hline$\overline{A_{0}}$ & Average signal of enzymatic activity in absence of inhibitor \\
\hline $\mathrm{Ag} / \mathrm{AgCl}$ & Silver/silver chloride reference electrode \\
\hline Ag-Nps & Silver nanoparticles \\
\hline$A_{i}$ & Signal of enzymatic activity in presence of inhibitor \\
\hline ASV & Anodic stripping voltammetry \\
\hline Au-Nps & Gold nanoparticles \\
\hline$b$ & Average slope of calibration curves \\
\hline $\mathrm{BA}$ & Benzoic Acid \\
\hline $\mathrm{Br}-\mathrm{ISE}$ & Bromide ion selective electrode \\
\hline BSA & Bovine serum albumin \\
\hline$C$ & Concentration \\
\hline $\mathrm{CE}$ & Counter electrode \\
\hline $\mathrm{CF}$ & Cystic fibrosis \\
\hline CFTR & Cystic fibrosis transmembrane conductance regulator \\
\hline $\mathrm{Cl}$ - ISE & Chloride ion selective electrode \\
\hline CS & Chitosan \\
\hline CSV & Cathodic stripping voltammetry \\
\hline CTAB & Hexadecyltrimethylammonium bromide \\
\hline $\mathrm{Cu}-\mathrm{MOF}$ & Copper Metal-Organic framework \\
\hline $\mathrm{CV}$ & Cyclic voltammetry \\
\hline CYST & Cysteamine \\
\hline$D$ & Diffusion coefficient \\
\hline DB & Detecting block \\
\hline DLS & Dynamic light scattering \\
\hline DPV & Differential pulse voltammetry \\
\hline DQ & Dopaquinone \\
\hline E & Enzyme \\
\hline
\end{tabular}




$\begin{array}{cl}E_{\text {app. }} & \text { Applied potential } \\ E_{\text {begin }} & \text { Start potential } \\ E_{\text {dep }} & \text { Deposition potential } \\ E_{\text {end }} & \text { End potential } \\ E_{p} & \text { Peak potential } \\ E P & \text { Electropolishing step } \\ E S & \text { Enzyme-substrate complex } \\ \text { ESA } & \text { Electroactive surface area } \\ E S S & \text { Enzyme-substrate inactive complex } \\ E_{v t x l} \text { and } E_{v t r 2} & \text { Cyclic voltammetry potential vertices } \\ \text { FIA } & \text { Flow injection analysis system } \\ \text { GA } & \text { Glutaraldehyde } \\ \text { GCE } & \text { Glassy carbon electrode } \\ \text { HRP } & \text { Horseradish Peroxidase } \\ i & \text { Current } \\ \text { I- ISE } & \text { Iodide ion selective electrode } \\ I(\%) & \text { Percentage of enzyme inhibition } \\ I C_{50} & \text { Inhibitor concentration that decrease 50\% of enzyme activity } \\ I C_{70} & \text { Inhibitor concentration that decrease 70\% of enzyme activity } \\ \text { IDD } & \text { Iodide deficiency diseases } \\ i_{\text {max }} & \text { Current at maximum rate of the enzymatic reaction } \\ i_{p} & \text { Peak current } \\ i_{p a n o d i c} & \text { Anodic peak current } \\ i_{p \text { cathodic }} & \text { Cathodic peak current } \\ \text { ISE } & \text { Ion-selective electrode } \\ \text { KA } & \text { Kojic Acid } \\ K_{I} & \text { Inhibition constant - affinity of enzyme to the inhibitor } \\ K_{M} & \text { Michaelis-Menten constant } \\ K_{M}^{\prime} & \text { Inhibition Michaelis-Menten constant } \\ \text { LOD } & \text { Limit of detection } \\ & \end{array}$




\begin{tabular}{|c|c|}
\hline $\mathrm{LO}_{\mathrm{x}}$ & Lactate oxidase \\
\hline LSV & Linear sweep voltammetry \\
\hline M-Nps & Metallic nanoparticles \\
\hline MOF & Metal-Organic framework \\
\hline$n$ & Electron stoichiometry \\
\hline NADH or NAD+ & Nicotinamide adenine dinucleotides \\
\hline$P$ & Enzymatic products \\
\hline PB & Phosphate buffer \\
\hline PBS & Phosphate buffer saline \\
\hline PET & Polyester films \\
\hline $\mathrm{Pt} / \mathrm{SPCE}$ & Screen-printed carbon electrode modified with Pt \\
\hline $\mathrm{PtO}$ & Platinum oxide \\
\hline$Q_{o}$ & Charge associated with surface oxidation \\
\hline $\mathrm{RE}$ & Reference electrode \\
\hline Rh-Nps & Rhodium nanoparticles \\
\hline RSD & Residual standard deviation \\
\hline$S$ & Enzymatic substrates \\
\hline$[S]$ & Enzymatic substrate concentration \\
\hline SAM & Self-assembled monolayer \\
\hline SEM & Scanning electron microscopy \\
\hline SK & Sandell-Kolthoff reaction \\
\hline$\left[S_{\max }\right]$ & Substrate concentration after which inhibition process begins \\
\hline SPAgE & Screen-printed silver electrode \\
\hline SPCE & Screen-printed carbon electrode \\
\hline SPE & Screen-printed electrode \\
\hline $\mathrm{SPE} \mathrm{Ag} / \mathrm{AgCl}$ & Screen-printed silver/silver chloride pseudo reference electrode \\
\hline SPPtE & Screen-printed platinum electrode \\
\hline$S_{x / y}$ & Blank or smallest concentration detected standard deviation \\
\hline$t_{d e p}$ & Deposition time \\
\hline$t_{\text {rest }}$ & Equilibration time \\
\hline
\end{tabular}




$\begin{array}{cl}\text { TYR } & \text { Tyrosinase } \\ \text { TSH } & \text { Thyroid stimulating hormone } \\ \text { UI } & \text { Urinary Iodide } \\ V & \text { Rate of the enzymatic reaction } \\ v & \text { Scan rate } \\ V_{\max } & \text { Maximum rate of the enzymatic reaction } \\ \text { WE } & \text { Working electrode } \\ \text { WHO } & \text { World Health Organization }\end{array}$




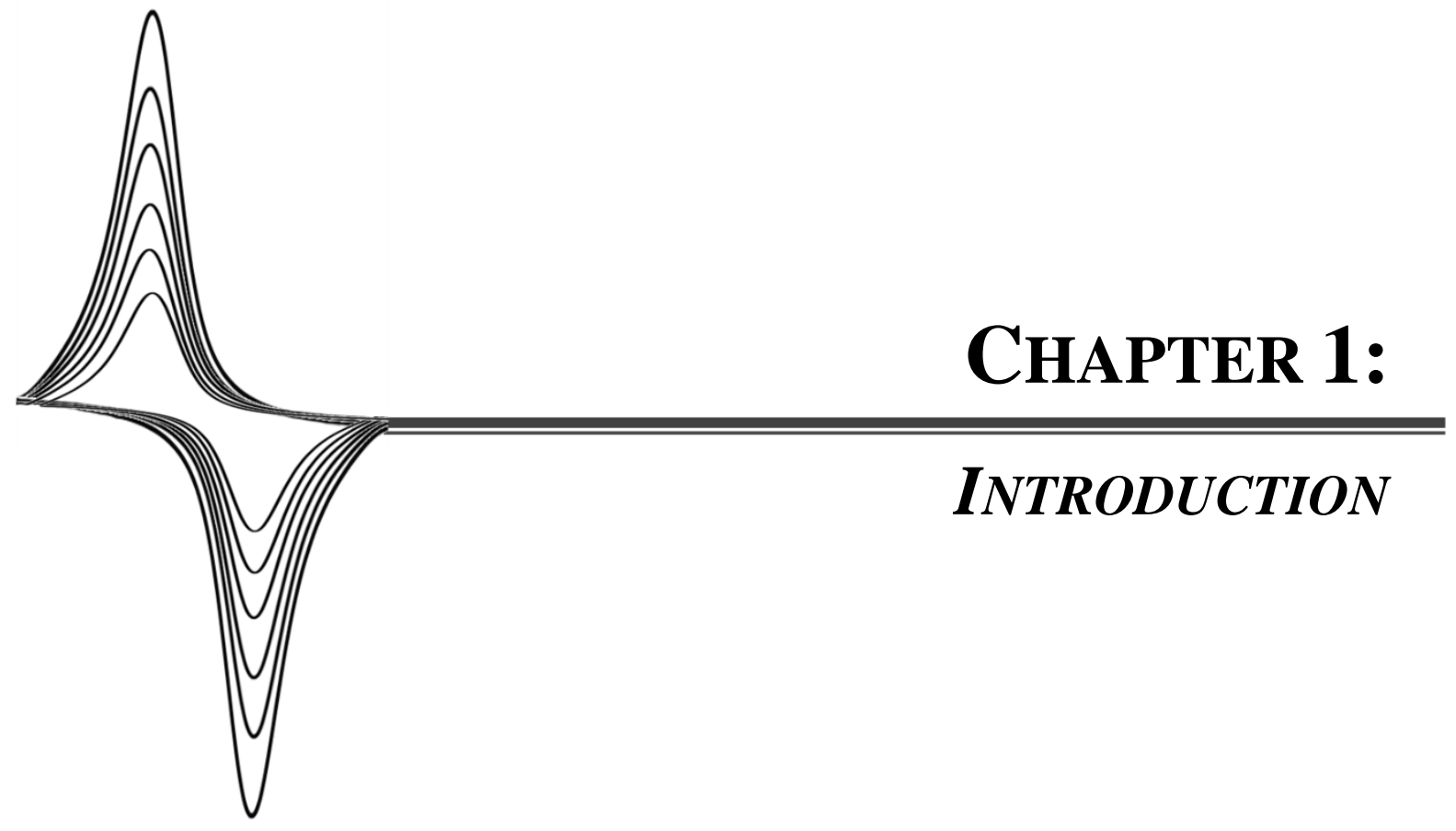



Analytical chemistry is a science that comprises much research fields as techniques and methods, aiming the qualitative and/or quantitative determination of interest analytes. It is useful in the most important fields of chemistry and in many other scientific areas, since they benefit directly from the development of novel methods and instruments.

In the laboratory, we contact daily with the devices responsible for the remarkable and continuous advances on analytical field. While this area has brought important advances, also add some difficulties. Many procedures require several steps of samples preparation, and highly sophisticated, specialized and expensive apparatus. Also, expertise personnel and the adequate knowledge are required to obtain significant results, turning such advances unpractical for routine use.

Electrochemical techniques overcome many of such problematic, receiving a major attention as promising analytical tools. Moreover, the versatility, low-cost, small sized and portability for in situ applications, gathered by the screen-printed technology, added important advantages to the field. Additionally, the simplicity to carry out electrode modifications, allows to manipulate the screen-printed electrodes (SPEs) to achieve a suitable selectivity for the interest analytes determination.

Taking advantages of the use of SPEs as efficient analytical tools, the initial work presented herein, pursued the aims enclosed in the research project entitled "Screen printing of electrochemical sensors and biosensors on flexible substrates. Development of electronic prototype devices" (TEC2013-40561-P), which financed this work. The focus of this project was the development of electrochemical SPEs-based sensors and biosensors to monitoring interest analytes in sweat samples. Therefore, after defining the aims of the work (Chapter 2), in the chapter 3 are described the general theoretical foundations, in which the electroanalytical techniques setup, and also, some modifications used for the development of electrochemical sensors and biosensors are collected. Additionally, part of this chapter is dedicated to the importance and the state of the art of the determination of the target analytes explored in this thesis. 
During the last decade, the increased interest in lactate as biomarker molecule for numerous conditions, and also, as indicator of athletes' physical performance, highlighted this organic acid as an ideal target for a noninvasive sweat analysis. Therefore, chapter 4 illustrate the development and application of an amperometric lactate biosensor based on the lactate oxidase enzyme. This biosensor was applied to sweat samples, but also to other biological matrices and food products. The device exhibited a dual-range response, as a consequence of an atypical substrate inhibition mechanism, which affected the enzyme activity at high concentrations of lactate.

In consequence, in chapter 5 are gathered the results obtained during an academic internship in the University of Beira Interior (Portugal). During the stay, the research focus was the study of lactate oxidase structural changes promoted at inhibitory substrate levels; by searching for the formation of protein aggregates and evaluating the outcomes in the enzymatic activity registered at the electrochemical biosensor.

The results obtained in these two chapters defined the course and the focus of the rest of the work, since some analytical difficulties were found when halide anions are present in the matrices.

In particular, the presence of chloride anions lead to difficulties in determining lactate. Taking advantage of the behavior found at platinum surfaces and concerning the significance of chloride in sweat analysis, in chapter 6 it is described a new approach for voltammetric determination of this anion. A simple, selective and reusable platinum SPEbased sensor is proposed as alternative method for chloride determination in sweat, but also, in food, pharmaceutical and different biological samples. Sweat chloride was considered as a major focus, once is the main indicator of cystic fibrosis disease. Although it was not a problem in the samples evaluated in this chapter, the platinum SPE-based device, showed susceptibility to false positive results in presence of high bromide concentrations.

The increased interest for bromide determination, noticeable with the number of publications released over the last 5 years, and also, the behavior found for this anion in 
chapter 6, give rise to the work presented in chapter 7. The modification of the SPEs with rhodium nanoparticles, allowed to obtain a highly selective bromide sensor, that was validated for environmental and pharmaceutical analyses.

Next, chapter 8 presents the selective determination of iodide in biological and pharmaceutical samples, using two SPEs-based sensors. One approach relied in the control of the voltammetric stripping conditions, while the other was based in the electrode modification with a chitosan coating. This modification allows to obtain analytical levels for the trace determination of iodide anions. Both devices showed particular applicability in biomedical determinations of the iodide.

Finally, in Annex are summarized the results obtained during an academic internship in the University Libre de Bruxelles (Belgium), which research was centered in enzymatic inhibition studies, using an amperometric bioreactor in a flow injection configuration. 



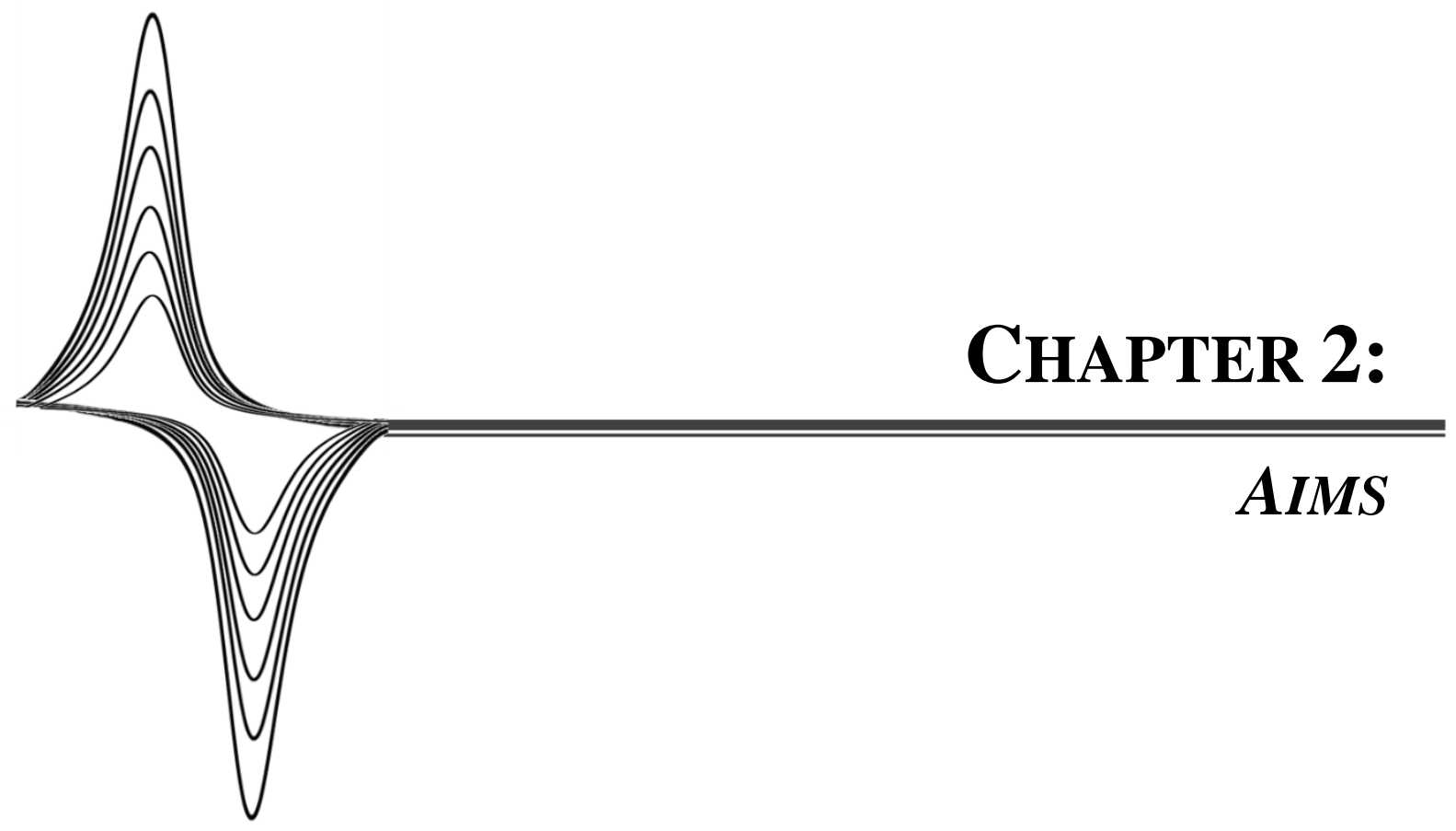



The main goal of the research presented herein, is the development of new electrochemical biosensors and sensors for the determination of different compounds with interest in the analytical field.

The use of screen-printed electrodes (SPEs) as the base for sensors and biosensors development, offers highly adaptable platforms, that add new possibilities of miniaturization and modifications, improving the monitoring of interest compounds. The proposed sensing tools, must present advantages to the routine techniques used on determinations, meeting a low-cost, reproducible, sensitive, specific and an in situ analysis. Relating to these hints, the main goal of this $\mathrm{PhD}$ thesis implies the achievement of specific objectives, which are here described:

- Screening of the interest analytes electrochemical behavior.

- Improving the electrochemical signals, to achieve higher sensitivities and selectivity for the target analytes.

- Functionalization of SPEs with nanomaterials, biorecognition elements (enzymes), among others, which implies the study of different modification strategies.

- Optimization of the experimental parameters and variables that strongly influence the electrochemical response.

- Validation of the proposed sensors in terms of:

I. Precision, defining sensors' reproducibility and repeatability;

II. Limit of detection for the interest analytes;

III. Accuracy, comparing the results obtained with standard analytical techniques;

IV. Viability, applying the sensors to real samples analysis.

In addition to these specific objectives, in this work are also contemplated complete studies of the inhibition mechanisms found for the enzymes used in the biosensing approaches. 



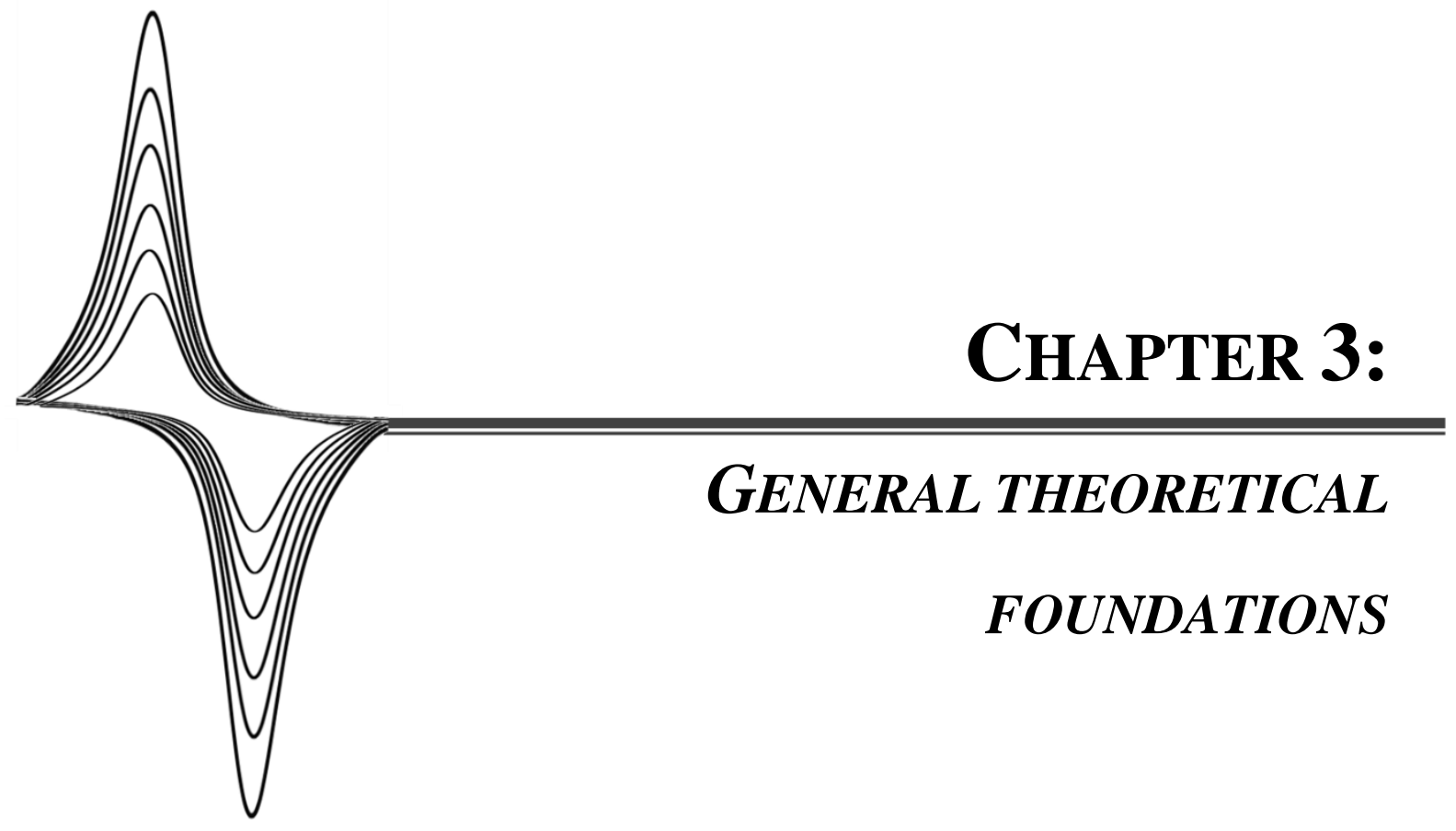





\subsection{SENSORS AND BIOSENSORS}

Nowadays, the call for effective and simple analytical techniques, converted the development of sensors and biosensors in one of the most active areas in the analytical research. The large market for these products has encouraged numerous applications in diverse fields, such as pharmaceutical, environmental, agroalimentar, as well as, in medical sectors [1-3].

IUPAC definition of a chemical sensor relies in a device that transforms information originated from a chemical reaction related with interest species, or from a physical property of the system, into a useful analytical signal. A sensor must contain a receptor component that generate the information of interest, which is transferred to the transducer component, the part that convert such information into an analytical signal [4]. Finally, a third main component based on computer-assisted mathematical modeling, should describe the process that occurs at the sensor, processing the signal to the human operator [4-7].

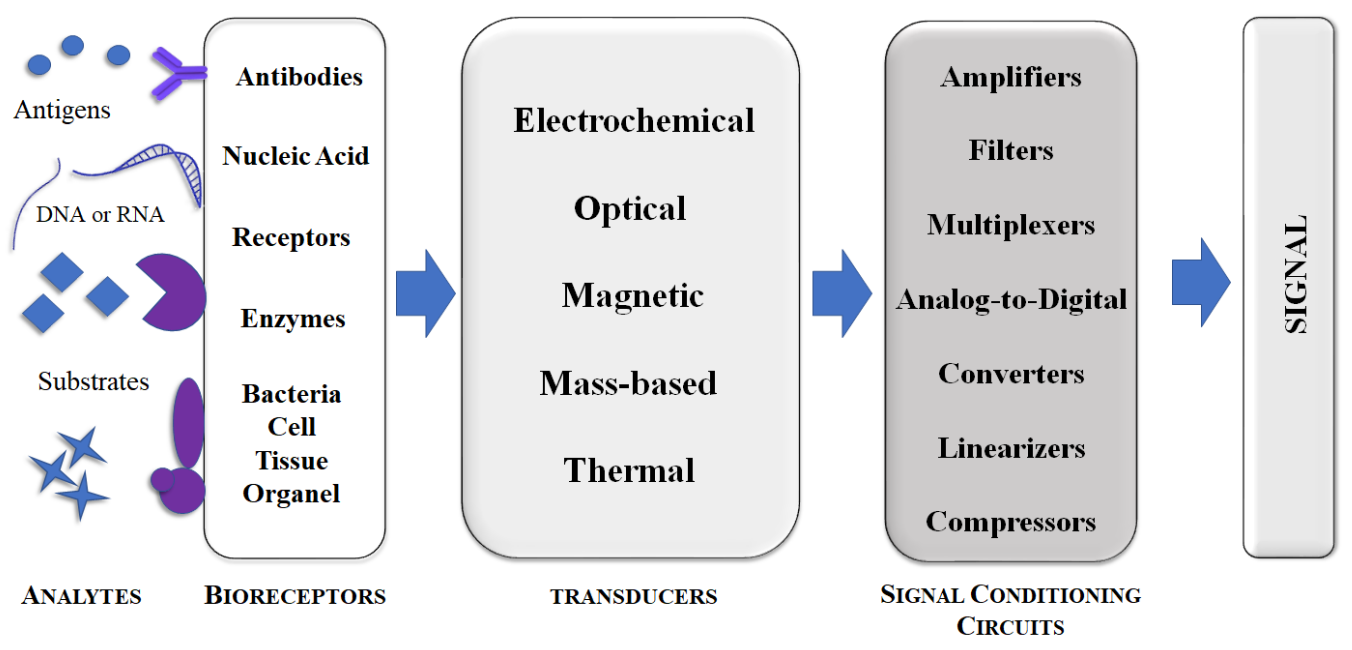

Figure 3.1. Schematic representation of the components of a biosensor device. 
One of the best known chemical sensors, is the glass electrode for the $\mathrm{pH}$ determination, which indicates the activity of the hydrogen ion in solution [1]. However, as shown in figure 3.1 , the analytical relevance of numerous types of target analytes (the molecule intended to identify and quantify) and the very distinct strategies for transducing, produce a vast number of sensing approaches possibilities $[1,4,5]$. Moreover, the wide range of available receptors, allowed to incorporate biological recognition elements, mostly enzymes, leading to the generally recognized as biosensor. This terminology can be adapted as "affinity sensor", when antibodies, nucleic acids or other non-catalytic biomolecules, are used as bioreceptors [8].

The bioreceptors, are usually the responsible component of the biosensor devices' selectivity, giving a response based upon various principles:

(i) Physical, with no chemical reactions involved;

(ii) Chemical, in which a chemical reaction with participation of the analyte, gives rise to the analytical signal.

(iii) Biochemical, in which a biochemical process is the source of the analytical signal.

An "ideal" sensor should display (i) a great selectivity, avoiding interferences in the samples; (ii) a signal proportionality with the quantity or quality of the input variation, i.e. interest analyte concentration; (iii) should offer a rapid analysis; and also, (iv) great sensitivity of response. Additionally, especial important when using biorecognition elements, an "ideal" biosensor should also present $(v)$ a great stability, offering a large storage and shell-time, and (vi) a reusable character to minimize the preparation and analyses costs $[7,9]$.

The challenge to construct a device able to offer all these features, give rise to the development of several strategies with applicability in very different fields. Among all the existing possibilities for signal transducing, the ones based in electrochemical techniques, offer advantages in terms of analysis time, simplicity and specificity of response. The electrochemical transducers are the most used, playing an important role for the advances of the sensing field [1,2,7,9]. Therefore, in following chapters are introduced and 
overviewed the fundamental terms and concepts employed in the development of this $\mathrm{PhD}$ thesis.

\subsection{ElECTROANALYSIS}

Electrochemistry, based on electrons transfer from one reactant to another, i.e. oxidation/reduction reactions [10], born in 1800, with Alessandro Volta inventing the first version of the modern electrical battery, the "voltaic pile". Later in the year 1834, Michael Faraday advance the two laws of electrochemistry, predicting the process known as electrolysis, in which determine the quantity of products, resulting from passing a certain amount of current through a chemical compound or its solution [11]. From then to now, uncountable advances in the electrochemical field occurred, including new electrode materials, that have been applied as useful tools in the analytical field, originating a new discipline - Electroanalysis.

Electroanalytical techniques, deal with electrical magnitudes (current, potential or charges), studying their relation with chemical processes [2]. The use of such properties for analytical purposes, create a vast range of possibilities and applications in very distinct areas $[2,10]$.

In the most of electrochemical experiments, the process takes place at the electrode-solution interface, requiring at least two electrodes and a solution, comprising the scheme called electrochemical cell. One of the electrodes, the working electrode (WE), responds to the target analyte; while the second one, termed the reference electrode (RE), is of a constant potential independent of solution properties. Also, a third probe, termed counter electrode (CE), can be found in electrochemical cells. The CE made of an inert conducting material, helps to pass the current flowing through the cell to the WE, functioning as a currentcarrying auxiliary electrode. Typically, no processes of analytical interest occur at the CE surface $[2,7,10-12]$. Electrochemical cells can be classified according with the requirement or the production of electric energy, as respectively, electrolytic or galvanic cells [2,11]. 
The type of electrical signal used for the quantification, can be obtained through a potentiometric measurement (static technique - zero current), in which the information about sample composition is obtained from the equilibrium potential established across a membrane. The major applications for potentiometry is the monitoring of ionic species such as protons, halides, calcium, potassium, among others $[2,10,11]$.

Other important group of electroanalytical experiments, is based on dynamic processes of charge transfer (non-zero-current) at the electrode-solution interface, where a potential or an intensity function is applied, respectively, potentiostatic or galvanostatic techniques, and the response of the system registered [2,10,11]. These group, present higher sensitivity, selectivity, and wide linear ranges. Moreover, the portable and low-cost instrumentation, speciation capability and the extensive variety of electrodes, allows to work in unusual

environments [2]. Additionally, some of the potentiostatic techniques achieve extremely low detection limits, with reports of nano- [13] , pico- $[14,15]$ and femtomolar [16] determinations performed in small volumes $(5-20 \mu \mathrm{L})$, which open doors to new applications [2].

\subsection{CONTROLLED-POTENTIAL ELECTROANALYTICAL EXPERIMENTS}

The objective of controlled-potential experiments is to obtain a current response related to the concentration of a target analyte, monitoring the transfer of electron(s) during the redox process (equation 3.1).

$$
O+n e^{-} \leftrightarrow R
$$

where $\mathrm{O}$ and $\mathrm{R}$, are respectively the oxidized and reduced forms of the redox couple. The total current obtained, is the sum of the faradaic current, resulting from the sample redox chemical reactions; and the nonfaradaic charging background current, which results from the process not-related with the electrochemical reaction $[2,12]$. 
In following sections, are briefly described the main techniques used to perform controlledpotential experiments.

\subsubsection{Amperometric techniques}

Amperometry or amperometric detection, is the simplest but also the widely technique used in electrochemical systems $[2,7,12,17]$. Indeed, many electroanalytical approaches require this technique' properties [2]. The measurement is carried out by applying a constant potential $\left(E_{\text {app. }}\right)$ and monitoring the resulting current-time dependence, which reflects the change in the analyte concentration at the vicinity of the WE surface (Figure 3.2).

Variations of the technique can be found regarding the supporting electrolyte conditions, that can be $(i)$ hydrodynamic for solutions under motion (convection or flow), or (ii) static conditions (diffusion), as the case of a sample drop or a quiescent solution [2,10-12]. The latter, usually performed under a constant analyte concentration, is commonly referred as chronoamperometry (Figure 3.2. A). This technique is closed related with the Cottrell equation, which describes the current-time dependence for a diffusion controlled process at planar electrodes (equation 3.2).

$$
i=\frac{n F A D^{\frac{1}{2}} C}{\sqrt{\pi t}}
$$

where, $i$ is the current, $n$ is the stoichiometric number of electrons involved in the reaction, $F$ is the Faraday's constant, $A$ is the electrode area, $D$ is the diffusion coefficient, $C$ is the electroactive species' concentration and $t$ the time. The application of a $E_{a p p}$ at the WE, leads to the adjustment of the double-layer charging to the new voltage, promoting the fast decay of the current intensity. This process is only significant during the initial period of the experiments. However, if the $E_{a p p}$ is enough to promote redox processes of the electroactive species, the depleting of such species can be defined as function of $t^{1 / 2}$ $[7,10,11,18]$. 
In case of stirred batch (hydrodynamic conditions), is observed the generation of current levels (Figure 3.2. B) corresponding with the analyte additions. Also, the technique is valuable in flow injection analysis (FIA), resulting in current peaks, instead of current levels (Figure 3.2. C). Therefore, the obtained currents can be used to quantify the target analyte concentration in the samples, using a previously determined calibration factor $[2,10,11]$.
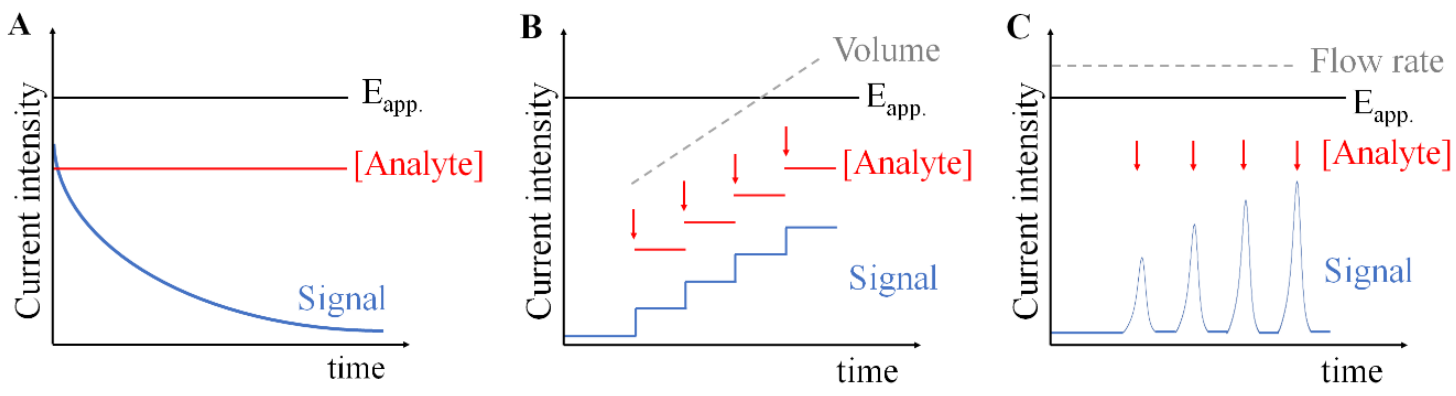

Figure 3.2. Typical signal of amperometric detection at: (A) static solution; (B) stirred batch system and (c) FIA system. Variation of current intensity (signal) with analyte concentration ([Analyte]), added or injected (arrows), measured at fixed applied potential ( $\left.E_{a p p .}\right)$.

\subsubsection{Voltammetric techniques}

Contrary to the amperometry, in voltammetric measurements a potential ramp is imposed, resulting in the current output vs potential. These techniques are usually performed in quiescent solutions [2,10-12].

In presence of electroactive species, the potential sweep originates voltammetric peaks, containing quantitative and qualitative information about the redox processes. The peaks' potential can be used as qualitative information about the compounds, since is dependent of the redox groups' character. Indeed, these techniques are the most widely used for acquiring qualitative data about electrochemical processes. On the other hand, the 
quantification of voltammetric peaks in terms of current, area or charge, can be related with the concentration of electroactive species in the solution [2,10-12].

Quantitative and kinetic information of the voltammetric processes can be obtained through the use of Randles-Sevcik equation, which is a tool that describes the effect of the scan rate on the peak current $[10,18-21]$. The simplified Randles-Sevcik model at $25^{\circ} \mathrm{C}$, results in equation 3.3 .

$$
i_{p}=\left(2.96 \times 10^{5}\right) n^{3 / 2} A C D^{1 / 2} v^{1 / 2}
$$

where, $i_{p}$ is the peak current, $n$ is the electron stoichiometry, $A$ the electrode area $\left(\mathrm{cm}^{2}\right), C$ the concentration of electroactive species $\left(\mathrm{mol} / \mathrm{cm}^{3}\right), D$ the diffusion coefficient of the electroactive species $\left(\mathrm{cm}^{2} / \mathrm{s}\right)$, and $v$ the potential scan rate $(\mathrm{V} / \mathrm{s})$. Using the relationships defined by this equation and the known experimental parameters, the $D, A$ or $n$ can be determined mathematically $[10,18-21]$.

According with equation 3.3, the current is directly proportional to $C$ and increases with $v^{1 / 2}$, indicative of a mass transport controlled reaction (diffusion) [2]. Therefore, at constant $C$ value, linear plots of $i_{p}$ vs $v^{1 / 2}$, should pass through the origin and have a gradient, from which the diffusion coefficient may be estimated [12]. Moreover, species where the $D$ is known (or can be estimated), the slope of the $i_{p}$ vs $v^{1 / 2}$ plots, provide information into the stoichiometry of the redox process [18] or $A$ [22]. Sometimes, a non-linear relation of $i_{p}$ vs $v^{1 / 2}$ can be also found, which indicate a non-diffusional controlled process, with the possible involvement of adsorbed intermediates.

The simplest technique for potential variation is the linear sweep voltammetry (LSV), which is based in a single potential ramp changing with the time, from a start potential $\left(E_{\text {begin }}\right)$, where normally there is no electrochemical activity, to a final potential $\left(E_{\text {end }}\right)$, where the reaction is mass transport controlled. This technique is the basis for other important analytical methods such as the cyclic voltammetry, pulse voltammetry and stripping voltammetry $[11,12]$. 


\subsubsection{Cyclic voltammetry}

Cyclic voltammetry (CV) is an adaptation of the LSV, by cycling the potential between two vertices ( $E_{v t x l}$ and $E_{v t x 2}$ ), originating a triangular potential waveform (Figure 3.3. A) that result in the cyclic voltammograms (Figure 3.3. B). Depending on the information required, single or multiple scans can be performed during the experiments.
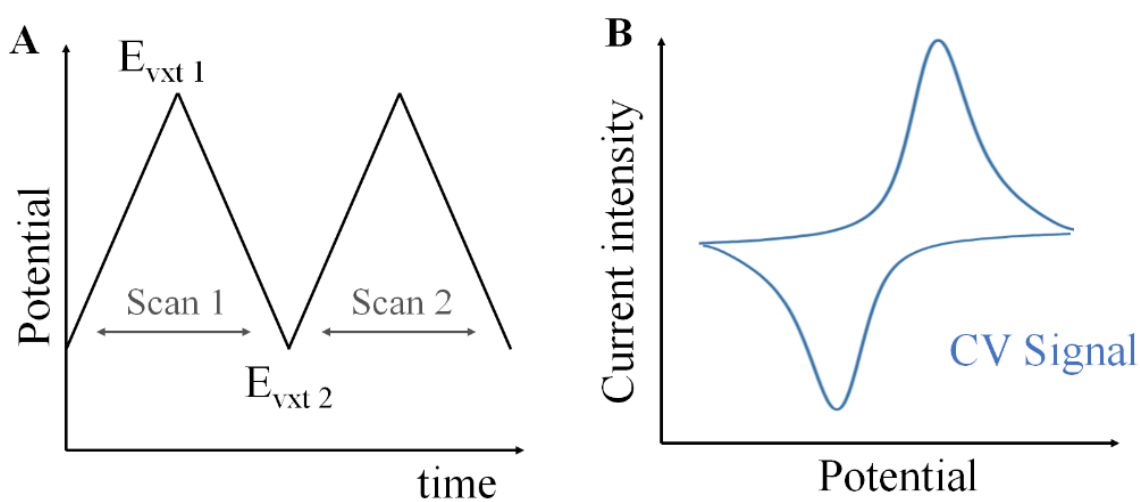

Figure 3.3. Cyclic voltammetric technique. (A) Waveform for potential cycling applied between $E_{v t x l}$ and $E_{v t x z}$ during the scans. (B) Typical cyclic voltammogram for a reversible redox process.

The fast access to considerable information on redox processes and kinetics of heterogenous electron transfer reaction, leads the $\mathrm{CV}$ to be the first experiment performed in an electroanalytical study [2,10-12,21]. The potential cycling, give information on the coupled redox reactions reversibility, indicating if a system is based in reversible, quasireversible or in irreversible processes [22]. The $i_{p}$ anodic and $i_{p}$ cathodic values, should be identical for a simple reversible couple, indicating a fast process. However, the ratio between those values can be influenced by the reactions coupled to the electrode process. Additional information can be extracted from peaks potential $\left(E_{p}\right)$, which is indicative of the formal potential of the redox process [21]. Analysis of the voltammetric peak-to-peak separation, is indicative of process reversibility. For a reversible process the peak-to-peak separation is $\sim 57 \mathrm{mV}$ and is independent of scan rate. On the other hand, in the 
electrochemical quasi-reversible or irreversible processes [2,21], the peak-to-peak separation is larger and is dependent of the scan rate for potential sweep [22]. Moreover, in this case the individual peaks are reduced in size and widely separated. Totally irreversible systems are characterized by a shift of the $E_{p}$ with the scan rate, which is caused by a slow electron exchange between the electroactive species and the WE [21].

\subsubsection{Pulse voltammetric techniques}

Introduced by Barker and Jenkin, pulse techniques were designed to lowering the detection limits of voltammetric measurements, achieving quantifications lower than $10^{-8} \mathrm{M}$ [2]. These techniques, allows to increase the ratio between the faradaic and nonfaradaic currents, since the differential measurement of the current intensities, hinder the background signal [11,18]. Quite diverse and complex schemes have been devised for applying potential steps and sampling currents, originating different pulse techniques such as the normal pulse, square wave and differential pulse voltammetry (DPV), among others $[10,12]$.
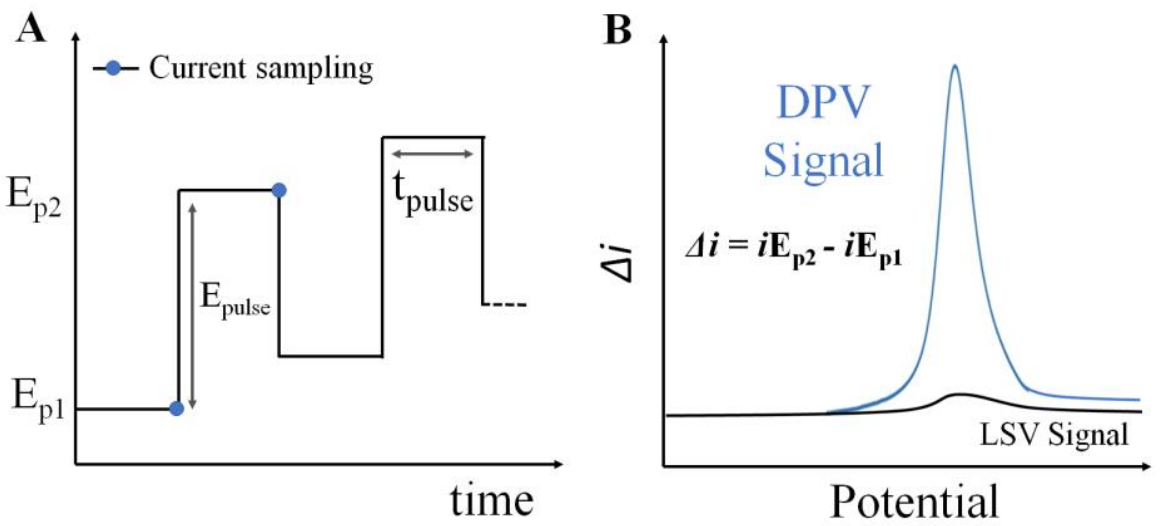

Figure 3.4. Differential pulse voltammetry. (A) Potential waveform of pulses superimposed on staircase, and (B) Typical DPV voltammogram for an interest analyte oxidation. 
The DPV, is particularly important to perform the determination of organic or inorganic trace concentrations, resulting in voltammetric peaks height directly proportional to the concentration of the corresponding analytes [2,12]. This technique uses superimposed pulses of a fixed magnitude on a potential ramp. The difference between the currents sampled before the pulse application and again later in the pulse life, generate highly sensitive voltammetric peaks, from a small-amplitude scheme superimposed on a staircase potential ramp (Figure 3.4) [10,11,23].

\subsubsection{Stripping voltammetric methods}

Stripping methods, extensively used in quantitative determination and speciation of metals, gather a variety of electrochemical procedures that include a stirring preconcentration step, usually followed by a rest period, that precedes a potential sweep step (most frequently LSV or DPV), where the pre-concentrated species are redissolved from the electrode surface (Figure 3.5) [2,10-12].
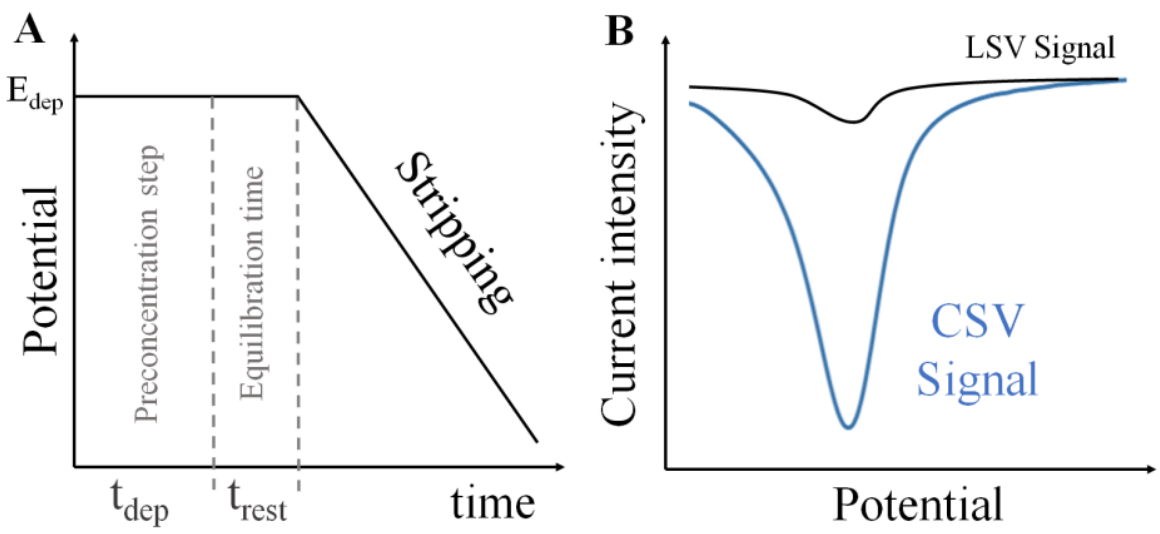

Figure 3.5. Stripping voltammetric technique. (A) Potential waveform for a typical cathodic stripping experiment and (B) Typical cathodic stripping voltammogram for an interest analyte. 
This remarkably sensitive method, can use adsorptive, anodic or cathodic modes, depending on the nature of the analyte. The first one is based on the adsorptive behavior of some analytes. During the preconcentration step, which can occur under a fixed potential or at open circuit, the adsorption of the target species onto the WE surface is promoted by the hydrodynamic conditions of the supporting electrolyte. After that, the imposed potential ramp leads to the redox reaction of the adsorbed species, which are finally redissolved.

Differently, anodic and cathodic stripping require the application of a potential during the preconcentration step. In anodic stripping voltammetry (ASV), the WE behaves as a cathode during the preconcentration step, and as an anode during the stripping step, with the analyte being newly oxidized. An opposite behavior is found for the cathodic stripping voltammetry (CSV), since the WE behaves as an anode during the deposition step oxidizing the interest analyte, which is reduced in the subsequent cathodic potential sweep [23].

The major advantage of stripping methods is related with the preconcentration of the interest analytes on the electrode surface. The species are deposited into a much smaller volume than the bulk solution one, being concentrated by factors of 100 to more than 1000 in the deposition step carried out under stirring; therefore, the signal is less perturbed by charging or residual impurity currents [10].

\subsection{ELECTROCHEMICAL PLATFORMS SETUP}

As previously mentioned in section 3.2, the electrochemical platforms are usually constituted by three electrodes (WE, CE and RE), whose are part of electrochemical cell. To perform electrochemical experiments, it is also required an ionic substance, typically a salt in solution, the supporting electrolyte, which function is to ensure conductivity [12]. Besides that, electrochemical experiments require a potentiostat/galvanostat and the corresponding software as basic instrumentation $[2,10,12,18]$. 
Several types of possible setup and apparatus are available to perform electrochemical experiments $[2,10,12,18]$. The most well-known configuration has been based in the use of the conventional three-electrode cell. However the demand of the analytical field, forced this configuration to reduce the size, increase the portability and integrate all the parts in a single device, resulting in screen-printed electrodes (SPEs) based systems.

\subsubsection{Conventional three-electrode system}

A three-electrode cell configuration usually consists in a beaker of $5-50 \mathrm{~mL}$ volume, containing the three electrodes immersed in supporting electrolyte. More complete systems that integrate gas control, for example to oxygen removal, temperature control and magnetic stirrer along with proper cover, can be also found (Figure 3.6) $[2,10]$. These systems are highly adaptable, allowing an easy alteration of the cell components, depending on the analytical purpose and/or the nature of the sample [2,10-12,18].

Frequently used as cell material, glass offers low-cost, transparency, chemical inertness, and impermeability; however, Teflon and quartz represent other possible materials [2,11].

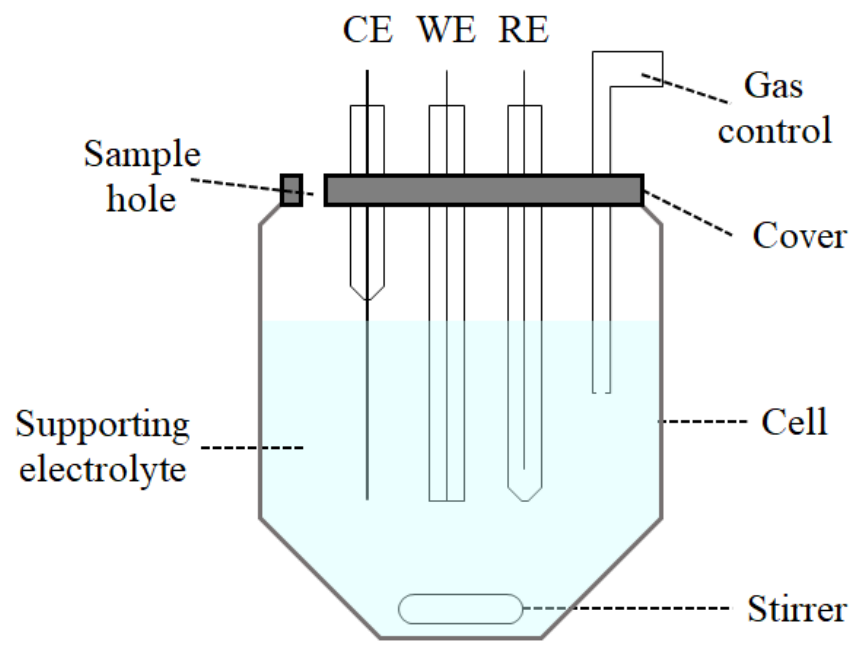

Figure 3.6. Schematic conventional electrochemical cell. The electrodes are inserted through holes in the cell cover. 
$\mathrm{RE}$ is of great importance as electrochemical cell constituent, providing the stable potential, against which the potential of the WE is compared. Usually presenting a constant composition of both forms of a redox couple, $\mathrm{RE}$ are usually made of $\mathrm{Ag} / \mathrm{AgCl}$ (silversilver chloride) or the pair $\mathrm{Hg} / \mathrm{Hg}_{2} \mathrm{Cl}_{2}$ (saturated calomel electrode). These redox pairs allows the RE to act as "buffer" against potential changes. To minimized possible contamination from RE, a platinum wire or graphite rod can be used as $\mathrm{CE}$, functioning as an inert intermediate bridge for the current flow until WE $[2,10-12,18]$.

The WE is the component that defines the performance of the electrochemical experiments, since it should provide a reproducible response with a high signal-to-noise characteristics. To select the appropriate WE, should be considered the redox behavior of the target species, which define the potential window required and the background currents of supporting electrolyte. Also, electrical conductivity, geometry, mechanical properties, cost and toxicity, are important when selecting this component. Several WE configurations, such as planar disks, rotating disk, thin-layer (FIA system), among others, enable the achievement of very distinctive electrochemical properties [2,10-12]. Numerous materials have been used as WE in electroanalytical purposes, within the most popular are; mercury, carbon, boron-doped diamond films, or metals such platinum, silver and gold $[2,10]$.

Mercury drop used as WE offers a homogeneous surface, that exhibit very reproducible and extremely sensitive electrochemical responses. This system has been widely employed for several determinations, however, the complex mounting scheme required and the extremely toxic properties of this material, led to its elimination from electroanalysis. Concerning that, solid electrodes have attracted considerable analytical interest, allowing to work in wide potential ranges and also presenting fast electron transfer rates. Nevertheless, the electrochemical response obtained with these electrodes, shows to be dependent of the surface state. These solid WEs, usually present a heterogeneous surface that require pretreatments, which are accompanied of physical and/or electrochemical polishing steps to achieve an appropriate reproducibility. The requirement of electrode pretreatment, comes from the "memory effect" as consequence of the origin and history of the surface $[2,10-12,18]$. Additional activation steps, such as chemical, heat, or laser 
treatments, have also been used to enhance the analytical performance of these probes $[2,12]$. Moreover, despite the adaptability offered and the relative low-cost of these platforms, they still present a complex mounting scheme that trouble the in situ analysis, which means, difficulties to work out of the laboratory.

In that regard, the use of SPEs, allows to overcome some of the problematic found for these conventional setup [2,24,25].

\subsubsection{Screen-printed electrodes system}

The electroanalytical restrictions and practical difficulties associated with the conventional solid electrodes, resulted in an emerging field of small-sized, disposable, sensitive and selective devices, the SPEs [8,25-27]. Indeed, the use of these transducers for electrochemical sensors and biosensors fabrication is currently widespread in electroanalysis, evidencing its suitability for various applications [2].

Produced by thick-film technology, the SPEs fabrication consists on the sequential deposition of conductive and nonconductive layers onto an insulating support (substrate for printing), which is carried out using the adequate screen-printing machines $[8,25,26]$. This technology permits a large scale production of low-cost and yet highly reproducible devices [22], being especially useful in fabrication of multiple electrodes or electrode array structures [12].

The deposition of different inks or pastes, is carried out on the substrate for printing, using the appropriate screen that holds open-mesh areas. The possibility of use different screens and inks give rise to different types of SPEs, that can be fashioned according to the analytical purpose. As shown in figure 3.7, the printing of SPEs is conducted by a squeegee that forces the ink to pass through the open-mesh areas, depositing the pattern onto the substrate for printing surface. The final devices are obtained by applying curing steps of adequate temperatures, between the printing of each pattern. Thus, the substrate retains relatively resistant ink layers, of 20 to $100 \mu \mathrm{m}$ of thickness [27]. 

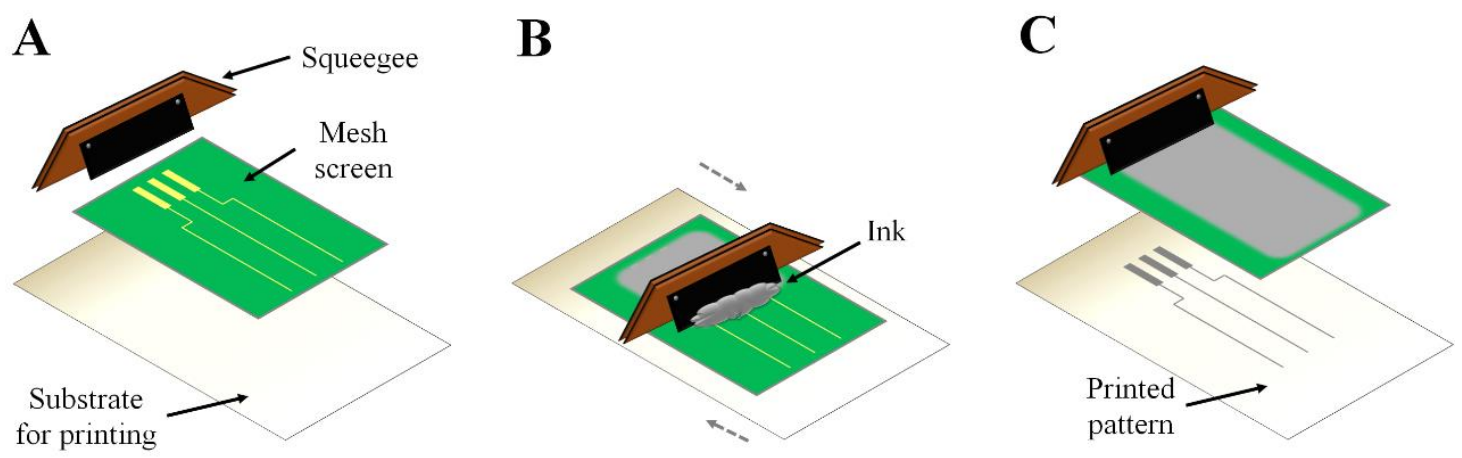

Figure 3.7. Screen-printing procedure. (A) Components for SPE fabrication, squeegee, porous screen and substrate; (B) Screen-printing of ink by squeegee traversing; and $(C)$ Ink deposited as SPE paths pattern on the substrate surface (Adapted from [28]).

Over the years, the increased reputation of the SPEs as efficient electroanalytical tools, resulted in numerous commercially available electrodes of distinct materials and configurations. In figure 3.8, is shown the typical SPEs configuration produced in our laboratories, and therefore, the ones used in this work. These home-made SPEs hold a threeelectrode arrangement, presenting a pseudo-reference electrode of $\mathrm{Ag} / \mathrm{AgCl}$ based ink (SPE $\mathrm{Ag} / \mathrm{AgCl})$, a CE usually printed with a carbon paste ink, and finally a WE of $0.126 \mathrm{~cm}^{2}$ of area, that can be constituted of carbon, platinum, gold or silver, among others types of conductive inks [25]. Finally, the last insulating layer of a nonconductive ink defines the final SPEs, whose are combined with a potentiostat through the conductive tracks and connectors made of a silver-based ink.

Besides the design adaptability, the setup of the SPEs add other exceptional advantages on electroanalytical field. SPEs are inexpensive and can be used as either disposable, or sometimes as reusable devices. Furthermore, without the requirement for highly skilled personnel and without the need of tedious sample pretreatment steps, these platforms are easy to operate and suitable for in situ and real-time applications out of the laboratory. Additionally, the low power requirement, the quick response, the high sensitivity and the 
ability to operate at room temperature, increase the range of applications for these devices $[8,22,25,29,30]$.

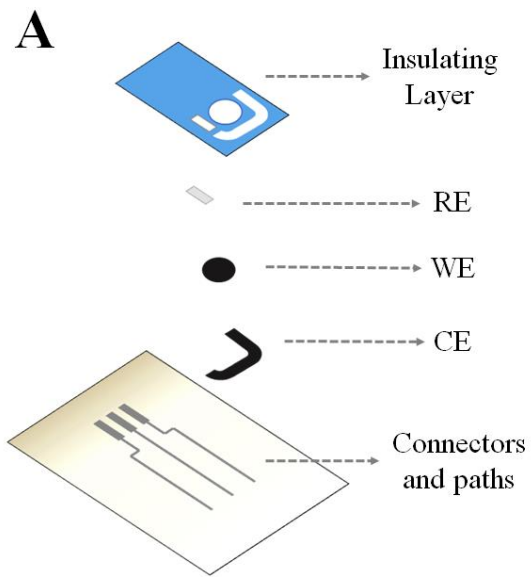

B

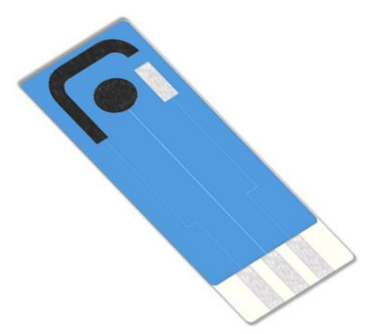

Figure 3.8. SPEs home-made at Burgos University. (A) Screen-printed layers of SPEs components, and (B) Configuration home-made SPE used in this PhD thesis (WE area $\sim 0.126 \mathrm{~cm}^{2}$ ).

Since 1990, these microelectronics adapted devices are continuously improving, resulting in the fabrication of robust and miniaturized sensors [8,30]. Advances facing the traditional methods, mainly due to the possibility of sample direct analyses in its environment, without alteration of the natural conditions, can be achieved [26]. Therefore, reinforcing the use of SPEs as a more effective analytical tools, for real-life applications of the industrial, clinical and environmental fields. Moreover, the possibility to operate with very small volumes, increase the sample throughput, allowing to employ it in parallel techniques [31].

During the last decade, the most remarkable improvement achieved in the construction of SPEs, is related with the application of the techniques in flexible substrates for printing, emerging in portable configurations for on-site testing. Many examples of flexible SPEs based on materials such as paper [32], variety of polymers [33-38], temporary tattoos [39$42]$ and textiles $[13,14,43-45]$, have been applied to an extensive range of determinations, most of them, aiming wearable purposes for bodily fluids analysis [46-48]. 
SPEs-based electroanalytical devices, allows the translating of laboratory-based studies into industry for "in-the-field" experimentation [22]. Moreover, the appropriate modification of the electrode components, offers the opportunity of simplifying the analyses of complex matrices outside of the laboratory [24]. Thanks to the fast development of nanotechnology, biotechnology and chemistry, the electrochemical and analytical characteristics of SPE-based sensors has been highly improved [1,2,4,7,24,26,49]. In the following two sections are described the types of modifications performed on SPEs, which aims to improve the devices' specificity and sensitivity towards the interest analytes [30].

\subsection{NON-ENZYMATIC SPES MODIFICATIONS}

SPEs fabrication, enable the manipulation of the ink's composition, integrating modifications that improve the analytical performance of these devices. Several electrochemical mediators [50,51] or enzymes [52,53] can be mixed with the conductive inks and printed as WEs, allowing an easy modification of the transducer's sensing properties. However, the most common modifications are performed by depositing different types of substances on the WE surface $[3,8,25,54]$.

Several types of nanomaterials have attracted the focus of electrode modifications, promoting the amplification of electrochemical signal, mainly through the increase of the surface area [54,55]. Also, metal coatings, polymers, enzymes, reactive groups, among others, have been recognized to mark the analytical performance of the devices. Currently, various investigations give rise to a great number of electrode modifications, and combinations among themselves; aiming to achieve a suitable selectivity and sensitivity $[3,8,25,54]$. Herein, electrodes modifications are briefly described and assembled according with each material used. 


\subsubsection{Carbon-based nanomaterials}

Extensively used in electrochemistry, carbon is recognized to own unique properties like wide potential window, chemically inert and suitability for different type of analysis [56]. The advances on the nanosizing of carbon, has produced high surface-to-volume ratio, high electrical conductivity, chemical stability, biocompatibility and robust mechanical strength materials, which are prone to the used in electrochemical sensing of various compounds [57]. Superior analytical performance for several analytes was achieved using electrodes modified with graphene [58-61], carbon nanotubes [55,62-68], diamond nanoparticles [69] and carbon nanofibers [70].

\subsubsection{Metallic nanoparticles and coatings}

Likewise carbon, several metals have been applied for SPEs' modifications, among them, platinum [71-73], gold [74-76] or silver [77,78] are the best known.

Metallic nanoparticles (M-Nps), especially the noble metals ones, aroused great interests due to their attractive stability, extraordinary conductivity, magnetic and optical properties. Also, their remarkable ability to catalyze electrochemical reactions, the large surface-tovolume ratio and the biocompatible properties, have been extensively used in developing novel electrochemical sensing platforms, improving their performances [25,54,79-82]. Furthermore, the simple and straightforward electrodeposition of M-Nps, by using a metalsalt dissolution and its easy nucleation onto WEs surface, result in effortless manner to obtain highly sensitive analytical signals. The use of these metals can be also extended as metallic coating, offering an alternative to the high toxicity of mercury coating at the SPEs [25]. M-Nps, also play an important role in electrochemical biosensors' development, providing a controlled microenvironment for biological molecules, retaining their activity and facilitate the electron transfer between the redox center of the biomolecules and the WE surface [79]. 
Gold nanoparticles (Au-Nps), recognized as biocompatible with low cytotoxicity, and as chemically stable material, opened the possibility for the miniaturization of sensing devices to the nanoscale, and therefore, offering excellent prospects for chemical and biological sensing $[54,79,80,82]$. Several applications for Au-Nps, such as the determination of heavy metals $[13,83]$ and anions $[84,85]$, antibody labelling for electrochemical immunosensors [86], electron-transfer between bioreceptor and WE surfaces [87] and for enzyme stabilization [88-90], can be found in sensing approaches.

Platinum nanoparticles (Pt-Nps), take advantage of both, the dimensional and functional characteristics of their surfaces, being present in extensive electrochemical applications. This noble metal nanoparticles, are highly catalytic and contains inorganic cores, which translates to specific physical characteristics, such their size and shape dependent optoelectronic properties [54,91]. Despite its high cost, Pt presents high stability and electrical conductivity, which makes this material attractive for the development of sensors. Numerous sensing applications of Pt-Nps can be found for heavy metals [92,93], antibiotics [94,95], pharmaceutical molecules [96], tumors markers determinations [97]; and also, for chlorine gas monitoring by using Pt ink as WE of the SPEs [98].

Furthermore, with significant importance in biosensing development, Pt-Nps present an catalytic adsorption-controlled mechanism for hydrogen peroxide [99]. This fact, make the use of these nanoparticles especially interesting for oxidase enzymes based-biosensors, resulting in higher sensitivities for the target analytes [64,68,100-103].

Silver nanoparticles (Ag-Nps), have proven to be one of the most important groups of nanomaterials for sensing approaches, resulting in highly sensitive sensors. Particularly important for halide anions $[44,104]$ and hydrogen peroxide $[105,106]$ determinations, $\mathrm{Ag}$ Nps, also offers the possibility to perform simultaneous determinations of dopamine and ascorbic acid [107], and also, of hydroquinone and catechol [108].

Rhodium nanoparticles (Rh-Nps), are among the less represented noble M-Nps in electrochemistry, possibly due to its high cost. However, during the last decades, their potential use as catalyst and its interesting magnetic properties [109], leads to an increase 
number of sensing strategies based in Rh-NPs. The use of this metal for biosensors construction [87,110-113], hydrazine determination [114] and to detect hydrogen peroxide $[115,116]$, can be found as example of its potential in the electroanalysis field.

Additional to these, a great variety of other M-Nps such as palladium [110,117], ruthenium [118], or even alloys with lead [119], can be also found as suitable nanostructures that improve the electrochemical response of some devices.

\subsubsection{Metallic-organic frameworks - MOFs}

Over the past two decades, an increased number of studies about metal-organic frameworks (MOFs) have been reported and recognized to possess relevant properties for sensing applications [120-124]. The use of MOFs have been applied to numerous areas of study, like gas storage and separation, as conductive or magnetic materials, for catalysis, biomedical imaging, clean energy and also for drug delivery [124-126].

MOFs are originated by linking a metallic center and an organic ligand through a strong bond, forming a low-density network with high thermal and chemical stability, tunable pore structure and dimension, and also, adjustable internal surface properties. The vast possible combinations of constituents, geometry, size and functionality originated more than 20,000 different MOFs, with extremely high porosity that originates surface areas up to $7000 \mathrm{~m}^{2} / \mathrm{g}$ [120-126].

In the past, MOFs showed electrical insulator properties, however, in last years they had demonstrate conductivity and charge mobility, an important feature for electrochemical purposes [122]. In fact, these frameworks are used to enhance sensitivity and selectivity by various charge transfers processes, occurring among different ligands and metal centers, as well as, from MOF skeletons to guest species [123]. Moreover, MOFs porosity offer a controlled nano environment, allowing the encapsulation of several guest molecules/ions into the frameworks, concentrating analytes and enhancing its detection. Also, they can contain specific functional sites, that can favor certain type of reactions [120,122-124]. 
Numerous sensing and biosensing strategies are based in these materials, offering improved sensitivity and detection limits. Peroxidase-like activity [127], preconcentration agent [128], enzymatic immobilization support [129], catalyst [130,131] or even to increase the conductivity and stability of biosensors [132], are some of the examples that can be found for the role of MOFs in electrochemical platforms.

\subsubsection{Polymers}

Polymers already proved the important role they had in the development of new sensing approaches. From pseudo-natural polymers such as chitosan (CS) [133], passing through conductive polymers [134], and going to the ultimate novelty of the imprinted polymers $[135,136]$, this type of compounds is usually used as a functional layer that improve the analytical performance of several sensors.

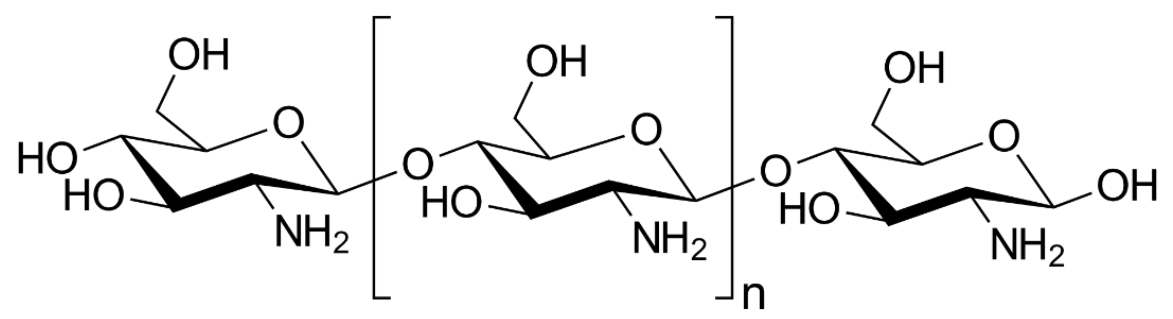

Figure 3.9. Chemical structure of chitosan (poly(D-glucosamine) repeat units.

With special advantages regarding its cationic character and biocompatibility properties [137-139], CS is a polysaccharide that displays a high content of hydroxyl and amino groups along its chains (Figure 3.9). This N-deacetylated derivative of chitin, possesses an excellent membrane-forming ability, ion transport and ion-exchange properties, high permeability toward water, good adhesion, nontoxicity and high mechanical strength. Additionally, the susceptibility to chemical modifications, allows the coupling of other 
molecules to the polymer structure, improving its electrochemical characteristics [137139].

With great interest in sensing field, CS has prompted the extensive applications as a matrix for enzyme immobilization [140-144] and other biomolecules [145], providing an excellent biocompatible microenvironment, that outcome in higher sensitivity and stability of the devices. Moreover, have also been performed using this polymer, the determination of anions $[84,146]$ and, the stabilization of the electrode components, since CS avoid the washing of such components and/or modifications from the WE surfaces $[40,147]$.

\subsection{ENZYME BASED - SPES BIOSENSOR SYSTEMS}

The use of SPEs modifications is an attractive alternative to improve the performance of these devices. Nevertheless, the great interest relies in biomodified electrodes, which achieves higher specificity for the determination of target analytes in complex matrices $[8,25]$.

The biorecognition elements, are the major responsible for the selective recognizing of the analytes in a biosensing analysis [9]. Gathering the properties of the distinct bioreceptors with the SPEs advantages, a suitable method for mass production of several low-cost biosensor configurations, can be attained $[5,8,148]$. Depending on the biochemical reaction measured, as a result of the interaction between the analyte and the immobilized bioreceptor, biocatalytic devices (using immobilized enzymes, cells, tissues and cellular organelles) or affinity devices (based on antibodies, aptamers, membrane receptors, nucleic acids), can be constructed $[2,8,149]$. 


\subsubsection{Enzymes as biorecognition elements in biosensing strategies}

Enzymes are the major biological elements employed as bioreceptors on biosensors' design, giving place to different measurable responses [5,148]. Enzymes are proteins that acts like biological catalysts, increasing the rate of the reactions without being consumed. Under physiological environment, the catalytic enzymatic reaction occurs by converting the specific substrates into reaction products, giving place to the simplified mechanism shown in equation $3.4[8,9]$,

$$
E+S \leftrightarrow E S \rightarrow E+P
$$

where, $\mathrm{E}$ is the enzyme, $\mathrm{S}$ the substrate(s), ES the enzyme-substrate(s) complex and $\mathrm{P}$ the reaction product(s).

In 1962, Clark's pioneering work showed the suitability of the use of these biomolecules in analytical purposes. Entrapping the glucose oxidase between to membranes, Clark designed the first approach for a glucose biosensor [150]. The central requisite for any biosensor construction, is that the selected enzyme must present catalytic functions for the target analyte conversion [149]. Moreover, controlled temperature, medium $\mathrm{pH}$ and ionic strength, and the presence of cofactors (a non-protein chemical product, such as NAD + or oxygen), are sometimes important and mandatory for the enzymatic activity regulation and posterior catalytic conversion of the substrates. There are different type of enzymes, which are classified according to the reaction type they catalyze. In biosensing applications, hydrolases that perform hydrolysis of substrates; and oxireductases, which catalyze redox reactions, are the most widely used for the construction of electrochemical biosensors $[2,8,9]$.

Potentiometry and amperometry, have been usually employed in biosensing, being the latter the preferable one. The simplicity of the amperometric techniques and methodology, allows the substrate distribution from the bulk solution to the electrode surface, where the enzyme catalyzes its specific reaction to respective products. The currents generated at a fixed potential, resulting from the electroactive properties of the generated products or consumed substrates, can be correlated with the target analyte concentration $[2,8]$. 
Between the challenges found for the amperometric enzyme-based biosensors, one of the most important is the establishment of a satisfactory electron transfer between the active site of the enzymes and the electrodic surfaces. As shown in figure 3.10, different mechanisms for electronic transfer can be exploited, defining in which biosensor generation is the device included $[2,8,25]$.

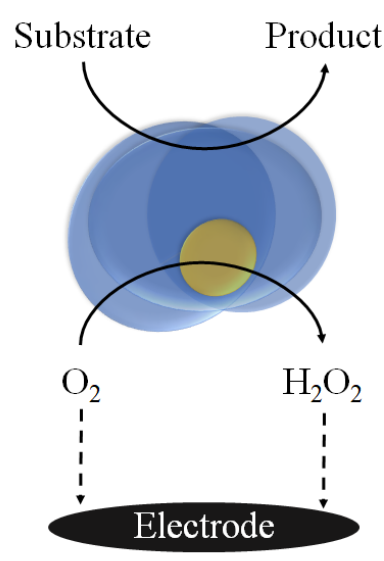

A

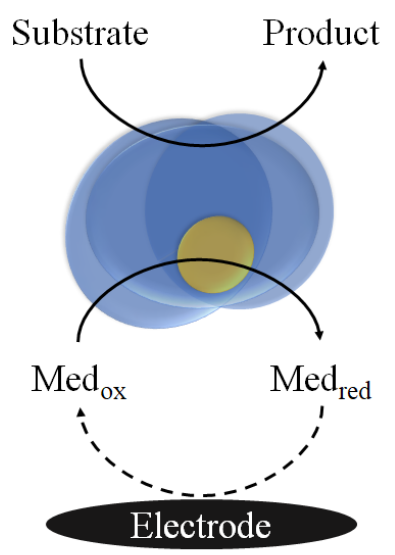

B

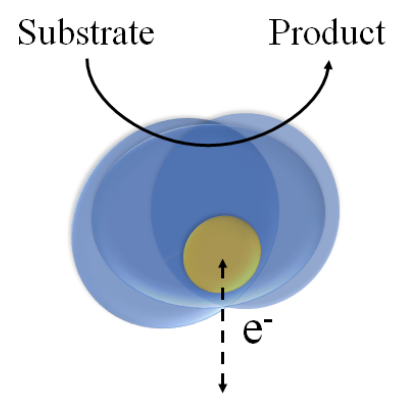

Electrode

Figure 3.10. Three generations of amperometric enzyme electrodes based on the use of natural secondary substrate. (A) first, (B) second and (C) third generation biosensors. Active center represented at yellow. (Adapted from [2]).

For the first-generation biosensor, the measurement is based on electroactive compounds, such can be the cofactors, substrates or resulting products of the catalytic reaction. This type of biosensors measure the enzymatic catalysis, monitoring the participating species of the biochemical process $[2,8]$.

Second-generation biosensors include the devices based in the use of artificial electrochemical mediators, which improve the electronic transfer between the enzyme active center and the electrode surface. These mediators can be incorporated onto the SPEs inks, facilitating the preparation of the devices. Moreover, using mediators it is possible to 
reduce the working potential required, avoiding electrochemical interferences and increasing the reversibility of electrode reactions $[2,8,25]$.

Finally, the third-generation biosensors measure the direct electron exchange between the enzyme active center and the electrode surface. The great advantage of this generation, is that the working potentials required for electrochemical response are very close to the intrinsic potentials of the enzyme itself, reducing the exposure to interferences. However, the majority of enzymes hold the active center hindered in the interior of its structure, making highly difficult the applicability of this format to several types of enzymes $[2,8,25]$.

Regardless of the biosensor generation type, the analytical response registered is an outcome of the kinetic observed for each particular enzyme. In order to evaluate the catalyst performance, several kinetic models can be applied in electrochemical biosensing strategies.

\subsubsection{Enzymatic kinetics}

Since the response of an enzymatic biosensor is highly dependent of many variables, it is important to understand the theoretical basis of enzymes' kinetic, which would help to improve the performance of the device $[2,9]$.

Using a fixed enzyme concentration, the rate of the reaction catalyzed enzymatically $(V)$, is given by the Michaelis-Menten kinetic model (equation 3.5):

$$
V=\frac{V_{\max }[S]}{\left(k_{M}+[S]\right)}
$$

where, $[S]$ is the substrate concentration, $K_{M}$ is the Michaelis-Menten constant and $V_{\max }$ is the maximum rate of the reaction. This model derives in the typical curves $V$ vs $[S]$, displaying the dependence of the reaction rate on the substrate concentration, together with the parameters $K_{M}$ and $V_{\text {max }}$. The initial rate increases linearly with [S] up to a non-limiting excess of substrate, originating the known as first-order reaction, after which, additional 
[S] causes no alteration in the reaction rate, producing a saturation zone known as zeroorder reaction $[2,9,151]$.

Regarding the kinetic curves $V$ vs $[S]$, the linear zone $\left([S]<K_{M}\right)$ is analytically useful for substrate determinations, whereas the saturation plateau $\left([S] \gg K_{M}\right)$ can be useful to determine the amount of enzyme used [2].

In the construction of biosensors, it is desirable to obtain the highest $V_{\text {max }}$ and lowest $\mathrm{K}_{\mathrm{M}}$, which the latter corresponds to the substrate concentration for which the rate is equal to half of $V_{\max }$. The $K_{M}$ value is recognized as the measure for enzyme affinity relatively to the substrate [2,9]. Based on Michaelis-Menten kinetic theory, the first-order reaction zone can be improved by manipulating the relation between the enzyme concentration and the response for substrate concentrations [2].
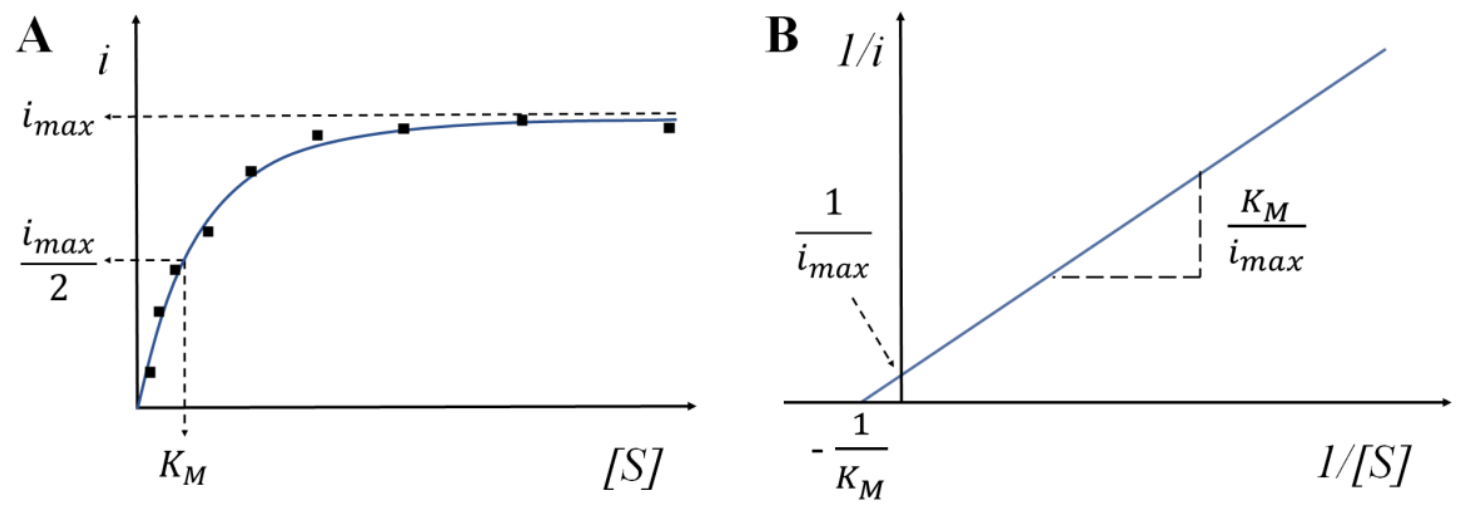

Figure 3.11. Electrochemical equivalent representations of the enzymatic kinetic based in $(A)$ Michaelis-Menten model and (B) Lineweaver-Burk plot. Typical dependence of the current registered at the biosensor on the substrate concentration.

The transference of kinetic fundamentals of the enzymatic reactions as outputs of the electrochemical biosensors, is already defined and well-known. Electrochemical equivalent Michaelis-Menten model (Figure 3.11. A), is now defined through the current generated for the substrate additions at a given time, originating the equation 3.6 [152-155]. 


$$
i=\frac{i_{\max }[S]}{\left(K_{M}+[S]\right)}
$$

where, $i$ is the steady-state current resulting from substrate additions, $i_{\max }$ is the maximum current output registered in the saturation plateau, and preserving the terms $[S]$ and $\mathrm{K}_{M}$ $[154,155]$. For the majority of biosensors, the obtained $K_{M}$ is actually a $K_{\text {Mapp }}$ (MichaelisMenten constant apparent), since the enzymes are immobilized onto the electrode surfaces. Despite that, in the representations and results presented in this thesis, the $K_{\text {Mapp }}$ is refereed as $K_{M}$. A good biosensor should present a $K_{M a p p}$ value near to the $K_{M}$ obtained for the free enzyme [151]. In the case of electrochemical biosensor devices, the rate of reaction is given in terms of current defined in amperes units [154,155].

Despite the valuable information obtained by Michaelis-Menten model, to acquire the kinetic parameters of the enzymes used in the construction of electrochemical biosensors, should be used the Lineweaver-Burk model. As shown in figure 3.11. B, Lineweaver-Burk is constructed by plotting $1 / i$ vs $1 /[S]$, giving place to a linearization of the MichaelisMenten curve and resulting in the equation 3.7.

$$
\frac{1}{i}=\frac{1}{i_{\max }}+\frac{K_{M}}{i_{\max }} \frac{1}{[S]}
$$

Since the response registered at the biosensor is highly dependent of enzymatic behavior, the biochemical processes imposed by the interest analytes in these biocatalysts' kinetics, will define the expected signal. Exists the possibility to monitor the signal obtained from the natural catalytic cycle of the enzyme, through the substrate consumption and/or products formation; or to monitor the inhibition behavior, in which the rate of biocatalytic reactions is affected [2].

\subsubsection{Catalysis-based biosensors}

Catalysis-based biosensors are the most common and widely used devices. These biosensors working mode can be evaluated kinetically, following the normal MichaelisMenten and/or Lineweaver-Burk plots, since they are constructed regarding the sensitivity 
and specificity of the enzymatic response towards the corresponding natural substrates. The amperometric responses can be registered using any type of sensor generation or redox behavior of the participant species. Several reviews can be found regarding the development of this type of electrochemical biosensors [156-159].

\subsubsection{Inhibition-based biosensors}

It is a well-known fact that some compounds such as metallic ions, pesticides, drugs and pharmaceuticals, among others; can inhibit the activity of various enzymes $[151,154,155,160-162]$. Some of those are considered toxic compounds, while others have a key role in enzymatic activity regulation of several biological pathways [160]. Regarding that the majority of these inhibitory compounds exhibit analytical interest, it is possible to develop biosensors based on the principle of inhibition [151,154,155,160-162]. In fact, several enzyme inhibition-based biosensors, have been developed, mainly by using enzymes such acetylcholinesterase [163,164], horseradish peroxidase [53,165,166], or tyrosinase [167-169].

The determination of inhibitory analytes using electrochemical biosensors, is commonly obtained from the decreased in current response associated with the inhibitor-enzyme interaction. Also, can be determined by observing the difference of the enzymatic activity when in presence and in absence of inhibitor, according to the equation 3.8.

$$
I(\%)=\frac{\left(\overline{\mathrm{A}}_{0}-A_{i}\right)}{\overline{\mathrm{A}}_{0}} \times 100
$$

where, $I(\%)$ is the percentage of inhibition, obtained through the terms $\overline{\mathrm{A}}_{0}$ and $A_{i}$, which are respectively, the intensity of the signals registered in absence and in presence of the inhibitor.

Biologically, two main inhibition types can occur due to the interaction of the inhibitors with enzymes: (i) the reversible inhibition, after which the initial activity is restored, as result of a noncovalent interaction between inhibitor and enzyme; and (ii) the irreversible 
inhibition, characterized by a covalent bonding between the enzyme and the inhibitor, with permanent loss of enzymatic activity, or a reactivation through the use of specific compounds. Moreover, as an outcome of the inhibitor binding site, different kinetic parameters are obtained, instead of $K_{M}$ a $K^{\prime}{ }_{M}$ and a $i_{\text {max }}$ ' instead $i_{\text {max }}$, indicating different types of enzymatic inhibition. Thus, it is important to identify the inhibition type, before selecting the measurement protocol $[2,9,151,160]$.

Competitive inhibition occurs when the inhibitor bound to the active center, competing with the substrates. The competition is regulated by the concentrations used in the assays, since high substrate concentrations prevents the binding of low concentrations of inhibitors. Thus, a drawback related with the obtention of low detection limits of the inhibitory analyte, is found for these systems. For that reason, is important to choose an adequate substrate concentration that compromise a good analytical signal and a satisfactory inhibition effect. As shown in figure 3.12, the concentration of a competitive inhibitor does not change $i_{\max }$ but increases the $K_{M}$ value, decreasing the affinity for substrate molecules $[160,161]$.
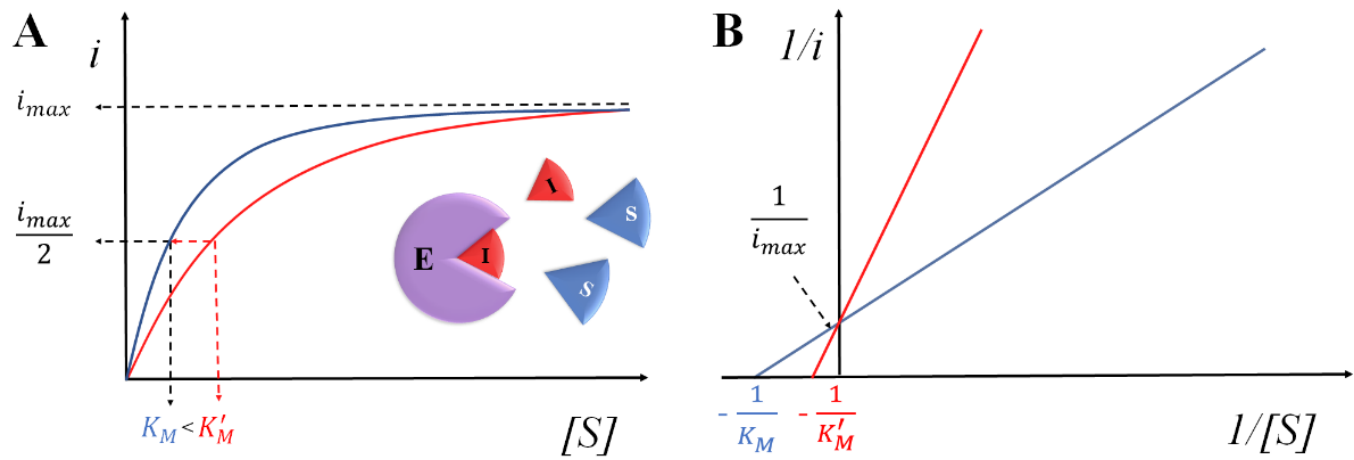

Figure 3.12. Electrochemical equivalent kinetic representation of (A) Michaelis-Menten and (B) Lineweaver-Burk plots of a competitive inhibition. Typical responses observed in absence (blue) and in presence (red) of inhibitor.

A non-competitive inhibitor is the one who bind both, the enzyme and the enzymesubstrate complex. The binding of this type of inhibitors occurs out of the enzyme active 
center, not competing directly with the substrates. In this case, the degree of inhibition is not dependent of the substrate concentration, permitting to achieve low detection limits of the inhibitory analyte.

Contrary to the competitive inhibition, a non-competitive inhibition kinetics results in an unchanged $K_{M}$, since the affinity for substrate is not affected; however, decreasing the $i_{\text {max }}$ value due to inhibitor concentrations (Figure 3.13) [160,161].
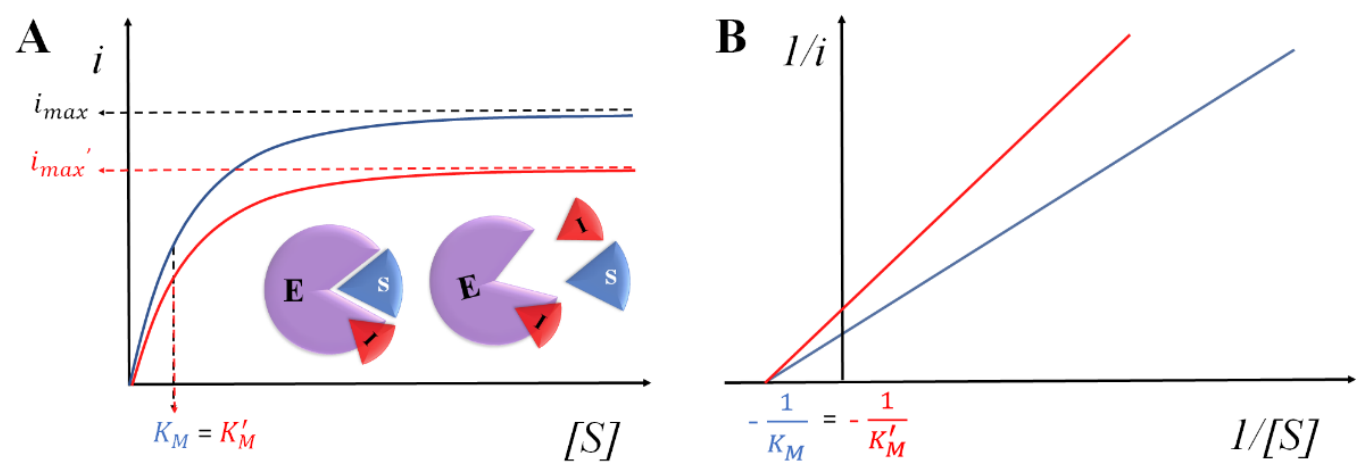

Figure 3.13. Electrochemical equivalent kinetic representation of $(A)$ Michaelis-Menten and (B) Lineweaver-Burk plots of a non-competitive inhibition. Typical responses observed in absence (blue) and in presence (red) of inhibitor.

An uncompetitive inhibitor acts in a similar way that a non-competitive inhibitor, however, it is able to bind only to the enzyme-substrate complexes. The increase of substrate concentration does not revert the inhibition degree. The kinetic of this inhibition exhibits a same factor decrease on the $i_{\max }$ and $K_{M}$ (Figure 3.14) [160,161].

Mixed inhibition is similar to the non-competitive one, however, the inhibitors binds at different sites from the active center, leading to structural changes of the enzyme, and consequently, decreasing its activity. Once again, the increase of substrate concentrations does not revert the inhibition degree. The kinetic parameter $i_{\max }$ is decrease, while the $K_{M}$ increases (Figure 3.15) [160,161]. 

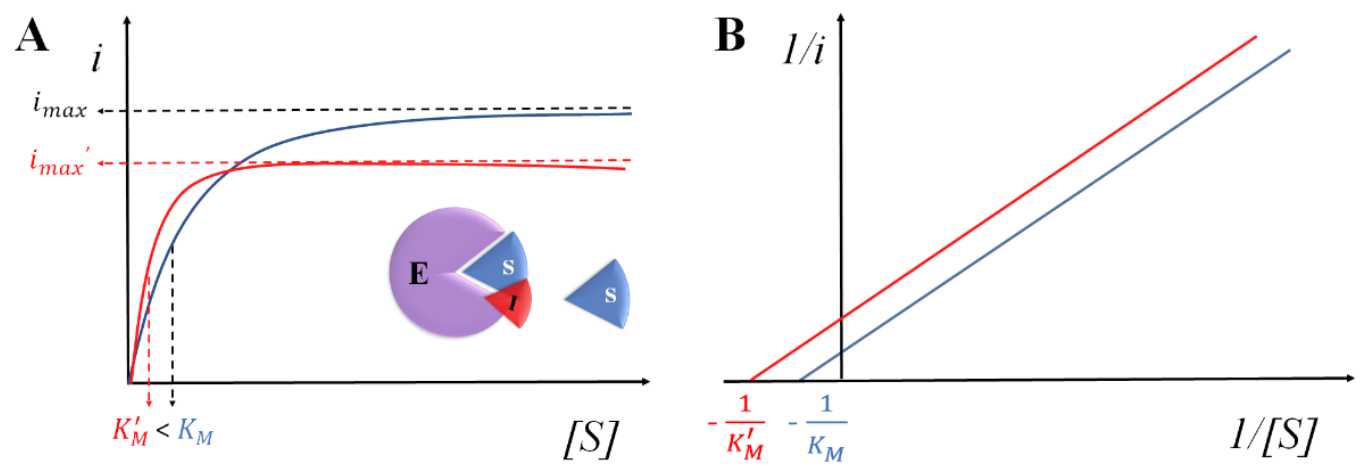

Figure 3.14. Electrochemical equivalent kinetic representation of (A) Michaelis-Menten and (B) Lineweaver-Burk plots of an uncompetitive inhibition. Typical responses observed in absence (blue) and in presence (red) of inhibitor.
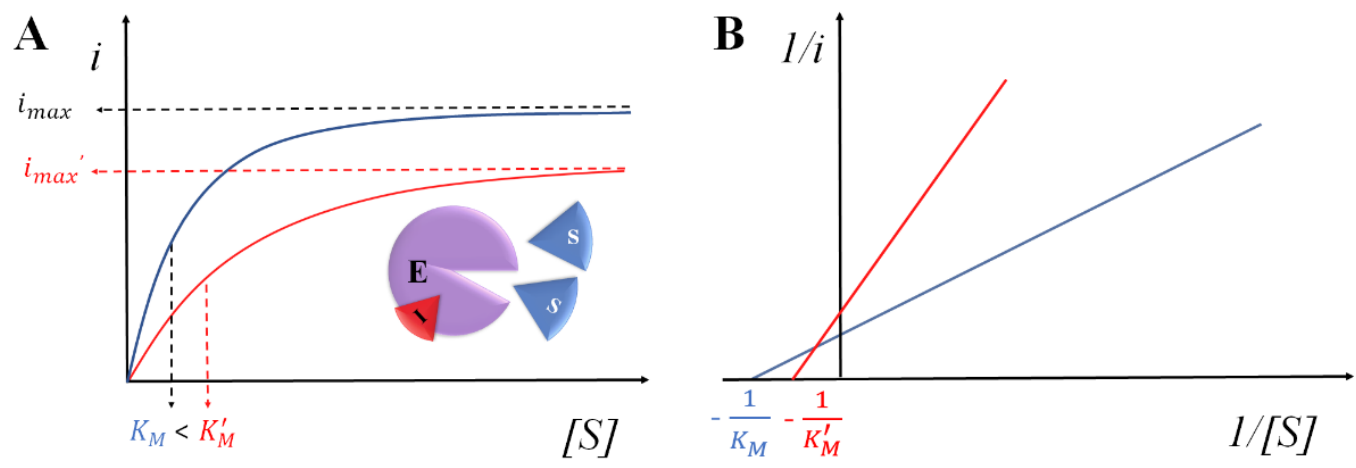

Figure 3.15. Electrochemical equivalent kinetic representation of (A) Michaelis-Menten and (B) Lineweaver-Burk plots of a mixed inhibition. Typical responses observed in absence (blue) and in presence (red) of inhibitor

An enzymatic inhibition is not always promoted by the presence of foreign inhibitory molecules. Some enzymes display substrate inhibition mechanisms, which also leads to activity loss. In these mechanisms, the kinetic curves reach a $i_{\max }$ value, after which, above a certain substrate concentration $\left(\left[S_{\max }\right]\right)$ is observed a decrease of the reaction rate (Figure 3.16 A) $[9,170]$. In this case, it is observed the formation of an inactive complex of enzyme 
with the natural substrates (ESS). Regarding this, the equation 3.4 is followed by the equation 3.9 , that reflect the impossibility to convert the substrates in the corresponding products.

$$
E+S \leftrightarrow E S S
$$

While in the ES, one molecule of substrate is in the optimal position on the active center, in the ESS, portions of distinct substrate molecules may occupy positions in the active center, avoiding the correct geometry for the reaction, and thus, prohibiting the catalytic conversion $[154,155,170]$.
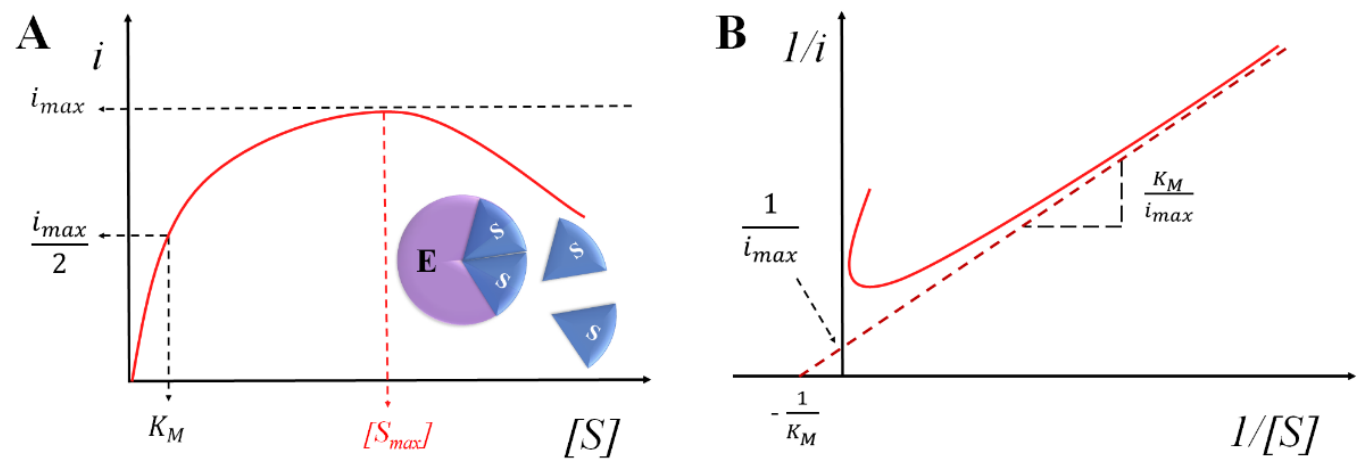

Figure 3.16. Electrochemical equivalent kinetic representation of (A) Michaelis-Menten and (B) Lineweaver-Burk plots of a substrate inhibition. Typical response observed for substrate inhibitory concentrations.

When $[S]<\left[S_{\max }\right]$, the observed kinetic is the normal catalytic one, like the described before in the section 3.6.2. However, when $[S]>\left[S_{\max }\right]$, the Lineweaver-Burk plot must be defined by equation 3.10, in which, the term $\frac{1}{i_{\max } K_{I}}[S]$ becomes significant with the increased [S]. This is noticeable in the graphic representation of Lineweaver-Burk for substrate inhibited enzymatic reactions, in which the curve $1 / i$ values are increased in near zero $1 /[S]$ values (Figure $3.16 \mathrm{~B}$ ). 


$$
\frac{1}{i}=\frac{K_{M}}{i_{\max }} \frac{1}{[S]}+\frac{1}{i_{\max }}+\frac{1}{i_{\max } K_{I}}[S]
$$

where, $K_{I}$ is the inhibition constant that measures the affinity of enzyme to the inhibitor. This $K_{I}$ value can be determined for all the previous stated types of inhibitions based in foreign molecules. However, using the substrate inhibition system, $K_{I}$ can be easily obtained from the Michaelis-Menten model, using the equation 3.11 [170].

$$
\left[S_{\max }\right]=\sqrt{K_{M} K_{I}}
$$

This type of inhibition is the less exploited for analytical purposes, yet, it seems to be suitable to perform determinations in matrices with high content of the enzymatic substrates.

Finally, it is also known that a product inhibition can occur in enzymatic systems. This behavior is observed in systems with toxic enzymatic products. The endpoint of this system occurs with the generation of inactive complexes of enzyme-product $(E P)$, which prohibit the catalytic reaction to continue [155]. In analytical point of view, this inhibition type is less important for interest analytes determination.

The knowledge about enzymatic signaling and kinetic is very important for the development of new biosensor devices. However, the correct immobilization of these biorecognition elements in the transducers, is the key for an efficient translation of information from the biochemical domain, to a chemical or physical output signal. Therefore, the critical step on construction of electrochemical biosensors is the attachment of the bioreceptor to the WE surfaces $[8,9,149,171]$.

\subsubsection{Enzyme immobilization methods}

The growing need to perform rapid and in situ analyses, resulted in development of several types of biosensors, in particular, the ones based in enzymatic responses. Different strategies have been proposed to immobilize this type of biomolecules on the WE surfaces. The confinement of enzymes on the electrode surface aims to increase of the analytical 
specificity and the stability of the device responses. Moreover, allows to improve the biosensors' usability and to reduce the fabrication costs. Nevertheless, challenges in maintain the enzymatic activity and in avoiding changes of the protein native conformation, should be exceeded without compromise the analytical performance of the devices.

Adsorption is the simplest and the first method applied for bioreceptors confinement onto electrodic surfaces. It usually consists in the deposition of enzymatic solutions onto the WE. Since this method is based on weak interactions, such as Van der Waals forces, hydrophobic and electrostatic interactions, and also in hydrogen bridges, the immobilization efficiency is highly dependent of supporting electrolyte parameters such are the ionic strength, $\mathrm{pH}$ and temperature. Considering that no chemical modifications are needed, adsorption offers a reagentless immobilization, without affecting the enzymatic activity. Nevertheless, this method display major drawbacks associated with limited mechanical stability. The adsorbed enzymes can be easily washed from the electrode surface, requiring higher number of enzymatic units to accomplish the determinations, and consequently, increasing the cost of the devices and analyses $[8,9,149]$.

Entrapment, likewise adsorption, is very simple technique from the experimental point of view. It consists of the physical retention of the enzyme in inner cavities of a porous or gel matrix such nafion, polyvinyl alcohol, hydrogels, carboxymethyl cellulose, etc. With this method, usually the bioreceptor undergoes no structural alterations; however, high diffusional barriers for the analyte towards the bioreceptor molecules are imposed, resulting in higher times of sensor responses. Occasionally, the conditions required in some polymerization processes, can result in enzymatic activity loss, affecting the devices performance $[8,9,149]$.

Microencapsulation of enzymes is based in the use of semi-permeable membranes surrounding the biomolecules which are contacting with the electrode surface. These membranes, allow the analytes and reaction products to pass through them, avoiding the bioreceptor washing. Membranes consisting in cellulose acetate, collagen and nafion, among others, can be used in this approach. The effect of the medium conditions in enzyme 
immobilization stability is negligible; however, some of the encapsulations require unfavorable conditions to form, affecting the biocatalyst activity $[8,9,149]$.

Crosslinking is the most widely used technique for enzymatic stabilization onto the electrodic surfaces [8]. Based in the creation of intermolecular bonds between the enzymatic molecules, assisted by bifunctional agents (dialdehydes, diiminoesters, diisocyanates), this method results in efficient immobilization of the biocatalysts. Glutaraldehyde (GA) is the most frequently used bifunctional agent for enzymatic crosslinking. However, this agent is recognized to present denaturation properties for protein molecules, requiring the use of lysine residues rich-proteins, such as serum albumins, to prevent enzymatic activity loss. This immobilization, can be reach in a single modification step, by depositing onto the WE surface a drop of a solution containing enzyme and crosslinking reagents $[8,9,149]$. Moreover, crosslinking usually shows good results in terms of shelf-life stability, with works describing sensors with viability of several weeks, or even months $[40,172]$.

Covalent bonding consists in the activation of chemical groups on the WE surfaces, so that they react and form covalent bonds with the nucleophilic residues of the proteins. An enzyme attachment to the electrode surfaces using covalent bonds, usually present complex procedures and require controlled conditions of the $\mathrm{pH}$ and ionic strength of the solutions. However, the minimized leakage of bound enzymes, allows the accomplishment of longterm biosensors (from 4 to 16 months), also adding the advantage of biomolecules orientation, which results in reproducible devices $[8,9,149]$. Extensively used for enzymes immobilization, self-assembled monolayers (SAM) on gold electrodes [173]; and diazonium monolayers grafting on carbon surfaces [174], are recognized as efficient immobilization carriers, but also, very effective as electron transfer between the enzyme and the electrodes.

Screen-printed enzymes is another possibility to perform the immobilization of this biomolecules on the SPEs. This method prevents the immobilization step after the SPEs fabrication, by incorporating the bioreceptor onto the working electrode inks. The enzymatic activity found for these devices is highly dependent of the inks constituents, and 
also, of the temperatures required for the ink curing process, being not applicable to all the available enzymes $[8,25]$.

\subsection{TARGET ANALYTES}

From the electroanalytical techniques and passing through the electrode setups, until its modification and biofunctionalization; the previous sections reviewed the fundamentals for the development of new electrochemical sensors and biosensors devices. Notwithstanding, the whole development is based in one question that is usually associated with a problematic: Which are the interest analytes?

An interest analyte is a molecule/compound with analytical significance, that is, the one intended to identify and/or to quantify in a given matrix [2,4,23]. Undoubtedly, the interest analytes were the driving force for the course of this work. For that reason, in the following sections are briefly summarize the importance of lactate and halide anions; and also, the recent developments carried out for its determination, highlighting the electrochemical biosensing and sensing devices.

\subsubsection{LACTATE}

With the IUPAC nomenclature of 2-Hydroxypropanoic acid $\left(\mathrm{C}_{3} \mathrm{H}_{6} \mathrm{O}_{3}\right)$ [175], and commonly known as lactic acid (Figure 3.17), this organic acid is naturally present as Land D-optical isomers. In vivo, lactic acid is dissociated to lactate and $\mathrm{H}^{+}$, and is metabolized by the enzyme lactate dehydrogenase (LDH). While some bacterial species, such as lactobaciluus spp., have the ability to metabolize both forms of lactate isomers; the mammalian cells only contain the L-LDH enzyme, which is isomer-specific for L-lactate metabolization $[176,177]$. 


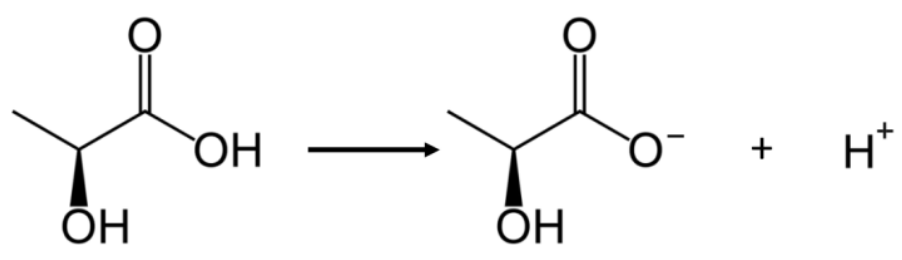

Lactic Acid Lactate

Figure 3.17. Lactic acid dissociation into lactate and protons.

Physiologically, lactate plays an important role, being fundamental when a high energetic demand exists. During the glycolytic pathway, the energy requirement results in augmented lactate production in contracting muscles. When this lactate reaches the liver, is converted back to glucose, which return via blood circulation to the muscles to produce more adenosine triphosphate (ATP), completing the Cori's Cycle (Figure 3.18) $[178,179]$.

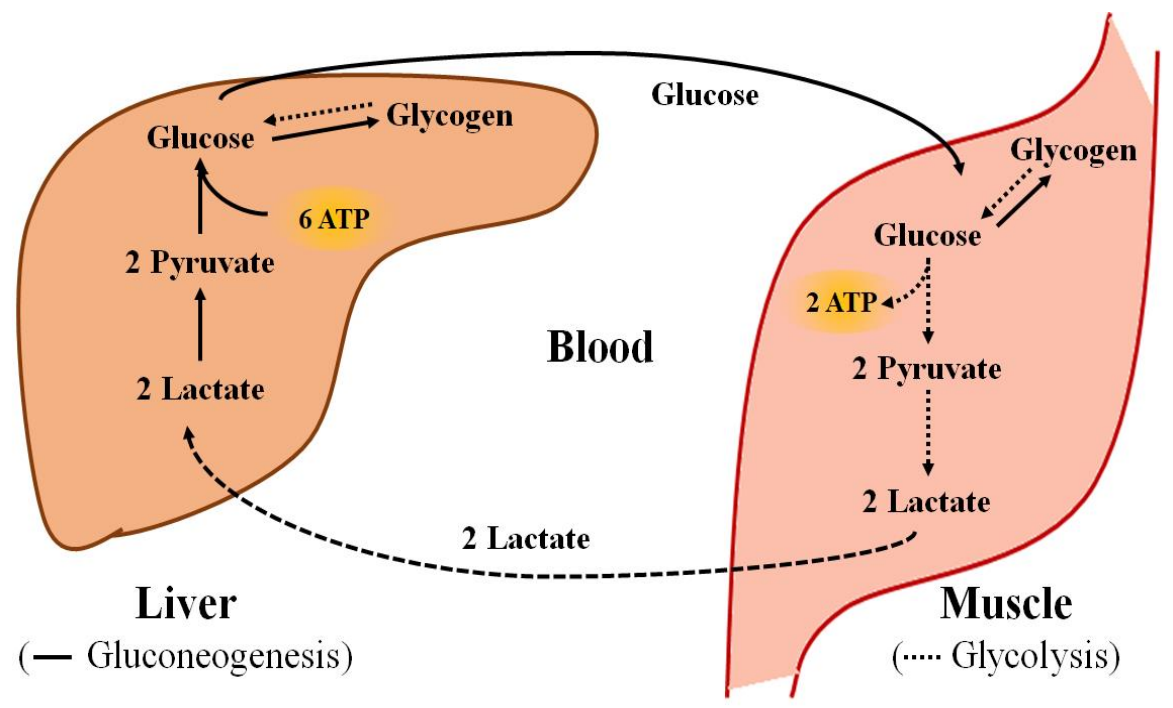

Figure 3.18. Cori's cycle - metabolic pathway of lactate production during the glycolytic process 
Despite the physiological regulation mechanisms, in some cases the organism is incapable to complete the clearance of the high lactate concentrations. The lactate physiological accumulation, is accompanied of a decreasing in tissue oxygenation, which is followed by the decrease of physiological $\mathrm{pH}$ - the lactate acidosis [178-181]. This process occurs during sports activities, with the athletes suffering muscles fatigue during intense endurance-based activities [180].

In healthy individuals, the blood lactate levels can reach $12-20 \mathrm{mM}$ concentration peaks during intense physical activity, restoring the normal levels (range from 0.5 to $2.2 \mathrm{mM}$ ) after 5-10 min [181]. However, some conditions outcome with a constant hyperlactatemia state of the individuals, being an indicative of negative prognostic in the patient status. This makes of lactate a critical metabolite biomarker for the evaluation of physical performance in sports activities, allowing to access the athletes' degree of exhaustion and for the prescription of the appropriate exercise intensities [182,183]. Furthermore, in health care applications, lactate is used to evaluate several pathologies (coronary artery disease, chronic airway obstruction, chronic renal failure, metabolic impairment) and as indicator of pressure ischemia $[184,185]$.

The significance of lactate as interest analyte is not limited to the medical and sports sectors, with an exhaustive monitoring of its concentrations in many foods and beverages. In food products, lactic acid levels are indicator for freshness, stability and quality of milk and other dairy products, as well as, in fruits, meat and vegetables. Moreover, it use is extended as food acidulant and as flavoring agent (E270) [186]. Furthermore, lactic acid is very important for wine organoleptic and sensorial properties, since it is the endpoint of the malolactic fermentation. This organic acid defines the mouthfeel, enhances the body and flavor, resulting in wines of greater palate softness and roundness. Its monitoring becomes an important quality test for wine commercialization and to preventing significant economic losses [187,188]. 


\subsubsection{Analytical determinations of lactate}

Regarding the analytical significance of lactate, a variety of analytic standard methods are available to perform the quantification in numerous matrices. One of the oldest methods is based on the colorimetric assay, in which lactate is converted to acetaldehyde in presence of sulfuric or orthophosphoric acids, reacting latter with p-hydroxyphenyl and copper ions, forming a violet-colored compound [189]. However, this method present low specificity, sensitivity and selectivity, not offering reliability of the analyses [190].

Currently, approaches based on high performance liquid chromatographic methods [191195], proton nuclear magnetic resonance spectroscopy [196-199], chemiluminescence approaches [200], fluorometric determinations [201,202] and spectrophotometric methods [203], are proposed for the analysis. In general, these methods are highly selective and sensitive, allowing to obtain reliable results; however, they require laborious steps for sample pretreatment, costly materials and equipment, skilled personnel and large times of analysis. The most important drawback of these techniques, is related with the impossibility of in situ use, prohibiting the control of lactate levels in athletes, or even, as point-of-care techniques for the monitoring of a patient status. For these reasons, during the last decade the increased number of scientific publications, patents and commercial products, shows advances in the development of lactate biosensors, as alternative analytical methods [190].

Due to the high demand for more efficient analytical tools for lactate determinations, great attention has been paid to the biosensors that use electrochemical transducers. Electrochemical lactate biosensors, most of them based in amperometric measurements, present a relatively simple and low-cost design, low detection limits, simplicity of use, sensitivity, portability, and they can be adapted to provide data remotely $[186,190,204$ 206]. 


\subsubsection{Enzymes used in lactate biosensing}

Two enzymes have been widely applied as bioreceptors in electrochemical transducers aiming the construction of lactate selective biosensors, namely, LDH and lactate oxidase $\left(\mathrm{LO}_{\mathrm{x}}\right)[186,190,204-206]$.

Strategies based in $\mathrm{LDH}$, relies on its catalytic reaction to convert lactate into pyruvate and the coenzyme $\mathrm{NAD}^{+}$to NADH (Nicotinamide adenine dinucleotides). These coenzymes, are the responsible for the electrochemical signal generation, acting as mediator to shuttle the electrons between the enzyme and electrode [204]. The oxygen is not involved in this catalysis, which make these sensors suitable for measurements in oxygen depleted surroundings [186]. Moreover, these sensors present good operational stability, long shelflife and relatively short response time [205].

Several LDH-based biosensors have been exploited [207-215]. Still, this devices require extra steps for coenzymes deposition and contention, since they present a water soluble character and are usually washed from the electrodes surface [3], therefore, complicating the in situ commitment of such platforms. Moreover, the deficient coupling of this enzyme with transducers surface, reduce the selectivity, sensitivity and dynamic range obtained with the devices [205].

The drawbacks associated with $\mathrm{LDH}$, are overcome using amperometric $\mathrm{LO}_{\mathrm{x}}$-based biosensors. Usually obtained from aerococcus viridans or from pediococcus sp., $\mathrm{LO}_{\mathrm{x}}$ (E.C.1.1.3.2.) is the preferred enzyme, due to the easy configuration of the biosensors and the simple catalytic reaction produced. This enzyme, a member of the $\alpha$-hydroxyacidoxidases flavoenzyme family, catalyze the flavin mononucleotide (FMN)-dependent oxidation of their respective substrates (Figure 3.19 A) [216-218]. The enzymatic units are two tightly packed tetramers (Figure 3.19 B), each one forming a biologically active unit. Each monomer contains $15 \alpha$-helices, resulting in $\mathrm{LO}_{\mathrm{x}}$ subunits folded in $\alpha / \beta$-barrel with two short $\beta$-strands located at the bottom of the barrel, where is located the FMN-binding site of the active center [217]. 

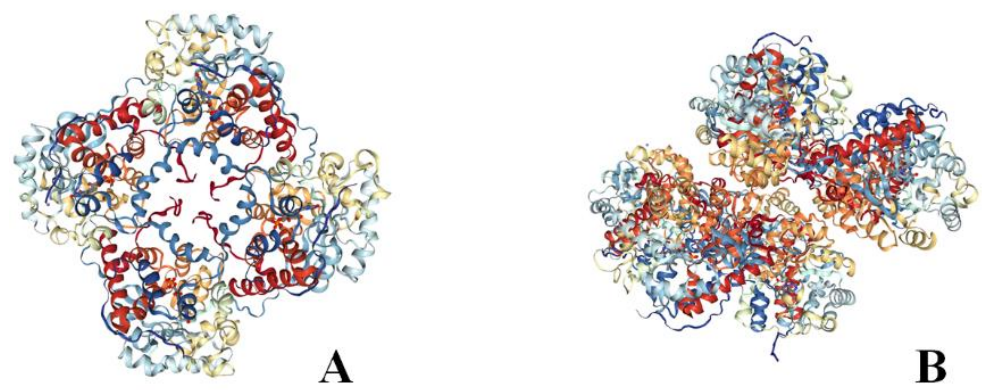

Figure 3.19. Overall structure of the Aerococcus viridans $L O_{x}$. (A)The $L O_{x}$ tetramer, describing half of the asymmetric unit, and (B) two tightly packed $L O_{x}$ tetramers. (Adapted from [217,219].)

This enzyme displays a high level of substrate specificity, discriminating lactate between other components present in problem matrices. The $\mathrm{LO}_{\mathrm{x}}$ catalysis of lactate to pyruvate, occurs in presence of oxygen, with the hydrogen peroxide $\left(\mathrm{H}_{2} \mathrm{O}_{2}\right)$ as catalytic endpoint (equation 3.12).

$$
\begin{aligned}
& \text { Lactate }+\mathrm{O}_{2} \stackrel{\mathrm{LO}_{x}}{\longrightarrow} \text { Pyruvate }+\mathrm{H}_{2} \mathrm{O}_{2} \\
& \mathrm{H}_{2} \mathrm{O}_{2} \longrightarrow \mathrm{O}_{2}+2 \mathrm{H}^{+}+2 e^{-} \\
& \mathrm{H}_{2} \mathrm{O}_{2}+2 \mathrm{H}^{+}+2 e^{-} \longrightarrow 2 \mathrm{H}_{2} \mathrm{O}
\end{aligned}
$$

The detection mechanism is usually based on oxidation of $\mathrm{H}_{2} \mathrm{O}_{2}$, by applying positive potentials that generate a current proportional to the amount of lactate; and also, restoring the former concentration of $\mathrm{O}_{2}$ (equations 3.13). Other detection strategy is based on the $\mathrm{H}_{2} \mathrm{O}_{2}$ reduction, which occurs at negative potentials (equations 3.14). Alternatively, some detection approaches are based in the use of electrochemical mediators to carry electrons between the enzyme and the electrodes [190,205]. 


\subsubsection{Electrochemical biosensors for lactate determination}

In a recent report, Kucherenko et al., reviewed the electrochemical biosensors for lactate determination. They stated the increased interest for this analyte determination over the last decades, with $31 \%$ of the total research published in last 5 years. Moreover, the stages of development of these devices exhibit important advances, with 52\% of the biosensors tested in real samples and 5\% available as commercial products [190]. The majority of these works take advantage of the use of SPEs as transducers for biosensor fabrication, determining lactate in different types of samples, using the simplicity of the amperometric technique. The following points summarize some of the electrochemical approaches proposed to perform lactate determination.

(i) Lactate biosensors in food applications: Several biosensors are described for general applications, being only tested in model samples [123,132,220-225]. However, exist a considerable number of papers concerning lactate determination and monitoring in foods, with especial interest in wines and beers [187,226-231]. Several strategies were adopted to improve the selectivity and sensitivity of the devices. Bravo and co-workers, increased the biosensor sensitivity modifying the SPCE with gold nanoparticles; however, attaining a high limit of detection [227]. Others authors applied metal oxides [222] and carbon nanotubes [226] to achieve improved performances of the devices. Other, focused the efforts in decreasing the potential required for $\mathrm{H}_{2} \mathrm{O}_{2}$ electrochemical accompanying, using bienzymatic sensors with HRP [187,231] or using electrochemical mediators [132,232]. Moreover, the use of platinum as surface for monitoring $\mathrm{LO}_{\mathrm{x}}$ catalytic reaction is frequent, allowing the development of highly sensitive devices with low working potential requirement [233].

(ii) Lactate biosensors for blood analysis: Despite the utility of lactate biosensors in food applications, the great interest is centered in health care and medical applications. Indeed, the total of the lactate biosensors commercially available, are described to perform blood lactate determinations, requiring the invasive sample extraction from the patients/athletes [234-239]. These devices are price competitive, offering low-cost analysis based in 
disposable chips. Other works approached this sample analysis [232,240], however, as the commercial ones, they only provide a single-point measurement, lacking the ability to offer continuous information on lactate level fluctuations in a period of interest [206].

In view of the wide demand for the development of noninvasive, fast, reliable and continuous measurement methods for monitoring lactate, perspiration and saliva, seems to be suitable matrices for its determination, especially for sports medicine applications, once the blood sampling can be avoided [241].

(iii) Lactate biosensors for saliva analysis: Saliva is easy to obtain and has been considered extremely attractive for noninvasive monitoring of lactate, due to its continuous and convenient availability. Lactate levels on human saliva depend on a person's metabolism and physical performance. Still, high correlations are established with their corresponding blood concentration, presenting a normal dynamic concentration range from $0.1 \mathrm{mM}$ to 0.5 $\mathrm{mM}$ of lactate [242-244]. Some reports describe higher upper limits for this range, determining lactate at $1.8 \mathrm{mM}$ [245].

The development of electrochemical biosensors to determine this analyte in saliva, is relatively recent [33,245-247]. The fabrication of SPEs integrated onto a mouthguard, for continuous monitoring of saliva lactate, was proposed by Kim and co-workers, with applicability in diverse biomedical and fitness applications. Nevertheless, the transference of the concept to a real application is not shown [33]. Other approach based in $\mathrm{LO}_{\mathrm{x}}$ immobilized onto electropolymerized phenolic films at needle platinum electrode, was developed for saliva; however, the applicability in vivo was limited, since the sterilization step was too aggressive for the electrode modifications and the device exhibit problems of permeability [246]. Claver et al., developed an approach based in electrochemiluminescence for saliva analysis, exhibiting a rapid, sensitive, but also complicated method [247].

(iv) Lactate biosensors for perspiration analysis: Sweat is also a noninvasive sample, being the most indicated matrix for lactate monitoring. Perspiration occurs naturally and is augmented in athletes, and also, in patients with certain medical conditions. One of the 
advantages for this sample, is the facilitated integration of the biosensors as wearable devices, which are suitable for on body measurements. During the last decade several papers concerning this matrix have been published [38,40-42,240,248].

One of the challenges for this sample analysis, relies in the disagreement that exits regarding the correlation with the blood levels, with contradictory results published. Despite this unclear relation, the lactate levels in sweat are known to be increased in intense exercise activities [249,250], which reflect the glandular metabolism of eccrine sweat [251]. Lactate represents $0.28 \%$ of the average constituents of the sweat organic acids, with a dynamic range usually reaching an maximal of $25 \mathrm{mM}$ [252]. However, some publications reported sweat lactate concentrations from $3.7 \mathrm{mM}$ up to $50 \mathrm{mM}[253,254]$.

Wearable setups were developed using a flexible integrated sensing array [38], a microfluidic chips [41] and a flexible patch [42]. Jia and co-workers developed the first approach of an electrochemical biosensor tattoo based in crosslinked $\mathrm{LO}_{\mathrm{x}}$. They measured the TTF (tetrathiafulvalene) electrochemical mediator oxidation, achieving good performance of the device; however, exhibiting an limited range, only up to $20 \mathrm{mM}$ [40]. The use of normal SPEs [240] and conventional electrode configurations [248], was also explored.

In overall terms, some limitations still exist and should be overcome, by developing new devices. Some of the biosensors require a great number of enzymatic units to have a suitable electrochemical performance, which results in expensive devices due to the high price of the enzymatic bioreceptor. Moreover, despite the enzymatic units used, the majority of the developed biosensors seems to be restricted in the upper limits of the lactate calibration, lacking a full range of calibration for sweat analysis. Some of the works shows promising results; however, they seem to be limited to only one type of samples, excluding the total possibilities of lactate determination and applications. 


\subsubsection{CHLORIDE}

Chloride $\left(\mathrm{Cl}^{-}\right)$is the most widespread halide anion in nature, playing an important role in many fields such as pharmaceutical, medical [255], food [256,257], environmental and agronomical [258-261].

The concentration of this inorganic anion is very useful for hydrology and geology studies, allowing the tracking and mapping of the flow of water through territories. Furthermore, chloride concentration is an indicator of soil conditions over time and growing seasons, defining the soil salinity, an important indicator for agricultural management. Such values are significant in fertilizer applications, precision irrigation and in control of environments' salinity [261].

In food-related applications, chloride has been reported to have an antimicrobial action and is commonly used as preservative, but also as seasoning in the products [256,257]. These properties are reached by adding sodium chloride (table salt) to the food; however, also adding risks for the consumers' health. The World health organization (WHO) stablished a relation between the consumption of salt and cardiovascular diseases, implementing actions to decrease the consumption of salted food products. In this context, food companies may perform a controlled monitoring of the chloride content of the products, to meet the WHO's suggestions, without compromising consumers' demands [262].

In part related with high salt content-foods; but also, extremely important in a biological point of view, chloride play an important role in human health. As the most abundant anion in the human body, chloride is the main responsible for maintaining the organism homeostasis [255,263]. The maintenance of the constant total body chloride levels, is a result of the kidneys' ability to vary the anion excretion, providing serum concentrations within a narrow range, despite marked daily variations in intake. Therefore, a deregulation in its levels, is an indicator of specific clinical disorders and conditions [263].

As a direct outcome from kidneys malfunction, a urinary chloride content out of the normal range $\left(110-250 \mathrm{mmol} \mathrm{L}^{-1}\right)$, can be an indicator of acute kidney injury. Additionally, based 
on the electroneutrality principle, urinary chloride concentration can be used to access levels of urine's major cations, such as sodium and potassium [264,265]. Moreover, conditions of hypochloremia (reduced chloride concentrations) and hyperchloremia (increased chloride concentrations), are found for chloride concentrations out of the normal

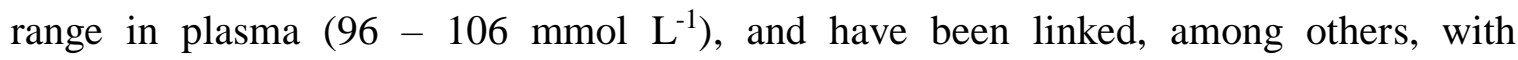
development of reduced renal blood flow, excess morbidity and mortality in critically ill patients $[263,266,267]$. Even more, this anion showed a relation with amyotrophic lateral sclerosis, since the cerebrospinal fluid from the patients, hold lower levels than the normally expected in this matrix [268].

Nevertheless, the major clinical focus for this analyte determination, has been given to the diagnosis of a genetic disease, the cystic fibrosis (CF). This progressive condition is a consequence of a gene mutation, that express a dysfunctional cystic fibrosis transmembrane conductance regulator (CFTR). The CFTR, is a chloride selective transmembrane channel that allows the movement of chloride anions to the cells surface. The mutation affects the number and/or the ability of the CFTR to transport correctly the chloride anions, creating a depletion of water on the surface of the epithelial cells, which outcomes in the presence of a thick and sticky mucus in various organs [269]. CF patients can suffer of gastrointestinal and nutritional abnormalities, salt loss syndrome (chronic metabolic alkalosis), male infertility and chronic sinopulmonary diseases. The latter is the further evident symptom, with the mucus clogging the airways and trapping germs, therefore, resulting in a persistent lung infection, chronic cough and sputum production [270,271].

Another particularity of $\mathrm{CF}$ disease is the increased chloride concentration found in patients' sweat, which make of this noninvasive matrix a preferable target for the disease diagnostic [270]. Sweat is a hypotonic biofluid produced by glands located in the epidermis and is constituted by, among others, water and electrolytes (chloride, sodium, potassium) $[253,254]$. Such substances are stored in sweat glands, secreted and finally transported to the epidermis surface. In healthy individuals a partial selective reabsorption of sodium and chloride occurs during transportation, resulting in hypotonicity of the secreted sweat. However, in CF patients the disease reflect changes in sweat chloride concentration 
[275,276]. The negative CF individuals should present sweat chloride levels below than 30 - $40 \mathrm{mmol} \mathrm{L}^{-1}$; being considered for genetic $\mathrm{CF}$ test, those individuals which levels are present at intermediate levels, between 40 to $70 \mathrm{mmol} \mathrm{L}^{-1}$. Usually, for levels higher than 70 or $80 \mathrm{mmol} \mathrm{L}^{-1}$, the disease is confirmed [270-274,277].

In 2006, the worldwide CF affected individuals was estimated to be of 60,000 , being central the development of new and efficient diagnosis tests [269]. Nowadays, genetic tests to screen the mutation and also the pilocarpine iontophoresis sweat test, are the standards for the disease diagnostic [270-274].

Also important for a different field in the medical sector, sweat chloride can be monitored in sports medicine applications. Chloride is the most abundant anion in perspiration [253], fact that make it a potential biomarker for electrolyte loss during the endurance activities [278].

\subsubsection{Analytical determinations of chloride}

The analytical interest in chloride anion has been recognized for a long time, with several methods, principles and technologies described in the literature for its quantification. However, the large range of applications, the very distinct nature and complexity of the matrices, the selectivity and sensitivity of the devices, and the economic commitment required nowadays; make of such determinations challenging in the analytical field.

The oldest approaches to perform chloride determinations, are based in volumetric techniques coupled with Mohr's [279], Volhard's [280,281] and also mercurimetrical methods [282]. These determinations are of great precision, being widely used in chemical, food and pharmaceutical industries. However, the use of very expensive reagents, the tedious and time-consuming experimental procedures, make them extremely costly and complex techniques. Moreover, the interference by other components of the sample, results in the need of exhaustive sample pretreatments to remove such substances before the measurements [257]. 
More recently, methods based in ion-chromatographic analysis [283,284], resonance light scattering [285], turbidimetry [286], colorimetry [287,288], fluorescence [289] and spectrophotometric methods [290-292], have been suggested for chloride determinations. Despite the improvement in terms of analytical performance, they still present some drawbacks regarding the cost, time of analysis, requirement of large sample volumes, as well as, the use of organic solvents, the complicated separation and extraction procedures from the samples [292].

In CF diagnosis, the pilocarpine iontophoresis (Wescor Macroduct ${ }^{\circledR}$ coil system + Sweat.Check ${ }^{\mathrm{TM}}$ ) require a sweat volume that is collected for 30 minutes, to ensure an average sweat rate. After that, the sweat need to be injected into an analyzer, in which the sample conductivity is measured and associated with sweat concentrations of sodium and chloride [293]. Despite the use of iontophoresis as standard technique, it require several steps of sample collection and analysis. Moreover, the analysis is susceptible to errors, requiring trained personnel and a careful handling of the samples. Also, this analysis is based in the sample conductivity, which can add levels of uncertainty.

\subsubsection{Electrochemical chloride sensors}

The previous stated techniques and their disadvantages calls for the search of new analytical tools, demanding simplicity, quick response and sensitive methods for the determination of chloride ions. Electrochemical platforms are adequate to achieve specific, rapid and lowcost analysis, able to be applied in several types of matrices. In addition, they are simple to use, precise and reproducible techniques.

Potentiometric approaches have been widely used for chloride determination in several matrices. They used potentiometric chloride - ion selective electrodes ( $\mathrm{Cl}$ - ISE), which are based on a chloride selective membrane, that separates the transductor and the solution, measuring the potential as function of the anion concentration [294-297]. The use of $\mathrm{Cl}-$ ISE have a lower cost per analysis, when comparing with the non-electrochemical techniques. However, they still require a considerable sample volume and pretreatment, 
and also, the associated fragility and limited life of the $\mathrm{Cl}$ - ISE, require replacement of the electrodes at a given time. These drawbacks, opened doors to the search of more efficient analytical tools, resulting in numerous works using SPEs in the recent years $[35,36,299$ $304,38,43,44,46,48,104,261,298]$.

The electrochemical sensing of chloride determinations using SPEs have shown to be based in two main strategies:

(i) The Nernstian shift of control species' voltammetric peak, which is based in the effect of chloride anions in the pseudo-reference SPE $\mathrm{Ag} / \mathrm{AgCl}$ equilibrium, outcoming in the alteration of the peak's potential. The measurement of the potential shifting of the voltammetric peaks of ferrocenemethanol (control specie), allowed the quantification of chloride content in seawater, commercial saline solution and sweat $[43,300]$. However, the need of control species add interferents to the analysis, the impossibility of continuous measurement and the requirement of sample preparation.

(ii) Other strategy is based on the formation of stable compounds between silver and chloride, which has been also extensively used for the anion determination. The excellent electrical conductivity of silver and the electro-catalytic activity for chloride anions, allows the monitoring of their reaction onto WE surfaces. The use of chemically synthetized [305] and electrodeposited [44] Ag-Nps onto the WE surfaces of the SPEs, have shown to be a proof-of-concept for chloride determination in synthetic sweat samples. Also, the use of WE of Ag ink (SPAgE) allowed to determine the anion in serum, urine, sweat and seawater [104]. Notwithstanding, silver-based sensors present problems such as their complex surface preparation, electrode fouling, impractical continuous measurement, and reduced analytic ranges.

Moreover, the amperostatic coulometry, voltammetric and potentiometric approaches, were also successfully applied to determine chloride in soils [261], brines, meat [302] and sweat [298,301] samples; however, resulting in complicated data acquisition. 
The use of SPEs allows a low-cost and on-site testing, which is not possible with other techniques. Moreover, these sensors can be printed on flexible substrates, aiming on body determinations for sweat chloride.

In view of an efficient analytical tool, the development of new, fast and simple methods of chloride determination may go through the use of Pt-based SPEs, in response to the problems associated with silver-based SPEs. Platinum (previously stated in section 3.5.2, page 37), shows to be susceptible to chloride anions, especially, when submitted to anodic potentials, discharging chlorine gas. At Pt surfaces, chloride can compete with oxygen [233,306-309], hydrogen [306,309], phosphates [233], hydrogen peroxide [233], hydrogen sulfate anions [310], perchlorate anions [310] and hydroxide anions [309], demonstrating a degree of selectivity. This selective chemisorption process is dependent of a PtO layer formed on electrode surfaces, resulting in chloro-complexes, such as $\mathrm{PtCl}_{4}{ }^{2-}$ and $\mathrm{PtCl}_{6}{ }^{2-}$ [233,306-311]. Murugappan and coworkers, take advantage of this behavior, using a Pt electrode surface for chlorine gas determination, reducing it to the corresponding anions after the gas partition into a liquid phase [98]. They achieved good results for gas detection; however, the sensor exhibit a poor analytical range and relatively high detection limit, being not able to be applied for chloride anions determination in liquid samples.

\subsubsection{BROMIDE}

Bromide $\left(\mathrm{Br}^{-}\right)$is an element present in a wide variety of matrices, such are the pesticides, medicines, biological fluids, industrial solvents, gasoline additives and environmental samples [312]. Moreover, this anion seem to be essential for biological processes, playing an important role in membranes assembly and tissue development [313].

In the $19^{\text {th }}$ century, small doses of this halide anion were first used in medicine as potassium bromide, exhibiting antiepileptic, sedative and anxiolytic properties [314-316]. However, the use of this anion have an associated toxicity, exhibiting long half-life on body (about 10-12 days). Bromide is preferably reabsorbed over chloride in kidneys, which results in a narrow therapeutic window. Known as bromism (or brominism), this toxicity outcomes in 
neurologic, psychiatric and dermatologic symptoms, accompanied of bromide increase in plasma, that can easily be confused with an hyperchloremia clinical picture. This fact makes important to obtain an accurate monitoring of bromide, in presence of chloride [317]. This problematic was the responsible for the removal of bromide salts from the most US medications in 1975, having been proved that such formulations were the responsible for $2 \%$ of the admissions to psychiatric hospitals [314,315]. Nowadays, bromide is still used as antiepileptic pharmaceutical for veterinary applications [318,319], and is also present in formulations for human medical use, which make it an important parameter to control in pharmaceutical industry [316,318-320]. Besides the control and regulations for these formulations, the easy online access to relatively unregulated medications and products is a serious problem for medical field. Romina and Stone reported a clinical case of bromide toxicity, after consumption of Dead Sea salt purchased online. Dead Sea is reported to be the body of water that contain the highest bromide concentration all over the world $(\sim 63$ $\mathrm{mM}$ ), being worrying the unregulated sell of this type of products. The symptoms of bromism are usually reversible with cessation of the anion ingestion, being very important to have an efficient diagnosis tool for an early detection of the poisoning [314,321].

The toxicity from bromide has also been reported a problem in waters, where this halide is commonly present as a natural trace element. The typical concentrations of bromide in water are approximately $0.12 \mu \mathrm{M}$ in desalinated water, $6 \mu \mathrm{M}$ in fresh water, being usually present in higher concentrations in seawater $(0.75-0.88 \mathrm{mM})$ [322]. The problematic comes from the presence of higher concentrations of the anion, that result in the formation of undesirable brominated disinfection byproducts (DBPs), during the oxidizing disinfection treatments by chlorine or ozone, used to obtain potable water [323-328]. These DBPs, are associated with adverse impacts to human health, being highly carcinogenic at very low concentrations. In addition, the presence of this anion is a potential source of bromine, which is highly reactive with natural organic matter [325,329].

Sources of bromide contamination have been associated with brines generated from oil and natural gas production. The flowback and produced waters from hydraulic fracturing of shale gas are recognized to contain $\sim 12 \mathrm{mM}$ of bromide. Additional contamination is 
originated form the use of bromide in hydrogeology, where the anion is artificially introduced as a tracer to track subsurface water flows. Wastes and unused residues result in widespread ground water contamination, that is later incorporated in potable water pretreatments $[312,323,324]$.

Moreover, this halide exhibit fireproofing properties, being used as additive in brominated flame retardants in production of electronic devices, clothing, furniture, and electrical appliances. However, the weakly bond characteristic of this additive, making it highly susceptible to be released into the environment. Nowadays, the current regulations restrict the use of organobrominated compounds, categorizing them as persistent pollutants, due to their potential bioaccumulation in ecosystems and their toxic effects on wildlife [326,330].

Therefore, analytical methodologies to monitoring of bromide anions in drinking waters, including bottled ones is an important task [325,328,331].

\subsubsection{Analytical methods for bromide determinations}

After the problematic of bromism associated with medicines, the awareness of bromide as an interest analyte, was not a major concern until the last decade. Indeed, the majority of publications regarding the halide determination appears before 90's and after 2015.

In water analysis, the standard methods for determination of bromide involve ion chromatography, capillary ion electrophoresis with indirect UV detection and UV-vis spectrophotometry [332]. An example of a spectrophotometric determination is based in the reaction of methylene blue $(\mathrm{MB})$ with bromide. In this reaction for the determination of bromide anions is based on the oxidation to bromine and the subsequent decolorization of MB, monitored at $745 \mathrm{~nm}$ [333]. However, the lack of selectivity and the different sample matrices where the determination can be relevant, resulted in the setup of alternative analytical approaches. 
Different methods based on volumetric techniques [334], spectrophotometry [325,335$338]$, colorimetry [328,339-341], ion-exchange chromatography [342,343], inductively coupled plasma mass spectrometry [344,345], spectrofluorimetry [321,331,346,347], molecular absorption spectrometry [330] and neutron activated analysis [336], have been used to determine bromide content in several types of samples. Despite the high accuracy and detection limits achieved by some of these techniques, still exists lack of selectivity, requiring complicated pretreatments and bromide extraction steps from the samples. Moreover, the high cost of the equipment, the time required in the analysis, the need of specialized personnel and the impossibility for in situ analysis make of these techniques limited to laboratory facilities.

Nowadays, the analytical bromide determination, demand for the development of simple, rapid, sensitive and portable alternatives, which would be highly desirable for in situ field monitoring of this halide [331].

\subsubsection{Electrochemical approaches for bromide determination}

The use of electrochemical approaches for bromide monitoring, seems to be underestimated, with only a few numbers of publications regarding it determination. Notwithstanding, the valuable use of electrochemistry offers the in situ possibility and a efficient low-cost analysis.

Several potentiometric approaches based in bromide ion selective electrodes (Br-ISE) were developed, and despite the fragility of the electrodes and the need for sample preparation, they proved their simplicity and selectivity for determining the halide anion [317,348-350].

One of the oldest electrochemical approaches was developed by Colovos et al. [351], that applied stripping voltammetric techniques at a mercury electrode, to determine the total halides (chloride + bromide), registering a broad peak. To separate both halide anions, the authors needed to change the anodic potential applied before the potential sweeping, in order to avoid the chloride contribution. Aside from the use of toxic mercury electrodes, 
additional drawbacks related with the presence of interferences from sulfide and iodide, are also found.

Amperometric techniques were also applied for bromide determination in bromide/iodide mixtures. Mutic et al. [352] used a FIA system with amperometric detection on a platinum electrode. The bromide determination was based on its oxidation to bromine, using potassium permanganate as oxidizing agent. The generated bromine diffuses through PTFE membrane into the sulfuric acid acceptor flow in a gas-diffusion unit. The acceptor flow carried bromine to an amperometric detector. The authors describe very good limit of detection of $50 \mathrm{nM}$ and two linearities from $2 \mu \mathrm{M}$ to $100 \mu \mathrm{M}$ and from $0.1 \mathrm{mM}$ to $10 \mathrm{mM}$. The major disadvantages of this work are the highly complicated sample pretreatment, the use of very aggressive reagents, the complicated mounting scheme and the equipment required for the analysis, which prohibit an in situ use.

Similar to the observed for chloride (Section 3.7.2.2.), the use of silver permitted the study of the halide onto electrode surfaces [353]. Recently published works, showed the suitability of SPCEs modified with Ag-Nps [44] and of SPAgE [104] to perform bromide determinations in waters. Still, electrode fouling, impractical continuous measurement, and the reduced analysis ranges obtained, are challenges to overcome with these approaches.

Moreover, out of the analytical scope, Benett et al. [354] performed an electrochemical study of the voltammetric behavior of the redox pair $\mathrm{Br}^{-} / \mathrm{Br}_{2}$ in non-aqueous solvents. Other approaches, such as wastewater treatment, were designed to allow the selective oxidation of bromide in brines from hydraulic fracturing [323].

\subsubsection{IODIDE}

Iodide $\left(\mathrm{I}^{-}\right)$, also a halide anion, have a close relationship with the bromide. Likewise, the presence of iodide in potable water, also outcome in potentially carcinogenic iodates, formed during the oxidizing disinfection steps. Therefore, it is an important task the monitoring of this anion in drinking waters [328]. 
Notwithstanding, iodide plays an important role in biological processes, being an essential element in human nutrition. In the organism, iodide is present in minimal amounts, mainly in the thyroid gland, where is crucial for the synthesis of thyroid stimulating hormone (TSH). The synthesis of such hormones is affected when iodide requirements are not met, resulting in iodide deficiency diseases (IDD) such as goiter, hypothyroidism (low level of thyroid hormones in the blood) [355-357].

Goiter, characterized by the swelling in the neck, is the most visible manifestation of IDD representing a maladaption to the anion deficiency. The swelling results from an increased stimulation of the thyroid gland by the TSH, aiming to use the maximum of available iodide [358]. However, the most damaging IDDs are the irreversible mental retardation and cretinism (congenital iodine deficiency syndrome), which can emerge across the life-span, from the fetal stage to adult age. Particularly in the case of young children, if a deficit of iodide occurs between the fetal stage and the third month after birth, which is the period for brain development; miscarriage, perinatal mortality and irreversible mental retardation can occur $[355,357]$. The deficit of iodide during women pregnancy, is also correlated with attention deficit hyperactivity disorders in their children [359]. As well as, abnormal protein synthesis and energy transfer regulation, a decrease in individuals' growth and development, and effects in the central nervous system structure, have been also associated as a consequence of the poor iodide levels [360].

Foods like bread, milk [361], seafoods and edible plants [360], have been recognized as sources of iodide. However, for populations that living in areas where the soil has a low iodide content, the crops do not provide adequate amounts of this nutrient when consumed [355]. With recommended daily intake of $150 \mu \mathrm{g}$ for a regular adult, $250 \mu \mathrm{g}$ for pregnant women and 90 to $120 \mu \mathrm{g}$ for children aged between 1 to 13 years, the IDDs prevalence in these populations is a major concern $[357,362]$.

The WHO considered iodide deficiency a public health problem, categorizing the IDDs as an epidemiologic disease and recommending actuations of food iodization to correct the populations iodide levels, mostly through salt iodization [355]. Also pharmaceutical supplementation, using multivitamin pills, were also widely applied [355,357,363]. 
Moreover, the edible plants biofortification was also proposed, by fertilizing the crops with algal-based iodized organic fertilizer [360].

The IDDs occurrence was first measured regarding the high prevalence of goiter in the resident populations. However, this shows to be of limited use to access the impact of food iodization, exhibiting a lack of sensitivity in acute changes of iodide intake. Therefore, WHO indicated the urinary iodide, as preferable and most useful indicator of IDDs, since reflected the dietary intake. In fact, approximately $90 \%$ of ingested iodide is excreted in urine, which means that a lower fraction of urinary iodide (UI) is a result of an inadequate intake; and consequently, the IDD risk exist. Therefore, UI is a sensitive indicator of changes in iodide consumption across the population and is already used as tool for measuring IDDs prevalence [355,357,362].

According to the WHO's guidelines, a UI lower than $0.32 \mu \mathrm{M}$ indicate a severe deficiency of the individuals, since the considered optimal levels are between a range from 1.58 and $3.14 \mu \mathrm{M}$. In the case of pregnant women the adequate levels are known between 2.36 and $3.92 \mu \mathrm{M}$ for UI, and in some cases, can be higher than $7.88 \mu \mathrm{M}$ [357].

Different sources that contribute for UI levels are the radioactive iodine $\left({ }^{131} \mathrm{I}\right)$ and the intravenous iodinated contrast materials, both used in the treatment and diagnosis of differentiated carcinoma [364-368]; and also, the iodide from other pharmaceutical formulations, highlighting the significance of urine as matrix for iodide determination.

\subsubsection{Analytical determination of iodide}

The relationship between iodine assimilation and intake, is highly affected by the ubiquitous environmental presence of the anion, trouble the assessing of the nutrition status of the populations. Several techniques have been proposed to quantify iodide in samples of very different nature, like soils, plants, various foods and in physiological samples. Numerous analytical approaches have been proposed for quantification of iodide, however, only a few are currently used as routine analysis [369]. 
Considered as the analytical standards for iodide measurements, ICP-MS [370] and spectrophotometric method of Sandell-Kolthoff reaction (SK) kinetic measurement [371], are nowadays the most used methods in clinical and industry fields.

In particular the SK spectrophotometric method, shows viability for biological samples. The method is based in the catalytic effect of iodide anions on the reaction rate between $\mathrm{Ce}^{4+}$ and $\mathrm{As}^{3+}$, following the equations 3.15 and 3.16.

$$
\begin{aligned}
& 2 C e^{4+}+2 I^{-} \rightarrow 2 C e^{3+}+I_{2} \\
& A s^{3+}+I_{2} \rightarrow A s^{5+}+2 I^{-}
\end{aligned}
$$

The $\mathrm{Ce}^{4+}$ exhibit a yellow coloration $(\lambda=370 \mathrm{~nm})$, which is converted into colorless solution due to the presence $\mathrm{Ce}^{3+}$ (colorless). The $\mathrm{Ce}^{4+}$ reduction promoted by $\mathrm{As}^{3+}$, is normally very slow, however, trace amounts of iodide catalyze the rate kinetic, allowing the halide monitoring with the time [371]. The SK spectrophotometric method is recognized to be highly susceptible to interferences, requiring an exhaustive sample pretreatment before the measurements.

On the other hand, the ICP-MS offer a superb sensitivity, nevertheless, it is still necessary to perform sample pretreatment steps, adding the requirement of an internal standard to account for matrix effects [370].

Alternative methods, such as atomic absorption spectrometry [372], optical emission spectrometry [373], neutron activation analysis [374], flow injection analysis [47], gas and liquid chromatographic methods [375,376], UV-visible spectrometry [377], X-ray fluorescence [378], radiotracer analysis by scintillation counting [379], and quartz crystal oscillator [380], have been described to improve the iodide determinations.

Once again, the routine use of these techniques seem to be limited to laboratory facilities, not meeting the in situ requirement that exist nowadays. Moreover, the majority of them require expensive instrumentation and qualified personnel, as well as, complex sample pretreatments prior to analysis. 


\subsubsection{Electrochemical determinations of iodide}

Electrochemical methods are an efficient alternative to these techniques, meeting the needs of developing countries and allowing the development of new, inexpensive and reliable methods to perform iodide determinations.

As described for the other halides and presenting the same characteristics, potentiometric iodide ion-selective electrodes (I-ISE) have shown its applicability for iodide analysis [381,382]. In light of this, the use of voltammetric and amperometric techniques for iodide detection, seems to overpass some of the known drawbacks.

Several conventional electrodes modifications, were carried out to improve the selectivity and sensitivity of these probes for iodide determination in numerous matrices. Systems based on glassy carbon electrodes [146,383-389], carbon paste electrodes [383,390], carbon electrode generated by electrospinning [391], gold electrodes [84,392,393], platinum electrodes [394-397] and also fluorine-doped tin oxide electrodes [396], have been discussed in the literature. Once again, silver shows to play an important role in halide anions determinations, with the use of conventional silver working electrodes cited several times [398-403]. Notwithstanding, the unpractical use of these platforms as point-of-care, the increased difficulty to modified and reuse the electrodic surfaces, must be considered when using these probes. Additionally, in case of silver electrodes, the AgI deposits formation results in the sensitivity loss of the devices, requiring extra cleaning steps between each measurement.

Regarding that, the use of SPEs for the development of iodide sensors has been the preferable choice among several authors. The SPEs functioning based in the use of silver [44,104], gold and platinum [404] can be found. The use of an ionic liquid interface between the electrode surface and the iodide solution, was also explored to increase the selectivity of the devices towards iodide anions [405,406]. Moreover, a modified copper (II)-sulphamethazine Schiff base complex SPCE [390] was proposed to enhance the 
selectivity for potentiometric determination of the anion, forming a iodide selective membrane onto the WE surface.

The need for complicated electrode modifications, and in some cases, a complex sample pretreatment; once again limit their use as point-of-care methods. However, a few SPEsbased approaches, use CS modifications onto WE surfaces, developing devices with enhanced sensitivity towards iodide anions [84,85,146,407]. CS contains cationic amino groups, which can be used to preconcentrate the iodide on the electrode interfaces [84,85,146,407]. This modification shows to be very simple and effective to perform iodide determinations, exhibiting good sensitivities and limits of detection of the devices.

Despite the promising results of the previous stated SPEs-based approaches, none of the referred works have been successfully used to determine iodide content in human urine, existing a gap for this analysis.

\subsection{REFERENCES}

[1] F.-G. Banica, Chemical sensors and biosensors - Fundamentals and applications, 1st ed., John Wiley \& Sons, Inc., UK, 2012.

[2] J. Wang, Analytical Electrochemistry, 3rd ed., John Wiley \& Sons, 2006.

[3] J.P. Hart, A. Crew, E. Crouch, K.C. Honeychurch, R.M. Pemberton, Some Recent Designs and Developments of Screen-Printed Carbon Electrochemical Sensors/Biosensors for Biomedical, Environmental, and Industrial Analyses, Anal. Lett. 37 (2004) 789-830.

[4] A. Hulanicki, S. GLab, F. Ingman, Chemical sensors definitions and classification, Pure Appl. Chem. 63 (1991) 1247-1250.

[5] D.R. Thévenot, K. Toth, R.A. Durst, G.S. Wilson, Technical report Electrochemical biosensors: recommended definitions and classification, 16 (2001) 121-131. 
[6] S. Zhang, G. Wright, Y. Yang, Materials and techniques for electrochemical biosensor design and construction., Biosens. Bioelectron. 15 (2000) 273-82.

[7] D. Grieshaber, R. MacKenzie, J. Vörös, E. Reimhult, Electrochemical Biosensors Sensor Principles and Architectures, Sensors. 8 (2008) 1400-1458.

[8] M.A. Alonso-Lomillo, O. Domínguez-Renedo, M.J. Arcos-Martinez, Enzyme modified screen printed electrodes, in: R. Courneaux, P. Novotny (Eds.), Biosens. Prop. Mater. Appl., Nova Publishers, Hauppauge NY, 2009: pp. 1-52.

[9] J.M.S. Cabral, M.R. Aires-Barros, M. Gama, Engenharia Enzimática, 1st ed., LIDEL - Edições Técnicas Lda., Lisbon, 2003.

[10] A.J. Bard, L.R. Faulkner, Electrochemical Methods - Fundamentals and Applications, 2nd Ed., John Wiley \& Sons, Inc., 2001.

[11] D.B. Hibbert, Introduction to electrochemistry, Palgrave, London, 1993.

[12] Z.G. Cynthia, Handbook of Electrochemistry, 2007.

[13] B. Molinero-Abad, D. Izquierdo, L. Pérez, I. Escudero, M.J. Arcos-Martínez, Comparison of backing materials of screen printed electrochemical sensors for direct determination of the sub-nanomolar concentration of lead in seawater, Talanta. 182 (2018) $549-557$.

[14] B. Molinero-Abad, L. Perez, D. Izquierdo, I. Escudero, M.J. Arcos-Martinez, Sensor system based on flexible screen-printed electrodes for electrochemical detection of okadaic acid in seawater, Talanta. 192 (2019) 347-352.

[15] M. Javanbakht, F. Divsar, A. Badiei, F. Fatollahi, Y. Khaniani, M.R. Ganjali, P. Norouzi, M. Chaloosi, G.M. Ziarani, Determination of picomolar silver concentrations by differential pulse anodic stripping voltammetry at a carbon paste electrode modified with phenylthiourea-functionalized high ordered nanoporous silica gel, Electrochim. Acta. 54 (2009) 5381-5386. 
[16] M. Zhou, J.E. Dick, K. Hu, M. V. Mirkin, A.J. Bard, Ultrasensitive Electroanalysis: Femtomolar Determination of Lead, Cobalt, and Nickel, Anal. Chem. 90 (2018) 11421146.

[17] K.M. Hanson, T.J. Pappas, L.A. Holland, Electrochemical detection in capillary electrophoresis, in: Compr. Anal. Chem., 2005: pp. 413-440.

[18] D.A.C. Brownson, C.E. Banks, The Handbook of Graphene Electrochemistry, in: Handb. Graphene Electrochem., 2014: pp. 23-77.

[19] J.E.B. Randles, A cathode ray polarograph. Part II-The current-voltage curves., Transac Far Soc. 44 (1947) 327-338.

[20] A. Ševcík, Oscillographic polarography with periodical triangular voltage, Collect. Czech. Chem. Commun. 13 (1948) 349-377.

[21] P.T. Kissinger, W.R. Heineman, Cyclic Voltammetry, J. Chem. Educ. 60 (1983) $702-706$.

[22] A. García-Miranda Ferrari, C.W. Foster, P.J. Kelly, D.A.C. Brownson, C.E. Banks, Determination of the Electrochemical Area of Screen-Printed Electrochemical Sensing Platforms, Biosensors. 8 (2018) 1-10.

[23] D.A. Skoog, D.M. West, F.J. Holler, S.R. Crouch, Fundamentals of Analytical Chemistry, 8th Editio, Thomson Brooks/Cole, Australia, 2004.

[24] S. Alegret, A. Merkoci, Comprehensive Analytical Chemistry: Electrochemical Sensor Analysis, Elsevier. 49 (2007) 1308.

[25] O.D. Renedo, M.A. Alonso-Lomillo, M.J.A. Martínez, Recent developments in the field of screen-printed electrodes and their related applications., Talanta. 73 (2007) 20219. 
[26] M. Tudorache, C. Bala, Biosensors based on screen-printing technology, and their applications in environmental and food analysis., Anal. Bioanal. Chem. 388 (2007) 56578.

[27] J.P. Metters, R.O. Kadara, C.E. Banks, New directions in screen printed electroanalytical sensors: an overview of recent developments., Analyst. 136 (2011) 106776.

[28] H. Cunha-Silva, Disposable immunosensor for diagnosis of Human Cytomegalovirus infection - Antibodies immobilization, Universidade da Beira Interior (Covilhã-Portugal),2014.

https://ubibliorum.ubi.pt/bitstream/10400.6/5633/1/3524_7166.pdf.

[29] M. Li, Y.T. Li, D.W. Li, Y.T. Long, Recent developments and applications of screen-printed electrodes in environmental assays-A review, Anal. Chim. Acta. 734 (2012) $31-44$.

[30] M. Li, D.W. Li, G. Xiu, Y.T. Long, Applications of screen-printed electrodes in current environmental analysis, Curr. Opin. Electrochem. 3 (2017) 137-143.

[31] M.L.Y. Sin, K.E. Mach, P.K. Wong, J.C. Liao, Advances and challenges in biosensor-based diagnosis of infectious diseases., Expert Rev. Mol. Diagn. 14 (2014) 22544.

[32] W.J. Paschoalino, S. Kogikoski, J.T.C. Barragan, J.F. Giarola, L. Cantelli, T.M. Rabelo, T.M. Pessanha, L.T. Kubota, Emerging Considerations for the Future Development of Electrochemical Paper-Based Analytical Devices, ChemElectroChem. (2018) 10-30.

[33] J. Kim, G. Valdés-Ramírez, A.J. Bandodkar, W. Jia, A.G. Martinez, J. Ramírez, P. Mercier, J. Wang, Non-invasive mouthguard biosensor for continuous salivary monitoring of metabolites, Analyst. 139 (2014) 1632-6. 
[34] D. Khodagholy, V.F. Curto, K.J. Fraser, M. Gurfinkel, R. Byrne, D. Diamond, G.G. Malliaras, F. Benito-Lopez, R.M. Owens, Organic electrochemical transistor incorporating an ionogel as a solid state electrolyte for lactate sensing, J. Mater. Chem. 22 (2012) 4440.

[35] S. Emaminejad, W. Gao, E. Wu, Z.A. Davies, H. Yin Yin Nyein, S. Challa, S.P. Ryan, H.M. Fahad, K. Chen, Z. Shahpar, S. Talebi, C. Milla, A. Javey, R.W. Davis, Autonomous sweat extraction and analysis applied to cystic fibrosis and glucose monitoring using a fully integrated wearable platform, Proc. Natl. Acad. Sci. 114 (2017) $4625-4630$.

[36] V.A.T. Dam, M.A.G. Zevenbergen, R. van Schaijk, Toward wearable patch for sweat analysis, Sensors Actuators, B Chem. 236 (2016) 834-838.

[37] V.A.T. Dam, M. Goedbloed, M.A.G. Zevenbergen, Solid-Contact Reference Electrode for Ion-Selective Sensors, Proceedings. 1 (2017) 464.

[38] W. Gao, S. Emaminejad, H.Y.Y. Nyein, S. Challa, K. Chen, A. Peck, H.M. Fahad, H. Ota, H. Shiraki, D. Kiriya, D.-H. Lien, G.A. Brooks, R.W. Davis, A. Javey, Fully integrated wearable sensor arrays for multiplexed in situ perspiration analysis, Nature. 529 (2016) 509-514.

[39] A. Abellán-Llobregat, I. Jeerapan, A. Bandodkar, L. Vidal, A. Canals, J. Wang, E. Morallón, A stretchable and screen-printed electrochemical sensor for glucose determination in human perspiration, Biosens. Bioelectron. 91 (2017) 885-891.

[40] W. Jia, A.J. Bandodkar, G. Valde-Ramírez, J.R. Windmiller, Z. Yang, J. Ramírez, G. Chan, J. Wang, Electrochemical tattoo biosensors for real-time noninvasive lactate monitoring in human perspiration, Anal. Chem. 85 (2013) 6553-6560.

[41] S. Anastasova, B. Crewther, P. Bembnowicz, V. Curto, H.M. Ip, B. Rosa, G.Z. Yang, A wearable multisensing patch for continuous sweat monitoring, Biosens. Bioelectron. 94 (2017) 730. 
[42] S. Imani, A.J. Bandodkar, A.M.V. Mohan, R. Kumar, S. Yu, J. Wang, P.P. Mercier, A wearable chemical-electrophysiological hybrid biosensing system for real-time health and fitness monitoring, Nat. Commun. 7 (2016) 11650.

[43] J. Bujes-Garrido, M.J. Arcos-Martínez, Development of a wearable electrochemical sensor for voltammetric determination of chloride ions, Sensors Actuators, B Chem. 240 (2017) 224-228.

[44] J. Bujes-Garrido, D. Izquierdo-Bote, A. Heras, A. Colina, M.J. Arcos-Martínez, Determination of halides using Ag nanoparticles-modified disposable electrodes. A first approach to a wearable sensor for quantification of chloride ions, Anal. Chim. Acta. 1012 (2018) 42-48.

[45] H. Cunha-Silva, M. Julia Arcos-Martinez, Development of a selective chloride sensing platform using a screen-printed platinum electrode, Talanta. 195 (2019) 771-777.

[46] G. Matzeu, L. Florea, D. Diamond, Advances in wearable chemical sensor design for monitoring biological fluids, Sensors Actuators, B Chem. 211 (2015) 403-418.

[47] V. V. Kuznetsov, Y. V. Ermolenko, L. Seffar, Amylose and amylopectin as reagents for the flow-injection determination of elemental iodine, J. Anal. Chem. 62 (2007) 479485.

[48] J.R. Windmiller, J. Wang, Wearable Electrochemical Sensors and Biosensors: A Review, Electroanalysis. 25 (2013) 29-46.

[49] M. Mehrvar, M. Abdi, Recent developments, characteristics, and potential applications of electrochemical biosensors., Anal. Sci. 20 (2004) 1113-26.

[50] M.P. O’Halloran, M. Pravda, G.G. Guilbault, Prussian Blue bulk modified screenprinted electrodes for $\mathrm{H} 2 \mathrm{O} 2$ detection and for biosensors, Talanta. 55 (2001) 605-611. 
[51] L. Asturias-Arribas, M. Asunción Alonso-Lomillo, O. Domínguez-Renedo, M. Julia Arcos-Martínez, Screen-printed biosensor based on the inhibition of the acetylcholinesterase activity for the determination of codeine, Talanta. 111 (2013) 8-12.

[52] L. Asturias-Arribas, M.A. Alonso-Lomillo, O. Domínguez-Renedo, M.J. ArcosMartínez, Electrochemical determination of cocaine using screen-printed cytochrome P450 2B4 based biosensors, Talanta. 105 (2013) 131-134.

[53] A. Calvo-Pérez, O. Domínguez-Renedo, M.A. Alonso-Lomillo, M.J. ArcosMartínez, Disposable Horseradish Peroxidase Biosensors for the Selective Determination of Tyramine, Electroanalysis. 25 (2013) 1316-1322.

[54] A. Chen, S. Chatterjee, Nanomaterials based electrochemical sensors for biomedical applications, Chem. Soc. Rev. 42 (2013) 5425.

[55] A.J.S. Ahammad, J.-J. Lee, M.A. Rahman, Electrochemical Sensors Based on Carbon Nanotubes, Sensors. 9 (2009) 2289-2319.

[56] A.C. Power, B. Gorey, S. Chandra, J. Chapman, Carbon nanomaterials and their application to electrochemical sensors: A review, Nanotechnol. Rev. 7 (2018) 19-41.

[57] B.R. Adhikari, M. Govindhan, A. Chen, Carbon nanomaterials based electrochemical sensors/biosensors for the sensitive detection of pharmaceutical and biological compounds, Sensors (Switzerland). 15 (2015) 22490-22508.

[58] S. Cinti, F. Arduini, Graphene-based screen-printed electrochemical (bio)sensors and their applications: Efforts and criticisms, Biosens. Bioelectron. 89 (2017) 107-122.

[59] X. Xu, D. Huang, K. Cao, M. Wang, S.M. Zakeeruddin, M. Grätzel, Electrochemically reduced graphene oxide multilayer films as efficient counter electrode for dye-sensitized solar cells, Sci. Rep. 3 (2013). 
[60] X. Cai, J. Yan, H. Chu, M. Wu, Y. Tu, An exercise degree monitoring biosensor based on electrochemiluminescent detection of lactate in sweat, Sensors Actuators, B Chem. 143 (2010) 655-659.

[61] R. Tan, D. Wu, S. Xu, Y. Zhu, D. Xiong, L. Wang, P. Yang, P.K. Chu, Electrocatalytic hydrogen evolution of palladium nanoparticles electrodeposited on nanographene coated macroporous electrically conductive network, Int. J. Hydrogen Energy. 43 (2018) 2171-2183.

[62] S.K. Vashist, D. Zheng, K. Al-Rubeaan, J.H.T. Luong, F.S. Sheu, Advances in carbon nanotube based electrochemical sensors for bioanalytical applications, Biotechnol. Adv. 29 (2011) 169-188.

[63] W. Gao, H. Dong, J. Lei, H. Ji, H. Ju, Signal amplification of streptavidinhorseradish peroxidase functionalized carbon nanotubes for amperometric detection of attomolar DNA., Chem. Commun. (Camb). 47 (2011) 5220-2.

[64] Y.C. Tsai, S.Y. Chen, C.A. Lee, Amperometric cholesterol biosensors based on carbon nanotube-chitosan-platinum-cholesterol oxidase nanobiocomposite, Sensors Actuators, B Chem. 135 (2008) 96-101.

[65] J. Weber, A. Kumar, A. Kumar, S. Bhansali, Novel lactate and pH biosensor for skin and sweat analysis based on single walled carbon nanotubes, Sensors Actuators, B Chem. 117 (2006) 308-313.

[66] H. Teymourian, A. Salimi, R. Hallaj, Low potential detection of NADH based on $\mathrm{Fe} 3 \mathrm{O} 4$ nanoparticles/multiwalled carbon nanotubes composite: Fabrication of integrated dehydrogenase-based lactate biosensor, Biosens. Bioelectron. 33 (2012) 60-68.

[67] I.M. Apetrei, C. Apetrei, The biocomposite screen-printed biosensor based on immobilization of tyrosinase onto the carboxyl functionalised carbon nanotube for assaying tyramine in fish products, J. Food Eng. 149 (2015) 1-8. 
[68] H. Tang, J. Chen, S. Yao, L. Nie, G. Deng, Y. Kuang, Amperometric glucose biosensor based on adsorption of glucose oxidase at platinum nanoparticle-modified carbon nanotube electrode, Anal. Biochem. 331 (2004) 89-97.

[69] M. Briones, E. Casero, M.D. Petit-Domínguez, M.A. Ruiz, A.M. Parra-Alfambra, F. Pariente, E. Lorenzo, L. Vázquez, Diamond nanoparticles based biosensors for efficient glucose and lactate determination, Biosens. Bioelectron. 68 (2015) 521-528.

[70] O. a. Loaiza, P.J. Lamas-Ardisana, L. Añorga, E. Jubete, V. Ruiz, M. Borghei, G. Cabañero, H.J. Grande, Graphitized carbon nanofiber-Pt nanoparticle hybrids as sensitive tool for preparation of screen printing biosensors. Detection of lactate in wines and ciders, Bioelectrochemistry. 101 (2015) 58-65.

[71] D. Dogrukol-Ak, V. Zaimoglu, M. Tunçel, Voltammetry of trazodone by platinum electrode and its determination in tablets using DP technique in the rotating conditions, Eur. J. Pharm. Sci. 7 (1999) 215-220.

[72] B. Xue, Y. Fu, J. Li, E. Liu, Voltammetric Determination of Serine Using LCysteine Modified Platinum Electrode and Brief Exploration from the Theoretical Perspective, Electroanalysis. 30 (2018) 1060-1065.

[73] L.S. De Oliveira, M.A. Balbino, M.M.T. De Menezes, E.R. Dockal, M.F. De Oliveira, Voltammetric analysis of cocaine using platinum and glassy carbon electrodes chemically modified with Uranyl Schiff base films, Microchem. J. 110 (2013) 374-378.

[74] R.K. Shervedani, A. Hatefi-Mehrjardi, A. Asadi-Farsani, Sensitive determination of iron(III) by gold electrode modified with 2-mercaptosuccinic acid self-assembled monolayer, Anal. Chim. Acta. 601 (2007) 164-171.

[75] R.N. Goyal, V.K. Gupta, N. Bachheti, R.A. Sharma, Electrochemical sensor for the determination of dopamine in presence of high concentration of ascorbic acid using a fullerene-C60coated gold electrode, Electroanalysis. 20 (2008) 757-764. 
[76] G. Chen, H. Shi, F. Ban, Y. Zhang, L. Sun, Determination of trypsin activity using a gold electrode modified with a nanocover composed of graphene oxide and thionine, Microchim. Acta. 182 (2015) 2469-2476.

[77] C. Giuriati, S. Cavalli, A. Gorni, D. Badocco, P. Pastore, Ion chromatographic determination of sulfide and cyanide in real matrices by using pulsed amperometric detection on a silver electrode, J. Chromatogr. A. 1023 (2004) 105-112.

[78] M. Brand, I. Eshkenazi, E. Kirowa-Eisner, The Silver Electrode in Square-Wave Anodic Stripping Voltammetry. Determination of $\mathrm{Pb}^{2+}$ without Removal of Oxygen, Anal. Chem. 69 (1997) 4660-4664.

[79] J. Wang, Electrochemical biosensing based on noble metal nanoparticles, Microchim. Acta. 177 (2012) 245-270.

[80] J.-J. Xu, W.-W. Zhao, S. Song, C. Fan, H.-Y. Chen, Functional nanoprobes for ultrasensitive detection of biomolecules: an update., Chem. Soc. Rev. 43 (2014) 1601-11.

[81] R.A.S. Couto, J.L.F.C. Lima, M.B. Quinaz, Recent developments, characteristics and potential applications of screen-printed electrodes in pharmaceutical and biological analysis, Talanta. 146 (2016) 801-814.

[82] Y. Xu, E. Wang, Electrochemical biosensors based on magnetic micro/nano particles, Electrochim. Acta. 84 (2012) 62-73.

[83] E. Bernalte, C. Marín Sánchez, E. Pinilla Gil, Gold nanoparticles-modified screenprinted carbon electrodes for anodic stripping voltammetric determination of mercury in ambient water samples, Sensors Actuators, B Chem. 161 (2012) 669-674.

[84] Y. Zhang, M. Wang, Chitosan/Colloid Au Modified Gold Film Microelectrode for Iodide Determination, Int. J. Electrochem. Sci. 8 (2013) 4595-4609. 
[85] K.M. Kim, Y.S. Nam, Y. Lee, K.B. Lee, Highly sensitive and selective assay method for iodide ion determination based on gold nanoparticles conjugated with glycol chitosan, Int. J. Environ. Anal. Chem. 97 (2017) 673-683.

[86] F. Pires, H. Silva, O. Domínguez-Renedo, M.A. Alonso-Lomillo, M.J. ArcosMartínez, A.C. Dias-Cabral, Disposable immunosensor for human cytomegalovirus glycoprotein B detection, Talanta. 136 (2015) 42-46.

[87] V. V. Shumyantseva, S. Carrara, V. Bavastrello, D.J. Riley, T. V. Bulko, K.G. Skryabin, A.I. Archakov, C. Nicolini, Direct electron transfer between cytochrome P450scc and gold nanoparticles on screen-printed rhodium-graphite electrodes, Biosens. Bioelectron. 21 (2005) 217-222.

[88] O. Shulga, J.R. Kirchhoff, An acetylcholinesterase enzyme electrode stabilized by an electrodeposited gold nanoparticle layer, Electrochem. Commun. 9 (2007) 935-940.

[89] V. Carralero Sanz, M.L. Mena, A. González-Cortés, P. Yáñez-Sedeño, J.M. Pingarrón, Development of a tyrosinase biosensor based on gold nanoparticles-modified glassy carbon electrodes, Anal. Chim. Acta. 528 (2005) 1-8.

[90] J.-A.A. Ho, W.-L. Hsu, W.-C. Liao, J.-K. Chiu, M.-L. Chen, H.-C. Chang, C.-C. Li, Ultrasensitive electrochemical detection of biotin using electrically addressable siteoriented antibody immobilization approach via aminophenyl boronic acid., Biosens. Bioelectron. 26 (2010) 1021-7.

[91] R. Narayanan, M.A. El-Sayed, Shape-dependent catalytic activity of platinum nanoparticles in colloidal solution, Nano Lett. 4 (2004) 1343-1348.

[92] X. Dai, R.G. Compton, Detection of As(III) via oxidation to As(V) using platinum nanoparticle modified glassy carbon electrodes: arsenic detection without interference from copper, Analyst. 131 (2006) 516-521. 
[93] S. Sanllorente-Méndez, O. Domínguez-Renedo, M.J. Arcos-Martínez, Determination of arsenic(III) using platinum nanoparticle-modified screen-printed carbonbased electrodes, Electroanalysis. 21 (2009) 635-639.

[94] R.T. Kushikawa, M.R. Silva, A.C.D. Angelo, M.F.S. Teixeira, Construction of an electrochemical sensing platform based on platinum nanoparticles supported on carbon for tetracycline determination, Sensors Actuators, B Chem. 228 (2016) 207-213.

[95] S. Shahrokhian, N. Hosseini-Nassab, M. Ghalkhani, Construction of Pt nanoparticle-decorated graphene nanosheets and carbon nanospheres nanocompositemodified electrodes: Application to ultrasensitive electrochemical determination of cefepime, RSC Adv. 4 (2014) 7786-7794.

[96] P.K. Kalambate, B.J. Sanghavi, S.P. Karna, A.K. Srivastava, Simultaneous voltammetric determination of paracetamol and domperidone based on a graphene/platinum nanoparticles/nafion composite modified glassy carbon electrode, Sensors Actuators, B Chem. 213 (2015) 285-294.

[97] N. Liu, F. Feng, Z. Liu, Z. Ma, Porous platinum nanoparticles and PdPt nanocages for use in an ultrasensitive immunoelectrode for the simultaneous determination of the tumor markers CEA and AFP, Microchim. Acta. 182 (2015) 1143-1151.

[98] K. Murugappan, D.W.M. Arrigan, D.S. Silvester, Electrochemical Behavior of Chlorine on Platinum Microdisk and Screen-Printed Electrodes in a Room Temperature Ionic Liquid, J. Phys. Chem. C. 119 (2015) 23572-23579.

[99] S.B. Hall, E.A. Khudaish, A.L. Hart, Electrochemical oxidation of hydrogen peroxide at platinum electrodes. Part 1. An adsorption-controlled mechanism, Electroanalysis. 43 (1998) 579-588.

[100] H. Wu, J. Wang, X. Kang, C. Wang, D. Wang, J. Liu, I.A. Aksay, Y. Lin, Glucose biosensor based on immobilization of glucose oxidase in platinum nanoparticles/graphene/chitosan nanocomposite film, Talanta. 80 (2009) 403-406. 
[101] X. Jiang, Y. Wu, X. Mao, X. Cui, L. Zhu, Amperometric glucose biosensor based on integration of glucose oxidase with platinum nanoparticles/ordered mesoporous carbon nanocomposite, Sensors Actuators, B Chem. 153 (2011) 158-163.

[102] J. Huang, J. Li, Y. Yang, X. Wang, B. Wu, J. ichi Anzai, T. Osa, Q. Chen, Development of an amperometric L-lactate biosensor based on L-lactate oxidase immobilized through silica sol-gel film on multi-walled carbon nanotubes/platinum nanoparticle modified glassy carbon electrode, Mater. Sci. Eng. C. 28 (2008) 1070-1075.

[103] F. Shahdost-fard, A. Salimi, S. Khezrian, Highly selective and sensitive adenosine aptasensor based on platinum nanoparticles as catalytical label for amplified detection of biorecognition events through H2O2reduction, Biosens. Bioelectron. 53 (2014) 355-362.

[104] M.H. Chiu, W.L. Cheng, G. Muthuraman, C.T. Hsu, H.H. Chung, J.M. Zen, A disposable screen-printed silver strip sensor for single drop analysis of halide in biological samples, Biosens. Bioelectron. 24 (2009) 3008-3013.

[105] C.M. Welch, C.E. Banks, A.O. Simm, R.G. Compton, Silver nanoparticle assemblies supported on glassy-carbon electrodes for the electro-analytical detection of hydrogen peroxide, Anal. Bioanal. Chem. 382 (2005) 12-21.

[106] W. Zhao, H. Wang, X. Qin, X. Wang, Z. Zhao, Z. Miao, L. Chen, M. Shan, Y. Fang, Q. Chen, A novel nonenzymatic hydrogen peroxide sensor based on multi-wall carbon nanotube/silver nanoparticle nanohybrids modified gold electrode, Talanta. 80 (2009) 1029-1033.

[107] J. Tashkhourian, M.R.H. Nezhad, J. Khodavesi, S. Javadi, Silver nanoparticles modified carbon nanotube paste electrode for simultaneous determination of dopamine and ascorbic acid, J. Electroanal. Chem. 633 (2009) 85-91.

[108] N. Butwong, S. Srijaranai, J.D. Glennon, J.H.T. Luong, Cysteamine Capped Silver Nanoparticles and Single-walled Carbon Nanotubes Composite Coated on Glassy Carbon 
Electrode for Simultaneous Analysis of Hydroquinone and Catechol, Electroanalysis. 30 (2018) 962-968.

[109] E. Hüger, K. Osuch, Ferromagnetism in body centred cubic Rh, Solid State Commun. 131 (2004) 175-179.

[110] M. Barquero-Quirós, M.J. Arcos-Martínez, Effect of nanoparticles on modified screen printed inhibition superoxide dismutase electrodes for aluminum, Sensors. 16 (2016).

[111] M. Barquero-quirós, H. Cunha-silva, M.J. Arcos-martínez, Superoxide Dismutase Based Biosensor for the Electrochemical Determination of Epinephrine, Acta Chim. Pharm. Indica. 8 (2018) 1-19.

[112] X. Guo, B. Liang, J. Jian, Y. Zhang, X. Ye, Glucose biosensor based on a platinum electrode modified with rhodium nanoparticles and with glucose oxidase immobilized on gold nanoparticles, Microchim. Acta. 181 (2014) 519-525.

[113] K.S. Lokesh, Y. Shivaraj, B.P. Dayananda, S. Chandra, Synthesis of phthalocyanine stabilized rhodium nanoparticles and their application in biosensing of cytochrome c, Bioelectrochemistry. 75 (2009) 104-109.

[114] G. Hu, Z. Zhou, Y. Guo, H. Hou, S. Shao, Electrospun rhodium nanoparticle-loaded carbon nanofibers for highly selective amperometric sensing of hydrazine, Electrochem. Commun. 12 (2010) 422-426.

[115] V.A. Gatselou, D.L. Giokas, A.G. Vlessidis, M.I. Prodromidis, Rhodium nanoparticle-modified screen-printed graphite electrodes for the determination of hydrogen peroxide in tea extracts in the presence of oxygen, Talanta. 134 (2015) 482-487.

[116] Z. Song, H. Chang, W. Zhu, C. Xu, X. Feng, Rhodium Nanoparticle-mesoporous Silicon Nanowire Nanohybrids for Hydrogen Peroxide Detection with High Selectivity, Sci. Rep. 5 (2015) 1-4. 
[117] U. Lange, T. Hirsch, V.M. Mirsky, O.S. Wolfbeis, Hydrogen sensor based on a graphene-palladium nanocomposite, Electrochim. Acta. 56 (2011) 3707-3712.

[118] N.P. Shetti, D.S. Nayak, S.J. Malode, R.M. Kulkarni, Electrochemical Sensor Based upon Ruthenium Doped $\mathrm{TiO}_{2}$ Nanoparticles for the Determination of Flufenamic Acid, J. Electrochem. Soc. 164 (2017) B3036-B3042.

[119] J. Wang, D.F. Thomas, A. Chen, Nonenzymatic electrochemical glucose sensor based on nanoporous PtPb networks, Anal. Chem. 80 (2008) 997-1004.

[120] Y. Cui, B. Li, H. He, W. Zhou, B. Chen, G. Qian, Metal-Organic Frameworks as Platforms for Functional Materials, Acc. Chem. Res. 49 (2016) 483-493.

[121] B. Li, H.M. Wen, Y. Cui, W. Zhou, G. Qian, B. Chen, Emerging Multifunctional Metal-Organic Framework Materials, Adv. Mater. 28 (2016).

[122] I. Stassen, N. Burtch, A. Talin, P. Falcaro, M. Allendorf, R. Ameloot, An updated roadmap for the integration of metal-organic frameworks with electronic devices and chemical sensors, Chem. Soc. Rev. 46 (2017) 3185-3241.

[123] F.Y. Yi, D. Chen, M.K. Wu, L. Han, H.L. Jiang, Chemical Sensors Based on MetalOrganic Frameworks, Chempluschem. 81 (2016) 675-690.

[124] B. Li, H.-M. Wen, Y. Cui, W. Zhou, G. Qian, B. Chen, Emerging Multifuntional MEtal-Organic Framework Materials, Adv. Mater. (2016) 8819-8860.

[125] S.E. Miller, M.H. Teplensky, P.Z. Moghadam, D. Fairen-Jimenez, Metal-organic frameworks as biosensors for luminescence-based detection and imaging, Interface Focus. 6 (2016) 20160027.

[126] H. Furukawa, K.E. Cordova, M. O’Keeffe, O.M. Yaghi, The Chemistry and Applications of Metal-Organic Frameworks, Science. 341:123044 (2013). 
[127] F.-X. Qin, S.-Y. Jia, F.-F. Wang, S.-H. Wu, J. Song, Y. Liu, Hemin@ @etal-organic framework with peroxidase-like activity and its application to glucose detection, Catal. Sci. Technol. 3 (2013) 2761.

[128] Y. Wang, Y. Wu, J. Xie, H. Ge, X. Hu, Multi-walled carbon nanotubes and metalorganic framework nanocomposites as novel hybrid electrode materials for the determination of nano-molar levels of lead in a lab-on-valve format, Analyst. 138 (2013) $5113-5120$.

[129] X. Wang, X. Lu, L. Wu, J. Chen, 3D metal-organic framework as highly efficient biosensing platform for ultrasensitive and rapid detection of bisphenol A, Biosens. Bioelectron. 65 (2015) 295-301.

[130] Y. Wang, H. Ge, G. Ye, H. Chen, X. Hu, Carbon functionalized metal organic framework/Nafion composites as novel electrode materials for ultrasensitive determination of dopamine, J. Mater. Chem. B. 3 (2015) 3747-3753.

[131] H. Hosseini, H. Ahmar, A. Dehghani, A. Bagheri, A. Tadjarodi, A.R. Fakhari, A novel electrochemical sensor based on metal-organic framework for electro-catalytic oxidation of L-cysteine, Biosens. Bioelectron. 42 (2013) 426-429.

[132] M. Gamero, M. Sosna, F. Pariente, E. Lorenzo, P.N. Bartlett, C. Alonso, Influence of macroporous gold support and its functionalization on lactate oxidase-based biosensors response, Talanta. 94 (2012) 328-334.

[133] J. Cruz, M. Kawasaki, W. Gorski, Electrode coatings based on chitosan scaffolds, Anal. Chem. 72 (2000) 680-686.

[134] V. Tsakova, R. Seeber, Conducting polymers in electrochemical sensing: factors influencing the electroanalytical signal, Anal. Bioanal. Chem. 408 (2016) 7231-7241. 
[135] O.S. Ahmad, T.S. Bedwell, C. Esen, A. Garcia-Cruz, S.A. Piletsky, Molecularly Imprinted Polymers in Electrochemical and Optical Sensors, Trends Biotechnol. (2018) 116.

[136] M. Arvand, P. Fallahi, Voltammetric determination of rivastigmine in pharmaceutical and biological samples using molecularly imprinted polymer modified carbon paste electrode, Sensors Actuators, B Chem. 188 (2013) 797-805.

[137] M. Kumar, A review of chitin and chitosan applications, React. Funct. Polym. 46 (2000) $1-27$.

[138] B. Krajewska, Application of chitin- and chitosan-based materials for enzyme immobilizations: A review, Enzyme Microb. Technol. 35 (2004) 126-139.

[139] M. Rinaudo, Chitin and chitosan: Properties and applications, Prog. Polym. Sci. 31 (2006) 603-632.

[140] G. Wang, J.J. Xu, H.Y. Chen, Z.H. Lu, Amperometric hydrogen peroxide biosensor with sol-gel/chitosan network-like film as immobilization matrix, Biosens. Bioelectron. 18 (2003) 335-343.

[141] Y. Liu, M. Wang, F. Zhao, Z. Xu, S. Dong, The direct electron transfer of glucose oxidase and glucose biosensor based on carbon nanotubes/chitosan matrix, Biosens. Bioelectron. 21 (2005) 984-988.

[142] X. Kang, J. Wang, H. Wu, I.A. Aksay, J. Liu, Y. Lin, Glucose Oxidase-graphenechitosan modified electrode for direct electrochemistry and glucose sensing, Biosens. Bioelectron. 25 (2009) 901-905.

[143] G.D. Altun, S.A. Cetinus, Immobilization of pepsin on chitosan beads, Food Chem. 100 (2007) 964-971.

[144] Ş. Akkuş Çetinus, H. Nursevin Öztop, Immobilization of catalase into chemically crosslinked chitosan beads, Enzyme Microb. Technol. 32 (2003) 889-894. 
[145] T.R.J. Holford, F. Davis, S.P.J. Higson, Recent trends in antibody based sensors, Biosens. Bioelectron. 34 (2012) 12-24.

[146] X. Wu, G. Lu, X. Zhang, X. Yao, Anodic voltammetric behavior of iodide at a chitosan-modified glassy carbon electrode, Anal. Lett. 34 (2001) 1205-1214.

[147] S. Wang, Y. Wang, Q. Min, T. Shu, X. Zhu, A. Peng, H. Ding, Simultaneous electrochemical determination of dopamine and serotonin in rat cerebrospinal fluid using screen-printed electrode modified with $\mathrm{MWNTs}-\mathrm{SiO}_{2}$-chitosan composites, Int. J. Electrochem. Sci. 11 (2016) 2360-2376.

[148] A. Koyun, E. Ahlatcioğlu, Y.K. İpek, Biosensors and Their Principles, in: S. Kara (Ed.), A Roadmap Biomed. Eng. Milestones, InTech, 2012: pp. 115-143.

[149] L. Cao, Carrier-bound Immobilized Enzymes: principles, applications and design, WILEY-VCH Verlag, Weinheim, Germany, 2005.

[150] L.C. Clark Jr., C. Lyons, Electrode systems for continuous monitoring in cardiovascular surgery, Ann. N. Y. Acad. Sci. 102 (1962) 29-45.

[151] F. Arduini, A. Amine, Biosensors based on enzyme inhibition, in: M. Gu, H. Kim (Eds.), Biosens. Based Aptamers Enzym., Springer, Berlin, Heidelberg, 2013: pp. 299-326.

[152] I. Taurino, R. Reiss, M. Richter, M. Fairhead, L. Thöny-Meyer, G. De Micheli, S. Carrara, Comparative study of three lactate oxidases from Aerococcus viridans for biosensing applications, Electrochim. Acta. 93 (2013) 72-79.

[153] R. Baronas, J. Kulys, A. Lančinskas, A. Žilinskas, Effect of diffusion limitations on multianalyte determination from biased biosensor response, Sensors (Switzerland). 14 (2014) 4634-4656.

[154] J. Kulys, Biosensor Response at Mixed Enzyme Kinetics and External Diffusion Limitation in Case of Substrate Inhibition, Nonlinear Anal. Model. Control. 11 (2006) 385392. 
[155] D. Simelevicius, R. Baronas, Mechanisms controlling the sensitivity of amperometric biosensors in flow injection analysis systems, J. Math. Chem. 49 (2011) $1521-1534$.

[156] G. Volpe, D. Moscone, F. Ricci, S. Piermarini, G. Palleschi, F. Arduini, L. Micheli, Electrochemical biosensors based on nanomodified screen-printed electrodes: Recent applications in clinical analysis, TrAC Trends Anal. Chem. 79 (2016) 114-126.

[157] M. Albareda-Sirvent, A. Merkoçi, S. Alegret, Configurations used in the design of screen-printed enzymatic biosensors. A review, Sensors Actuators, B Chem. 69 (2000) $153-163$.

[158] Y. Tan, Y. Fu, Q. Xie, D. Yang, H. Xiao, S. Yao, C. Chen, Recent advances in electrochemical glucose biosensors: a review, RSC Adv. 3 (2012) 4473.

[159] L. Farzin, M. Shamsipur, L. Samandari, S. Sheibani, Advances in the design of nanomaterial-based electrochemical affinity and enzymatic biosensors for metabolic biomarkers: A review, Microchim. Acta. 185 (2018).

[160] A. Amine, F. Arduini, D. Moscone, G. Palleschi, Recent advances in biosensors based on enzyme inhibtion, Biosens. Bioelectron. 76 (2015) 180-194.

[161] A. Amine, H. Mohammadi, I. Bourais, G. Palleschi, Enzyme inhibition-based biosensors for food safety and environmental monitoring, Biosens. Bioelectron. 21 (2006) $1405-1423$.

[162] G.L. Turdean, Design and Development of Biosensors for the Detection of Heavy Metal Toxicity, Int. J. Electrochem. 2011 (2011) 1-15.

[163] V. Dhull, A. Gahlaut, N. Dilbaghi, V. Hooda, Acetylcholinesterase biosensors for electrochemical detection of organophosphorus compounds: A review, Biochem. Res. Int. 2013 (2013). 
[164] C.S. Pundir, N. Chauhan, Acetylcholinesterase inhibition-based biosensors for pesticide determination: A review, Anal. Biochem. 429 (2012) 19-31.

[165] A. Attar, L. Cubillana-Aguilera, I. Naranjo-Rodríguez, J.L.H.H. de Cisneros, J.M. Palacios-Santander, A. Amine, Amperometric inhibition biosensors based on horseradish peroxidase and gold sononanoparticles immobilized onto different electrodes for cyanide measurements, Bioelectrochemistry. 101 (2015) 84-91.

[166] P.N. Nomngongo, J.C. Ngila, V.O. Nyamori, E.A. Songa, E.I. Iwuoha, Determination of selected heavy metals using amperometric horseradish peroxidase (HRP) inhibition biosensor, Anal. Lett. 44 (2011) 2031-2046.

[167] D. Shan, Q. Li, H. Xue, S. Cosnier, A highly reversible and sensitive tyrosinase inhibition-based amperometric biosensor for benzoic acid monitoring, Sensors Actuators B Chem. 134 (2008) 1016-1021.

[168] E. Asav, E. Yorganci, E. Akyilmaz, An inhibition type amperometric biosensor based on tyrosinase enzyme for fluoride determination, Talanta. 78 (2009) 553-556.

[169] L. Campanella, D. Lelo, E. Martini, M. Tomassetti, Organophosphorus and carbamate pesticide analysis using an inhibition tyrosinase organic phase enzyme sensor; comparison by butyrylcholinesterase+choline oxidase opee and application to natural waters, Anal. Chim. Acta. 587 (2007) 22-32.

[170] M.L. Shuler, F. Kargi, Enzymes, in: Bioprocess Eng. - Basic Concepts, 2nd ed., Upper Saddle River, NJ : Prentice Hall, 2002: pp. 57-97.

[171] F.W. Scheller, A. Yarman, T. Bachmann, Biosensors based on Aptamers and Enzymes, 1st ed., Springer International Publishing, 2014.

[172] R. Nenkova, D. Ivanova, J. Vladimirova, T. Godjevargova, New amperometric glucose biosensor based on cross-linking of glucose oxidase on silica gel/multiwalled 
carbon nanotubes/polyacrylonitrile nanocomposite film, Sensors Actuators, B Chem. 148 (2010) 59-65.

[173] A.L. Eckermann, D.J. Feld, J.A. Shaw, T.J. Meade, Electrochemistry of redoxactive self-assembled monolayers, Coord. Chem. Rev. 254 (2010) 1769-1802.

[174] Z. Xia, F. Leonardi, M. Gobbi, Y. Liu, V. Bellani, A. Liscio, A. Kovtun, R. Li, X. Feng, E. Orgiu, P. Samorì, E. Treossi, V. Palermo, Electrochemical Functionalization of Graphene at the Nanoscale with Self-Assembling Diazonium Salts, ACS Nano. 10 (2016) $7125-7134$.

[175] M. Vert, J. Chen, K.-H. Hellwich, P. Hodge, T. Nakano, C. Scholz, S. Slomkowski, J. Vohlidal, Recommendations and Terminology for Lactic acid-based Polymers, 2014.

[176] J.B. Ewaschuk, J.M. Naylor, G.A. Zello, D-Lactate in Human and Ruminant Metabolism, Am. Soc. Nutr. Sci. (2005) 1619-1625.

[177] B. Stallknecht, G.H. Vissing J, Lactate production and clearance in exercise. Effects of training. A mini-review, Scand. J. Med. Sci. Sports. 8 (1998) 127-131.

[178] L.B. Gladden, Lactate metabolism: A new paradigm for the third millennium, J Physiol. 558 (2004) 5-30.

[179] S. Cairns, Lactic Acid and Exercise Performance, Sport. Med. 36 (2006) 279-291.

[180] R.A. Robergs, F. Ghiasvand, D. Parker, Biochemistry of exercise-induced metabolic acidosis, Am. J. Physiol. Regul. Integr. Comp. Physiol. 287 (2004) R502-16.

[181] D. Cohen, Robert, H.F. Woods, Lactic Acidosis Revisited, Diabetes. 32 (1983) $181-191$.

[182] Y. Fujita, K. Kawaji, A. Kanamori, K. Matoba, Y. Yajima, A. Takeuchi, K. Ishii, Relationship between age-adjusted heart rate and anaerobic threshold in estimating exercise intensity in diabetics, Diabetes Res. Clin. Pract. 8 (1990) 69-74. 
[183] M.L. Goodwin, J.E. Harris, A. Hernández, L.B. Gladden, Blood lactate measurements and analysis during exercise: a guide for clinicians, J. Diabetes Sci. Technol. 1 (2007) 558-69.

[184] J. Bakker, P. Gris, M. Coffernils, R.J. Kahn, J.-L. Vincent, Serial blood lactate levels can predict the development of multiple organ failure following septic shock, Am. J. Surg. 171 (1996) 221-226.

[185] P.J. Derbyshire, H. Barr, F. Davis, S.P.J. Higson, Lactate in human sweat: A critical review of research to the present day, J. Physiol. Sci. 62 (2012) 429-440.

[186] N. Nikolaus, B. Strehlitz, Amperometric lactate biosensors and their application in (sports) medicine, for life quality and wellbeing, Microchim. Acta. 160 (2008) 15-55.

[187] S. Pérez, E. Fàbregas, Amperometric bienzymatic biosensor for L-lactate analysis in wine and beer samples, Analyst. 137 (2012) 3854.

[188] A. Lonvaud-Funel, Lactic acid bacteria in the quality improvement and depreciation of wine, Antonie van Leeuwenhoek, Int. J. Gen. Mol. Microbiol. 76 (1999) 317-331.

[189] S.B. Baker, W.H. Summerson, Colorimetric determination of lactic acid in biological material, J. Biol. Chem. 138 (1941) 535-554.

[190] I.S. Kucherenko, Ya.V. Topolnikova, O.O. Soldatkin, Advances in the biosensors for lactate and pyruvate detection for medical applications: a review, TrAC Trends Anal. Chem. 110 (2018) 160-172.

[191] L. Roque, I. Escudero, J.M. Benito, Lactic acid recovery by microfiltration using niosomes as extraction agents, Sep. Purif. Technol. 151 (2015) 1-13.

[192] M.P. Milagres, C. Cardoso Brandão Sebastião, M.A. Magalhães, V.P.R. Minim, L.A. Minim, Development and validation of the high performance liquid chromatography - ion exclusion method for detection of lactic acid in milk, Food Chem. 135 (2012) 10781082. 
[193] M. Numako, T. Toyo, I. Noge, Y. Kitagawa, H. Mizuno, Risk assessment of diabetes mellitus using dried saliva spot followed by ultra-performance liquid chromatography with fluorescence and mass spectrometry, Microchem. J. 142 (2018) 202207.

[194] C.-L. Hsieh, R. Koga, A. Furusho, T. Akita, M. Mita, T. Ide, J.-A. Lee, K. Hamase, Enantioselective and simultaneous determination of lactate and 3-hydroxybutyrate in human plasma and urine using a narrow-bore online two-dimensional high-performance liquid chromatography system, J. Sep. Sci. 41 (2018) 1298-1306.

[195] M. Peng, Y. Cai, X. Fang, L. Liu, Rapid quantification of metabolic intermediates in blood by liquid chromatography-tandem mass spectrometry to investigate congenital lactic acidosis, Anal. Chim. Acta. 942 (2016) 50-57.

[196] A. Zhou, J. Ni, Z. Xu, Y. Wang, H. Zhang, W. Wu, S. Lu, P.C. Karakousis, Y. Yao, Metabolomics specificity of tuberculosis plasma revealed by ${ }^{1} \mathrm{H}$ NMR spectroscopy, Tuberculosis. 95 (2015) 294-302.

[197] D.P. Soares, M. Law, Magnetic resonance spectroscopy of the brain: review of metabolites and clinical applications, Clin. Radiol. 64 (2009) 12-21.

[198] J. Ren, A.D. Sherry, C.R. Malloy, Noninvasive Monitoring of Lactate Dynamics in Human Forearm Muscle After Exhaustive Exercise by ${ }^{1} \mathrm{H}$-Magnetic Resonance Spectroscopy at 7 Tesla, Magn. Reson. Med. 70 (2013) 610-619.

[199] U. Sharma, D. Upadhyay, S. Mewar, A. Mishra, P. Das, S.D. Gupta, S.N. Dwivedi, G.K. Makharia, N.R. Jagannathan, Metabolic abnormalities of gastrointestinal mucosa in celiac disease: An in vitro proton nuclear magnetic resonance, J. Gastroenterol. Hepatol. 30 (2015) 1492-1498.

[200] F. Wu, Y. Huang, C. Huang, Chemiluminescence biosensor system for lactic acid using natural animal tissue as recognition element, Biosens. Bioelectron. 21 (2005) 518522. 
[201] T. Larsen, Fluorometric determination of D-lactate in biological fluids, Anal. Biochem. 539 (2017) 152-157.

[202] J. Galbán, S. De Marcos, J.R. Castillo, Fluorometric-Enzymatic Lactate Determination Based Enzyme Cytochrome b2 Fluorescence, Anal. Chem. 65 (1993) 30763080 .

[203] L.N. Borshchevskaya, T.L. Gordeeva, A.N. Kalinina, S.P. Sineokii, Spectrophotometric Determination of Lactic Acid, J. Anal. Chem. 71 (2016) 755-758.

[204] K. Rathee, V. Dhull, R. Dhull, S. Singh, Biosensors based on electrochemical lactate detection: A comprehensive review, Biochem. Biophys. Reports. 5 (2016) 35-54.

[205] C.S. Pundir, V. Narwal, B. Batra, Determination of lactic acid with special emphasis on biosensing methods: A review, Biosens. Bioelectron. 86 (2016) 777-790.

[206] L. Rassaei, W. Olthuis, S. Tsujimura, E.J.R. Sudhölter, A. Van Den Berg, Lactate biosensors: Current status and outlook, Anal. Bioanal. Chem. 406 (2014) 123-137.

[207] A. Gue, H. Tap, P. Gros, F. Maury, A miniaturised silicon based enzymatic biosensor: towards a generic structure and technology for multi-analytes assays, Sensors Actuators B. Chem. 82 (2002) 227-232.

[208] M.M. Rahman, M.J.A. Shiddiky, A. Rahman, Y. Shim, A lactate biosensor based on lactate dehydrogenase / nictotinamide adenine dinucleotide (oxidized form) immobilized on a conducting polymer / multiwall carbon nanotube composite film, Anal. Biochem. 384 (2009) 159-165.

[209] A.C. Pereira, M.R. Aguiar, A. Kisner, D. V Macedo, L.T. Kubota, Amperometric biosensor for lactate based on lactate dehydrogenase and Meldola Blue coimmobilized on multi-wall carbon-nanotube, Sensors Actuators B. Chem. 124 (2007) 269-276. 
[210] B. Batra, V. Narwal, C.S. Pundir, An amperometric lactate biosensor based on lactate dehydrogenase immobilized onto graphene oxide nanoparticles-modified pencil graphite electrode, Eng. Life Sci. 16 (2016) 786-794.

[211] B.K. Jena, C.R. Raj, Electrochemical Biosensor Based on Integrated Assembly of Dehydrogenase Enzymes and Gold Nanoparticles, Anal. Chem. 78 (2006) 6332-6339.

[212] M. Özkan, E. Erhan, Ö. Terzi, K. Özöner, Thermostable amperometric lactate biosensor with Clostridium thermocellum L-LDH for the measurement of blood lactate, Talanta. 79 (2009) 1412-1417.

[213] M. Piano, S. Serban, R. Pittson, G.A. Drago, J.P. Hart, Amperometric lactate biosensor for flow injection analysis based on a screen-printed carbon electrode containing Meldola' s Blue-Reinecke salt, coated with lactate dehydrogenase and $\mathrm{NAD}^{+}$, Talanta. 82 (2010) 34-37.

[214] H. Cheng, C. Hu, Z. Ji, W. Ma, H. Wang, A solid ionic Lactate biosensor using doped graphene-like membrane of Au-EVIMC-Titania nanotubes-polyaniline, Biosens. Bioelectron. 118 (2018) 97-101.

[215] D. Chan, M.M. Barsan, Y. Korpan, C.M.A. Brett, L-lactate selective impedimetric bienzymatic biosensor based on lactate dehydrogenase and pyruvate oxidase, Electrochim. Acta. 231 (2017) 209-215.

[216] Y. Umena, K. Yorita, T. Matsuoka, A. Kita, K. Fukui, Y. Morimoto, The crystal structure of L-lactate oxidase from Aerococcus viridans at $2.1 \AA$ resolution reveals the mechanism of strict substrate recognition, Biochem. Biophys. Res. Commun. 350 (2006) 249-256.

[217] I. Leiros, E. Wang, T. Rasmussen, E. Oksanen, H. Repo, S.B. Petersen, P. Heikinheimo, E. Hough, The $2.1 \AA$ structure of Aerococcus viridans L-lactate oxidase (LOX), Acta Crystallogr. Sect. F Struct. Biol. Cryst. Commun. 62 (2006) 1185-1190. 
[218] S. Ghisla, V. Massey, New flavins for old: artificial flavins as active site probes of flavoproteins., Biochem. J. 239 (1986) 1-12.

[219] I. Leiros, E. Wang, T. Rasmussen, E. Oksanen, H. Repo, S.B. Petersen, P. Heikinheimo, E. Hough, The crystal structure of lactate oxidase. (2006) https://www.rcsb.org/structure/2J6X (accessed February 22, 2019).

[220] R. Garjonyte, Y. Yigzaw, R. Meskys, A. Malinauskas, L. Gorton, Prussian Blueand lactate oxidase-based amperometric biosensor for lactic acid, Sensors Actuators, B Chem. 79 (2001) 33-38.

[221] W. Shi, X. Luo, Y. Cui, A tube-integrated painted biosensor for glucose and lactate, Sensors (Switzerland). 18 (2018).

[222] A. Uzunoglu, I. Ramirez, E. Andreasen, L.A. Stanciu, Layer by layer construction of ascorbate interference-free amperometric lactate biosensors with lactate oxidase, ascorbate oxidase, and ceria nanoparticles, Microchim. Acta. 183 (2016) 1667-1675.

[223] T. Shimomura, T. Sumiya, M. Ono, T. Ito, T.A. Hanaoka, Amperometric L-lactate biosensor based on screen-printed carbon electrode containing cobalt phthalocyanine, coated with lactate oxidase-mesoporous silica conjugate layer, Anal. Chim. Acta. 714 (2012) 114-120.

[224] M.C. Strumia, C.I. Alvarez Igarzabal, A.M. Baruzzi, F. Garay, M.R. Romero, D. Peralta, Supramolecular complex based on MWNTs/Boltorn H40 provides fast response to a Sandwich-type amperometric lactate biosensor, Sensors Actuators B Chem. 244 (2017) $577-584$.

[225] M.R. Romero, F. Garay, A.M. Baruzzi, Design and optimization of a lactate amperometric biosensor based on lactate oxidase cross-linked with polymeric matrixes, Sensors Actuators, B Chem. 131 (2008) 590-595. 
[226] R. Monošík, M. Stred'anský, G. Greif, E. Šturdík, A rapid method for determination of L-lactic acid in real samples by amperometric biosensor utilizing nanocomposite, Food Control. 23 (2012) 238-244.

[227] I. Bravo, M. Revenga-Parra, F. Pariente, E. Lorenzo, Reagent-less and robust biosensor for direct determination of lactate in food samples, Sensors (Switzerland). 17 (2017) 1-11.

[228] A. Parra, E. Casero, L. Vázquez, F. Pariente, E. Lorenzo, Design and characterization of a lactate biosensor based on immobilized lactate oxidase onto gold surfaces, Anal. Chim. Acta. 555 (2006) 308-315.

[229] L. V. Shkotova, T.B. Goriushkina, C. Tran-Minh, J.M. Chovelon, A.P. Soldatkin, S. V. Dzyadevych, Amperometric biosensor for lactate analysis in wine and must during fermentation, Mater. Sci. Eng. C. 28 (2008) 943-948.

[230] L. V. Shkotova, N.Y. Piechniakova, O.L. Kukla, S. V. Dzyadevych, Thin-film amperometric multibiosensor for simultaneous determination of lactate and glucose in wine, Food Chem. 197 (2016) 972-978.

[231] P. Giménez-Gómez, M. Gutiérrez-Capitán, F. Capdevila, A. Puig-Pujol, C. Fernández-Sánchez, C. Jiménez-Jorquera, Monitoring of malolactic fermentation in wine using an electrochemical bienzymatic biosensor for L-lactate with long term stability, Anal. Chim. Acta. 905 (2016) 126-133.

[232] D. Jiang, Z. Chu, J. Peng, W. Jin, Screen-printed biosensor chips with Prussian blue nanocubes for the detection of physiological analytes, Sensors Actuators, B Chem. 228 (2016) 679-687.

[233] S.B. Hall, E.A. Khudaish, A.L. Hart, Electrochemical oxidation of hydrogen peroxide at platinum electrodes. Part V: inhibition by chloride, Electrochim. Acta. 45 (2000) 3573-3579. 
[234] Ekfdiagnostics, Lactate Scout 4 - Lactate Analyzer for athletes, (n.d.) 4. https://www.ekfdiagnostics.com/lactate-scout.html (accessed February 22, 2019).

[235] Novabio, Lactate Plus, (n.d.). www.novabio.us/lactate-plus/ (accessed February 22, 2019).

[236] Novabio, StatStrip Lac, (n.d.). http:www.novabio.us/statstrip-lactate/ (accessed February 22, 2019).

[237] Apexbio Taiwian, THE https://www.apexbio.com/Web/Products/content.php?product=AS60000F3 （accessed February 22, 2019).

[238] Phill Bates Sports Promotions Pty Ltd, Lactate Pro TM - Lactate Analyzer, (n.d.). http://www.lactatepro.com.au/lactatepro/ (accessed February 22, 2019).

[239] ekfdiagnostics, Biosen C-Line - Glucose and Lactate analyzers, (n.d.). https://www.ekfdiagnostics.com/cgi-bin/showpage.fc (accessed February 22, 2019).

[240] P.J. Lamas-Ardisana, O.A. Loaiza, L. Añorga, E. Jubete, M. Borghei, V. Ruiz, E. Ochoteco, G. Cabañero, H.J. Grande, Disposable amperometric biosensor based on lactate oxidase immobilised on platinum nanoparticle-decorated carbon nanofiber and poly(diallyldimethylammonium chloride) films, Biosens. Bioelectron. 56 (2014) 345-351.

[241] G.G. Guilbault, G. Palleschi, G. Lubrano, Non-invasive biosensors in clinical analysis, Biosens. Bioelectron. 10 (1995) 379-392.

[242] E. Tékus, M. Kaj, E. Szabó, N.L. Szénási, I. Kerepesi, M. Figler, R. Gábriel, M. Wilhelm, Comparison of blood and saliva lactate level after maximum intensity exercise, Acta Biol. Hung. 63 Suppl 1 (2012) 89-98.

[243] R. Segura, C. Javierre, J.L. Ventura, M.A. Lizarraga, B. Campos, E. Garrido, A new approach to the assessment of anaerobic metabolism: measurement of lactate in saliva, $\mathrm{Br}$. J. Sports Med. 30 (1996) 305-309. 
[244] A. Aguirre, J.A. Banderas, G.G. Haraszthy, M.S. Reddy, M.J. Levine, Sialochemistry: A Diagnostic Tool?, Crit. Rev. Oral Biol. Med. 4 (1993) 343-350.

[245] K. Petropoulos, S. Piermarini, S. Bernardini, G. Palleschi, D. Moscone, Development of a disposable biosensor for lactate monitoring in saliva, Sensors Actuators, B Chem. 237 (2016) 8-15.

[246] A.M. Spehar-Délèze, S. Anastasova, P. Vadgama, Electropolymerised Phenolic Films as Internal Barriers for Oxidase Enzyme Biosensors, Electroanalysis. 26 (2014) $1335-1344$.

[247] J. Ballesta Claver, M.C. Valencia Mirón, L.F. Capitán-Vallvey, Disposable electrochemiluminescent biosensor for lactate determination in saliva, Analyst. 134 (2009) $1423-32$.

[248] M.H. Faridnia, G. Palleschi, G.J. Lubrano, G.G. Guilbault, Amperometric biosensor for determination of lactate in sweat, Anal. Chim. Acta. 278 (1993) 35-40.

[249] M.J. Buono, N.V.L. Lee, P.W. Miller, The relationship between exercise intensity and the sweat lactate excretion rate, J. Physiol. Sci. 60 (2010) 103-107.

[250] P. Pilardeau, J. Vaysse, M. Garnier, M. Joublin, L. Valeri, Secretion of eccrine sweat glands during exercise, Br. J. Sports Med. 13 (1979) 118-21.

[251] S. Wolfe, G. Cage, M. Epstein, L. Tice, H. Miller, R.S. Gordon, Metabolic studies of isolated human eccrine sweat glands, J. Clin. Invest. 49 (1970) 1880-1884.

[252] J.M. Green, R.C. Pritchett, T.R. Crews, J.R. McLester, D.C. Tucker, Sweat lactate response between males with high and low aerobic fitness, Eur. J. Appl. Physiol. 91 (2004) $1-6$.

[253] A.B. Stefaniak, C.J. Harvey, Dissolution of materials in artificial skin surface film liquids, Toxicol. Vitr. 20 (2006) 1265-1283. 
[254] C.J. Harvey, R.F. LeBouf, A.B. Stefaniak, Formulation and stability of a novel artificial sebum under conditions of storage and use, Int. J. Cosmet. Sci. 32 (2010) 347355.

[255] S.M. Koch, R. W. Taylor, Chloride ion in intensive care medicine, Crit. Care Med. 20 (1992) 227-240.

[256] A.C. Galvis-Sánchez, I. V. Tóth, A. Portela, I. Delgadillo, A.O.S.S. Rangel, Monitoring sodium chloride during cod fish desalting process by flow injection spectrometry and infrared spectroscopy, Food Control. 22 (2011) 277-282.

[257] R. Pérez-Olmos, R. Herrero, J.L.F.C. Lima, M.C.B.S.M. Montenegro, Sequential potentiometric determination of chloride and nitrate in meat products, Food Chem. 59 (1997) 305-311.

[258] J.A. Hern, G.K. Rutherford, G.W. Vanloon, Determination of chloride, nitrate, sulphate and total sulphur in environmental samples by single-column ion chromatography, Talanta. 30 (1983) 677-682.

[259] F.J. Millero, R. Feistel, D.G. Wright, T.J. McDougall, The composition of Standard Seawater and the definition of the Reference-Composition Salinity Scale, Deep. Res. Part I Oceanogr. Res. Pap. 55 (2008) 50-72.

[260] J.A. Morales, L.S. De Graterol, J. Mesa, Determination of chloride, sulfate and nitrate in groundwater samples by ion chromatography, J. Chromatogr. A. 884 (2000) 185190.

[261] A. Cranny, N. Harris, N. White, Screen printed potentiometric chloride sensors, Procedia Eng. 87 (2014) 220-223.

[262] WHO, Reducing salt intake in populations, 2007. 
[263] J.P. Dzoyem, V. Kuete, J.N. Eloff, Biochemical Parameters in Toxicological Studies in Africa: Significance, Principle of Methods, Data Interpretation, and Use in Plant Screenings, Elsevier Inc., 2014.

[264] W.P. Mutter, C.A. Korzelius, Urine Chemistries, Hosp. Med. Clin. 1 (2012) e338e352.

[265] R.W. Schrier, Diagnostic Value of Urinary Sodium, Chloride, Urea, and Flow, J. Am. Soc. Nephrol. 22 (2011) 1610-1613.

[266] G.T. Nagami, Hyperchloremia - Why and how, Nefrologia. 36 (2016) 347-353.

[267] G. Morrison, Serum Chloride., in: H.K. Walker, W.D. Hall, J.W. Hurst (Eds.), Clin. Methods Hist. Phys. Lab. Exam., 3rd ed., Boston: Butterworths, 1990: pp. 890-894.

[268] S. Watanabe, T. Kimura, K. Suenaga, S. Wada, K. Tsuda, S. Kasama, T. Takaoka, K. Kajiyama, M. Takeda, H. Yoshikawa, Decreased chloride levels of cerebrospinal fluid in patients with amyotrophic lateral sclerosis, J. Neurol. Sci. 285 (2009) 146-148.

[269] W.B. Guggino, B.A. Stanton, New insights into cystic fibrosis: Molecular switches that regulate CFTR, Nat. Rev. Mol. Cell Biol. 7 (2006) 426-436.

[270] P.M. Farrell, T.B. White, N. Derichs, C. Castellani, B.J. Rosenstein, Cystic Fibrosis Diagnostic Challenges over 4 Decades: Historical Perspectives and Lessons Learned, J. Pediatr. 181 (2017) S16-S26.

[271] P.R. Sosnay, T.B. White, P.M. Farrell, C.L. Ren, N. Derichs, M.S. Howenstine, J.A. Nick, K. De Boeck, Diagnosis of Cystic Fibrosis in Nonscreened Populations, J. Pediatr. 181 (2017) S52-S57.

[272] M.I. Barrio Gómez de Agüero, G. García Hernández, S. Gartner, Protocolo de diagnóstico y seguimiento de los pacientes con fibrosis quística, An. Españoles Pediatr. 50 (1999) 625-634. 
[273] M.D. Robert C. Stern, The diagnosis of cystic fibrosis, N. Engl. J. Med. 336 (1997) $487-491$.

[274] B.J. Rosenstein, G.R. Cutting, The diagnosis of cystic fibrosis: A consensus statement, J. Pediatr. 132 (1998) 589-595.

[275] K. Sato, W.H. Kang, K. Saga, K.T. Sato, Biology of sweat glands and their disorders. I. Normal sweat gland function, J. Am. Acad. Dermatol. 20 (1989) 537-563.

[276] A. Mena-Bravo, M.D. Luque de Castro, Sweat: A sample with limited present applications and promising future in metabolomics, J. Pharm. Biomed. Anal. 90 (2014) $139-147$.

[277] V.A. LeGrys, J.R. Yankaskas, L.M. Quittell, B.C. Marshall, P.J. Mogayzel, Diagnostic Sweat Testing: The Cystic Fibrosis Foundation Guidelines, J. Pediatr. 151 (2007) 85-89.

[278] W.A. Latzka, S.J. Montain, Water and electrolyte requirements for exercise. Clin. Sports Med. 18 (1999) 513-524.

[279] H.W. Doughty, Mohr's method for the determination of silver and halogens in other than neutral solutions, J. Am. Chem. Soc. 46 (1924) 2707-2709.

[280] J.R. Caldwell, H. V. Moyer, Determination of Chloride: A Modification of the Volhard Method, Ind. Eng. Chem. - Anal. Ed. 7 (1935) 38-39.

[281] M.N. Haouet, M.S. Altissimi, M. Framboas, R. Galarini, Validation of the Volhard method for chloride determination in food, Accredit. Qual. Assur. 11 (2006) 23-28.

[282] E.B. Silva, F.D. Nogueira, P.T.G. Guimarães, M.R. Malta, Chloride analysis methods and contents in leaves, grains, and husks of coffee, Commun. Soil Sci. Plant Anal. 29 (1998) 2319-2331. 
[283] J.S.F. Pereira, L.O. Diehl, F.A. Duarte, M.F.P. Santos, R.C.L. Guimarães, V.L. Dressler, É.M.M. Flores, Chloride determination by ion chromatography in petroleum coke after digestion by microwave-induced combustion, J. Chromatogr. A. 1213 (2008) 249252.

[284] A.M. Pimenta, A.N. Araújo, M.C.B.S.M. Montenegro, C. Pasquini, J.J.R. Rohwedder, I.M. Raimundo, Chloride-selective membrane electrodes and optodes based on an indium(III) porphyrin for the determination of chloride in a sequential injection analysis system, J. Pharm. Biomed. Anal. 36 (2004) 49-55.

[285] H. Cao, D.H. Wu, Rapid and sensitive determination of trace chloride ion in drinks using resonance light scattering technique, J. Autom. Methods Manag. Chem. 2008 (2008) $0-5$.

[286] R.B. Mesquita, S.M. Fernandes, A.O. Rangel, Turbidimetric determination of chloride in different types of water using a single sequential injection analysis system, $\mathbf{J}$ Env. Monit. 4 (2002) 458-461.

[287] M.O. Gorbunova, A.V. Shevchenko, V.V. Apyari, A.A. Furletov, P.A. Volkov, A.V. Garshev, S.G. Dmitrienko, Selective determination of chloride ions using silver triangular nanoplates and dynamic gas extraction, Sensors Actuators, B Chem. 256 (2018) 699-705.

[288] A. Koh, D. Kang, Y. Xue, S. Lee, R.M. Pielak, J. Kim, T. Hwang, S. Min, A. Banks, M.C. Manco, L. Wang, K.R. Ammann, K. Jang, S. Han, R. Ghaffari, U. Paik, M.J. Slepian, Y. Huang, J.A. Rogers, A Soft, Wearable Microfluidic Device for the Capture, Storage, and Colorimetric Sensing of Sweat, Sci Transl Med. 8 (2017).

[289] A. Martin, R. Narayanaswamy, Studies on quenching of fluorescence of reagents in aqueous solution leading to an optical chloride-ion sensor, Sensors Actuators B Chem. 39 (1997) 330-333. 
[290] V.G. Bonifácio, L.C. Figueiredo-Filho, L.H. Marcolino, O. Fatibello-Filho, An improved flow system for chloride determination in natural waters exploiting solid-phase reactor and long pathlength spectrophotometry, Talanta. 72 (2007) 663-667.

[291] L.S. Laxmeshwar, M.S. Jadhav, J.F. Akki, P. Raikar, J. Kumar, O. prakash, R. Mahakud, U.S. Raikar, Quantification of chloride and iron in sugar factory effluent using long period fiber grating chemical sensor, Sensors Actuators B Chem. 258 (2018) 850-856.

[292] Y. Zhu, R. Zhang, J. Luo, D. Tang, S. Xu, X. Liu, One-pot facile preparation of Ag nanoparticles for chloride ion sensing, Colloid Polym. Sci. 294 (2016) 1643-1649.

[293] ELITechGroup - Biomedical Systems, Macroduct@/Sweat.ChekTM - Sweat testing system, ELITechGroup - Biomedical Systems, 2014. (https://www.elitechgroup.com/wpcontent/uploads/2015/01/macroduct_20141.pdf.)

[294] P. Díaz, Z. González, M. Granda, R. Menéndez, R. Santamaría, C. Blanco, Evaluating capacitive deionization for water desalination by direct determination of chloride ions, Desalination. 344 (2014) 396-401.

[295] R.G. Du, R.G. Hu, R.S. Huang, C.J. Lin, In situ measurement of Cl- concentrations and $\mathrm{pH}$ at the reinforcing steel/concrete interface by combination sensors, Anal. Chem. 78 (2006) 3179-3185.

[296] W.T. Grubb, Chloride - selective electrode, US3740326, 1973.

[297] A. Hulanicki, A. Michalska, All-solid-state chloride-selective electrode with poly(pyrrole) solid contact, Electroanalysis. 7 (1995) 692-693.

[298] D.H. Choi, Y. Li, G.R. Cutting, P.C. Searson, A wearable potentiometric sensor with integrated salt bridge for sweat chloride measurement, Sensors Actuators, B Chem. 250 (2017) 673-678.

[299] D.B. de Graaf, Y. Abbas, J.G. Bomer, W. Olthuis, A. van den Berg, Sensor-actuator system for dynamic chloride ion determination, Anal. Chim. Acta. 888 (2015) 44-51. 
[300] J. Bujes-Garrido, M.J. Arcos-Martínez, Disposable sensor for electrochemical determination of chloride ions, Talanta. 155 (2016) 153-157.

[301] V.A.T. Dam, M.A.G. Zevenbergen, R. Van Schaijk, Flexible chloride sensor for sweat analysis, Procedia Eng. 120 (2015) 237-240.

[302] I. Campos, R. Masot, M. Alcañiz, L. Gil, J. Soto, J.L. Vivancos, E. García-Breijo, R.H. Labrador, J.M. Barat, R. Martínez-Mañez., Accurate concentration determination of anions nitrate, nitrite and chloride in minced meat using a voltammetric electronic tongue, Sensors Actuators, B Chem. 149 (2010) 71-78.

[303] A.J. Bandodkar, J. Wang, Non-invasive wearable electrochemical sensors: A review, Trends Biotechnol. 32 (2014) 363-371.

[304] M. Bariya, H.Y.Y. Nyein, A. Javey, Wearable sweat sensors, Nat. Electron. 1 (2018) 160-171.

[305] H.S. Toh, C. Batchelor-Mcauley, K. Tschulik, R.G. Compton, Electrochemical detection of chloride levels in sweat using silver nanoparticles: A basis for the preliminary screening for cystic fibrosis, Analyst. 138 (2013) 4292-4297.

[306] A.T. Kuhn, P.M. Wright, The behaviour of platinum, iridium and ruthenium electrodes in strong chloride solutions, J. Electroanal. Chem. 41 (1973) 329-349.

[307] E.L. Littauer, L.L. Shreir, Anodic polarization of platinum in sodium chloride solutions, Electrochim. Acta. 11 (1966) 527-536.

[308] J.A. Polta, D.C. Johnson, Pulsed Amperometric Detection of Electroinactive Adsorbates at Platinum Electrodes in a Flow Injection System, Anal. Chem. 57 (1985) $1373-1376$.

[309] N. Priyantha, S. Malavipathirana, Effect of chloride irons on the electrochemical behaviour of platinum surfaces, J. Natl. Sci. Found. Sri Lanka. 24 (1996) 237-246. 
[310] J. Clavilier, The role of anion on the electrochemical behaviour of a $\{111\}$ platinum surface; an unusual splitting of the voltammogram in the hydrogen region, J. Electroanal. Chem. 107 (1980) 211-216.

[311] J. Gulens, Surface effects in relation to the response of solid-state ion-selective eletrodes, Ion-Selective Electrode Rev. 2 (1981) 117-157.

[312] S.N. Davis, D.O. Whittemore, J. Fabryka-Martin, Uses of chloride/bromide ratios in studies of potable water, Ground Water. 36 (1998) 338-350.

[313] A.S. McCall, C.F. Cummings, G. Bhave, R. Vanacore, A. Page-Mccaw, B.G. Hudson, Bromine is an essential trace element for assembly of collagen IV scaffolds in tissue development and architecture, Cell. 157 (2014) 1380-1392.

[314] R. Sosa, W.J. Stone, Bromide toxicity from consumption of dead sea salt, Am. J. Med. 123 (2010) e11-e12.

[315] G.J. Bowers, M. Onoroski, Hyperchloremia and the incidence of Bromism in 1990, Clin. Chem. 36 (1990) 1399-1403.

[316] T.A. Ban, Pharmacotherapy of mental illness - A historical analysis, Prog. NeuroPsychopharmacology Biol. Psychiatry. 25 (2001) 709-727.

[317] D. Vlascici, N. Plesu, G. Fagadar-Cosma, A. Lascu, M. Petric, M. Crisan, A. Belean, E. Fagadar-Cosma, Potentiometric sensors for iodide and bromide based on Pt(II)porphyrin, Sensors (Switzerland). 18 (2018).

[318] T. Quast, M. Combs, S. Edwards, Pharmacokinetics of bromide in adult sheep following oral and intravenous administration, Aust. Vet. J. 93 (2015) 20-25.

[319] B. Gindiciosi, V. Palus, S. Eminaga, E. Villiers, G. Bruto Cherubini, Serum bromide concentrations following loading dose in epileptic dogs, J. Small Anim. Pract. 55 (2014) $108-111$. 
[320] E. Ito, K.W. Yip, D. Katz, S.B. Fonseca, D.W. Hedley, S. Chow, G.W. Xu, T.E. Wood, C. Bastianutto, A.D. Schimmer, S.O. Kelley, Potential use of cetrimonium bromide as an apoptosis-promoting anticancer agent for head and neck cancer, Mol. Pharmacol. 76 (2009) 969-983.

[321] L. Fu, C. Li, Y. Li, S. Chen, Y. Long, R. Zeng, Simultaneous determination of iodide and bromide using a novel LSPR fluorescent Ag nanocluster probe, Sensors Actuators, B Chem. 240 (2017) 315-321.

[322] T.P. Bonacquisti, A drinking water utility's perspective on bromide, bromate, and ozonation, Toxicology. 221 (2006) 145-148.

[323] M. Sun, G. V. Lowry, K.B. Gregory, Selective oxidation of bromide in wastewater brines from hydraulic fracturing, Water Res. 47 (2013) 3723-3731.

[324] K.B. Gregory, R.D. Vidic, D.A. Dzombak, Water management challenges associated with the production of shale gas by hydraulic fracturing, Elements. 7 (2011) $181-186$.

[325] K. Danchana, F. Maya, P. Wilairat, K. Uraisin, V. Cerdà, Spectrophotometric determination of bromide in water using the multisyringe flow injection analysis technique coupled to a gas-diffusion unit, Anal. Methods. 7 (2015) 4202-4208.

[326] S.D. Richardson, S.Y. Kimura, Water Analysis: Emerging Contaminants and Current Issues, Anal. Chem. 88 (2016) 546-582.

[327] S. Allard, J. Tan, C.A. Joll, U. Von Gunten, Mechanistic Study on the Formation of $\mathrm{Cl}^{-} / \mathrm{Br}^{-} / \mathrm{I}^{-}$Trihalomethanes during Chlorination/Chloramination Combined with a Theoretical Cytotoxicity Evaluation, Environ. Sci. Technol. 49 (2015) 11105-11114.

[328] Y.I. Maznaya, O. V. Zuy, V. V. Goncharuk, Monitoring of Drinking Waters for the Content of Bromide, Iodide, Bromate and Iodate Ions, J. Water Chem. Technol. 40 (2019) $379-385$. 
[329] J. Criquet, E.M. Rodriguez, S. Allard, S. Wellauer, E. Salhi, C.A. Joll, U. von Gunten, Reaction of bromine and chlorine with phenolic compounds and natural organic matter extracts - Electrophilic aromatic substitution and oxidation, Water Res. 85 (2015) $476-486$.

[330] M.R. Flórez, M. Resano, Direct determination of bromine in plastic materials by means of solid sampling high-resolution continuum source graphite furnace molecular absorption spectrometry, Spectrochim. Acta - Part B At. Spectrosc. 88 (2013) 32-39.

[331] A. García-Figueroa, F. Pena-Pereira, I. Lavilla, C. Bendicho, Headspace singledrop microextraction coupled with microvolume fluorospectrometry for highly sensitive determination of bromide, Talanta. 170 (2017) 9-14.

[332] L.S. Clescer, E. Greenberg, Arnold, A.D. Eaton, Standard methods for the examination of water and wastewater, 21st ed., Centennial Edition, 2005.

[333] K. Uraisin, T. Takayanagi, M. Oshima, D. Nacapricha, S. Motomizu, Kineticspectrophotometric method for the determination of trace amounts of bromide in seawater, Talanta. 68 (2006) 951-956.

[334] G. Hunter, Bromide Determination in Body Fluids, Biochem J. 54 (1952) 42-45.

[335] F. Zitomer, L.J. L., Spectrophotometric Determination of Bromide Ion in Water, Anal. Chem. 35 (1963) 146-149.

[336] H. Staerk, A. Suess, Bromine Content of Vegetables and its Accumulation After soil Fumigation with Methyl Bromide, Usind Neuron Activation Analysis, in: Comp. Stud. Food Environ. Contam., 1974: pp. 417-427.

[337] D.L. Rocha, M.C. Machado, W.R. Melchert, A sensitive flow-based procedure for spectrophotometric speciation analysis of inorganic bromine in waters, Talanta. 129 (2014) 93-99. 
[338] D.J. Zhang, Y. Cai, M.L. Chen, Y.L. Yu, J.H. Wang, Dielectric barrier dischargeoptical emission spectrometry for the simultaneous determination of halogens, J. Anal. At. Spectrom. 31 (2016) 398-405.

[339] H.F. Dobolyi, Field Determination of Bromide in Water, Anal. Chem. 56 (1984) 2961-2963.

[340] V. V. Goncharuk, O. V. Zuy, Y.I. Maznaya, A. V. Terletskaya, M. V. Milyukin, Determination of Mass Concentration of Bromide, Iodide and Nitrate Ions in Water, J. Water Chem. Technol. 40 (2018) 51-56.

[341] L.J. Loh, G.C. Bandara, G.L. Weber, V.T. Remcho, Detection of water contamination from hydraulic fracturing wastewater: $\mathrm{A} \mu \mathrm{pAD}$ for bromide analysis in natural waters, Analyst. 140 (2015) 5501-5507.

[342] M.E. Miller, J.M. Cosgriff, G.B. Forbes, Bromide space determination using anionexchange chromatography for measurement of bromide, Am. J. Clin. Nutr. 50 (1989) 168171.

[343] M. Neal, C. Neal, H. Wickham, S. Harman, Determination of bromide, chloride, fluoride, nitrate and sulphate by ion chromatography: Comparisons of methodologies for rainfall, cloud water and river waters at the Plynlimon catchments of mid-Wales, Hydrol. Earth Syst. Sci. 11 (2007) 294-300.

[344] R. García Fernández, J.I. García Alonso, A. Sanz-Medel, Coupling of ICP-MS with ion chromatography after conductivity suppression for the determination of anions in natural and waste waters, J. Anal. At. Spectrom. 16 (2001) 1035-1039.

[345] R.E. Sturgeon, Detection of bromine by ICP- oa -ToF-MS following photochemical vapor generation, Anal. Chem. 87 (2015) 3072-3079. 
[346] J.L. Vilchez, E. Manzano, R. Avidad, I. Orbe, L.F. Capitán-Vallvey, Spectrofluorimetric determination of traces of bromide, Mikrochim. Acta. 113 (1994) 2936.

[347] H. Gallardo, I. Queralt, J. Tapias, L. Candela, E. Margui, Bromine and bromide content in soils: Analytical approach from total reflection X-ray fluorescence spectrometry, Chemosphere. 156 (2016) 294-301.

[348] D. Midgley, A Bromide-Selective Electrode-Redox Electrode Cell for the Potentiometric Determination of Bromine and Free Residual Chlorine, Talanta. 30 (1983) $547-554$.

[349] A.M. Gerakis, M.A. Koupparis, Studies of Cetyltrimethyl-Ammonium Bromide Micellar System Using a Bromide Selective Electrode, Talanta. 41 (1994) 765-773.

[350] J.F. Van Staden, Flow Injection Determination of Inorganic Bromide in Soils with a Coated Tubular Solid-state Bromide-selective Electrode, Analyst. 112 (1987) 595-599.

[351] G. Colovos, G.S. Wilson, J.L. Moyers, Simultaneous Determination of Bromide and Chloride by Cathodic Stripping Voltammetry, Anal. Chem. 46 (1974) 1051-1054.

[352] J.J. Mutic, S.D. Nikolic-Mandic, A.D. Lolic, D.D. Manojlovic, Determination of iodide and bromide by flow methods with amperometric detection, J. Anal. Chem. 63 (2008) 907-913.

[353] S.D. Pretty, A.Y. Musa, J.C. Wren, Reactions of Bromide and Iodide Ions with Silver Oxide Films on Ag Substrates, J. Electrochem. Soc. 160 (2012) H13-H21.

[354] B. Bennett, J. Chang, A.J. Bard, Mechanism of the $\mathrm{Br}^{-} / \mathrm{Br}_{2}$ Redox Reaction on Platinum and Glassy Carbon Electrodes in Nitrobenzene by Cyclic Voltammetry, Electrochim. Acta. 219 (2016) 1-9.

[355] B. de Benoist, M. Andersson, I. Egli, B. Takkouche, H. Allen, Iodine status worldwide, 2004. 
[356] M.B. Zimmermann, Iodine deficiency, Endocr. Rev. 30 (2009) 376-408.

[357] WHO, Assessment of the iodine deficiency disorders and monitoring their elimination, 2007.

[358] J.E. Dumont, A.M. Ermans, C. Maenhaut, F. Coppée, J.B. Stanbury, Large goitre as a maladaptation to iodine deficiency, Clin. Endocrinol. (Oxf). 43 (1995) 1-10.

[359] F. Vermiglio, V.P. Lo Presti, M. Moleti, M. Sidoti, G. Tortorella, G. Scaffidi, M.G. Castagna, F. Mattina, M.A. Violi, A. Crisà, A. Artemisia, F. Trimarchi, Attention deficit and hyperactivity disorders in the offspring of mothers exposed to mild-moderate iodine deficiency: A possible novel iodine deficiency disorder in developed countries, J. Clin. Endocrinol. Metab. 89 (2004) 6054-6060.

[360] H.X. Weng, C.L. Hong, T.H. Xia, L.T. Bao, H.P. Liu, D.W. Li, Iodine biofortification of vegetable plants - An innovative method for iodine supplementation, Chinese Sci. Bull. 58 (2013) 2066-2072.

[361] M. Haldimann, A. Alt, A. Blanc, K. Blondeau, Iodine content of food groups, J. Food Compos. Anal. 18 (2005) 461-471.

[362] M. Haap, H.J. Roth, T. Huber, H. Dittmann, R. Wahl, Urinary iodine: Comparison of a simple method for its determination in microplates with measurement by inductivelycoupled plasma mass spectrometry, Sci. Rep. 7 (2017) 1-10.

[363] O. Chailapakul, M. Amatatongchai, P. Wilairat, K. Grudpan, D. Nacapricha, Flowinjection determination of iodide ion in nuclear emergency tablets, using boron-doped diamond thin film electrode, Talanta. 64 (2004) 1253-1258.

[364] J. Mihailović, Current concepts of 131I therapy in oncology: Indications, methods and follow up, Arch. Oncol. 14 (2006) 45-51.

[365] C. Durante, N. Haddy, E. Baudin, S. Leboulleux, D. Hartl, J.P. Travagli, B. Caillou, M. Ricard, J.D. Lumbroso, F. De Vathaire, M. Schlumberger, Long-term outcome of 444 
patients with distant metastases from papillary and follicular thyroid carcinoma: Benefits and limits of radioiodine therapy, J. Clin. Endocrinol. Metab. 91 (2006) 2892-2899.

[366] E. Hindie, P. Zanotti-Fregonara, I. Keller, F. Duron, J.-Y. Devaux, M. CalzadaNocaudie, E. Sarfati, J.-L. Moretti, P. Bouchard, M.-E. Toubert, Bone metastases of differentiated thyroid cancer: impact of early ${ }^{131}$ I-based detection on outcome, Endocr. Relat. Cancer. 14 (2007) 799-807.

[367] E.C. Nijssen, P.J. Nelemans, R.J. Rennenberg, V. Van Ommen, J.E. Wildberger, Evaluation of Safety Guidelines on the Use of Iodinated Contrast Material: Conundrum Continued, Invest. Radiol. 53 (2018) 616-622.

[368] C.S. Ng, S.P. Kalva, C. Gunnarsson, M.P. Ryan, E.R. Baker, R.L. Mehta, Risk of renal events following intravenous iodinated contrast material administration among inpatients admitted with cancer a retrospective hospital claims analysis, Cancer Imaging. 18 (2018) 1-14.

[369] C.P. Shelor, P.K. Dasgupta, Review of analytical methods for the quantification of iodine in complex matrices, Anal. Chim. Acta. 702 (2011) 16-36.

[370] B. Michalke, P. Schramel, Iodine speciation in biological samples by capillary electrophoresis-inductively coupled plasma mass spectrometry., Electrophoresis. 20 (1999) 2547-2553.

[371] E.B. Sandell, I.M. Kolthoff, Micro determination of iodine by a catalytic method, Mikrochim. Acta. 1 (1937) 9-25.

[372] M.C. Yebra, M.H. Bollaín, A simple indirect automatic method to determine total iodine in milk products by flame atomic absorption spectrometry, Talanta. 82 (2010) $828-$ 833.

[373] E. Vtorushina, I. Saprykin, G. Knapp, Optimization of the conditions of oxidation vapor generation for determining chlorine, bromine, and iodine in aqueous solutions by 
inductively coupled plasma atomic-emission spectrometry, J. Anal. Chem. 63 (2008) 643648.

[374] K. Isaac-Olive, T.T. Kyaw, A. Chatt, Estimation of anthropogenic organo-chlorine, bromine and iodine compounds in apolar lipid fractions of bovine milk by solid-phase extraction and neutron activation analysis (SPE-NAA), J. Radioanal. Nucl. Chem. 8 (2018).

[375] L. Rong, L.W. Lim, T. Takeuchi, Rapid determination of iodide in seawater samples by ion chromatography with chemically-bonded vitamin-U stationary phase, Microchem. J. 108 (2013) 113-116.

[376] P. Das, M. Gupta, A. Jain, K.K. Verma, Single drop microextraction or solid phase microextraction-gas chromatography-mass spectrometry for the determination of iodine in pharmaceuticals, iodized salt, milk powder and vegetables involving conversion into 4iodo-N,N-dimethylaniline, J. Chromatogr. A. 1023 (2004) 33-39.

[377] T. Tomiyasu, M. Nonaka, M. Uchikado, K. Anazawa, H. Sakamoto, Kinetic determination of total iodine in urine and foodstuffs using a mixed acid as a pretreatment agent., Anal. Sci. 20 (2004) 391-3.

[378] E.A. Crecelius, Determination of Total Iodine in Milk by X-Ray Fluorescence Spectrometry and Iodide Electrode, Anal. Chem. 47 (1975) 2034-2035.

[379] P. Ünak, Ş. Darcan, F. Yurt, Z. Biber, M. Çoker, Determination of iodide amounts in urine and water by isotope dilution analysis, Biol. Trace Elem. Res. 71-72 (1999) 463470.

[380] S.-Z. Yao, P. Chen, W.-Z. Wei, A quartz crystal microbalance method for the determination of iodine in foodstuffs, Food Chem. 67 (1999) 311-316.

[381] W. Zhang, A. Mnatsakanov, R. Hower, H. Cantor, Y. Wang, Urinary iodine assays and ionophore based potentiometric iodide sensors, Front. Biosci. 10 (2005) 88-93. 
[382] J. Melichercik, L. Szijarto, A R. Hill, Comparison of ion-specific electrode and high performance liquid chromatography methods for the determination of iodide in milk., J. Dairy Sci. 89 (2006) 934-7.

[383] F.C. de Souza, C.G. Vegas, D.A.I. da Silva, M.S. Ribeiro, M.F. Cabral, M.A. de Melo, R.M.T. Mattos, R.B. Faria, E. D'Elia, Amperometric and potentiometric determination of iodide using carbon electrodes modified with salophen complex, J. Electroanal. Chem. 783 (2016) 49-55.

[384] L. Yang, L. Zou, G. Li, B. Ye, Simple and rapid determination of trace iodide by cathodic stripping voltammetry, Talanta. 147 (2016) 634-640.

[385] K. Nakagawa, K. Suzuki, M. Kondo, S. Hayakawa, M. Nakayama, Electrosynthesis of Layered Organo-Manganese Dioxide Framework-Doped with Cobalt for Iodide Sensing, Langmuir. 33 (2017) 4647-4653.

[386] A. Shirmardi, M. Shamsipur, M. Akhond, J. Monjezi, Electronic tongue for simultaneous determination of cyanide, thiocyanate and iodide, Meas. J. Int. Meas. Confed. 88 (2016) 27-33.

[387] T.W. Chen, T.H. Tsai, S.M. Chen, K.C. Lin, Using PEDOT film modified electrode to monitor iodide and its enhancement of arsenite sensing, Int. J. Electrochem. Sci. 6 (2011) 2043-2057.

[388] L. Tian, L. Liu, L. Chen, N. Lu, H. Xu, Electrochemical determination of iodide on a vanadium oxide-polypropylene carbonate coated glassy carbon electrode, Talanta. 66 (2005) 130-135.

[389] I.S. El-Hallag, Electrochemical oxidation of iodide at a glassy carbon electrode in methylene chloride at various temperatures, J. Chil. Chem. Soc. 55 (2010) 67-73.

[390] F.M. Abdel-Haleem, O.R. Shehab, Comparative Study of Carbon Paste, Screen Printed, and PVC Potentiometric Sensors Based on Copper-sulphamethazine Schiff Base 
Complex for Determination of Iodide - Experimental and Theoretical Approaches, Electroanalysis. 28 (2016) 800-807.

[391] E. Cho, A. Perebikovsky, O. Benice, S. Holmberg, M. Madou, M. Ghazinejad, Rapid iodine sensing on mechanically treated carbon nanofibers, Sensors (Switzerland). 18 (2018) 1-9.

[392] B. Dielacher, R.F. Tiefenauer, J. Junesch, J. Vörös, Iodide sensing via electrochemical etching of ultrathin gold films, Nanotechnology. 26 (2015).

[393] Z.H. Ibupoto, K. Khun, M. Willander, A selective iodide ion sensor electrode based on functionalized ZnO nanotubes, Sensors (Switzerland). 13 (2013) 1984-1997.

[394] D.S. Silvester, E.I. Rogers, L.E. Barrosse-Antle, T.L. Broder, R.G. Compton, The electrochemistry of simple inorganic molecules in room temperature ionic liquids, J. Braz. Chem. Soc. 19 (2008) 611-620.

[395] F. Jalali, S. Riahi, D. Nematollahi, A Kinetic Study on Electrooxidation of PropylThiouracil: An Anti-Hyperthyroid Drug by Potassium Iodide, J. Electrochem. Soc. 160 (2013) H710-H714.

[396] S. Mukherjee, B. Ramalingam, L. Griggs, S. Hamm, G.A. Baker, P. Fraundorf, S. Sengupta, S. Gangopadhyay, Ultrafine sputter-deposited Pt nanoparticles for triiodide reduction in dye-sensitized solar cells: Impact of nanoparticle size, crystallinity and surface coverage on catalytic activity, Nanotechnology. 23 (2012).

[397] C.L. Bentley, A.M. Bond, A.F. Hollenkamp, P.J. Mahon, J. Zhang, Voltammetric Determination of the Iodide/Iodine Formal Potential and Triiodide Stability Constant in Conventional and Ionic Liquid Media, J. Phys. Chem. C. 119 (2015) 22392-22403.

[398] J. Rendl, M. Luster, C. Reiners, Serum inorganic iodide determined by paired-ion reversed-phase HPLC with electrochemical detection, J. Liq. Chromatogr. Relat. Technol. 20 (1997) 1445-1459. 
[399] T.R.I. Cataldi, A. Rubino, M.C. Laviola, R. Ciriello, Comparison of silver, gold and modified platinum electrodes for the electrochemical detection of iodide in urine samples following ion chromatography, J. Chromatogr. B Anal. Technol. Biomed. Life Sci. 827 (2005) 224-231.

[400] C. Bruggink, W.J.M. van Rossum, E. Spijkerman, E.S.E. van Beelen, Iodide analysis by anion-exchange chromatography and pulsed amperometric detection in surface water and adsorbable organic iodide, J. Chromatogr. A. 1144 (2007) 170-174.

[401] H.S. Toh, K. Tschulik, C. Batchelor-McAuley, R.G. Compton, Electrochemical quantification of iodide ions in synthetic urine using silver nanoparticles: A proof-ofconcept, Analyst. 139 (2014) 3986-3990.

[402] M. Cuartero, G.A. Crespo, E. Bakker, Paper-based thin-layer coulometric sensor for halide determination, Anal. Chem. 87 (2015) 1981-1990.

[403] E. Espada-Bellido, Z. Bi, P. Salaün, C.M.G. van den Berg, Determination of iodide and total iodine in estuarine waters by cathodic stripping voltammetry using a vibrating silver amalgam microwire electrode, Talanta. 174 (2017) 165-170.

[404] M. Khodari, U. Bilitewski, A.A.H. Basry, Screen-Printed Electrodes for Amperometric Determination of Iodide, Electroanalysis. 27 (2015) 281-284.

[405] A. V. Rzhevskaia, N. V. Shvedene, I. V. Pletnev, Anion-selective electrodes based on solidified 1,3-dihexadecylimidazolium ionic liquids with halide and pseudohalide anions, J. Electroanal. Chem. 783 (2016) 274-279.

[406] N. V. Shvedene, O.A. Avramenko, V.E. Baulin, L. G. Tomilova, I. V. Pletnev, Iodide-selective screen-printed electrodes based on low-melting ionic solids and metallated phthalocyanine, Electroanalysis. 23 (2011) 1067-1072. 
[407] H. Yajima, M. Morita, M. Hashimoto, H. Sashiwa, T. Kikuchi, T. Ishii, Complex formation of chitosan with iodine and its structure and spectroscopic properties - Molecular assembly and thermal hysteresis behavior, Int. J. Thermophys. 22 (2001) 1265-1283. 



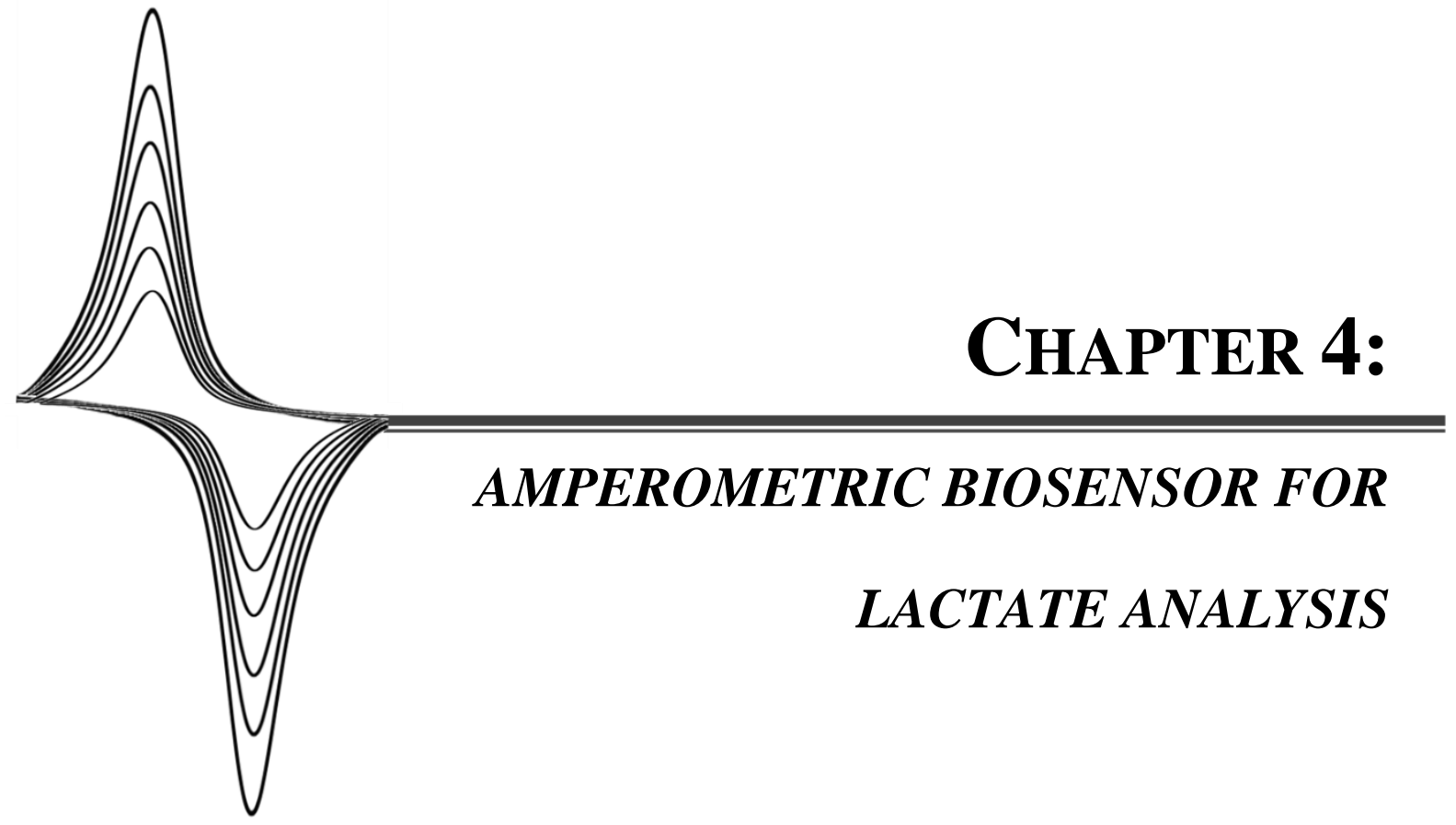



As it has been previously mentioned, lactate is an indicator of physical performance in sports activities, a disease indicator in health care applications; and an important analyte in food and cosmetic industries. Depending on the type of samples, this organic acid is routinely determined in different concentration ranges of interest.

This chapter describes the development of a SPE-based lactate biosensor to determine lactate in a broad concentration range. The $\mathrm{Cu}-\mathrm{MOF}$ (copper metallic framework) crosslinking of $0.25 \mathrm{U}$ of lactate oxidase $\left(\mathrm{LO}_{\mathrm{x}}\right)$ onto a chitosan layer, allowed to determine the enzymatic product generated on a platinum modified WE, by applying a fixed potential of $+0.15 \mathrm{~V}$ (vs SPE $\mathrm{Ag} / \mathrm{AgCl})$.

The biosensor responds linearly in two different concentration ranges: a first catalysis range, from $0.75 \mu \mathrm{M}$ to $1 \mathrm{mM}$, followed by a saturation zone from 1 to $4 \mathrm{mM}$, after which a substrate enzymatic inhibition is observed up to $50 \mathrm{mM}$. The method reproducibility was kept below $7 \%$ and a limit of detection of $0.75 \mu \mathrm{M}$ was obtained. The two ranges of analysis would allow to use the biosensor in different types of samples, with low and high content of lactate. The $\mathrm{LO}_{\mathrm{x}}$-based biosensor, successfully determined lactate in sweat and saliva, as a low-cost and noninvasive tool; also obtaining good results in wines analisis.

This work has been presented as a panel communication at XXXVI Meeting of Electrochemistry of the Spanish Royal Society of Chemistry and XVII Iberian meeting of Electrochemistry, in Vigo, Spain (July 2015) and at XXXVII Meeting of Electrochemistry of the Spanish Royal Society of Chemistry, in Alicante, Spain (July 2016). Also, it was presented as oral communication at "Convocatoria Prueba Concepto 2015", in Burgos, Spain (March 2016); at II International Congress in Health Sciences Research Towards Innovation and Entrepreneurship: Trends in Biotechnology for Medical Applications, in Covilhã, Portugal (May 2017) and at XXXVIII Meeting of Electrochemistry of the Spanish Royal Society of Chemistry and XIX Iberian meeting of Electrochemistry, Vitoria-Gasteiz, Spain (July 2017). 
Additionally, this work has been patented as "Dispositivo electródico y procedimiento de medida para la detección de ácido láctico" (PCT/ES2017/070694), and published in Talanta 188, 2018, p. 779-787. 


\subsection{INTRODUCTION}

Lactate is known as a critical metabolite biomarker of tissue oxygenation, and is studied when assessing physical performance in sports activities, to prescribe appropriate exercise intensities [1,2]. It is also analyzed in health care applications to evaluate an underlying pathology during a routine stress test $[3,4]$.

Due to a physiological accumulation, lactate relies in a process known as lactate acidosis, which takes place when the organism requires energy. This process can outcome in muscle fatigue, especially during endurance-based activities [5,6], result of $\mathrm{pH}$ decrease due to the levels of lactate in the blood and muscles [7]. Therefore, in clinical exercise and performance testing, blood lactate is the most frequently measured parameter, once it is considered as the key metabolite to finding out the degree of exhaustion of the athletes [2]. In view of the wide demand for the development of noninvasive, fast and reliable methods for monitoring lactate [8], saliva and sweat are suitable matrices for its determination, especially in sports medicine, since blood sampling can be avoided.

During exercise, lactate assessment through saliva samples has proven to be very attractive due to the high correlation with the blood levels $[9,10]$, and also given the continuous and convenient availability of this metabolite, that can vary from 0.1 to $2.5 \mathrm{mM}$ [11]. In turn, blood and sweat lactate levels have an unclear relation, although the sweat lactate is increased with exercise intensity [12-15]. Lactate constitutes about $0.28 \%$ of the organic acids content in sweat and is described to be present in concentration range from $3.7 \mathrm{mM}$ up to $50 \mathrm{mM}[16,17]$. However, this dynamic range usually reaches a maximum of $25 \mathrm{mM}$ [18].

The importance of lactate is not limited to the medical sector. This acid is found in many foods and beverages, in which concentration level indicates the freshness, stability, and quality of several products, being also added as acidulant, and flavoring agent (E270) [19]. In the winemaking industry, the course of malolactic fermentation is monitored by 
following the falling of L-malic acid and the rising of L-lactic acid. Therefore, this continuous monitoring is an important control for wine commercialization, preventing significant economic losses [20,21].

Among the various conventional analytical tools, colorimetric and chromatographic analysis of lactate are the standard methods. However, given the high complexity of these, simplest alternative such as, the use of screen-printed amperometric biosensors (SPE), have been developed for monitoring lactate [19,22-25].

The screen-printing technology offers high sensitivity and allows the easy electrode modification with nanomaterials and biocomponents, in order to attain reliable and selective devices of analysis [26]. Platinum has an important role in electrode modification, especially for oxidase enzyme based-biosensors due to their catalytic effect on their product, allowing higher sensitivities for the electrochemical measurement [27-34].

Chitosan (CS) is a biocompatible cationic polymer, successfully applied in the biomedical domain [35-37]. Heightened interest in biosensing has prompted the extensive application of CS as a matrix for enzyme immobilization in SPE [38-43], providing an excellent microenvironment for biomolecules, emerging in highly sensitive and stable devices. Moreover, it possesses an excellent membrane-forming ability, high permeability to water, good adhesion, high mechanical strength and susceptibility to chemical modifications. Cruz et al., use the permselectivity properties of this polymer to develop a fast, selective, and reversible electrochemical sensor [44].

Furthermore, a new class of porous materials called Metal-Organic Frameworks (MOF) possess several advantageous properties for sensing applications. These materials are recognized as a low-density network formed by a metallic center and an organic ligand. MOFs hold interesting properties such as ultrahigh and permanent porosity, high thermal and chemical stability, tunable pore structure and dimension, and adjustable internal surface characteristics. They have been applied to numerous areas of study, like catalysis, separation, biomedical imaging, clean energy, drug delivery and gas storage and separation $[45,46]$. In biosensing applications, these materials play a role as biomimetic components 
[47], offering improved sensitivities for detection of certain analytes [48]. Gamero et al. reported an electrochemical biosensor based on three-dimensional ordered macroporous gold film electrodes, using covalently immobilized lactate oxidase, to obtain an increased conductivity and stability for lactate sensing [49].

In the last decade, numerous reports for lactate biosensing in several matrices have been published. As an alternative for a noninvasive analysis, sweat [33,34,50-53] and saliva [54-57], are target matrices for several biosensors. Also, numerous biosensors were also released for wine and other fermented beverages [20,58,59]. However, the applicability of these devices seems to be limited to one type of matrix sample, requiring a considerable number of enzymatic units, and/or a high potential for amperometric measurement to follow the enzymatic response. Also, the range of analysis seems to be limited to values under 28 - $30 \mathrm{mM}$ of lactate $[50,60]$.

In the present work, a novel approach was exploited for lactate analysis. Based on the enzymatic kinetic of immobilized lactate oxidase $\left(\mathrm{LO}_{\mathrm{x}}\right)$, the biosensor responds linearly to lactate concentrations in two different ranges. Additional to the normal catalytic process, an inhibition process at high substrate concentrations was observed, drawing a second linear zone, able to be used directly in the analysis of samples with high lactate content. As far as we know, it is the first time that a single $\mathrm{LO}_{\mathrm{x}}$ based biosensor has demonstrated its applicability in the determination of lactate in diversified samples, using a low number of enzymatic units, with two distinct analysis ranges, allowing in this case, the quantification of lactate in wines, saliva and sweat samples. The design, characterization and evaluation of the dual range $\mathrm{LO}_{\mathrm{x}}$ biosensor are described in the following sections. 


\subsection{EXPERIMENTAL SECTION}

\subsubsection{Chemicals and reagents}

Lactate oxidase $\left(\mathrm{LO}_{\mathrm{x}}\right)$ from pediococcus sp. (E.C. 1.1.3.2.) lyophilized powder, 28 units/mg solid, chitosan, glutaraldehyde (GA), bovine serum albumin (BSA), terephthalic acid (1,4 - $\mathrm{H}_{2} \mathrm{BDC}$ ), triethylenediamine (TED), N,N-Dimethylformamide (DMF), L-(-)-malic acid, maleic acid, gallic acid, sodium pyruvate, resveratrol and $\mathrm{D}(+)$-Glucose monohydrate, were purchased from Sigma-Aldrich (Steinheim, Germany). Potassium hexachloroplatinate (IV), L-histidine monohydrochloride monohydrate, sulfuric acid, urea, ascorbic acid, sodium phosphate dibasic dihydrate, sodium phosphate monobasic dihydrate, copper nitrate trihydrate, ethanol and potassium chloride were obtained from Merck (Darmstadt, Germany). From Panreac-Applichem (Darmstadt, Germany) were purchased L-lactic acid (85\%), succinic acid, citric acid anhydrous, ortho-phosphoric acid (85\%), sodium chloride and sodium hydroxide. Creatinine was obtained from (Barcelona, Cromatest), and Uric Acid from Alfa Aesar. Quercetin dihydrate from Extrasynthese (Genay, France). Acetic acid from VWR (France).

In the fabrication of screen-printed electrodes inks used, namely Electrodag PF-407 A (carbon ink), Electrodag 6037 SS (silver/silver chloride ink), Electrodag 418 (silver ink) and Electrodag 452 SS (dielectric ink), were supplied by Achenson Colloiden (Scheemda, Netherlands).

The cooper metal framework (Cu-MOF) was synthesized according to the previously reported method by [61] and [48]. Briefly, copper nitrate trihydrate $(0.493 \mathrm{~g}), 1.4-\mathrm{H}_{2} \mathrm{BDC}$ $(0.453 \mathrm{~g})$ and TED $(0.32 \mathrm{~g})$ were dissolved in $100 \mathrm{~mL}$ of DMF, and the mixture was sonicated to obtain a homogenous solution. Then the solution was heated at $120^{\circ} \mathrm{C}$ for 36 h. After cooling at room temperature, the obtained blue crystalline power was collected, washed with DMF and water several times and dried under vacuum overnight.

All the reagents used were of analytical grade and Milli-Q water (Millipore, Bedford, USA) was employed for preparing all solutions. 
Human saliva and sweat samples were collected from healthy volunteers and were stored at $-20^{\circ} \mathrm{C}$. The sweat samples were collected in vials, from volunteers' front head, immediately after running. Also, saliva samples were collected directly in vials and stored until used. The wine samples were obtained from the local market and stored at $4^{\circ} \mathrm{C}$.

\subsubsection{Apparatus}

Voltammetric measurements were performed using an Autolab PGSTAT128N electrochemical system with the General Purpose Electrochemical System (GPES) software version 4.9 (Echo Chemie, Utrecht, The Netherlands). A PalmSens ${ }^{\circledR}$ portable electrochemical potentiostat with the PS Trace program (PalmSens ${ }^{\circledR}$ Instruments BV, Houten, The Netherlands) was used for chronoamperometric measurements.

Reversed phase HPLC analysis was performed with a liquid chromatograph Flexar PerkinElmer ${ }^{\circledR}$ (Connecticut, U.S.A), consisting of a binary pump, manual sampler, and UV detector. Chromatographic separation was carried out at an ACE 5 C18 Column $\left(\mathrm{ACE}^{\circledR}\right.$ HPLC Columns, Aberdeen, Scotland), $250 \mathrm{~mm} \times 4.6 \mathrm{~mm}$ and with particle size of $5 \mu \mathrm{m}$.

Microscopy imaging and elemental composition analysis were performed using a scanning electron microscope (SEM) JEOL JSM-6460LV with an INCA elementary X-ray analysis system Oxford Instruments (Abingdon-on-Thames, UK). The X-ray analysis was performed at three different zones of each working electrode with a zoom of x500.

\subsubsection{SPE manufacturing}

Screen-printed electrodic systems, SPE, were produced following a previously described procedure [62]. Sequential deposition of the inks, which defines conductive silver tracks, $\mathrm{Ag} / \mathrm{AgCl}$ reference electrode, carbon counter and working electrodes and dielectric layer, was performed on polyester films (HIFI Industrial Film, Dardily France). 


\subsubsection{Electrode Modification}

\subsubsection{Platinum coating (Pt/SPCE)}

To achieve a homogenous Pt coating onto the carbon surface of the working electrode, a volume of $200 \mu \mathrm{L}$ of a $1 \mathrm{mM}$ platinum solution in $0.1 \mathrm{M} \mathrm{KCl}$ was deposited onto the electrodic system and cyclic voltammetric measurements were performed from 0.5 to -0.7 $\mathrm{V}$ (vs SPE $\mathrm{Ag} / \mathrm{AgCl}$ ), with a scan rate of $0.002 \mathrm{~V} \mathrm{~s}^{-1}$. The experiments were conducted for $5,10,15,20,25,30,45,55,80$ and 100 scans. The procedure, a modification of the one described for Compton [63], proves to be more efficient for the developed system (section 4.3.1.).

\subsubsection{Preparation of enzymatic electrodes $\left(L O_{x}-\mathrm{Cu}-\mathrm{MOF} / \mathrm{CS} / \mathrm{Pt} / \mathrm{SPCE}\right)$}

After the platinum modification, the Pt/SPCE electrode was rinsed with Milli-Q water. Then the electrode was dried and $2 \mu \mathrm{L}$ of chitosan $(0.6 \%$ (w/v) in $0.5 \%$ acetic acid) were cast onto the electrode surface and dried at room temperature. Following, the enzyme was crosslinked on the CS/Pt/SPCE surface, using an immobilization solution, containing $3 \mu \mathrm{L}$

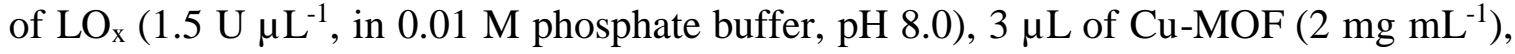
$0.56 \mu \mathrm{L}$ of BSA ( $3 \%(\mathrm{w} / \mathrm{v}))$ and $0.56 \mu \mathrm{L}$ of GA $(2.5 \%(\mathrm{v} / \mathrm{v})) .1 .20 \mu \mathrm{L}$ of this solution, was cast onto the electrode surface, and the modified electrode, $\mathrm{LO}_{\mathrm{x}}-\mathrm{Cu}-\mathrm{MOF} / \mathrm{CS} / \mathrm{Pt} / \mathrm{SPCE}$, was dried and stored at $4{ }^{\circ} \mathrm{C}$ in a refrigerator until use.

\subsubsection{Electrochemical measurements}

Amperometric measurements were carried out in a bath system. All measurements were made at room temperature in a cell containing $5 \mathrm{~mL}$ of supporting electrolyte (phosphate buffer at $0.1 \mathrm{M}, \mathrm{pH} 7.0$ ), with constant stirring. The working electrode operated at +0.60 and $+0.15 \mathrm{~V}$ (vs SPE Ag/AgCl), in the case of $\mathrm{LO}_{\mathrm{x}} / \mathrm{SPCE}$ and $\mathrm{LO}_{\mathrm{x}} / \mathrm{Pt} / \mathrm{SPCE}$, respectively. The corresponding sample was added after reaching a stable baseline. 
Wine and sweat samples were spiked and analyzed without any pretreatment. Only the saliva sample was spiked after centrifuging, to avoid high viscosity. Biosensing analysis of the real samples was carried out using the standard addition method.

Voltammetric measurements were recorded, between - $0.2 \mathrm{~V}$ to $+0.9 \mathrm{~V}$ (vs SPE $\mathrm{Ag} / \mathrm{AgCl}$ ) at different scan rates from $0.02 \mathrm{~V} \mathrm{~s}^{-1}$ to $0.15 \mathrm{~V} \mathrm{~s}^{-1}$ in a solution containing $2 \mathrm{mM}$ of lactate in supporting electrolyte.

\subsubsection{HPLC measurements}

Before use, the column was cleaned with water and methanol/water (85/15), during 30 minutes for each step. Then, isocratic elution was performed using a phosphate buffer $(0.2 \%(\mathrm{v} / \mathrm{v})$ of phosphoric acid and $0.16 \%(\mathrm{w} / \mathrm{v})$ of dihydrogen phosphate in water, $\mathrm{pH} 2.0)$, at $1.2 \mathrm{~mL} \mathrm{~min}^{-1}$. Injection volume was $20 \mu \mathrm{L}$, and the quantification wavelength was set at $216 \mathrm{~nm}$. The amount of lactate was quantified using Chromera chromatography software, determining the lactate/maleic acid peak area ratio, where maleic acid is the internal standard. The concentration of lactate in the samples was determined from the calibration curve of the peaks at the retention time of 4.6 and 6.7 minutes, for lactate and maleic acid, respectively (Figure 4.1) [64].

Prior to HPLC analysis, all samples were centrifuged at $13.2 \mathrm{rpm}$ at $4{ }^{\circ} \mathrm{C}$, during $30 \mathrm{~min}$., and the supernatant filtered through sterile cellulose acetate $0.45 \mu \mathrm{m}$-membrane syringe filter. The samples were diluted; 7.5 times for red wine and sweat samples, and 2 times for white wine and saliva; and finally enriched with $10 \mathrm{ppm}$ of maleic acid. All samples, standards and chromatographic phases were previously sonicated. 


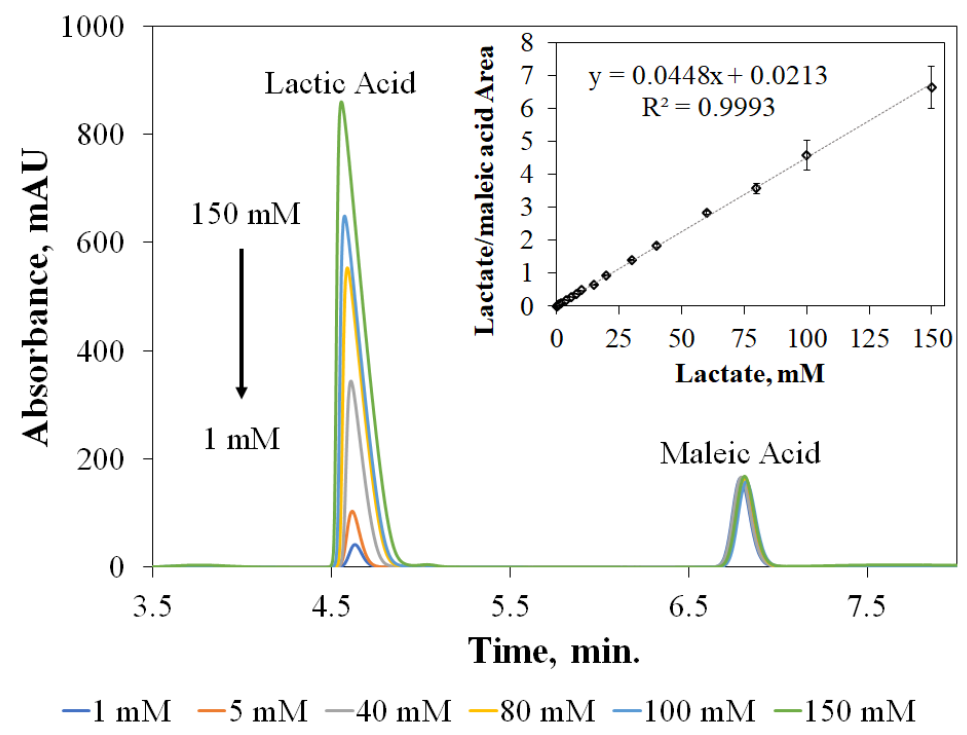

Figure 4.1. Representative chromatogram from HPLC analysis for lactate calibration. Peak at 4.6 minutes corresponding to lactate 1, 5, 40, 80, 100 and 150 mM; peak at 6.7 minutes corresponding to internal standard of maleic acid. Inset: Calibration curve of lactate, from 0.05 to $150 \mathrm{mM}$, based on the lactate/maleic acid peak area for each lactate concentration. Error bars corresponding to standard deviation for each concentration.

\subsection{RESULTS AND DISCUSSION}

Lactate Oxidase $\left(\mathrm{LO}_{\mathrm{x}}\right)$, is a flavoprotein recognized for its ability to catalyze the oxidation of lactate to pyruvate in the presence of oxygen, as shown in the previous equation 3.12 (page 59). In the proposed method, a biosensor based on the immobilization of $\mathrm{LO}_{\mathrm{x}}$ on a platinum surface was used as a sensing interface to catalyze the electrochemical oxidation of hydrogen peroxide (Equation 3.13, page 59). The biosensor allows the selective determination of lactate by monitoring the amperometric oxidation of the hydrogen peroxide, produced from the enzymatic reaction.

It was found that, depending on lactate concentration, $0.25 \mathrm{U}$ of immobilized $\mathrm{LO}_{\mathrm{x}}$ leads to different responses. In the first stage of enzymatic catalysis, a linear increment in the current 
signal was observed, for lactate concentrations from $0.75 \mu \mathrm{M}$ to $1 \mathrm{mM}$. After a saturation zone, between 1 and $4 \mathrm{mM}$, a linear decrease in current intensity values takes place, for lactate ranging between 4 and $50 \mathrm{mM}$, as a consequence of a substrate inhibition process.

Copper metallic framework (Cu-MOF) and Chitosan (CS) were used to improve the biosensor, proving to considerably increase device performance. Therefore, a deep study of each component influence was carried out, as described in the next sections.

\subsubsection{Electroactive surface area determination}

Platinum surfaces are known to notably favor the oxidation of hydrogen peroxide since the potential required for oxidation corresponds to the platinum oxide (PtO) film formation [32]. The modification of the carbon working surface with a Pt coating improves the electrochemical signal for lactate determination. Therefore, in order to survey Pt influence in the electrochemical response, the electroactive surface area (ESA) was determined for the different number of voltammetric cycles used in Pt coating of working electrodes [65]. Several theories have been proposed to determine the electroactive surface area (ESA) of electrodes. ESA measurements can be based on chemisorption phenomena of a chemical bond between the catalyst surface and the adsorbate. As Pt provides a well-developed region for oxide monolayer reduction, the method applied evaluates the peak charge of $\mathrm{PtO}$ at the Pt/SPCE $[65,66]$.

To determine the ESA of Pt/SPCE, cyclic voltammetric measurements were conducted in an acidic media $\left(0.1 \mathrm{M} \mathrm{H}_{2} \mathrm{SO}_{4}\right)$ from -0.3 to $1.3 \mathrm{~V}$ and the reduction peaks for platinum oxide (at approximately $0 \mathrm{~V}$ ), were registered.

It was possible to calculate the ESA surface area by applying the equation 4.1 [66]:

$$
E S A\left(c m^{2}\right)=\frac{Q_{o}(\mu C)}{420\left(\mu C \mathrm{~cm}^{-2}\right)}
$$


where, $Q_{o}$ is the charge associated with surface oxidation, and $420 \mu \mathrm{C} \mathrm{cm}^{-2}$ is the charge associated with the full monolayer of oxygen corresponding to $\mathrm{PtO}$, which requires two electrons per site for its removal.

By increasing the number of voltammetric cycles the ESA value rises, given the amount of platinum deposited onto the carbon surface. The ESA value obtained by electrode modification with $\mathrm{Pt}$ is noticeably increased in comparison with the geometric area $(0.126$ $\mathrm{cm}^{2}$ ) (Figure 4.2). It has been previously found that the electroactive area can be increased about 1000 times comparatively to the geometric one [67]. Area evolution remained steady after 55 scans, and ESA value was estimated to increase hundredfold, compared to the geometric one. This plateau is defined by the electrode surface saturation for Pt deposition.

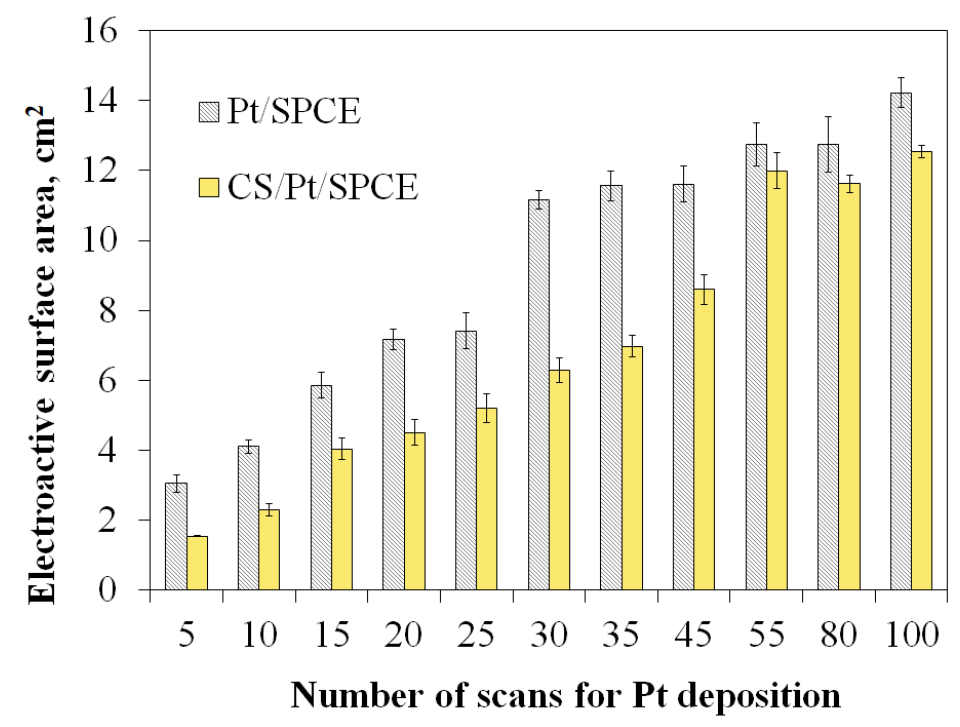

Figure 4.2. Evolution of ESA with the number of $C V$ scans for platinum coating deposition, in the presence (CS/Pt/SPCE) and absence of chitosan (Pt/SPCE). Each point corresponds to the average value of 10 measurements of $Q_{0}$, carried out in three different electrodes $(n=3, R S D<10 \%)$.

Besides the contribution in terms of electroactive area, which allows for a more effective determination of the enzymatic generated $\mathrm{H}_{2} \mathrm{O}_{2}$, this metallic coating also greatly 
contributes to an improvement of the optimal work potential since it decreases from +0.60 to $+0.15 \mathrm{~V}$. This new potential value introduces additional advantages in terms of electrochemical response specificity, also offering satisfactory current outputs.

Calibration curves were obtained to evaluate the effect of the number of scans in the Pt deposition process on the electrochemical behavior of the lactate biosensor. The higher the number of scans, the higher the current values that were found, increasing sensitivity for the first measurement range of biosensor (from $0.75 \mu \mathrm{M}$ to $1 \mathrm{mM}$ ). However, for lactate concentrations from 4 to $50 \mathrm{mM}$, it was observed that the inhibitory response of the biosensor was weakly altered by the amount of deposited $\mathrm{Pt}$, indicating that the process is controlled by the decrease in enzymatic activity.

The Pt coating process is time consuming and does not greatly affect the inhibition process of $\mathrm{LO}_{\mathrm{x}}$. In light of this, 10 voltammetric cycles for SPCE modification with Pt were chosen, since it is less time consuming for electrode modification and the biosensor responds adequately to both low and high lactate concentrations, by increasing the ESA about 33 times relative to the geometric area. In agreement with electrochemical data, SEM and Xray analysis shows an efficient nucleation of Pt onto working SPCE, exhibiting a satisfactory content of Pt nanostructures by using 10 voltammetric cycles of deposition, which favors the enzymatic product oxidation (Figures 4.3 and 4.4).
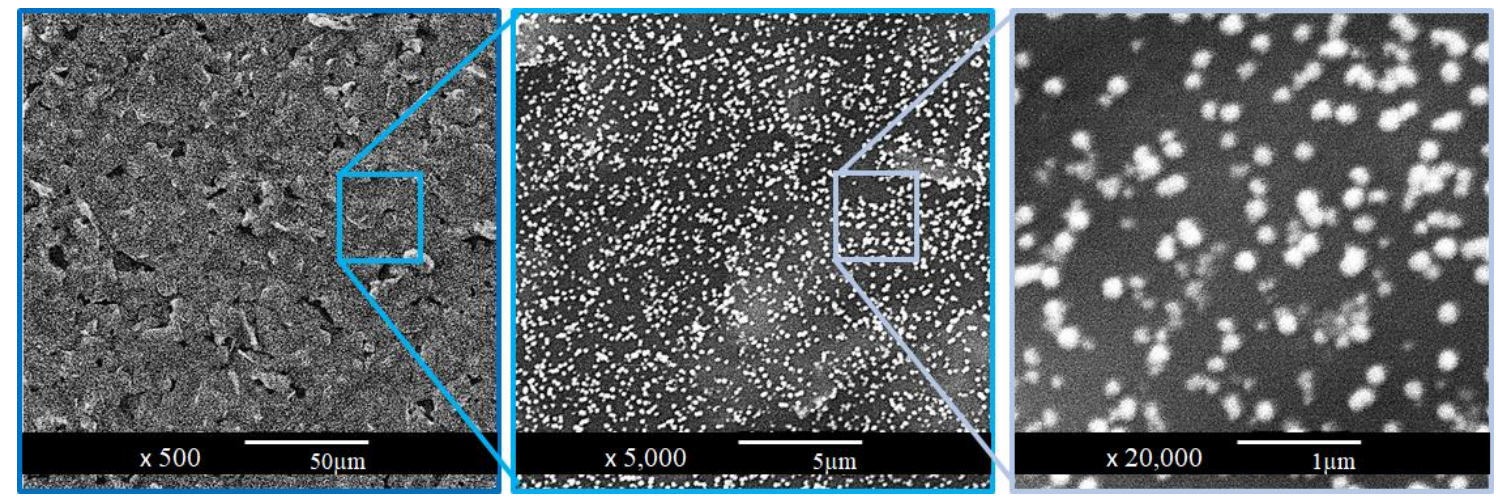

Figure 4.3. SEM micrographs of the working Pt/SPCE, modified with 10 cycles of deposition. Pt nanostructures at three different magnifications. 


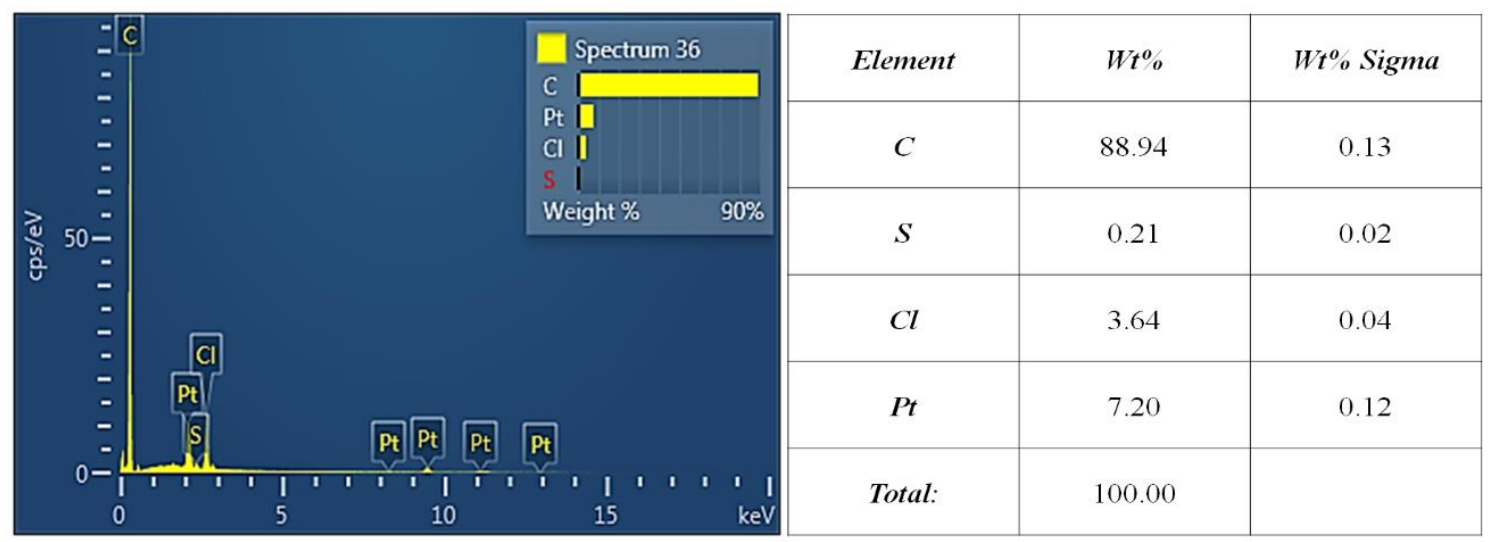

Figure 4.4. X-Ray elemental analysis of Pt/SPCE surface, modified with 10 cycles for Pt deposition. Elemental composition of Pt/SPCE working electrode surface.

\subsubsection{Chitosan influence in amperometric measurements}

The presence of CS is proven to be essential for an adequate amperometric response, by affecting the enzymatic behavior in several aspects. It was observed that this membrane acts like a barrier, delaying the displacement rate of the enzymatic reaction product, $\mathrm{H}_{2} \mathrm{O}_{2}$, to the $\mathrm{Pt}$ surface and the amperogram resolution increased in the range of lactate concentrations corresponding to the enzyme inhibition (4 to $50 \mathrm{mM}$ ). When tested to evaluate enzymatic activity, the CS membrane marks a delay in electrochemical signal acquisition, proving that the polymeric film hinders the arrival of the analytes to the electrode surface. In fact, the linear relation between the cyclic voltammetric peak current and the square root of the scan rate according to Randles-Sevcik equation [68], demonstrates that a diffusion-controlled reaction is taking place on the Pt electrode surface.

The study of electroactive area was conducted in both the presence and absence of chitosan, since this membrane can lead to a lower Pt ESA available for the electrochemical oxidation of $\mathrm{H}_{2} \mathrm{O}_{2}$. In fact, the presence of a CS layer results in slightly lower ESA values, probably due to a partial surface covering with the polymeric molecules. Nevertheless, the same trend of the electroactive area evolution was attained for CS/Pt/SPCE (Figure 4.2). 
On the other hand, the enzymatic activity of $\mathrm{LO}_{\mathrm{x}}$ is positively influenced by this compound, probably due to the biocompatibility of this natural polymer. The use of CS combined with a Pt coating on the electrode surface makes the developed biosensor more sensitive for both concentration ranges, $0.75 \mu \mathrm{M}$ to $1 \mathrm{mM}$, and from 4 to $50 \mathrm{mM}$. Therefore, several CS concentrations of $0,0.2,0.6,1$ and $2 \%(\mathrm{w} / \mathrm{v})$ in $0.5 \%$ acetic acid, were tested on the $\mathrm{Pt}$ surface, before the enzymatic immobilization (Figure 4.5).

A considerable increase in generated current was observed in the presence of CS. Improved values for lactate biosensing in both analysis ranges were found for 0.6 and $1 \%$ of CS. Nevertheless, a slow process for $1 \%$ CS was observed, affecting the experimental time. Also, higher amount of CS seems to be connected to higher noise in the amperometric measurement. Bearing this in mind, $0.6 \% \mathrm{CS}$ was selected as the optimal membrane, given that it has a higher sensitivity for lactate with a good amperometric resolution (Figure 4.7. A, orange line).
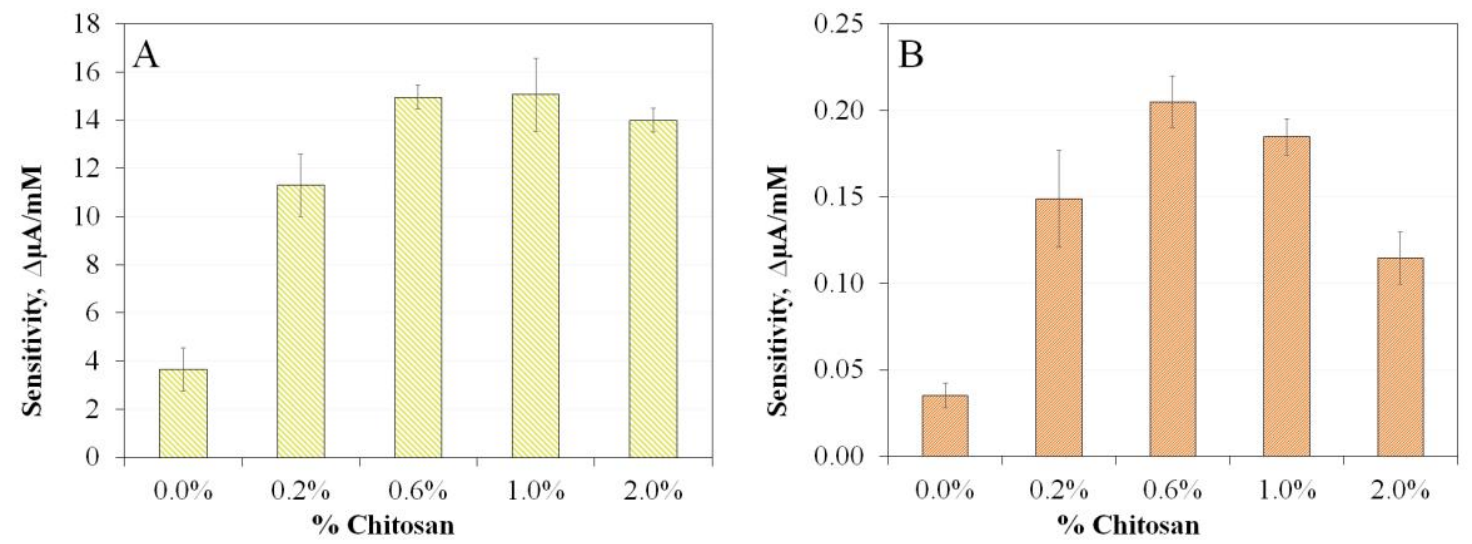

Figure 4.5. Optimization of chitosan layer deposited in the working. Influence on the sensitivity for lactate determination in catalysis (A) and inhibition (B) zones. Error bars corresponding to standard deviation for each chitosan concentration.

The role of this membrane was also studied when deposited in different steps of the immobilization procedure, and the effect in the amperometric signal registered. When 
deposited after enzyme immobilization, the registered current was lower than that observed when the biocomponent was first immobilized. This probably reflects the $\mathrm{LO}_{\mathrm{x}}$ activity loss as consequence of the $\mathrm{pH}$ of CS solution. The same behavior was found for the enzymatic sandwiching between two CS deposits resulting in a lag response for lactate sensing.

\subsubsection{Optimization of immobilization procedure}

\subsubsection{Copper metal-organic framework influence in amperometric measurement}

Additionally, in this work the use of $\mathrm{Cu}-\mathrm{MOF}$ proves to be favorable in terms of the biosensor's inhibition signal sensitivity and stability. According with the literature, this MOF present a brick-like morphology, and is able to form a film layer in presence of chitosan, also this material can influence the charge transfer process $[48,49]$. The study of the metallic framework influence was carried out using different amounts of $\mathrm{Cu}-\mathrm{MOF}$, ranging from $0,1,2,3$ and $4 \mathrm{mg} \mathrm{mL}^{-1}$, in the immobilization solution (Figure 4.6). Despite other $\mathrm{Cu}-\mathrm{MOF}$ concentrations leading to higher slope values for lactate sensing through inhibition, they also show a higher variability of the electrochemical response. The optimal balance between stability and sensitivity for lactate biosensing was found to be $2 \mathrm{mg} \mathrm{mL}^{-1}$ of $\mathrm{Cu}-\mathrm{MOF}$.

In figure 4.7. A (green line), we can observe an imperceptible influence in the catalytic zone barely affecting the sensitivity. This data contrast with a positive evolution of biosensor sensitivity for $\mathrm{LO}_{\mathrm{x}}$ inhibition response. When added to the enzymatic immobilization step, $\mathrm{Cu}-\mathrm{MOF}$ proves to result in higher sensitivity of immobilized enzyme to inhibitory concentrations of lactate, increasing the slope obtained in this second zone. Comparing with the normal glutaraldehyde crosslinking onto the working electrode, higher sensitivities and improved current decrease steps, were obtained. 

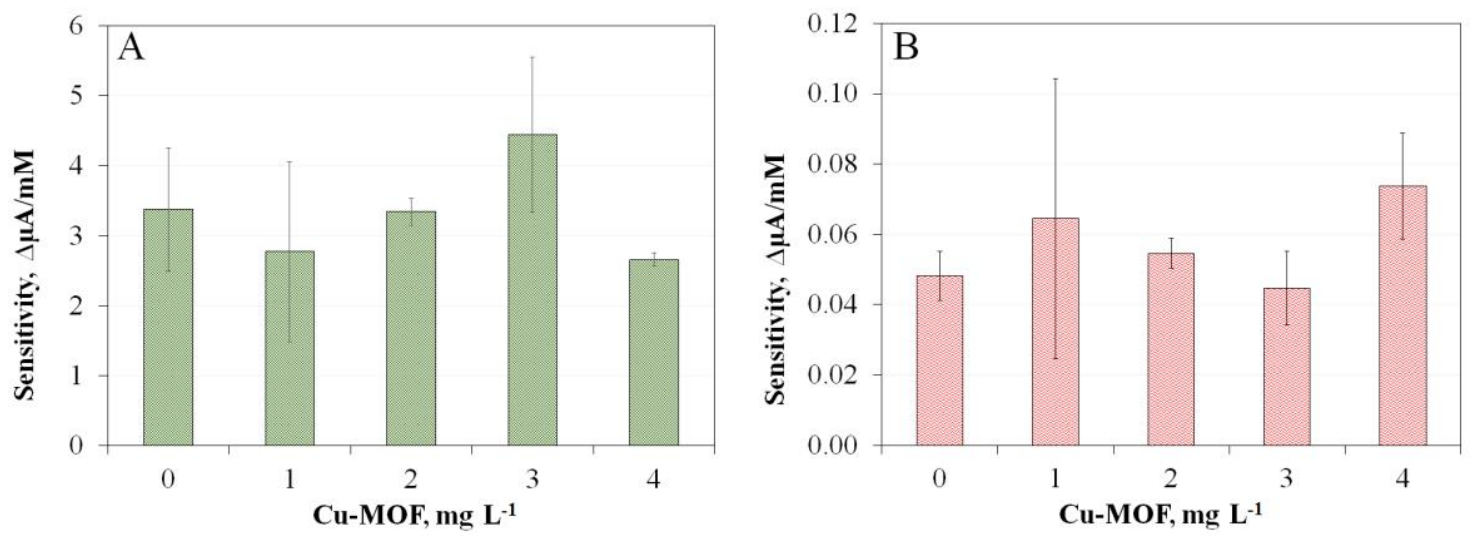

Figure 4.6. Optimization of Cu-MOF used in crosslinking mixture. Influence on the sensitivity for lactate determination in catalysis (A) and inhibition (B) zones. Error bars corresponding to standard deviation for each $\mathrm{Cu}-\mathrm{MOF}$ concentration.
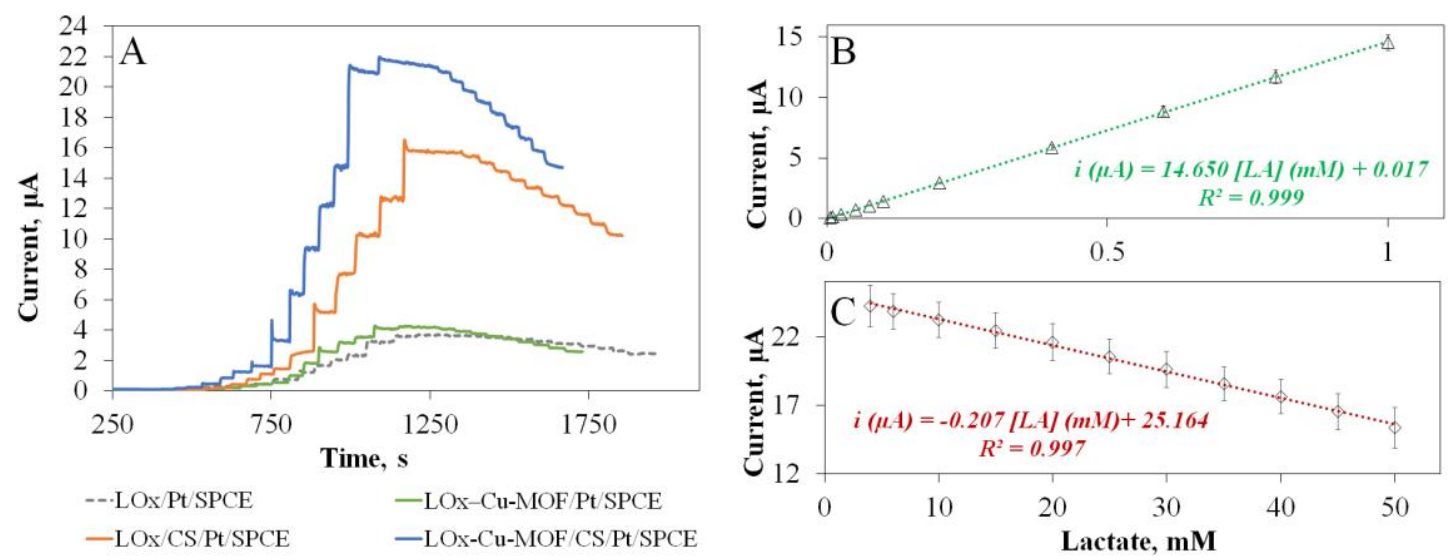

Figure 4.7. A: Amperograms obtained at different $L O_{x}$ based biosensors in $5 \mathrm{~mL}$ of phosphate buffer $\mathrm{pH}$ 7.0, for successive additions of lactate ranging from $0.75 \mu \mathrm{M}$ up to $50 \mathrm{mM}$. $E_{\text {app }}: 0.15 \mathrm{~V}$; (grey) $L O_{x} / P t / S P C E$; (green) $L O_{x}-C u-M O F / P t / S P C E$; (orange) $L O_{x} / C S / P t / S P C E$, and (blue) $L O_{x^{-}}$ Cu-MOF/CS/Pt/SPCE. Calibration curves in catalysis (B) and inhibition (C) zones obtained in optimal conditions, corresponding to amperogram (blue) $L O_{x}-C u-M O F / C S / P t / S P C E$.

Regarding the configuration of the sensing interface is noted that, by associating the individual contribution of each component with the all components used at time, the $\mathrm{LO}_{\mathrm{x}^{-}}$ 
based biosensor sensitivity is increased in both ranges for analysis (Figure $4.7 \mathrm{~A}$, blue line). It should be noted that the concentration ranges for analysis were maintained, indicating that the amount of enzyme is the responsible of this property. An enhanced signal is clearly observed when the $\mathrm{Cu}-\mathrm{MOF}$ crosslinking is performed on a CS membrane, showing the metallic framework contribution in terms of sensitivity for the catalysis zone. This may be possibly attributed to a better orientation of the enzymatic structure in presence of the $\mathrm{Cu}$ MOF by preventing the active center hinder by the CS membrane.

\subsubsection{Enzymatic crosslinking}

The design and manufacture of enzymatic biosensors is deeply influenced by the immobilization of the enzyme on the electrochemical transducer, allowing an increase in stability and selectivity [69]. In this work, $\mathrm{LO}_{\mathrm{x}}$ was immobilized onto the CS/Pt/SPCE by $\mathrm{Cu}-\mathrm{MOF}$ crosslinking using GA as a bifunctional reagent mixed with a non-active protein, BSA. Despite the previously optimized amount of each crosslinking component (data not shown), elimination of GA fully from the immobilization procedure could result in higher biosensor performance since this bifunctional reagent is known for having denaturing properties. Furthermore, the recognized porosity of the MOF structures suggests that this type of components could also behave as an immobilization frame, avoiding the need of GA as bifunctional agent. Therefore, electrochemical measurements were carried out in the presence and absence of the crosslinking agents when $\mathrm{Cu}-\mathrm{MOF}$ was present. However, the biosensor displays a non-favorable response when the crosslinking agents are absent. In fact, the biosensor's performance is altered, resulting in less sensitive in the inhibitory range. Also, an increase in measurement noise was observed, by signal to noise ratio decrease on those conditions, hindering an ideal visualization of amperometric steps.

\subsubsection{Biosensor characterization}

The accuracy of the developed method was calculated in terms of reproducibility and repeatability. Under optimum conditions, several calibration curves were obtained varying 
the concentration range from $0.75 \mu \mathrm{M}$ up to $1 \mathrm{mM}$, and from 4 to $50 \mathrm{mM}$, to study reproducibility in both catalysis and inhibitory zones respectively (Figure $4.7-\mathrm{B}$ and C). The reproducibility achieved in terms of RSD associated with the slopes of the calibration curves, recorded using different biosensors, was $5.03 \%$ in the catalysis calibration range, while for the inhibitory lactate concentrations an RSD of $6.82 \%$ was attained.

Despite the disposable nature, under optimum operating conditions, a single biosensor submitted to successive calibration measurements, in the first range, only lose more than $10 \%$ of sensitivity after the tenth measurement (Figure 4.8 ).

The linear regression parameters between lactate concentration and the analytical signal for catalysis range were used to determine the limit of detection (LOD). Using the equation $\mathrm{LOD}=3 S_{x / y} / b$, where $S_{x / y}$ is the estimated standard deviation of the minimum concentration detected and $b$ the average slope of three catalysis calibration curves, the LOD obtained was of $0.75 \mu \mathrm{M}[70,71]$.

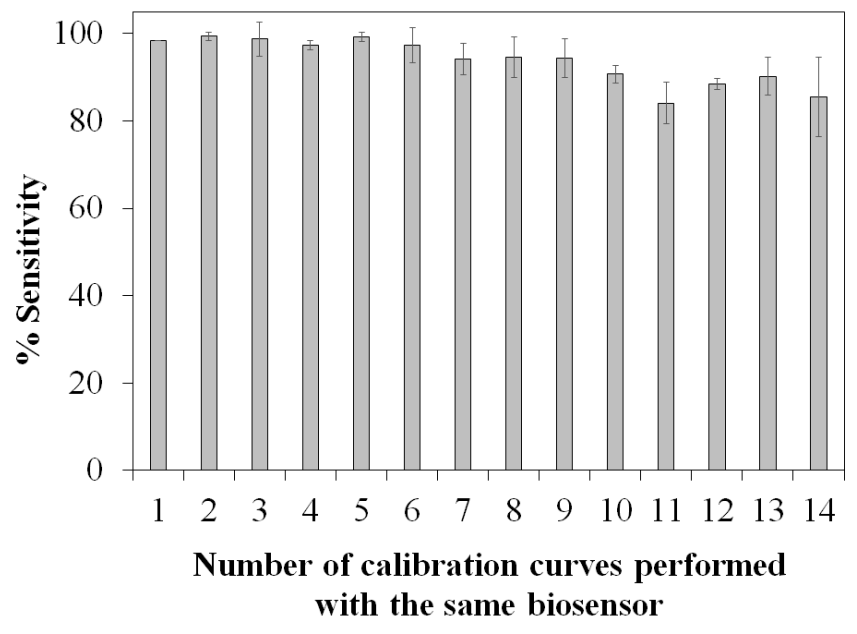

Figure 4.8. Stability of $L_{x}-C u-M O F / C S / P t / S P C E$ biosensor during repeated measurements. Results expressed as average percentage of sensitivity obtained for three distinct biosensors. Error bars corresponding to standard deviation of calibration slopes. 


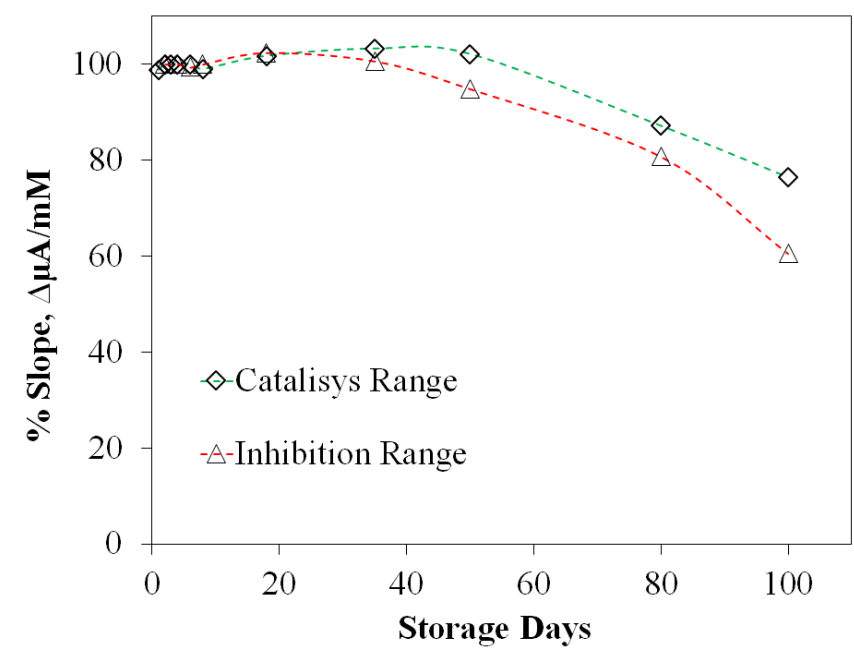

Figure 4.9. Stability of $\mathrm{LO}_{x}-\mathrm{Cu}-\mathrm{MOF} / \mathrm{CS} / \mathrm{Pt} / \mathrm{SPCE}$ biosensor. Results expressed as percentage of slope maintenance over days for both zones, when stored at $4^{\circ} \mathrm{C}$ until use.

Table 4.1. Calibration parameters of $\mathrm{LO}_{x}-\mathrm{Cu}-\mathrm{MOF} / \mathrm{CS} / \mathrm{Pt} / \mathrm{SPCE}$ biosensor for lactate determination in buffered media $(n=3)$. (*) Inhibition response at physiological level of biological interferents.

\begin{tabular}{|c|c|c|c|c|c|}
\hline $\begin{array}{c}\text { Working } \\
\text { Potential (V) }\end{array}$ & Analysis Range & $\begin{array}{l}\text { Sensitivity } \\
\left(\mu A m M^{-1}\right)\end{array}$ & $R^{2}$ & $R S D(\%)$ & $\begin{array}{l}L O D \\
(\mu M)\end{array}$ \\
\hline \multirow{3}{*}{$+0.15 \mathrm{~V}$} & Catalysis & $14.650 \pm 0.721$ & 0.999 & 5.03 & 0.75 \\
\hline & Inhibition & $0.207 \pm 0.016$ & 0.997 & 6.82 & - \\
\hline & Inhibition $^{*}$ & $0.0123 \pm 0.00051$ & 0.999 & 5.56 & - \\
\hline
\end{tabular}

In terms of lifetime, the biosensors prove to maintain approximately $100 \%$ response sensitivity, reflected in terms of calibration curve slope, for both zones up to 50 days, when stored at $4^{\circ} \mathrm{C}$ until use (Figure 4.9). The long-term stability achieved with the $\mathrm{LO}_{\mathrm{x}}-\mathrm{Cu}-$ $\mathrm{MOF} / \mathrm{CS} / \mathrm{Pt} / \mathrm{SPCE}$, improve the commercial value of the sensor and the effectiveness in lactic acid monitoring during the malolactic fermentation, once this process usually takes 
place for up to 40 days in most wines [72]. The analytical parameters that characterize the sensor performance are shown in table 4.1.

In table 4.2, the main characteristics of the developed biosensor are compared with other $\mathrm{LO}_{\mathrm{x}}$ - based electrochemical biosensors reported in the literature. The approach presented in this work outperforms other devices in terms of operational stability and in terms of biosensor performance. The proposed biosensor is the only one that offers the possibility to determine lactate in different real samples, using two different concentration ranges of interest, a catalytic one from $0.75 \mu \mathrm{M}$ to $1 \mathrm{mM}$, and an inhibitory range from 4 to $50 \mathrm{mM}$, both in the same device. Our device is the only able to determine high content of lactate, reaching an upper limit of analysis of $50 \mathrm{mM}$. In addition, the enzymatic units used of 0.25 $\mathrm{U}$, are considerably lower than other approaches, that can reach similar ranges, resulting in an economic device. In this way, is possible to attend different end-users, once the biosensor can cover a broad range of analysis, being useful in both biological and food analysis, with possibility to perform continuous measurements. 
Table 4.2. Comparison of analytic characteristics of various lactate oxidase based-amperometric biosensors.

\begin{tabular}{|c|c|c|c|c|c|c|c|c|c|}
\hline $\begin{array}{l}\text { Enzymatic } \\
\text { units used } \\
\text { per sensor }\end{array}$ & $\begin{array}{c}\text { Working } \\
\text { potential } \\
(V)\end{array}$ & Electrode modifications & $\begin{array}{l}\text { Linearity } \\
\quad(m M)\end{array}$ & $\begin{array}{l}L O D \\
(\mu M)\end{array}$ & $\begin{array}{c}\text { Storage } \\
\text { stability } \\
\text { (Days) }\end{array}$ & $\begin{array}{l}\text { Sensitivity } \\
\left(\mu A \boldsymbol{A} M^{-1}\right)\end{array}$ & $\begin{array}{c}R S D \\
(\%)\end{array}$ & Applications & References \\
\hline \multirow[b]{2}{*}{0.25} & \multirow[b]{2}{*}{0.150} & \multirow{2}{*}{$\mathrm{LO}_{x}-\mathrm{Cu}-\mathrm{MOF} / \mathrm{CS} / \mathrm{Pt} / \mathrm{SPCE}$} & $0.00075-1.00$ & 0.75 & \multirow[b]{2}{*}{50} & 14.650 & 5.03 & Sweat, Saliva, Red and & This work \\
\hline & & & $4.00-50.00$ & & & 0.207 & 6.82 & White Wines & \\
\hline 10.00 & -0.100 & $\begin{array}{c}\mathrm{PS} / \mathrm{MWCNT} / \mathrm{Fc} / \mathrm{LO}_{\mathrm{x}} / \mathrm{HRP} / \\
\mathrm{SPCE}\end{array}$ & $0.011-0.06$ & 0.56 & 15 & $\begin{array}{c}1168.8 \\
\mu \mathrm{A} \mathrm{M}^{-1} \mathrm{~mm}^{-2}\end{array}$ & 2.70 & Wine and beer & [20] \\
\hline 0.20 & 0.075 & $\mathrm{PPY}_{3 \mathrm{DOM}} / \mathrm{HRP} / \mathrm{LO}_{\mathrm{x}} / \mathrm{Au}$ & $0.01-0.10$ & 0.52 & 40 & 13.500 & 4.00 & Wines & [72] \\
\hline 2.13 & 0.730 & $\mathrm{LO}_{x} / \mathrm{DTSP} / \mathrm{Au}_{3 \mathrm{DOM}}$ & $0.00-1.30$ & 3.93 & 30 & 1.630 & 3.50 & $\begin{array}{c}\text { Lack of real samples } \\
\text { analysis }\end{array}$ & [49] \\
\hline 20.00 & -0.050 & $\mathrm{LO}_{\mathrm{x}} / \mathrm{PuBnc} / \mathrm{SPCE}$ & $0.01-0.5$ & 10.00 & 21 & $\begin{array}{c}6.380 \\
\mathrm{~mA} \mathrm{M}^{-1} \mathrm{~cm}^{-2}\end{array}$ & NR & Blood & [73] \\
\hline $60.00^{\mathrm{a}}$ & 0.650 & $\mathrm{LO}_{x} / \mathrm{PPr}-\mathrm{PPA} / \mathrm{Ptw}$ & $0.00-1.00$ & NR & 30 & 0.048 & 28.00 & Saliva & {$[56]$} \\
\hline $800.00^{\mathrm{a}}$ & 0.042 & $\mathrm{LO}_{\mathrm{x}} / \mathrm{PPD} / \mathrm{PuB} / \mathrm{SPCE}$ & $0.10-1.00$ & 50.00 & NR & 0.553 & NR & Saliva & [57] \\
\hline
\end{tabular}


Table 4.2. Comparison of analytic characteristics of various lactate oxidase based-amperometric biosensors (continuation).

\begin{tabular}{|c|c|c|c|c|c|c|c|c|c|}
\hline $\begin{array}{l}\text { Enzymatic } \\
\text { units used } \\
\text { per sensor }\end{array}$ & $\begin{array}{c}\text { Working } \\
\text { potential } \\
(V)\end{array}$ & Electrode modifications & $\begin{array}{l}\text { Linearity } \\
\qquad(m M)\end{array}$ & $\begin{array}{l}L O D \\
(\mu M)\end{array}$ & $\begin{array}{c}\text { Storage } \\
\text { stability } \\
\text { (Days) }\end{array}$ & $\begin{array}{l}\text { Sensitivity } \\
\left(\mu A \boldsymbol{m M}^{-1}\right)\end{array}$ & $\begin{array}{r}R S D \\
(\%)\end{array}$ & Applications & References \\
\hline 1.00 & 0.500 & $\mathrm{LO}_{x} / \mathrm{PtNp}-\mathrm{CNF}-\mathrm{PDDA} / \mathrm{SPCE}$ & $0.03-1.50$ & 11.10 & 180 & $\begin{array}{c}36.800 \\
\mathrm{~mA} \mathrm{M}^{-1} \mathrm{~cm}^{-2}\end{array}$ & 5.80 & Blood and Sweat & [34] \\
\hline 6.40 & 0.00 & $\begin{array}{c}\mathrm{CS}-\mathrm{CNT} / \mathrm{LO}_{\mathrm{x}} / \mathrm{CS}- \\
\mathrm{CNT} / \mathrm{PuB} / \mathrm{SPAuE}\end{array}$ & $2.0-30.00$ & NR & 32 & 0.22 & 5.00 & Body sweat & [50] \\
\hline 12.12 & 0.050 & $\mathrm{CS} / \mathrm{LO}_{\mathrm{x}} / \mathrm{CMCNT} / \mathrm{TTF} / \mathrm{SPCE}$ & $10.0-20.00$ & NR & 150 & 0.644 & 3.60 & Body sweat & [74] \\
\hline 16.16 & -0.100 & $\mathrm{PVC} / \mathrm{LO}_{\mathrm{x}}-\mathrm{CS} / \mathrm{SPPuBE}$ & $0.0-28.00$ & NR & NR & 0.096 & NR & Body sweat & [53] \\
\hline
\end{tabular}

3DOM - Three-dimensional ordered microporous; ${ }^{\text {a }}$ - Units used by $\mathrm{mL}$ of a polymerization solution; CMCNT - Carboxy-functionalized multiwalled carbon nanotubes; CNF -

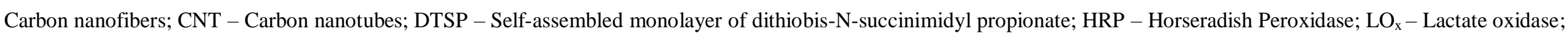
MWCNT - Multiwall carbon nanotubes; NR - not reported; PAP - poly(4-aminophenol); PuB - Prussian blue; PuBnc - Prussian blue nanocubes; PPD - poly(o-phenylenediamine); PDDA - poly(diallyldimethylammonium) chloride; PPr - poly-(phenol red); PPy - Polypyrrole; Pt - Platinum; PtNp - Platinum nanoparticles; Ptw - Platinum wire; PS Polysufone; PVC - polyvinyl chloride; SPPuBE - Screen-printed Prussian blue electrode; SPAuE - Screen-printed gold electrode; SPCE - Screen-printed Carbon electrode; TTF - tetrathiafulvalene. 


\subsubsection{Interference study}

Since the biosensor is expected to be exposed to several types of electroactive compounds, its selectivity was examined to ensure applicability in the analysis of real life samples. Therefore, potential interferences were investigated under the same experimental conditions.

First, potentially interfering substances present in wine samples were evaluated; namely malic acid, glucose, ethanol, ascorbic acid, tartaric acid, succinic acid, citric acid and acetic acid. Gallic acid, quercetin and resveratrol were also tested as possible phenolic interferents. For wine interference analysis, all evaluated compounds showed negligible electrochemical signals considering $100 \%$ the lactate response (Figure 4.10. A). In fact, as shown in figure 4.11, red wine gives an imperceptible signal when using a $\mathrm{Cu}-$ $\mathrm{MOF} / \mathrm{CS} / \mathrm{Pt} / \mathrm{SPCE}$ based sensor (without the enzyme), compared to the amperometric signal obtained with a $\mathrm{LO}_{\mathrm{x}}-\mathrm{Cu}-\mathrm{MOF} / \mathrm{CS} / \mathrm{Pt} / \mathrm{SPCE}$ based sensor.
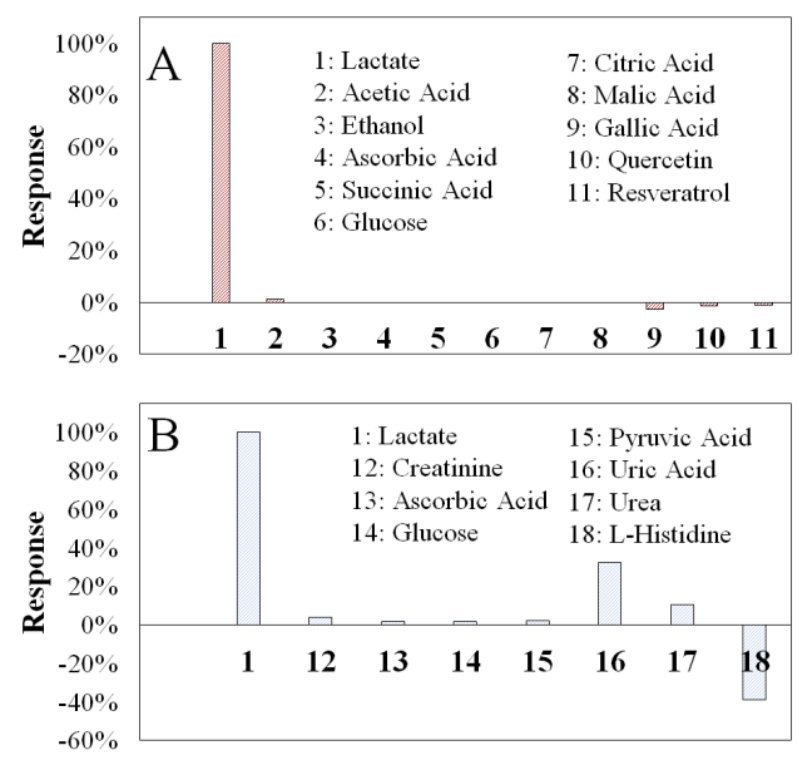

Figure 4.10. Percentages of amperometric responses of potential interferents against lactate response, at $\mathrm{LO}_{x}-\mathrm{Cu}-\mathrm{MOF} / \mathrm{CS} / \mathrm{Pt} / \mathrm{SPCE}$. $E_{a p p}: 0.15 \mathrm{~V}(\mathrm{vs} S \mathrm{SP} \mathrm{Ag} / \mathrm{AgCl})$. (A): Wine interferents. Spiked additions of $2 \mathrm{mg} \mathrm{L}^{-1}$ of each interferent compound against lactate: $0.5 \mathrm{mM}$, in phosphate buffer (0.1 M, pH 7.0); (B): Physiological interferents. Spiked additions of components at physiological levels, against $4 \mathrm{mM}$ of lactate, in phosphate buffer saline $(0.1 \mathrm{M}, \mathrm{pH} 7.0$; with $\mathrm{NaCl}$ $(23 m M))$. 


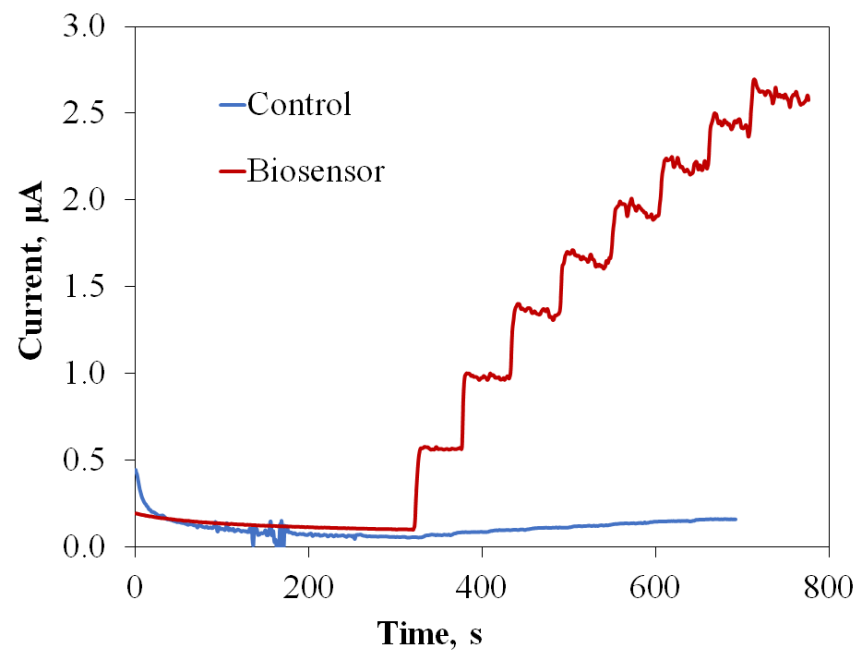

Figure 4.11. Amperograms recorded at $L O_{x}-C u-M O F / C S / P t / S P C E$ (red curve) and at control $\mathrm{Cu}$ MOF/CS/Pt/SPCE (blue curve), for the determination of lactate in wine samples. Successive additions of $10 \mu \mathrm{L}$ of red wine in phosphate buffer $\left(0.1 \mathrm{M}\right.$, pH 7.0), at $E_{a p p}$ : $0.15 \mathrm{~V}(\mathrm{vs} S P E \mathrm{Ag} / \mathrm{AgCl}$ ).

On the other hand, the plethora of metabolites and electrolytes present in perspiration and saliva potentially acting as biological interferents were considered (Figure 4.10 B). The amperometric responses of creatinine $(84 \mu \mathrm{M})$, ascorbic acid $(10 \mu \mathrm{M})$, glucose $(0.17 \mathrm{mM})$, L-histidine $(0.52 \mathrm{mM})$, pyruvic acid $(0.18 \mathrm{mM})$, uric acid $(24.5 \mu \mathrm{M})$, urea $(10 \mathrm{mM})$ and sodium chloride $(23 \mathrm{mM})$ were examined against the lactate signal in both catalysis and inhibitory concentration ranges. At physiological levels, negligible responses to creatinine, ascorbic acid, glucose and pyruvic acid, with respect to the lactate signal, were found.

Uric acid and urea were identified as interfering species, since they cause a small oxidation signal on platinum modified electrodes (Figure 4.10. B). Uric acid is present in saliva samples, however, as shown in table 3, high accuracy was achieved for lactate determination in this biological matrix analysis. Important interferences were found in the presence of L-histidine and sodium chloride (Figures 4.12 and 4.13), given that these compounds inhibit the enzyme and their presence proves to significantly affect the performance of the developed biosensor, decreasing its sensitivity. The inhibitory effect of both species was found to be greater than that caused by the lactate. As a result, the slope 
values of the inhibitory calibration curves decreased by $49 \%$ and $65 \%$ in the presence of Lhistidine and sodium chloride, respectively (Figure 4.13).

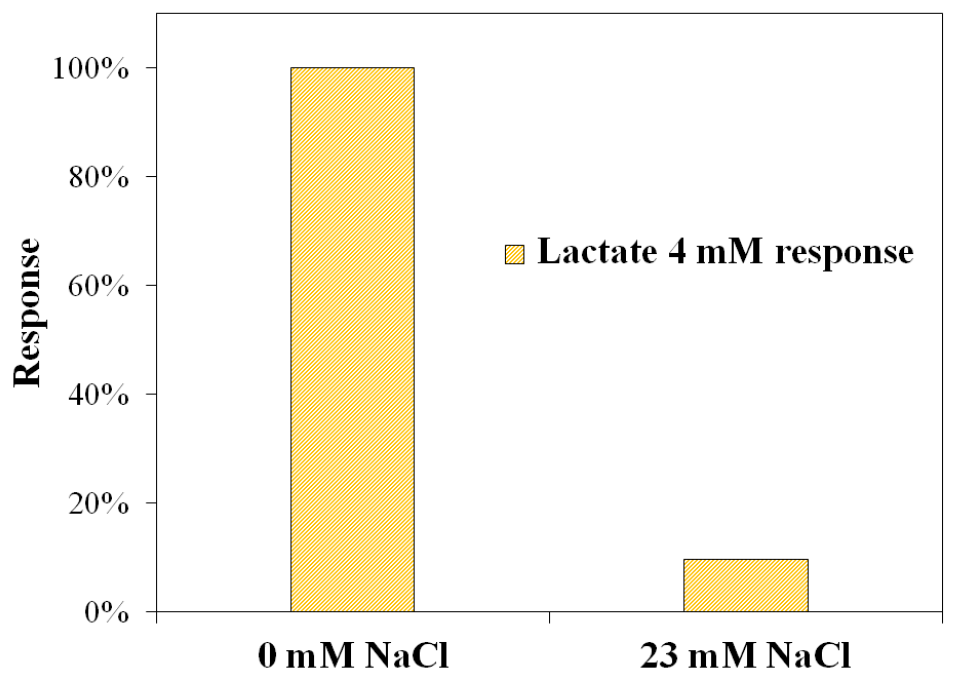

Figure 4.12. Effect of $\mathrm{NaCl}$ concentration in amperometric response for $4 \mathrm{mM}$ of lactate.

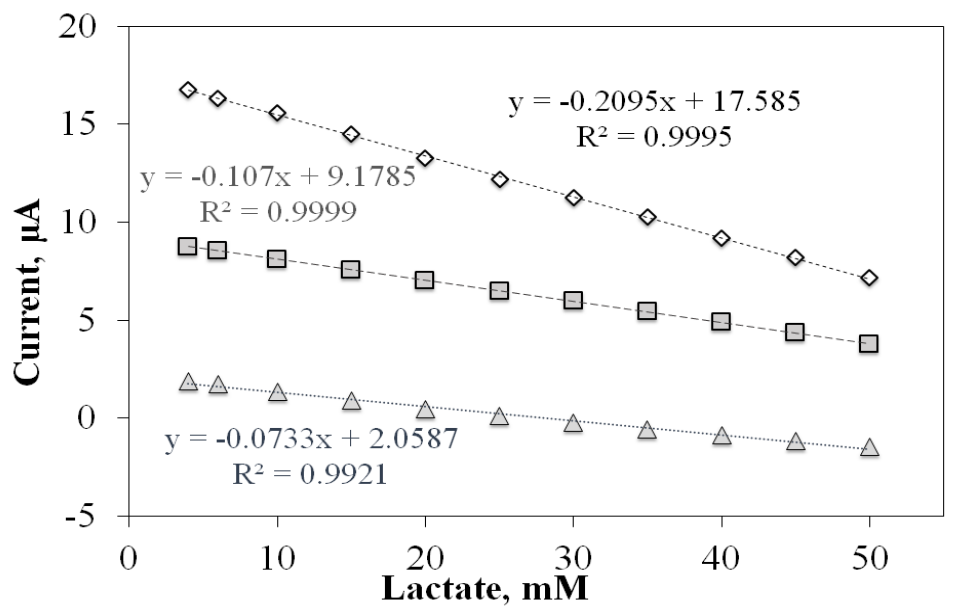

$\diamond$ [interferents] $0 \mathrm{mM} \quad \square[$ L-Hist $] 0.52 \mathrm{mM} \quad \Delta[\mathrm{NaCl}] 23 \mathrm{mM}$

Figure 4.13. Inhibitory calibration slope variation in presence of the main interferents in physiological concentrations of sweat (L-histidine and sodium chloride). 

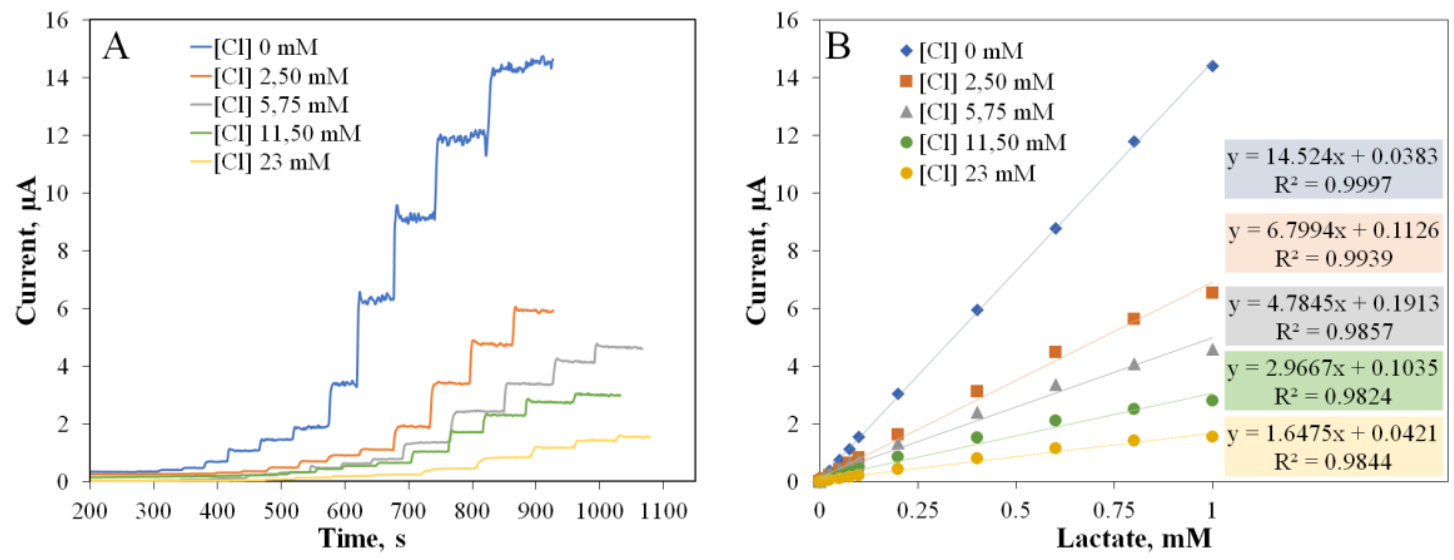

Figure 4.14. Effect of $\mathrm{NaCl}$ concentration in the slope of lactate catalysis calibration curves (from $0.75 \mu \mathrm{M}$ to $1 \mathrm{mM}$ ). (A) Amperograms recorded in increased chloride concentrations $(0.15 \mathrm{~V}$, in supporting electrolyte), and (B) calibration curves slope for the catalysis range in increased concentrations of chloride.

More than an inhibitor of the $\mathrm{LO}_{\mathrm{x}}$, chloride can be interfering with the redox reaction, since this anion is described to inhibit the $\mathrm{H}_{2} \mathrm{O}_{2}$ oxidation onto the platinum surface, as described previously by Hall [75]. Regarding this, chloride influence in the catalysis zone was checked, resulting in a loss of sensitivity dependent of the anion concentration (Figure 4.14). Notwithstanding, the developed biosensor shows that can be used to determine variations in sweat lactate concentrations during physical exercise and to assess muscular fatigue by $\mathrm{LO}_{\mathrm{x}}$ inhibition.

\subsubsection{Application in biological and food samples}

The lactate concentrations of four different samples were determined using the newly developed $\mathrm{LO}_{\mathrm{x}}-\mathrm{Cu}-\mathrm{MOF} / \mathrm{CS} / \mathrm{Pt} / \mathrm{SPCE}$ based biosensor and the results were compared with those found using HPLC analysis as the reference method. The initial concentrations of lactic acid present in the samples were determined by applying the standard addition method. Table 4.3 shows the good correlation between chromatographic and electrochemical methods for lactate quantification in different samples, evidencing that the 
proposed lactate biosensor is suitable, accurate and reliable for analyzing this type of samples.

Table 4.3. Comparison of results of lactate concentration obtained using amperometric and chromatographic methods in different samples. $n=$ number of experiments.

\begin{tabular}{cccc}
\hline Samples & $n$ & LO - Cu-MOF/CS/Pt/SPCE & HPLC \\
& & \multicolumn{2}{c}{ Lactate $(\boldsymbol{m M})$} \\
\hline Red wine & 5 & $38.82 \pm 2.20$ & $38.34 \pm 2.93$ \\
White wine & 5 & $7.90 \pm 0.39$ & $8.15 \pm 1.00$ \\
Saliva & 6 & $0.71 \pm 0.08$ & $0.73 \pm 0.01$ \\
Sweat & 6 & $34.56 \pm 1.02$ & $35.40 \pm 0.60$ \\
\hline
\end{tabular}

\subsection{CONCLUSIONS}

A new dual-range $\mathrm{LO}_{\mathrm{x}}$-based screen-printed biosensor was developed by a $\mathrm{Cu}-\mathrm{MOF}$ crosslinking of only $0.25 \mathrm{U}$ of $\mathrm{LO}_{\mathrm{x}}$ on a platinum modified surface covered with chitosan. The device proved to be suitable for a broad range of lactate determination, offering two distinct ranges of analysis: an enzymatic catalysis ranges from $0.75 \mu \mathrm{M}$ to $1 \mathrm{mM}$, and a substrate inhibitory concentrations range comprised between 4 and $50 \mathrm{mM}$. This inhibitory behavior is appropriate for determining sweat lactate as it encompasses all the possible ranges for analysis. Higher sensitivity in the modified platinum surface for lactate was found when working at $+0.15 \mathrm{~V}$ (vs SPE $\mathrm{Ag} / \mathrm{AgCl}$ ), avoiding the appearance of interferents. It has been proven that the $\mathrm{Cu}-\mathrm{MOF}$ crosslinking used with $\mathrm{CS}$ membrane result in an improved performance of the final device for both zones. The biosensor presents good accuracy, since RSD value was kept below $7 \%$ for both, catalytic and inhibitory zones. A detection limit of $0.75 \mu \mathrm{M}$ of lactate for the first zone was determined. The biosensor 
showed good repeatability for nine consecutive measurements. When stored at $4{ }^{\circ} \mathrm{C}$, biosensor long-term stability studies indicate that the sensitivity is maintained consistent over a period of 50 days.

The efficiency of the developed biosensor was tested in food samples such as white and red wines, as well as in biological samples, such as saliva and sweat. Regarding the inhibitory zone, after a convenient adaptation of the biosensor as a wearable device, could be useful in the direct measurement of high concentrations of sweat lactate. Overall, the biosensor can be potentially used to control malolactic fermentation in the winemaking process and would be highly useful for monitoring physical performance in sports medicine applications, as a noninvasive routine analysis, offering a low-cost and easy to use analysis of lactate.

\subsection{REFERENCES}

[1] Y. Fujita, K. Kawaji, A. Kanamori, K. Matoba, Y. Yajima, A. Takeuchi, K. Ishii, Relationship between age-adjusted heart rate and anaerobic threshold in estimating exercise intensity in diabetics, Diabetes Res. Clin. Pract. 8 (1990) 69-74.

[2] M.L. Goodwin, J.E. Harris, A. Hernández, L.B. Gladden, Blood lactate measurements and analysis during exercise: a guide for clinicians, J. Diabetes Sci. Technol. 1 (2007) 558-69.

[3] J. Bakker, P. Gris, M. Coffernils, R.J. Kahn, J.-L. Vincent, Serial blood lactate levels can predict the development of multiple organ failure following septic shock, Am. J. Surg. 171 (1996) 221-226.

[4] P.J. Derbyshire, H. Barr, F. Davis, S.P.J. Higson, Lactate in human sweat: A critical review of research to the present day, J. Physiol. Sci. 62 (2012) 429-440. 
[5] L.B. Gladden, Lactate metabolism: A new paradigm for the third millennium, J Physiol. 558 (2004) 5-30.

[6] S. Cairns, Lactic Acid and Exercise Performance, Sport. Med. 36 (2006) 279-291.

[7] R.A. Robergs, F. Ghiasvand, D. Parker, Biochemistry of exercise-induced metabolic acidosis, Am. J. Physiol. Regul. Integr. Comp. Physiol. 287 (2004) R502-16.

[8] G.G. Guilbault, G. Palleschi, G. Lubrano, Non-invasive biosensors in clinical analysis, Biosens. Bioelectron. 10 (1995) 379-392.

[9] E. Tékus, M. Kaj, E. Szabó, N.L. Szénási, I. Kerepesi, M. Figler, R. Gábriel, M. Wilhelm, Comparison of blood and saliva lactate level after maximum intensity exercise, Acta Biol. Hung. 63 Suppl 1 (2012) 89-98.

[10] R. Segura, C. Javierre, J.L. Ventura, M.A. Lizarraga, B. Campos, E. Garrido, A new approach to the assessment of anaerobic metabolism: measurement of lactate in saliva, $\mathrm{Br}$. J. Sports Med. 30 (1996) 305-309.

[11] L. Rassaei, W. Olthuis, S. Tsujimura, E.J.R. Sudhölter, A. Van Den Berg, Lactate biosensors: Current status and outlook, Anal. Bioanal. Chem. 406 (2014) 123-137.

[12] D.A. Sakharov, M.U. Shkurnikov, M.Y. Vagin, E.I. Yashina, A.A. Karyakin, A.G. Tonevitsky, Relationship between lactate concentrations in active muscle sweat and whole blood, Bull. Exp. Biol. Med. 150 (2010) 83-85.

[13] S. Wolfe, G. Cage, M. Epstein, L. Tice, H. Miller, R.S. Gordon, Metabolic studies of isolated human eccrine sweat glands, J. Clin. Invest. 49 (1970) 1880-1884.

[14] M.J. Buono, N.V.L. Lee, P.W. Miller, The relationship between exercise intensity and the sweat lactate excretion rate, J. Physiol. Sci. 60 (2010) 103-107.

[15] P. Pilardeau, J. Vaysse, M. Garnier, M. Joublin, L. Valeri, Secretion of eccrine sweat glands during exercise, Br. J. Sports Med. 13 (1979) 118-21. 
[16] A.B. Stefaniak, C.J. Harvey, Dissolution of materials in artificial skin surface film liquids, Toxicol. Vitr. 20 (2006) 1265-1283.

[17] C.J. Harvey, R.F. LeBouf, A.B. Stefaniak, Formulation and stability of a novel artificial sebum under conditions of storage and use, Int. J. Cosmet. Sci. 32 (2010) 347355.

[18] J.M. Green, R.C. Pritchett, T.R. Crews, J.R. McLester, D.C. Tucker, Sweat lactate response between males with high and low aerobic fitness, Eur. J. Appl. Physiol. 91 (2004) $1-6$.

[19] N. Nikolaus, B. Strehlitz, Amperometric lactate biosensors and their application in (sports) medicine, for life quality and wellbeing, Microchim. Acta. 160 (2008) 15-55.

[20] S. Pérez, E. Fàbregas, Amperometric bienzymatic biosensor for L-lactate analysis in wine and beer samples, Analyst. 137 (2012) 3854.

[21] A. Lonvaud-Funel, Lactic acid bacteria in the quality improvement and depreciation of wine, Antonie van Leeuwenhoek, Int. J. Gen. Mol. Microbiol. 76 (1999) 317-331.

[22] M. Briones, E. Casero, M.D. Petit-Domínguez, M. a. Ruiz, A.M. Parra-Alfambra, F. Pariente, E. Lorenzo, L. Vázquez, Diamond nanoparticles based biosensors for efficient glucose and lactate determination, Biosens. Bioelectron. 68 (2015) 521-528.

[23] K. Rathee, V. Dhull, R. Dhull, S. Singh, Biosensors based on electrochemical lactate detection: A comprehensive review, Biochem. Biophys. Reports. 5 (2016) 35-54.

[24] H. Teymourian, A. Salimi, R. Hallaj, Low potential detection of NADH based on Fe3O4 nanoparticles/multiwalled carbon nanotubes composite: Fabrication of integrated dehydrogenase-based lactate biosensor, Biosens. Bioelectron. 33 (2012) 60-68.

[25] C.S. Pundir, V. Narwal, B. Batra, Determination of lactic acid with special emphasis on biosensing methods: A review, Biosens. Bioelectron. 86 (2016) 777-790. 
[26] O.D. Renedo, M.A. Alonso-Lomillo, M.J.A. Martínez, Recent developments in the field of screen-printed electrodes and their related applications., Talanta. 73 (2007) 20219.

[27] H. Wu, J. Wang, X. Kang, C. Wang, D. Wang, J. Liu, I.A. Aksay, Y. Lin, Glucose biosensor based on immobilization of glucose oxidase in platinum nanoparticles/graphene/chitosan nanocomposite film, Talanta. 80 (2009) 403-406.

[28] Y.C. Tsai, S.Y. Chen, C.A. Lee, Amperometric cholesterol biosensors based on carbon nanotube-chitosan-platinum-cholesterol oxidase nanobiocomposite, Sensors Actuators, B Chem. 135 (2008) 96-101.

[29] H. Tang, J. Chen, S. Yao, L. Nie, G. Deng, Y. Kuang, Amperometric glucose biosensor based on adsorption of glucose oxidase at platinum nanoparticle-modified carbon nanotube electrode, Anal. Biochem. 331 (2004) 89-97.

[30] X. Jiang, Y. Wu, X. Mao, X. Cui, L. Zhu, Amperometric glucose biosensor based on integration of glucose oxidase with platinum nanoparticles/ordered mesoporous carbon nanocomposite, Sensors Actuators, B Chem. 153 (2011) 158-163.

[31] J. Huang, J. Li, Y. Yang, X. Wang, B. Wu, J. ichi Anzai, T. Osa, Q. Chen, Development of an amperometric L-lactate biosensor based on L-lactate oxidase immobilized through silica sol-gel film on multi-walled carbon nanotubes/platinum nanoparticle modified glassy carbon electrode, Mater. Sci. Eng. C. 28 (2008) 1070-1075.

[32] S.B. Hall, E.A. Khudaish, A.L. Hart, Electrochemical oxidation of hydrogen peroxide at platinum electrodes. Part 1. An adsorption-controlled mechanism, Electroanalysis. 43 (1998) 579-588.

[33] A. Abellán-Llobregat, I. Jeerapan, A. Bandodkar, L. Vidal, A. Canals, J. Wang, E. Morallón, A stretchable and screen-printed electrochemical sensor for glucose determination in human perspiration, Biosens. Bioelectron. 91 (2017) 885-891. 
[34] P.J. Lamas-Ardisana, O.A. Loaiza, L. Añorga, E. Jubete, M. Borghei, V. Ruiz, E. Ochoteco, G. Cabañero, H.J. Grande, Disposable amperometric biosensor based on lactate oxidase immobilised on platinum nanoparticle-decorated carbon nanofiber and poly(diallyldimethylammonium chloride) films, Biosens. Bioelectron. 56 (2014) 345-351.

[35] M. Kumar, A review of chitin and chitosan applications, React. Funct. Polym. 46 (2000) 1-27.

[36] B. Krajewska, Application of chitin- and chitosan-based materials for enzyme immobilizations: A review, Enzyme Microb. Technol. 35 (2004) 126-139.

[37] M. Rinaudo, Chitin and chitosan: Properties and applications, Prog. Polym. Sci. 31 (2006) 603-632.

[38] S. Mansouri Majd, A. Salimi, B. Astinchap, Label-free attomolar detection of lactate based on radio frequency sputtered of nickel oxide thin film field effect transistor, Biosens. Bioelectron. 92 (2017) 733-740.

[39] G. Wang, J.J. Xu, H.Y. Chen, Z.H. Lu, Amperometric hydrogen peroxide biosensor with sol-gel/chitosan network-like film as immobilization matrix, Biosens. Bioelectron. 18 (2003) 335-343.

[40] Y. Liu, M. Wang, F. Zhao, Z. Xu, S. Dong, The direct electron transfer of glucose oxidase and glucose biosensor based on carbon nanotubes/chitosan matrix, Biosens. Bioelectron. 21 (2005) 984-988.

[41] X. Kang, J. Wang, H. Wu, I.A. Aksay, J. Liu, Y. Lin, Glucose Oxidase-graphenechitosan modified electrode for direct electrochemistry and glucose sensing, Biosens. Bioelectron. 25 (2009) 901-905.

[42] G.D. Altun, S.A. Cetinus, Immobilization of pepsin on chitosan beads, Food Chem. 100 (2007) 964-971. 
[43] Ş. Akkuş Çetinus, H. Nursevin Öztop, Immobilization of catalase into chemically crosslinked chitosan beads, Enzyme Microb. Technol. 32 (2003) 889-894.

[44] J. Cruz, M. Kawasaki, W. Gorski, Electrode coatings based on chitosan scaffolds, Anal. Chem. 72 (2000) 680-686.

[45] S.E. Miller, M.H. Teplensky, P.Z. Moghadam, D. Fairen-Jimenez, Metal-organic frameworks as biosensors for luminescence-based detection and imaging, Interface Focus. 6 (2016) 20160027.

[46] H. Furukawa, K.E. Cordova, M. O’Keeffe, O.M. Yaghi, The Chemistry and Applications of Metal-Organic Frameworks, Science. 341:123044 (2013) 974.

[47] F.-X. Qin, S.-Y. Jia, F.-F. Wang, S.-H. Wu, J. Song, Y. Liu, Hemin@ metal-organic framework with peroxidase-like activity and its application to glucose detection, Catal. Sci. Technol. 3 (2013) 2761.

[48] X. Wang, X. Lu, L. Wu, J. Chen, 3D metal-organic framework as highly efficient biosensing platform for ultrasensitive and rapid detection of bisphenol A, Biosens. Bioelectron. 65 (2015) 295-301.

[49] M. Gamero, M. Sosna, F. Pariente, E. Lorenzo, P.N. Bartlett, C. Alonso, Influence of macroporous gold support and its functionalization on lactate oxidase-based biosensors response, Talanta. 94 (2012) 328-334.

[50] W. Gao, S. Emaminejad, H.Y.Y. Nyein, S. Challa, K. Chen, A. Peck, H.M. Fahad, H. Ota, H. Shiraki, D. Kiriya, D.-H. Lien, G.A. Brooks, R.W. Davis, A. Javey, Fully integrated wearable sensor arrays for multiplexed in situ perspiration analysis, Nature. 529 (2016) 509-514.

[51] M.H. Faridnia, G. Palleschi, G.J. Lubrano, G.G. Guilbault, Amperometric biosensor for determination of lactate in sweat, Anal. Chim. Acta. 278 (1993) 35-40. 
[52] W. Jia, A.J. Bandodkar, G. Valde-Ramírez, J.R. Windmiller, Z. Yang, J. Ramírez, G. Chan, J. Wang, Electrochemical Tattoo Biosensors for Real-Time Noninvasive Lactate Monitoring in Human Perspiration, Anal. Chem. 85 (2013) 6553-6560.

[53] S. Imani, A.J. Bandodkar, A.M.V. Mohan, R. Kumar, S. Yu, J. Wang, P.P. Mercier, A wearable chemical-electrophysiological hybrid biosensing system for real-time health and fitness monitoring, Nat. Commun. 7 (2016) 11650.

[54] K. Petropoulos, S. Piermarini, S. Bernardini, G. Palleschi, D. Moscone, Development of a disposable biosensor for lactate monitoring in saliva, Sensors Actuators, B Chem. 237 (2016) 8-15.

[55] J. Ballesta Claver, M.C. Valencia Mirón, L.F. Capitán-Vallvey, Disposable electrochemiluminescent biosensor for lactate determination in saliva, Analyst. 134 (2009) $1423-32$.

[56] A.M. Spehar-Délèze, S. Anastasova, P. Vadgama, Electropolymerised Phenolic Films as Internal Barriers for Oxidase Enzyme Biosensors, Electroanalysis. 26 (2014) $1335-1344$.

[57] J. Kim, G. Valdés-Ramírez, A.J. Bandodkar, W. Jia, A.G. Martinez, J. Ramírez, P. Mercier, J. Wang, Non-invasive mouthguard biosensor for continuous salivary monitoring of metabolites, Analyst. 139 (2014) 1632-6.

[58] L. V. Shkotova, T.B. Goriushkina, C. Tran-Minh, J.M. Chovelon, A.P. Soldatkin, S. V. Dzyadevych, Amperometric biosensor for lactate analysis in wine and must during fermentation, Mater. Sci. Eng. C. 28 (2008) 943-948.

[59] R. Monošík, M. Stred’anský, G. Greif, E. Šturdík, A rapid method for determination of L-lactic acid in real samples by amperometric biosensor utilizing nanocomposite, Food Control. 23 (2012) 238-244. 
[60] S. Anastasova, B. Crewther, P. Bembnowicz, V. Curto, H.M. Ip, B. Rosa, G.Z. Yang, A wearable multisensing patch for continuous sweat monitoring, Biosens. Bioelectron. 94 (2017) 730.

[61] J.Y. Lee, D.H. Olson, L. Pan, T.J. Emge, J. Li, Microporous metal-organic frameworks with high gas sorption and separation capacity, Adv. Funct. Mater. 17 (2007) $1255-1262$.

[62] M.A. Alonso-Lomillo, O. Domínguez-Renedo, L. del T. de Román, M.J. ArcosMartínez, Horseradish peroxidase-screen printed biosensors for determination of Ochratoxin A, Anal. Chim. Acta. 688 (2011) 49-53.

[63] X. Dai, R.G. Compton, Detection of As(III) via oxidation to As(V) using platinum nanoparticle modified glassy carbon electrodes: arsenic detection without interference from copper, Analyst. 131 (2006) 516-521.

[64] L. Roque, I. Escudero, J.M. Benito, Lactic acid recovery by microfiltration using niosomes as extraction agents, Sep. Purif. Technol. 151 (2015) 1-13.

[65] S. Trasatti, O.A. Petrii, Real Surface Area Measurements in Electrochemistry, J. Electroanal. Chem. 327 (1992) 353-376.

[66] J.O. Bockris, B.E. Conway, R.E. White, Modern Aspects of Electrochemistry, 1992.

[67] P. Daubinger, J. Kieninger, T. Unmüssig, G.A. Urban, Electrochemical characteristics of nanostructured platinum electrodes - a cyclic voltammetry study, Phys. Chem. Chem. Phys. 16 (2014) 8392-9.

[68] J. Wang, Analytical electrochemistry, John Wiley \& Sons, 2006.

[69] M.A. Alonso-Lomillo, O. Domínguez-Renedo, M.J. Arcos-Martinez, Enzyme modified screen printed electrodes, in: R. Courneaux, P. Novotny (Eds.), Biosens. Prop. Mater. Appl., Nova Publishers, Hauppauge NY, 2009: pp. 1-52. 
[70] J. Mocak, A. M. Bond, S. Mitchell, G. Scollary, A statistical overview of standard (IUPAC and ACS) and new procedures for determining the limits of detection and quantification: Application to voltammetric and stripping techniques (Technical Report), Pure Appl. Chem. 69 (1997) 297-328.

[71] E.R. Ziegel, Statistics and Chemometrics for Analytical Chemistry, 2004.

[72] P. Giménez-Gómez, M. Gutiérrez-Capitán, F. Capdevila, A. Puig-Pujol, C. Fernández-Sánchez, C. Jiménez-Jorquera, Monitoring of malolactic fermentation in wine using an electrochemical bienzymatic biosensor for L-lactate with long term stability, Anal. Chim. Acta. 905 (2016) 126-133.

[73] D. Jiang, Z. Chu, J. Peng, W. Jin, Screen-printed biosensor chips with Prussian blue nanocubes for the detection of physiological analytes, Sensors Actuators, B Chem. 228 (2016) 679-687.

[74] W. Jia, A.J. Bandodkar, G. Valdés-Ramírez, J.R. Windmiller, Z. Yang, J. Ramírez, G. Chan, J. Wang, Electrochemical tattoo biosensors for real-time noninvasive lactate monitoring in human perspiration, Anal. Chem. 85 (2013) 6553-6560.

[75] S.B. Hall, E.A. Khudaish, A.L. Hart, Electrochemical oxidation of hydrogen peroxide at platinum electrodes. Part V: inhibition by chloride, Electrochim. Acta. 45 (2000) 35733579 . 



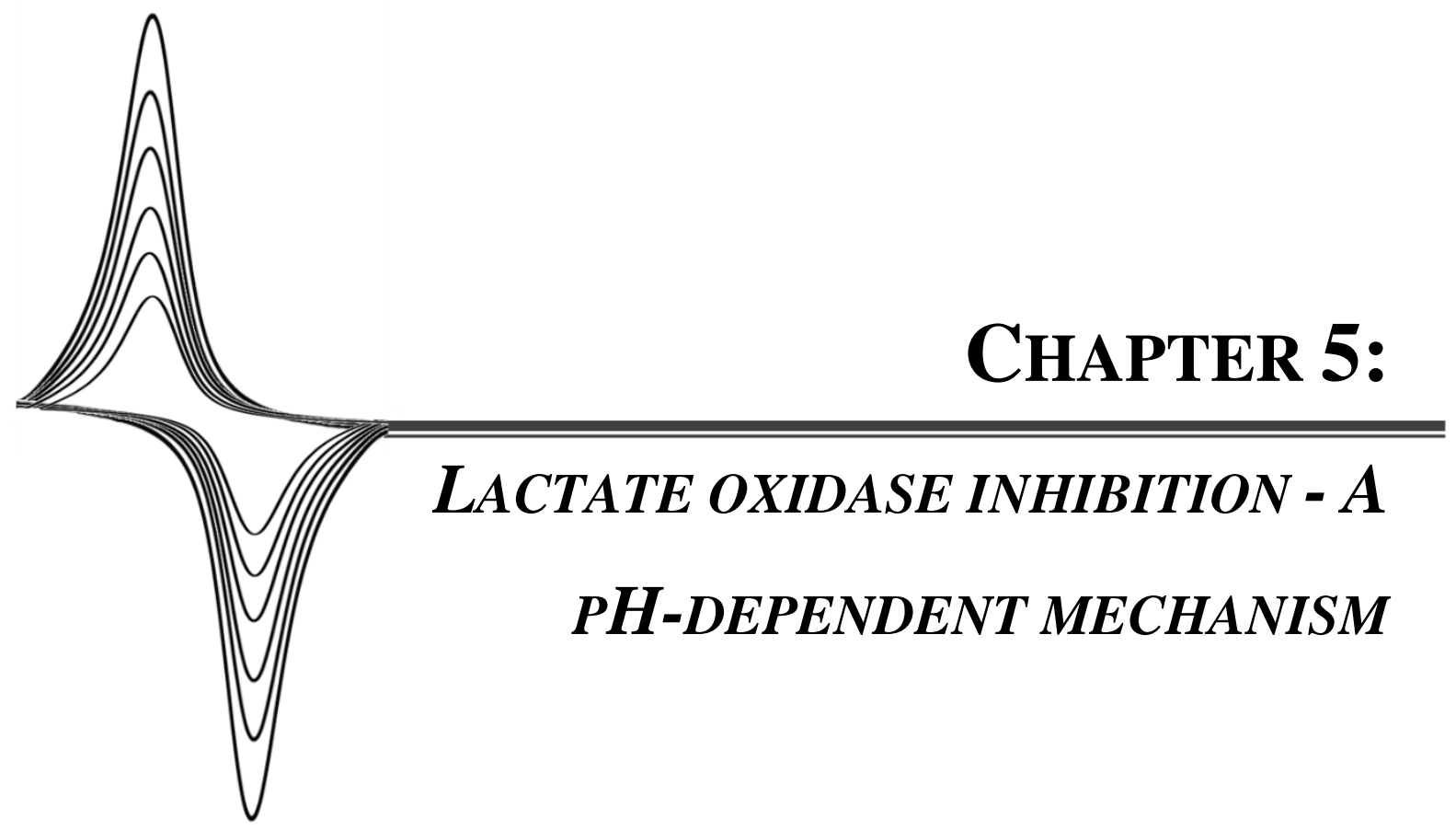



In the previous chapter, the developed biosensor shown an atypical substrate inhibition mechanism, in which $\mathrm{LO}_{\mathrm{x}}$ activity was decreased for higher lactate concentrations. Concerning this kinetic, a study regarding the alterations occurring in the $\mathrm{LO}_{\mathrm{x}}$ native structure, was carried out by fluorescence spectroscopy and dynamic light scattering (DLS). Since lactate is an organic acid and can affect the conformational state of proteins, the focus of the study relied in the search of evidences for the enzyme tertiary structure alteration, when submitted to inhibitory substrate concentrations.

Comparing the amperometric signal evolution with the results obtained by DLS and fluorescence spectroscopy, it was possible to conclude that the biosensor inhibition range, is an outcome of $\mathrm{LO}_{\mathrm{x}}$ structural alterations, that derived from a $\mathrm{pH}$-dependent mechanism promoted at high substrate concentrations.

The results described in this chapter, were achieved during a research internship financed by the Spanish Ministry of Economy and Competitiveness (EEBB-I-17-12519), at the Health Science Research Center facilities at University of Beira Interior (Covilhã Portugal), under the supervision of Professor Ana Cristina Dias Cabral.

This work is currently in the process of publication in Colloids and Surfaces B Biointerfaces. 



\subsection{INTRODUCTION}

Lactate, also known as lactic acid, is a valuable analyte with great interest in the analytical field. This organic acid is the final product of glycolysis in many organisms, being of great interest in food industry, where is used as freshness indicator for the quality and shelf-life stability of products [1]. It has been also used as acidulant and flavoring agent (E270), being of extreme importance its monitoring in the final products. Notwithstanding, the great demand for lactate analysis is found in the clinical and medical fields. As a critical metabolite of tissue oxygenation, lactate has been used as biomarker for different pathologies and as indicator for exercise prescription. Lactate is produced from the anaerobic breakdown of glucose in the tissues aiming energy production, resulting in the biochemical process known as "lactate acidosis". Whether from chronic dysfunction or excessive production, high levels of this metabolite leads to a persistent oxygen debt and to the overwhelming of body's buffering abilities $[2,3]$.

Lactate determination has been performed by means of several sensitive and accurate, but also, expensive, invasive and complicated techniques, unable to perform continuous monitoring [1]. Addressing a simple and low-cost analysis, several lactate biosensing approaches were released during the last decade [4-12]. In human body, this metabolite can be accessed through a non-invasive analysis of saliva $[5,7,8,13]$ and sweat samples $[6,12,13]$. Especially attention has been given to sweat lactate, which concentration range usually relies between $3.7 \mathrm{mM}$ and $50 \mathrm{mM}$, constituting on average $0.28 \%$ of sweat and contributing for its acidity [14].

Most of lactate biosensors are based on L-lactate oxidase enzyme ( $\mathrm{LO}_{\mathrm{x}}$ ) (E.C.1.1.3.2.), a member of the $\alpha$-hydroxyacid-oxidases flavoenzyme family. These proteins catalyze the flavin mononucleotide (FMN)-dependent oxidation of their respective substrates $[15,16]$. The enzymatic units are two tightly packed tetramers, each one forming a biologically active unit. Each monomer contains $15 \alpha$-helices, resulting in $\mathrm{LO}_{\mathrm{x}}$ subunits folded in $\alpha / \beta$ - 
barrel with two short $\beta$-strands located at the bottom of the barrel, where is located the FMN-binding site of the active center [16]. This enzyme catalyzes the lactate oxidation using molecular oxygen to generate pyruvate and $\mathrm{H}_{2} \mathrm{O}_{2}$, according to equation 3.12 (page 59) [15-17].

This flavoprotein displays a high level of substrate specificity, discriminating lactate between foreign components present in several matrices. Lactate dehydrogenase (LDH), has been also used as biorecognition element in biosensing strategies, however, the requirement of the coenzymes $\mathrm{NADH}$ or $\mathrm{NAD}^{+}$, prohibit a direct analysis of lactate, affecting the point-of-care commitment of such platforms. Also, the difficulty in immobilizing $\mathrm{LDH}$ on transducers surface, results in less selective and unstable devices [18]. These facts make of $\mathrm{LO}_{\mathrm{x}}$ a preferable enzyme for biosensing technology.

Several $\mathrm{LO}_{\mathrm{x}}$-based electrochemical biosensing platforms are described in literature [411,13]. The majority of these works use screen-printed electrodes (SPE) as transducers for biosensor fabrication, offering disposable, low-cost, easy-to-use, adaptable and point-ofcare platforms, that are able to analyze very small volumes of usually untreated samples [19].

In a recent work, our group developed a $\mathrm{LO}_{\mathrm{x}}$-based screen-printed amperometric biosensor, that was successfully applied to the lactate analysis in samples of red and white wine, sweat and saliva [13]. This electrochemical approach, allowed to register the current generated from the oxidation of the enzymatically produced $\mathrm{H}_{2} \mathrm{O}_{2}$ (equation 3.13, page 59), and correlate it with the lactate concentration present in the problem samples.

This amperometric biosensor was constructed by immobilizing the $\mathrm{LO}_{\mathrm{x}}$ enzyme through a crosslinking assisted with a glutaraldehyde (GA), copper-metallic framework (Cu-MOF) and bovine serum albumin (BSA) on a working electrode modified with Pt. The biosensor's kinetic exhibited two linear ranges of analysis: a first catalytic zone up to $1 \mathrm{mM}$ of lactate, which increased current signal reflects the catalysis described by equation 3.12 (page 59); and the second linear range, from 4 to $50 \mathrm{mM}$, that shows a decrease in current signal due to a substrate-promoted inhibition process. Similar kinetic was found for three different 
$\mathrm{LO}_{\mathrm{x}}$ types adsorbed onto working electrodes, confirming susceptibility of the biomolecule to this process [20].

In the present work, we explore the inhibition mechanism promoted by the substrate additions, considering the occurrence of conformational changes in $\mathrm{LO}_{\mathrm{x}}$ structure. The decay in fluorescence intensity, indicates that inhibitory concentrations of lactate, outcome in conformational changes of the biomolecule. Also, the slightly acidic medium obtained in the inhibition experiments, evidenced the formation of $\mathrm{LO}_{\mathrm{x}}$ aggregates by dynamic light scattering experiments. Gathering the structural data obtained for the enzyme solutions with the signal registered at the biosensor; a $\mathrm{pH}$-dependent inhibition mechanism promoted by the high concentrations of substrate, is proposed for the crosslinked $\mathrm{LO}_{\mathrm{x}}$.

\subsection{EXPERIMENTAL SECTION}

\subsubsection{Chemicals and reagents}

Lactate oxidase $\left(\mathrm{LO}_{\mathrm{x}}\right)$ from pediococcus $s p$. (E.C. 1.1.3.2.) lyophilized powder $(\geq 20$ units/mg solid), chitosan, glutaraldehyde (GA), bovine serum albumin (BSA), terephthalic acid (1,4- $\mathrm{H}_{2} \mathrm{BDC}$ ), triethylenediamine (TED) and $\mathrm{N}, \mathrm{N}$-Dimethylformamide (DMF), were

purchased from Sigma-Aldrich (Steinheim, Germany). Potassium hexachloroplatinate (IV), potassium chloride, sodium phosphate dibasic dihydrate, sodium phosphate monobasic dihydrate and potassium chloride were obtained from Merck (Darmstadt, Germany). From Panreac-Applichem (Darmstadt, Germany) were purchased L-lactic acid (85\%), sodium chloride and sodium hydroxide. Acetic acid from VWR (Briare, France).

The cooper metal framework ( $\mathrm{Cu}-\mathrm{MOF}$ ) was synthesized according to the previously reported method by [21] and [22]. Briefly, $100 \mathrm{~mL}$ of DMF were used to dissolved 0.493 $\mathrm{g}$ of copper nitrate trihydrate, $0.453 \mathrm{~g}$ of $1.4-\mathrm{H}_{2} \mathrm{BDC}$ and $0.32 \mathrm{~g}$ of TED. The mixture was sonicated to obtain a homogenous solution and heated at $120^{\circ} \mathrm{C}$ for $36 \mathrm{~h}$. The obtained blue 
crystalline power was collected and after cooling at room temperature, washed with DMF and water several times. Finally, was dried under vacuum overnight.

All the reagents used were of analytical grade and Milli-Q water (Millipore, Bedford, USA) was employed for preparing all solutions.

In the fabrication of screen-printed electrodes, the inks used, namely Electrodag PF-407 A (carbon ink), Electrodag 6037 SS (silver/silver chloride ink), Electrodag 418 (silver ink) and Electrodag 452 SS (dielectric ink), were supplied by Achenson Colloiden (Scheemda, Netherlands). Polyester films (PET) of $0.5 \mathrm{~mm}$ thickness (HIFI Industrial Film, Dardily, France), were used as the printing substrates.

\subsubsection{Screen-printed carbon electrodes (SPCE) manufacturing}

A DEK 248 screen-printing system (DEK, Weymouth, UK) was used to fabricate SPCEs [23]. Using polyester screens with the appropriate stencil, the sequential deposition of the inks was performed to define the conductive silver tracks, the $\mathrm{Ag} / \mathrm{AgCl}$ pseudo-reference electrode, the carbon counter electrode, the carbon working electrode (geometric area $0.126 \mathrm{~cm}^{2}$ ) and finally a dielectric layer.

\subsubsection{SPCE modification for lactate biosensing}

First, the carbon working electrode was modified with a Pt coating (Pt/SPCE). For that, cyclic voltammetric measurements were performed from $0.5 \mathrm{~V}$ to $-0.7 \mathrm{~V}$ (vs SPE $\mathrm{Ag} / \mathrm{AgCl}$ ), with a scan rate of $0.002 \mathrm{~V} \mathrm{~s}^{-1}$, using $200 \mu \mathrm{L}$ drop of a $1 \mathrm{mM}$ hexachloroplatinate (IV) solution in $0.1 \mathrm{M} \mathrm{KCl}$. After rinsed with Milli-Q water, the Pt modified working electrode was covered with $2 \mu \mathrm{L}$ of $0.6 \%$ chitosan (w/v) in $0.5 \%$ acetic acid and dried at room temperature (CS/Pt/SPCE). Following, the enzyme was crosslinked onto the working electrode surface, by depositing $1.20 \mu \mathrm{L}$ of a solution containing $3 \mu \mathrm{L}$ of $\mathrm{LO}_{\mathrm{x}}\left(1.5 \mathrm{U} \mu \mathrm{L}^{-1}\right.$, in $0.01 \mathrm{M}$ phosphate buffer, $\mathrm{pH} 8.0), 3 \mu \mathrm{L}$ of $\mathrm{Cu}-\mathrm{MOF}\left(2 \mathrm{mg} \mathrm{mL}^{-1}\right), 0.56 \mu \mathrm{L}$ of BSA (3\% 
$(\mathrm{w} / \mathrm{v}))$ and $0.56 \mu \mathrm{L}$ of GA $(2.5 \%(\mathrm{v} / \mathrm{v}))$. Finally, the $\mathrm{LO}_{\mathrm{x}}-$ based biosensors $\left(\mathrm{LO}_{\mathrm{x}}-\mathrm{Cu}-\right.$ $\mathrm{MOF} / \mathrm{CS} / \mathrm{Pt} / \mathrm{SPCE}$ ) were stored at $4{ }^{\circ} \mathrm{C}$ in a refrigerator until use [13].

\subsubsection{Electrochemical measurements}

A PalmSens ${ }^{\circledR}$ portable electrochemical potentiostat with the PS Trace program (PalmSens ${ }^{\circledR}$ Instruments $\mathrm{BV}$, Houten, The Netherlands) was used for chronoamperometric measurements of the biosensor modified with $\mathrm{LO}_{\mathrm{x}}$. The measurements were performed at room temperature in a cell containing $5 \mathrm{~mL}$ of supporting electrolyte, constituted by $0.1 \mathrm{M}$ phosphate buffer $(\mathrm{PB})$ at $\mathrm{pH}$ 7.0, and under constant stirring. The working electrode was operated at $+0.15 \mathrm{~V}$ (vs SPE $\mathrm{Ag} / \mathrm{AgCl}$ ), and after reaching a stable baseline, lactate concentrations were added to the electrochemical cell, from a stock solution [13].

\subsubsection{Fluorescence spectroscopy measurements}

Fluorescence spectroscopic experiments were performed on a HITACHI F-7000 fluorescence spectrophotometer (Hitachi High-Tech, Tokyo, Japan), under excitation of $\lambda_{\text {exc }}=270 \mathrm{~nm}$. Several $\mathrm{LO}_{\mathrm{x}}$ solutions of $0.5 \mu \mathrm{g} / \mu \mathrm{L}$, were prepared in $0,2,10,22.5,35$ and $47.5 \mathrm{mM}$ of lactate and left at room temperature during $30 \mathrm{~min}$. The spectra were registered with FL Solutions 4.0 Software for $\lambda_{\mathrm{em}}=280-480 \mathrm{~nm}$ and using a quartz micro cell of 500 $\mu \mathrm{L}(10 \times 2 \mathrm{~mm})$ with an optical path length of $2 \mathrm{~mm}$ (Hellma Analytics, Müllheim, Germany). The signal noise was smoothed using 3-point Savitzky-Golay function.

\subsubsection{Dynamic light scattering measurements}

Dynamic light scattering (DLS) experiments were performed on a Malvern Zetasizer NanoZS system. Disposable polystyrene size cells for aqueous samples were used to carry the enzymatic reaction at $25^{\circ} \mathrm{C}$. For that, enzymatic, lactic acid and buffer solutions were previously filtered. The reaction was carried out for $0.25 \mathrm{U}$ of $\mathrm{LO}_{\mathrm{x}}$ in $40 \mu \mathrm{L}$ of $0.1 \mathrm{M} \mathrm{PB}$ 
( $\mathrm{pH}$ 7.0), and L-lactic acid concentrations added to the cell and measured at each addition. A minimum of three measurements were made per sample.

\subsection{RESULTS AND DISCUSSION}

\subsubsection{Determination of crosslinked LOx kinetic parameters}

The reaction rate for the crosslinked $\mathrm{LO}_{\mathrm{x}}$, is measured as a result of the $\mathrm{H}_{2} \mathrm{O}_{2}$ oxidation at $+0.15 \mathrm{~V}$ (vs SPE $\mathrm{Ag} / \mathrm{AgCl}$ ). The lactate additions to the electrochemical cell leads to the generation of higher amounts of this enzymatic product, consequently increasing the current intensity output.
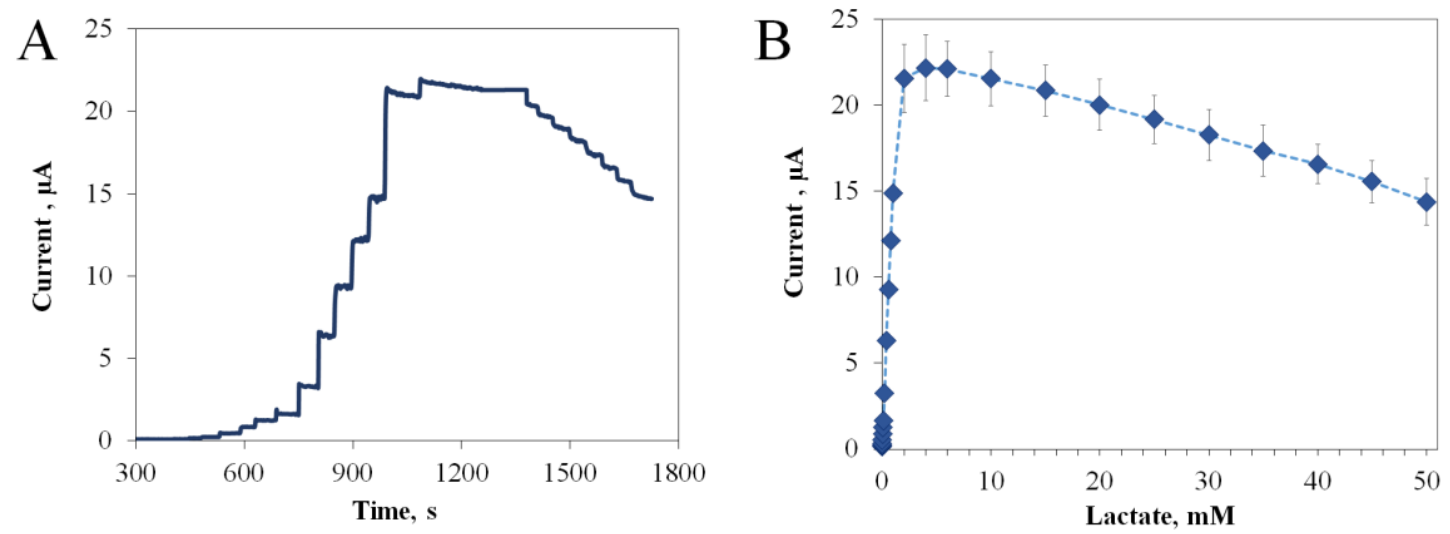

Figure 5.1. Electrochemical response of $L O_{x}-C u-M O F / C S / P t / S P C E$ biosensor. (A) Amperogram registered at $+0.15 \mathrm{~V}(\mathrm{vs} S P E \mathrm{Ag} / \mathrm{AgCl})$ for successive additions of lactate in $\mathrm{PB}(0.1 \mathrm{M}, \mathrm{pH} 7.0)$; and (B) Plotting of current vs lactate concentration $(n=5)$.

Figure 5.1 displays the typical amperogram and the response current vs substrate concentration ( $i$ vs [Lactate]), which were used to construct lactate calibration curves for the biosensing analysis. As a result of the lactate concentrations present in the 
electrochemical cell, the $\mathrm{LO}_{x}$ activity exhibits two very distinct zones. The catalytic zone results from a first-order reaction, defined between 0 and $1 \mathrm{mM}$ of lactate, in which the initial rate depends linearly on the substrate concentration. After this zone, a current output stabilization was found until $\sim 4 \mathrm{mM}$, reflecting a zero-order reaction; which is followed by a current signal decrease until $50 \mathrm{mM}$. This, second zone, reveals an inhibition phenomenon associated with the substrate, assuming a linearity applicable to samples with high content of this organic acid.

The application of kinetic models to determine the constants of immobilized bioreceptors, have been already used in biosensing field [20,24-26]. These models can be adapted for electrochemical signals, originating electrochemical equivalent equations. Regarding that, the kinetic of the crosslinked $\mathrm{LO}_{\mathrm{x}}$ for the catalytic and inhibition zones, may be represented respectively by equations 5.1 and 5.2 ,

$$
\begin{aligned}
& E+S \stackrel{K_{M}}{\longleftrightarrow} E S \rightarrow E+P \\
& E S+S \stackrel{K_{I}}{\leftrightarrows} E S S
\end{aligned}
$$

with the definitions of $K_{I}=[S][E S] /[E S S]$ and $K_{M}=[S][E] /[E S]$, the assumption of rapid equilibrium yields:

$$
i=\frac{i_{\max }[S]}{K_{M}+[S]+\frac{[S]^{2}}{K_{i}}}
$$

where, $E$ is the free enzyme, $S$ the substrate, $E S$ the enzyme-substrate complex, $P$ the reaction products and $K_{M}$ the Michaelis-Menten. ESS is the representation of an inactive complex, favored by the increment of $S$ and $K_{I}$ is the respective inhibition constant. $i$ is the steady-state current resulting from the substrate additions, $i_{\max }$ is the maximum current output registered for the saturation plateau, [S] the concentration of lactate [27]. 

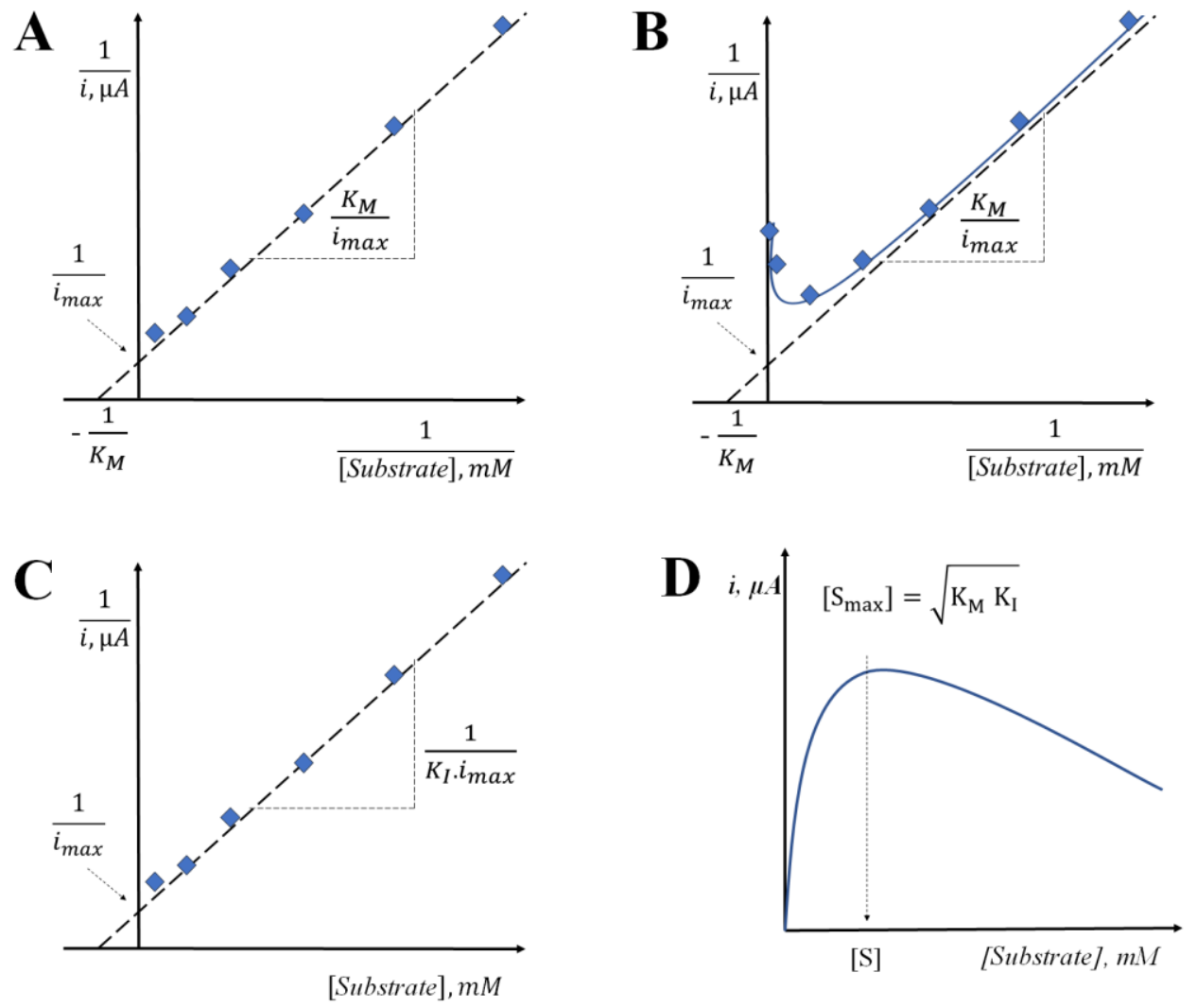

Figure 5.2. Kinetic models for amperometric biosensors. (A) Equivalent electrochemical Lineweaver-Burk model for typical catalytic process; (B) Equivalent electrochemical LineweaverBurk model for a substrate inhibition kinetic; (C) Representation of $1 / i \mathrm{vs}$ [substrate] assuming a rapid equilibrium; and (D) Equivalent electrochemical Michaelis-Menten model for a substrate inhibition kinetic.

A double-reciprocal plot describing substrate inhibition is given in Figure 5.2 - B. Thus, the biosensor kinetic parameters, such as the Michaelis-Menten constant $\left(K_{M}\right)$ for the catalytic range and the $K_{I}$ value for the atypical uncompetitive substrate inhibition, can be obtained through the biosensor response [27]. Moreover, the value of $K_{I}$ was also used to determine the value of substrate concentration, after which the inhibition behavior initiates ( $S_{\max }$, substrate concentration resulting in the maximum reaction rate).

First, the current intensity values corresponding to the first-order kinetic and saturation zones ( $0-4 \mathrm{mM}$ of lactate) were fitted to Lineweaver-Burk plot, using the electrochemical 
equivalent equation 5.4 (Figure $5.2-\mathrm{A}$ ), as under these conditions $[\mathrm{S}]^{2} / \mathrm{K}_{\mathrm{i}} \ll<1$, and inhibition effect is not observed:

$$
\frac{1}{i}=\frac{1}{i_{\max }}+\frac{K_{M}}{i_{\max }} \frac{1}{[S]}
$$

As shown in figure 5.3, the catalysis data fitted to equation $5.4(1 / i=0.0505(1 /[$ Lactate $])$ $\left.+0.0426 ; R^{2}=0.9968\right)$, resulted in the average values of $21.40 \pm 1.84 \mu \mathrm{A}$ for $i_{\max }$ and 1.05 $\pm 0.06 \mathrm{mM}$ for $K_{M}$. The $K_{M}$ value determined for the crosslinked enzyme, shows to be identical to the reported values of $0.87 \mathrm{mM}$ [28] and $0.94 \mathrm{mM}$ [17], both obtained for the free enzyme in buffered medium. Therefore, a similar intrinsic $\mathrm{LO}_{\mathrm{x}}$ activity for lactate catalysis is attained, with the additional advantage of the biomolecule stabilization, which in turn, allows to achieve a stable and a reusable device for lactate determination.

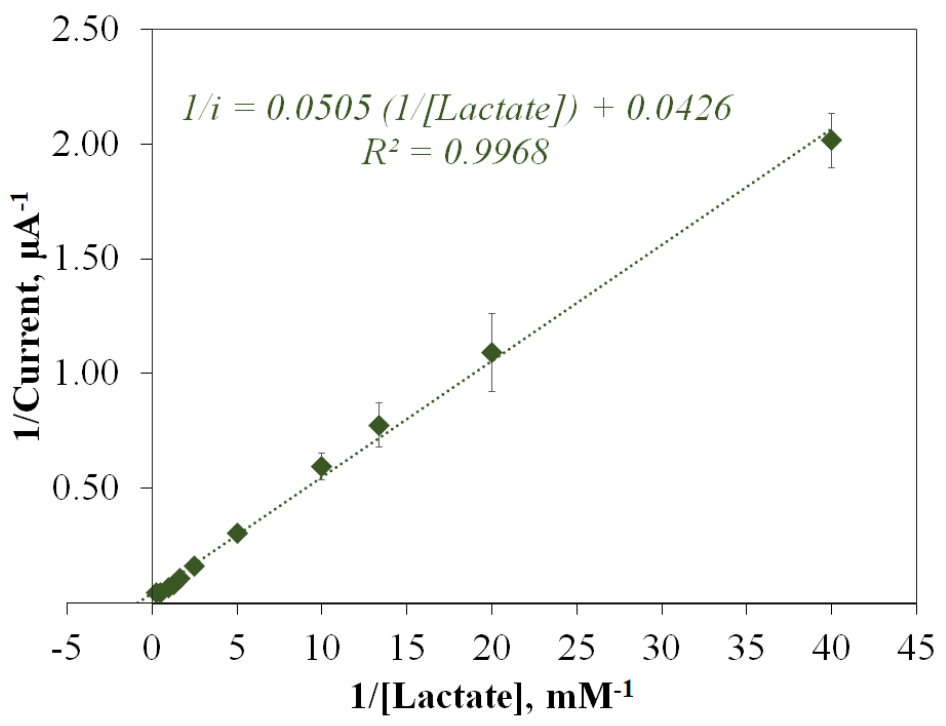

Figure 5.3. Determination of the crosslinked $L O_{x}$ kinetic constants. Lineweaver-Burk model fitting to the catalytic range, using the amperometric response for lactate additions at $0.1 \mathrm{MPB}, \mathrm{pH} 7.0$ $(n=5)$.

Secondly, the inhibition noted at high substrate concentrations, results in alteration of the enzymatic activity, imposing changes in the kinetic values. High lactate concentrations 
promote a decrease of the current signal, which is reflected in the increased significance of near zero values of the Lineweaver-Burk plot. As shown in equation 5.5, this contribution outcomes from the term $\frac{1}{i_{\max } K_{I}}$, that gain importance in definition of the Lineweaver-Burk plots at substrate inhibition mechanisms (Figure 5.2 - B) [27].

$$
\frac{1}{i}=\frac{K_{M}}{i_{\max }} \frac{1}{[S]}+\frac{1}{i_{\max } K_{I}}[S]
$$

Under these conditions, the calculation of $i_{\max }$ and $K_{I}$ values was carried out assuming a rapid equilibrium (Figure 5.4). At high lactate concentrations, these values can be determined by plotting $1 / i$ vs $[S]$, as defined in equation 5.6 (Figure $5.2-\mathrm{C}$ ) [27].

$$
\frac{1}{i}=\frac{1}{i_{\max }}+\frac{[S]}{i_{\max } K_{I}}
$$

Using the interception value obtained from the curve of $1 / i$ vs [S] (1/i $=0.0005$ [Lactate] $+0.0406 ; R^{2}=0.9911$ ), an $i_{\max }$ of $24.94 \pm 1.79 \mu \mathrm{A}$ was determined. This, is in accordance with the values observed experimentally and with the one previously determined for the catalytic and saturation zones $(21.40 \pm 1.84 \mu \mathrm{A})$; further suggesting the evolvement of a substrate inhibition mechanism.

Moreover, when the inhibition is dominant ([Lactate] $\gg K_{M}$ ), a $K_{I}$ value of $80.04 \pm 19.61$ $\mathrm{mM}$ was obtained from the curve slopes. To mention that, $\mathrm{LO}_{\mathrm{x}}$ activity is restored after each new experiment, exhibiting reversibility for the substrate inhibition process, which contributes to the reusable character of the device. Nevertheless, after ten amperometric measurements, the sensitivity for lactate determinations starts to decrease, indicating the occurrence of permanent effects [13]. Under repeated exposition to inhibitory conditions, irreversible conformational changes of the biomolecule structure, may be the cause for the consequent activity loss.

Finally, considering the previously determined kinetic parameters, it was possible to define the substrate concentration resulting in the maximum reaction rate, after which the inhibition process begins $\left(\left[S_{\max }\right]\right)$. Using equation 5.7 and setting $d i / d[S]=0$ (Figure $5.2-$ D), a $\left[S_{\max }\right]$ of $4.83 \pm 0.30 \mathrm{mM}$ was obtained [27]. 


$$
\left[S_{\text {max }}\right]=\sqrt{K_{M} K_{I}}
$$

This lactate concentration value agrees with the experimental data, where the current intensity output starts to drop at values near to $4 \mathrm{mM}$ of lactate. Thus, after a $\left[S_{\max }\right]$, the crosslinked $\mathrm{LO}_{\mathrm{x}}$ develops a biosensing response based in inhibitory kinetics. In table 5.1, are summarized the kinetic constants, determined for the crosslinked $\mathrm{LO}_{\mathrm{x}}$ at the different activity zones observed in biosensing analysis.

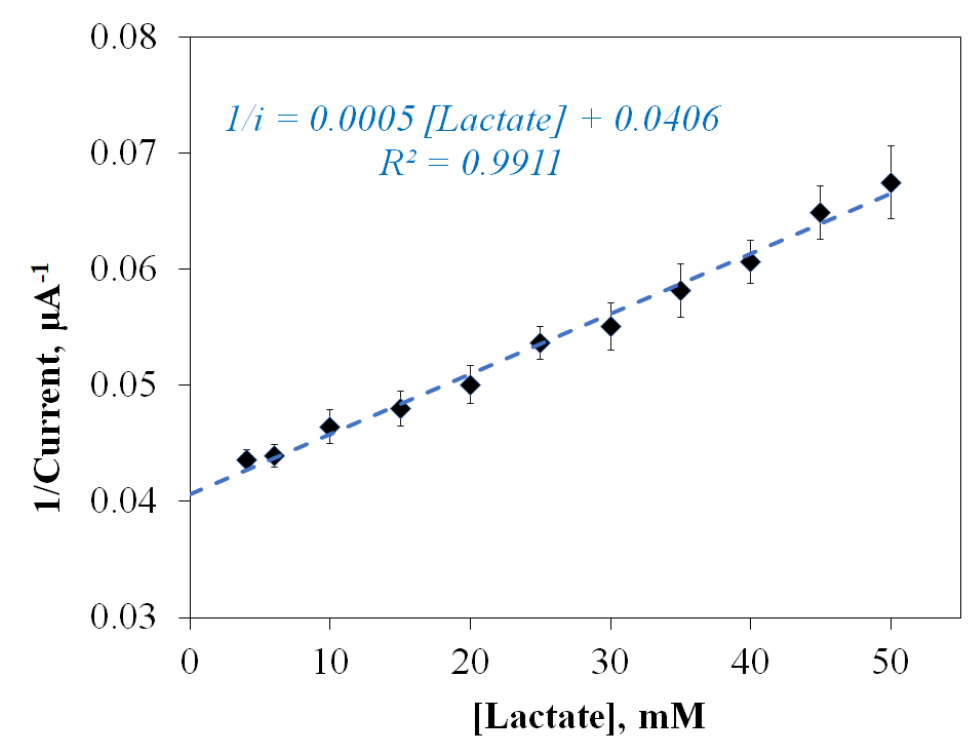

Figure 5.4. Determination of the crosslinked $L O_{x}$ kinetic parameters in inhibition range assuming a rapid equilibrium. Representation of $1 / i \mathrm{vs}[S]$, in lactate additions at $P B(0.1 \mathrm{M}, p H$ 7.0) (n=5).

Table 5.1. Kinetic parameters obtained for $\mathrm{LO}_{x}$ in normal catalysis and in the atypical substrate inhibition obtained with the biosensor $(\mathrm{n}=5)$.

\begin{tabular}{ccc}
\hline Lactate Oxidase & Catalytic Kinetic & Inhibition Kinetic \\
\hline \multirow{2}{*}{ Crosslinked } & $i_{\max }=21.40 \pm 1.84 \mu \mathrm{A}$ & $i_{\max }=24.94 \pm 1.79 \mu \mathrm{A}$ \\
& $K_{M}=1.05 \pm 0.06 \mathrm{mM}$ & $K_{I}=80.04 \pm 19.61 \mathrm{mM}$ \\
& {$\left[S_{\max }\right]=4.83 \pm 0.30 \mathrm{mM}$} \\
\hline
\end{tabular}




\subsubsection{LOx inhibition process}

Very few reports were found concerning $\mathrm{LO}_{\mathrm{x}}$ inhibition. Some anions such as chloride, sulfate, nitrate, $\alpha$-hydroymalonate and high concentrations of phosphate [29]; and also biological components such as oxalate, and hydrogencarbonate [30], 8-hydroxyquinoline, urea, ammonium, molybdate and uric acid [31], are described as foreign inhibitors of $\mathrm{LO}_{\mathrm{x}}$ activity. Moreover, atypical kinetic of inhibition was also found when lactate $[13,20]$ and 2-hydroxy-3-butynoate [32,33] were used as enzymatic substrates.

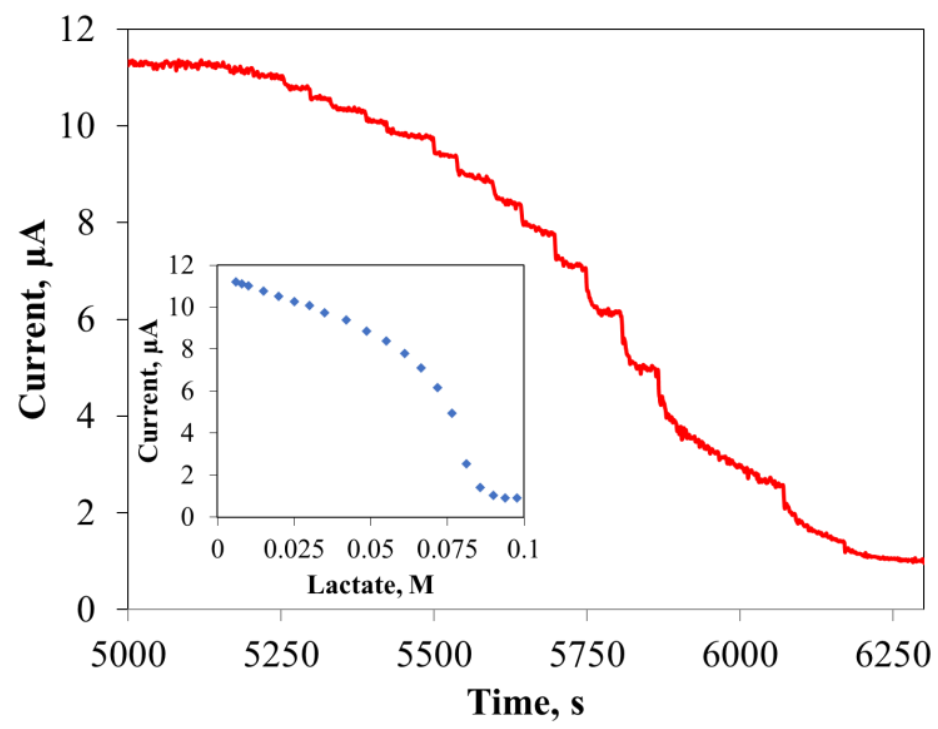

Figure 5.5. Amperogram of the full inhibitory response obtained at $L O_{x}-C u-M O F / C S / P t / S P C E$ biosensor, for lactate additions to the electrochemical cell. Inset: plotting of current vs lactate concentration as a titration-like curve.

The biosensor response, shows that the linearity observed up to $50 \mathrm{mM}$ of lactate, gives place to a titration-like curve, when this concentration value is exceeded (Figure 5.5). This may be an indicator of an altered $\mathrm{pH}$ value, which in turn, may be related with the substrate concentration added to the supporting electrolyte. Lactate is added to the electrochemical cell from lactic acid stock solutions, which is easily dissociated in lactate and protons (pKa 
3.86). Concerning this, in figure 5.6 are represented the current intensity values registered under continuous $\mathrm{pH}$ monitoring, as function of the lactate concentrations. These plots shows that the current signal for the catalytic range (from 0 to $1 \mathrm{mM}$ of lactate), increases under a stable $\mathrm{pH}$ of 7.0. At these conditions, the previously determined $K_{M}$ of $1.05 \pm 0.06$ $\mathrm{mM}$, indicates an intrinsic $\mathrm{LO}_{\mathrm{x}}$ activity. In contrast, the data obtained for saturation and inhibition zones, shows a decreased rate for lactate conversion, that can be related with the alteration of the $\mathrm{pH}$ value. The $\mathrm{pH}$ is decreased to values of 6.1 , indicating that the crosslinked $\mathrm{LO}_{\mathrm{x}}$ activity may be driven by the supporting electrolyte $\mathrm{pH}$, rather than by an atypical substrate inhibition.

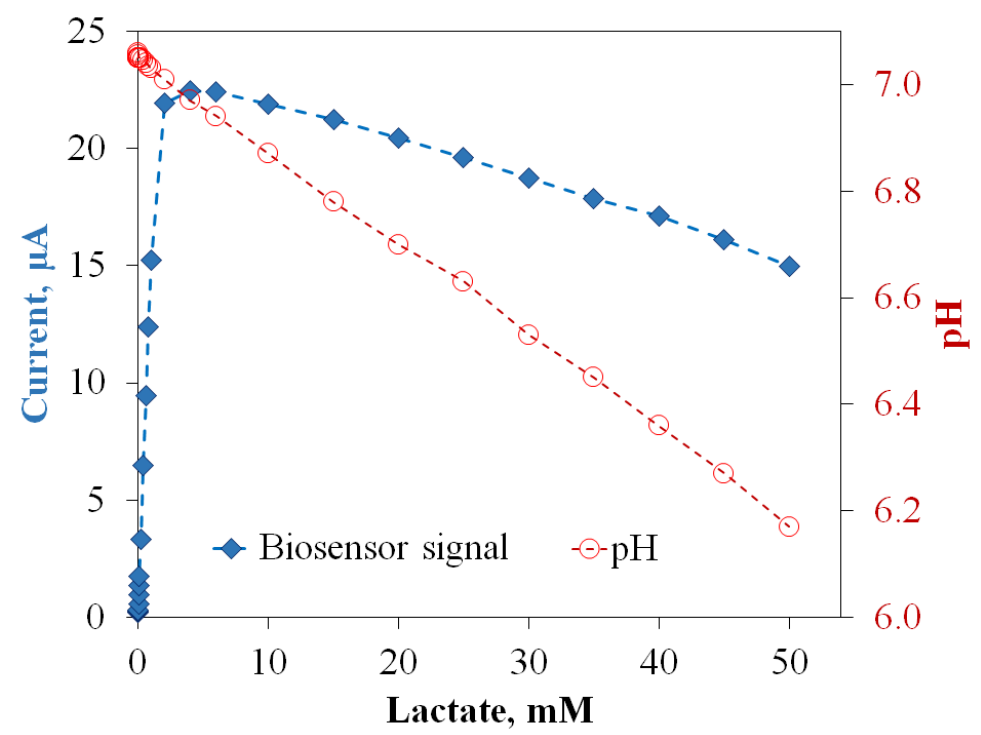

Figure 5.6. $L O_{x}-C u-M O F / C S / P t / S P C E$ biosensor kinetic registered at $+0.15 \mathrm{~V}(v s S P E \mathrm{Ag} / \mathrm{AgCl}$ ) and $\mathrm{pH}$ variation with lactate concentration in supporting electrolyte (0.1 M PB at pH 7.0).

To confirm that no additional substrate inhibition is occurring, amperometric measurements were performed using more concentrated buffer solutions, such as $0.5 \mathrm{M}$ and $1.0 \mathrm{M}$ of phosphate buffer at pH 7.0 (Figure 5.7). Since the buffering capacity of a system depends on the concentration of its components, the increased amount of the acidic and basic 
phosphate salts, should improve the $\mathrm{pH}$ stabilization during the lactic acid additions. Therefore, using these buffered solutions, the $\mathrm{LO}_{\mathrm{x}}$ catalytic activity should be isolated from the inhibition kinetic.

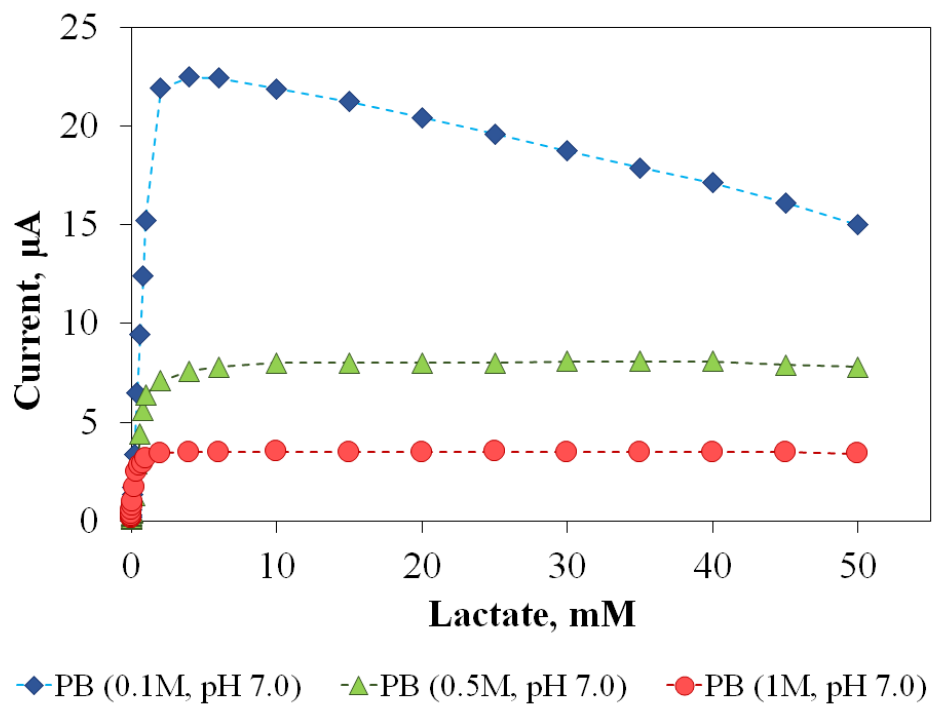

Figure 5.7. Influence of phosphate buffer concentration in the amperometric current registered at + 0.15 V(vs SPE Ag/AgCl), using the $L O_{x}-C u-M O F / C S / P t / S P C E$ biosensor.

The amperometric signals registered for the substrate additions in strong buffer solutions, exhibited lower current intensities. This seems to be related with the $\mathrm{LO}_{\mathrm{x}}$ inhibition, promoted at high concentrations of phosphate anions [29]. Despite that, it is noticeable that the biosensor response displays an extended saturation plateau, presenting stable current values until $50 \mathrm{mM}$ of lactate; and thus, avoiding the development of the inhibitory linear range. Considering that these solutions presented an increased buffer capacity, the $\mathrm{LO}_{\mathrm{x}}$ inhibitory response observed in $0.1 \mathrm{M}$ of phosphate buffer at $\mathrm{pH} 7.0$, derives from the controlled perturbation of this buffer equilibrium, allowing to obtain a quantitative relation with the substrate added to the system. This characteristic is very significant, since it can be used to perform a biosensing analysis of samples containing high lactate concentrations. Therefore, to understand the behavior of the crosslinked biocatalyst in the inhibitory 
conditions, the influence of the substrate concentrations was studied regarding the $\mathrm{LO}_{\mathrm{x}}$ structure when in solution.

\subsubsection{LOx structural changes promoted by substrate}

It is well known that the charge of the biomolecules is $\mathrm{pH}$-dependent. An inadequate $\mathrm{pH}$ value can be usually associated with the decrease of the biological activity. Since the charges plays an important role maintaining the correct conformation of the enzymes, its alteration outcomes in conformational changes of the protein structure. These conformational alterations, also affect the active center configuration, leading to the affinity loss for the substrates. Moreover, when the substrate present electric charges, its catalysis is also dependent of the active center residues charges, existing an equilibrium between $\mathrm{pH}$ value and the catalytic activity. Indeed, the majority of enzymes are recognized to have an optimal $\mathrm{pH}$ value, at which the formation of enzyme-substrate complex is favored, and the catalytic activity is maximal [27].

Some mechanisms can occur after an alteration of the protein conformational state, occasionally resulting in the formation of protein aggregates, induced by partially unfolded intermediaries in solution. Moreover, the changes in protein structure, can also result in the biomolecule adsorption to solid-liquid interfaces [34].

Considering the influence of $\mathrm{pH}$ value in proteins structure and the overall data presented, $\mathrm{LO}_{\mathrm{x}}$ solutions were studied by fluorescence spectroscopy to explore changes in the biomolecule conformational state, and the results associated with the amperometric signal registered for the crosslinked $\mathrm{LO}_{\mathrm{x}}$.

\subsubsection{Fluorescence spectroscopy}

Proteins that possess aromatic amino acid residues, such as tryptophan (Trp) and tyrosine (Tyr) can be probed using intrinsic fluorescence. Such residues are generally located within 
the hydrophobic core of the proteins, presenting a high quantum yield and, therefore, a high fluorescence intensity. In contrast, in presence of a hydrophilic environment, the fluorescence intensity for these residues is decreased. Thus, the fluorescence emission spectra of a protein, reflect changes in the environment of the aromatic amino acid residues, indicating a tertiary structure alteration due to improper folding; being consequently associated with the activity loss of the enzymes [35,36]. To inspect the inactivation behavior promoted at inhibitory concentrations of lactate, the $\mathrm{LO}_{\mathrm{x}}$ intrinsic fluorescence spectra was evaluated under increased concentrations of this substrate.

As shown in figure 5.8, the fluorescence emission spectra of $\mathrm{LO}_{\mathrm{x}}$ after excitation at a $\lambda_{\mathrm{exc}}$ $=270 \mathrm{~nm}$, presents a decreased fluorescence intensity $\left(\lambda_{\mathrm{em}}=330 \mathrm{~nm}\right)$ at higher substrate concentrations. The fluorescence recorded for the enzyme solutions in absence of lactate, was considered the one associated with the full active $\mathrm{LO}_{\mathrm{x}}$ structures, giving a fluorescence intensity value of $914.60 \pm 19.4$. Moreover, between 2 and $3.6 \mathrm{mM}$ of lactate, the $\mathrm{LO}_{\mathrm{x}}$ solutions exhibited respectively, $94.08 \%$ and $92.40 \%$ of the native structure fluorescence. Comparing this data with the signal registered at the biosensor during the saturation plateau, these still high fluorescence values, reveal an active and correct conformational state of the biocatalyst. In fact, $\mathrm{LO}_{\mathrm{x}}$ displays the maximum catalysis rate, generating the maximal current signal obtained in the biosensor analysis.

On the other hand, contrasting information was found for the fluorescence data in the inhibitory lactate levels. In presence of lactate at $10 \mathrm{mM}$, a fluorescence intensity value of $759.9 \pm 25.70$ was observed, evidencing the presence of biomolecules with about $20 \%$ less fluorescence. Thus, the slightly acidic conditions reached in presence of high substrate concentrations, leads to alterations on the enzymatic core environment, and consequently, result in changes of the biomolecule conformational state. Furthermore, regarding the amperometric response registered for $10 \mathrm{mM}$ of lactate, it is possible to associate the decreased enzymatic activity with the conformational changes detected in fluorescence experiments. 

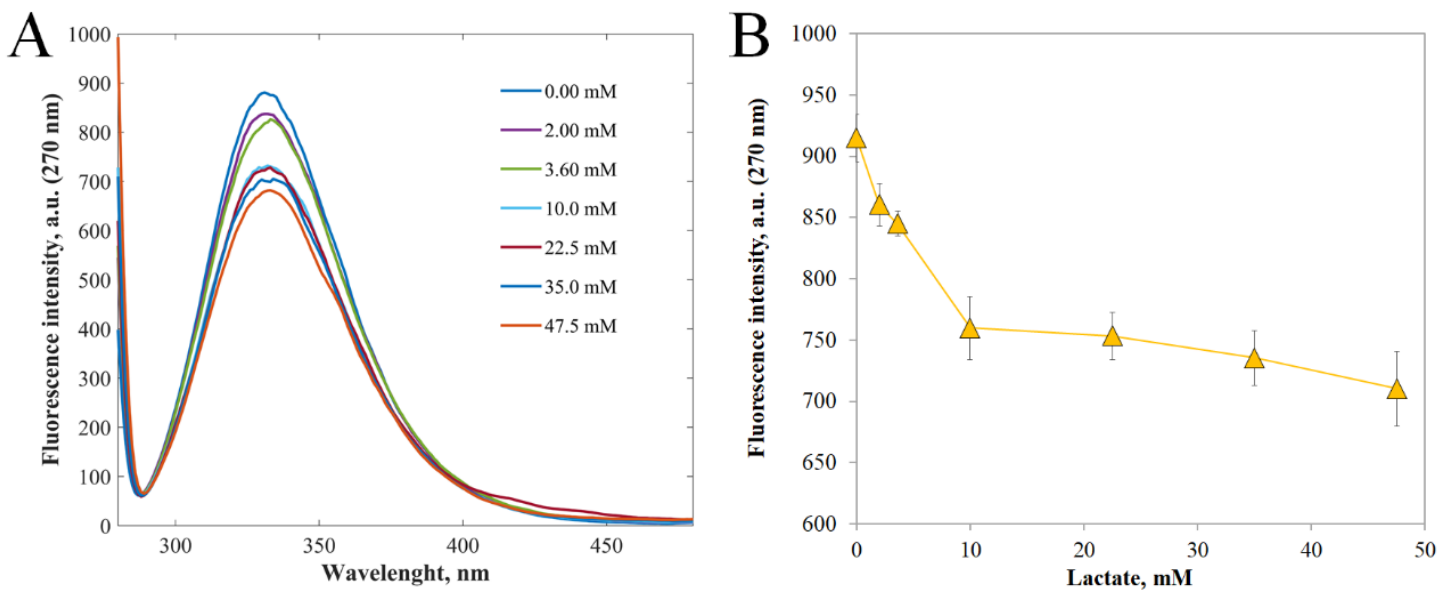

Figure 5.8. Fluorescence spectroscopy for $L O_{x}$ solutions. (A) Fluorescence spectra of $L O_{x}(0.5$ $\mu \mathrm{g} / \mu \mathrm{L})$ in 0.1 M PB (pH 7.0), containing $0,2,3.6,10,22.5,35$ and $47.5 \mathrm{mM}$ of lactate $\left(\lambda_{\text {exc }}=270\right.$ $n m) ;(B) L O_{x}$ conformational changes monitored by collecting the fluorescence intensity at $330 \mathrm{~nm}$ for increased lactate concentrations $(n=3)$.

Lactate concentrations higher than $10 \mathrm{mM}$, present a slower decay of the fluorescence values of the $\mathrm{LO}_{\mathrm{x}}$ solutions; however, displaying a strong inhibition in the amperometric response. This indicate that, after this point, different processes are occurring at the $\mathrm{LO}_{\mathrm{x}}$ structural conformation. Therefore, there is the possibility that the immobilized enzyme is undergoing conformational changes at the electrode surface, resulting in the inhibitory zone observed. The nearly steady-state of fluorescence observed after $10 \mathrm{mM}$, specify that the surrounding environment of the aromatic aminoacidic residues does not undergo in extensive modifications. Accordingly, these data point to the formation of aggregates, in which the hydrophobic residues are usually present in the interior of these structures. Thus, dynamic light scattering (DLS) of $\mathrm{LO}_{\mathrm{x}}$ solutions was used to evaluate and identify the formation of protein aggregates in inhibitory conditions. 


\subsubsection{Dynamic light scattering}

Dynamic light scattering (DLS) is a well-established and widely used tool, to evaluate hydrodynamic size and size distribution of several type of particles such as proteins, protein aggregates, polymers or nanoparticles. Based on the scattering of light from particles and their brownian motion, this technique allows to determine a broad range of hydrodynamic sizes [37]. The destabilization and unfolding of proteins, typically result in the displaying of aminoacidic residues that create nucleation points for aggregates growth. These aggregates generally show a lack of catalytic activity and can be formed from a combination of hydrophobic interactions, hydrogens bonds and/or Van der Waals forces [34], all favored with changes of the medium $\mathrm{pH}$. Accordingly, it is possible to identify such aggregates through the increase of the hydrodynamic size of species in solution (Figure 5.9).

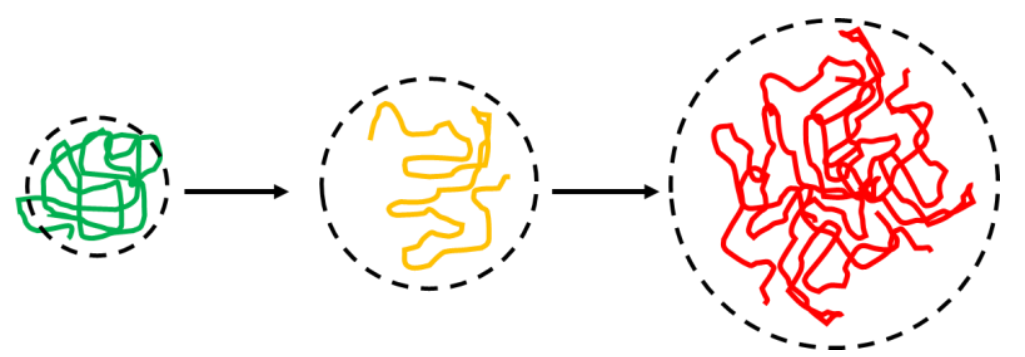

Figure 5.9. DLS fundamentals of protein aggregation. Dotted circle represents the hydrodynamic size.

DLS experiments were carried out in $\mathrm{LO}_{\mathrm{x}}$ and $0.1 \mathrm{M} \mathrm{PB}(\mathrm{pH}$ 7.0) solutions, and the size distribution evaluated under different lactate concentrations. In absence of substrate, it was found a monodisperse solution; however, the polydispersity starts to be evident when lactate is added to the medium. This polydispersity is associated with the presence of a nonpure hydrodynamic size distribution, therefore, indicating the presence of different sizes in solution. 
Relying in distribution, the DLS data were collected into three main hydrodynamic size families: the smallest size of $8.99 \pm 0.55 \mathrm{~nm}$ (Family 1); an intermediary size of $56.64 \pm$ $12.43 \mathrm{~nm}$ (Family 2), and finally, with a size of $263.63 \pm 23.90 \mathrm{~nm}$ the larger particles (Family 3). This size distribution can be related with the different conformations of the $\mathrm{LO}_{\mathrm{x}}$, as a result of the alteration in solution properties during lactate additions.

Estimated with a molecular weight of $80 \mathrm{kDa}$, the smallest particles of family 1 are representative of non-aggregated and full active $\mathrm{LO}_{\mathrm{x}}$ structures, which displays the same molecular weight in gel filtration. As can be seen in figure 5.10, the presence of this hydrodynamic size prevails between 0 and $3.56 \mathrm{mM}$ of lactate, after which the percentage starts to decrease, marking the beginning of the inhibition zone observed for the crosslinked enzyme. The homogeneous distribution of the family 1 during the catalytic and saturation zones, shows that the major contribution for the electrochemical signal generation, is due to the native structures of $\mathrm{LO}_{\mathrm{x}}$, which offer intrinsic activity for lactate conversion.

Despite the prevalence of these active structures during the catalytic and saturation zones, the presence of intermediary species (Family 2), is noticeable since the beginning of substrate additions. The size distribution of family 2 , remain lower than $0.2 \%$ during the catalytic and saturation zones of the amperometric response, contributing to the polydispersity of the solution. The presence of this family shows insignificant effects on the global activity registered in the catalytic and saturation zones for the crosslinked $\mathrm{LO}_{\mathrm{x}}$.

The homogeneity of size distribution suffers a drastic change in the lactate range, from 3.6 and $22.5 \mathrm{mM}$. For $9.9 \mathrm{mM}$ of lactate, the family 1 distribution drops to $98.8 \%$, the family 2 is kept at $0.2 \%$; and a new family 3 of larger hydrodynamic sizes appears in solution. The presence of the family 3 confirm, that the native structures of $\mathrm{LO}_{\mathrm{x}}$ are aggregating due to the destabilization of the medium $\mathrm{pH}$. At $22.5 \mathrm{mM}$ of lactate, the solutions' size distribution is completely changed, with only $34 \%$ of family 1 , also showing an increased presence of family 2 intermediary species $(65 \%)$ and nearly $0.5 \%$ of family 3 prevalence. The family 2, with the intermediary structures, plays an important role in the aggregation mechanism, acting like a trigger for the formation of larger and inactive protein aggregates. At this point, the decrease in amperometric signal, can be associated with the low availability of 
active and non-aggregated $\mathrm{LO}_{\mathrm{x}}$ structures that, in solution, have been mainly converted into larger and inactive aggregates. Indeed, at higher substrate concentrations, the family 3 prevails as the main species in solution. DLS experiments, also indicate that the native $\mathrm{LO}_{\mathrm{x}}$ structures and the intermediary species of family 2 , are totally depleted for the highest substrate concentrations testes. In fact, a monodisperse solution of larger $\mathrm{LO}_{\mathrm{x}}$ aggregates is noticed at $47.3 \mathrm{mM}$.

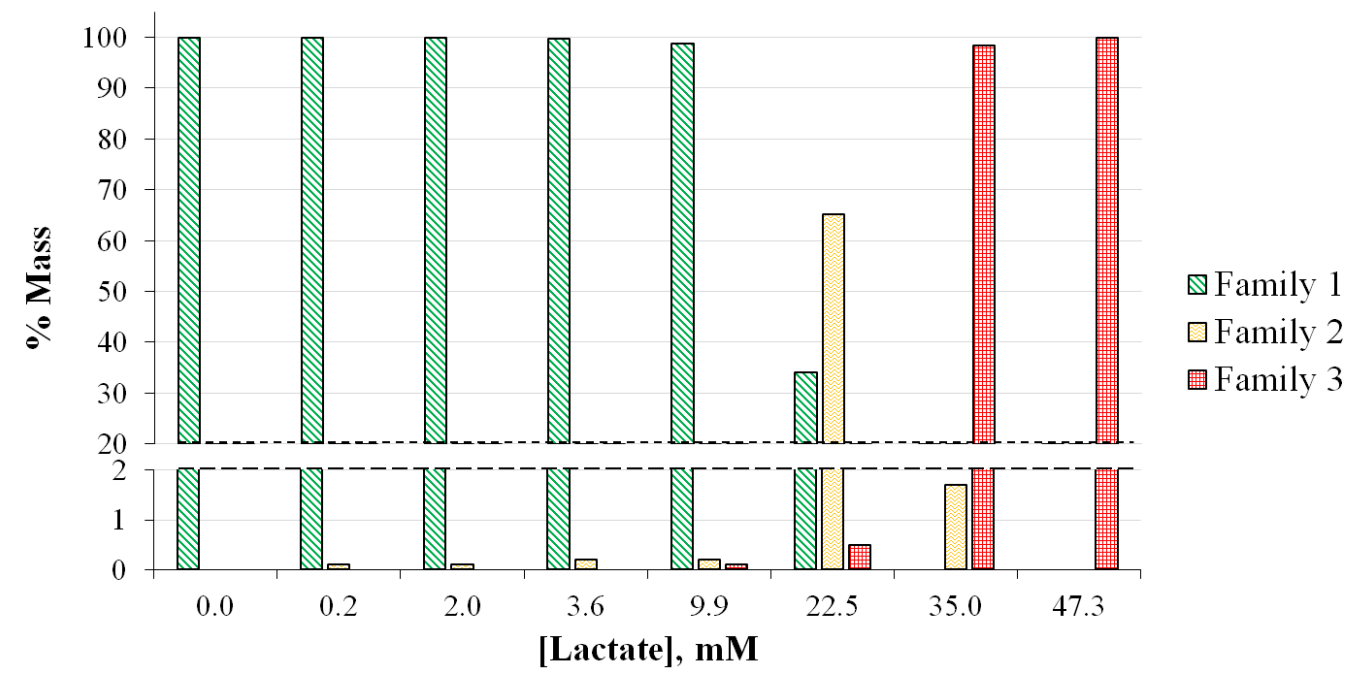

Figure 5.10. DLS analysis. Hydrodynamic size distribution by volume for $L O_{x}$ solutions in presence of different lactate concentrations (0.1 M PB, pH 7.0). Data obtained from the distribution results.

Gathering the DLS and amperometric results with the fluorescence data, it is possible to associate the fluorescence intensity decay, observed between 10 and $47.5 \mathrm{mM}$ of lactate, with the formation of the larger $\mathrm{LO}_{\mathrm{x}}$ sizes from family 3 (Figure 5.10). These aggregates internalize the hydrophobic residues, stabilizing the fluorescence intensity values. Moreover, concerning this effect, the inhibited enzymatic reaction of crosslinked $\mathrm{LO}_{\mathrm{x}}$, reflect conformational changes on the tertiary structure of the immobilized protein, that present a decreased affinity to the substrate, due to a $\mathrm{pH}$-dependent mechanism. 


\subsection{CONCLuSions}

The results obtained in this work explore the substrate inhibition mechanism detected for the crosslinked $\mathrm{LO}_{\mathrm{x}}$. Conformational alterations of the enzyme structure in solution were accompanied during the course of the catalytic and inhibitory reactions.

Throughout the analysis performed with the biosensor, it was found that substrate additions to the electrochemical cell, resulted in slightly acidic supporting electrolyte. The change in the supporting electrolyte buffering equilibrium, affected the crosslinked $\mathrm{LO}_{\mathrm{x}}$ activity, giving place to lower rates for substrate catalysis. The use of concentrated phosphate buffer solutions as supporting electrolyte in amperometric measurements, allowed to observe an enzymatic saturation extended up to $50 \mathrm{mM}$, demonstrating that such inhibition mechanism is related with a $\mathrm{pH}$ variation, rather than with a substrate inhibition.

The decay in $\mathrm{LO}_{\mathrm{x}}$ intrinsic fluorescence intensity, evidenced conformational alterations on the enzyme tertiary structure, with dependence of substrate concentrations. Moreover, the presence of a fluorescence steady-state for inhibitory zone, indicates the internalization of hydrophobic residues into different protein structures. Moreover, the DLS data, shows that the hydrodynamic size detected in $\mathrm{LO}_{\mathrm{x}}$ solutions, is also highly dependent of lactate concentrations. For the catalytic range $(0-1 \mathrm{mM}$ of lactate), the enzymatic solutions evidenced the native form of $\mathrm{LO}_{\mathrm{x}}$. However, during the saturation zone the availability of non-aggregated $\mathrm{LO}_{\mathrm{x}}$ structures starts to drop, giving place to intermediary species. These intermediaries, were noted as transitional stages for the nucleation into larger aggregates, demonstrating its importance in the aggregation mechanism in solution. In fact, substrate concentrations that exhibit a stronger inhibition, resulted in the formation of larger and inactive $\mathrm{LO}_{\mathrm{x}}$ aggregates, which constitute $100 \%$ of the solution hydrodynamic size distribution. 
Accordingly, it is possible to establish a relation between the conformational changes and the consequent aggregation of the enzyme in solution, with the supporting electrolyte $\mathrm{pH}$, perturbed by the substrate additions. Therefore, these factors are the main contributors in the crosslinked $\mathrm{LO}_{\mathrm{x}}$ activity loss, and consequently, the main cause for inhibitory range observed in the biosensing approach.

\subsection{REFERENCES}

[1] K. Rathee, V. Dhull, R. Dhull, S. Singh, Biosensors based on electrochemical lactate detection: A comprehensive review, Biochem. Biophys. Reports. 5 (2016) 35-54.

[2] L.B. Gladden, Lactate metabolism: A new paradigm for the third millennium, J Physiol. 558 (2004) 5-30.

[3] N. Nikolaus, B. Strehlitz, Amperometric lactate biosensors and their application in (sports) medicine, for life quality and wellbeing, Microchim. Acta. 160 (2008) 15-55.

[4] P. Giménez-Gómez, M. Gutiérrez-Capitán, F. Capdevila, A. Puig-Pujol, C. Fernández-Sánchez, C. Jiménez-Jorquera, Monitoring of malolactic fermentation in wine using an electrochemical bienzymatic biosensor for L-lactate with long term stability, Anal. Chim. Acta. 905 (2016) 126-133.

[5] J. Kim, G. Valdés-Ramírez, A.J. Bandodkar, W. Jia, A.G. Martinez, J. Ramírez, P. Mercier, J. Wang, Non-invasive mouthguard biosensor for continuous salivary monitoring of metabolites, Analyst. 139 (2014) 1632-6.

[6] W. Gao, S. Emaminejad, H.Y.Y. Nyein, S. Challa, K. Chen, A. Peck, H.M. Fahad, H. Ota, H. Shiraki, D. Kiriya, D.-H. Lien, G.A. Brooks, R.W. Davis, A. Javey, Fully integrated wearable sensor arrays for multiplexed in situ perspiration analysis, Nature. 529 (2016) 509-514. 
[7] J. Ballesta Claver, M.C. Valencia Mirón, L.F. Capitán-Vallvey, Disposable electrochemiluminescent biosensor for lactate determination in saliva, Analyst. 134 (2009) $1423-32$.

[8] K. Petropoulos, S. Piermarini, S. Bernardini, G. Palleschi, D. Moscone, Development of a disposable biosensor for lactate monitoring in saliva, Sensors Actuators, B Chem. 237 (2016) 8-15.

[9] R. Monošík, M. Stred'anský, G. Greif, E. Šturdík, A rapid method for determination of L-lactic acid in real samples by amperometric biosensor utilizing nanocomposite, Food Control. 23 (2012) 238-244.

[10] S. Anastasova, B. Crewther, P. Bembnowicz, V. Curto, H.M. Ip, B. Rosa, G.Z. Yang, A wearable multisensing patch for continuous sweat monitoring, Biosens. Bioelectron. 94 (2017) 730 .

[11] I. S. Kucherenko, Ya. V. Topolnikova, O. O. Soldatkin, Advances in the biosensors for lactate and pyruvate detection for medical applications: a review, TrAC Trends Anal. Chem. 110 (2018) 160-172.

[12] A. Abellán-Llobregat, I. Jeerapan, A. Bandodkar, L. Vidal, A. Canals, J. Wang, E. Morallón, A stretchable and screen-printed electrochemical sensor for glucose determination in human perspiration, Biosens. Bioelectron. 91 (2017) 885-891.

[13] H. Cunha-Silva, M.J. Arcos-Martinez, Dual range lactate oxidase-based screen printed amperometric biosensor for analysis of lactate in diversified samples, Talanta. 188 (2018) 779-787.

[14] A.B. Stefaniak, C.J. Harvey, Dissolution of materials in artificial skin surface film liquids, Toxicol. Vitr. 20 (2006) 1265-1283.

[15] Y. Umena, K. Yorita, T. Matsuoka, A. Kita, K. Fukui, Y. Morimoto, The crystal structure of L-lactate oxidase from Aerococcus viridans at $2.1 \AA$ resolution reveals the mechanism of strict substrate recognition, Biochem. Biophys. Res. Commun. 350 (2006) 249-256. 
[16] I. Leiros, E. Wang, T. Rasmussen, E. Oksanen, H. Repo, S.B. Petersen, P. Heikinheimo, E. Hough, The 2.1 $\AA$ structure of Aerococcus viridans L-lactate oxidase (LOX), Acta Crystallogr. Sect. F Struct. Biol. Cryst. Commun. 62 (2006) 1185-1190.

[17] K. Maeda-Yorita, K. Aki, H. Sagaib, H. Misakib, V. Massey, L-Lactate oxidase and L-lactate monooxygenase: Mechanistic variations on a common structural theme, Biochimie. 77 (1995) 631-642.

[18] C.S. Pundir, V. Narwal, B. Batra, Determination of lactic acid with special emphasis on biosensing methods: A review, Biosens. Bioelectron. 86 (2016) 777-790.

[19] O.D. Renedo, M.A. Alonso-Lomillo, M.J.A. Martínez, Recent developments in the field of screen-printed electrodes and their related applications., Talanta. 73 (2007) 20219.

[20] I. Taurino, R. Reiss, M. Richter, M. Fairhead, L. Thöny-Meyer, G. De Micheli, S. Carrara, Comparative study of three lactate oxidases from Aerococcus viridans for biosensing applications, Electrochim. Acta. 93 (2013) 72-79.

[21] J.Y. Lee, D.H. Olson, L. Pan, T.J. Emge, J. Li, Microporous metal-organic frameworks with high gas sorption and separation capacity, Adv. Funct. Mater. 17 (2007) $1255-1262$.

[22] X. Wang, X. Lu, L. Wu, J. Chen, 3D metal-organic framework as highly efficient biosensing platform for ultrasensitive and rapid detection of bisphenol A, Biosens. Bioelectron. 65 (2015) 295-301.

[23] B. Molinero-Abad, D. Izquierdo, L. Pérez, I. Escudero, M.J. Arcos-Martínez, Comparison of backing materials of screen printed electrochemical sensors for direct determination of the sub-nanomolar concentration of lead in seawater, Talanta. 182 (2018) $549-557$.

[24] R. Baronas, J. Kulys, A. Lančinskas, A. Žilinskas, Effect of diffusion limitations on multianalyte determination from biased biosensor response, Sensors (Switzerland). 14 (2014) 4634-4656. 
[25] J. Kulys, Biosensor Response at Mixed Enzyme Kinetics and External Diffusion Limitation in Case of Substrate Inhibition, Nonlinear Anal. Model. Control. 11 (2006) 385392.

[26] D. Simelevicius, R. Baronas, Mechanisms controlling the sensitivity of amperometric biosensors in flow injection analysis systems, J. Math. Chem. 49 (2011) 1521-1534.

[27] M.L. Shuler, F. Kargi, Enzymes, in: Bioprocess Eng. - Basic Concepts, 2nd ed., Upper Saddle River, NJ : Prentice Hall, 2002: pp. 57-97.

[28] K. Yorita, K. Aki, T. Ohkuma-Soyejima, T. Kokubo, H. Misaki, V. Massey, Conversion of L-lactate oxidase to a long chain $\alpha$-hydroxyacid oxidase by site-directed mutagenesis of alanine 95 to glycine, J. Biol. Chem. 271 (1996) 28300-28305.

[29] O. Lockridge, V. Massey, P.A. Sullivan, Mechanism of action in the flavoenyzme lactate oxidase, J. Biol. Chem. 247 (1972) 8097-8106.

[30] M. Mascini, D. Moscone, G. Palleschi, A lactate electrode with lactate oxidase immobilized on nylon net for blood serum samples in flow systems, Anal. Chim. Acta. 157 (1984) 45-51.

[31] S. Suman, R. Singhal, A.L. Sharma, B.D. Malthotra, C.S. Pundir, Development of a lactate biosensor based on conducting copolymer bound lactate oxidase, Sensors Actuators, B Chem. 107 (2005) 768-772.

[32] C.T. Walsh, A. Schonbrunn, O. Lockridge, V. Massey, R. H. Abeles, Inactivation of a Flavoprotein, Lactate Oxidase, by Acetylenic Substrate, J. Biol. Chem. 247 (1972) 60046006.

[33] S. Ghisla, V. Massey, R.H. Abeles, C.T. Walsh, H. Ogata, A. Schonbrunn, Kinetic Studies on the Inactivation of L-Lactate Oxidase by [the Acetylenic Suicide Substrate] 2Hydroxy-3-Butynoate, Biochemistry. 15 (1976) 1791-1797.

[34] W. Wang, C.J. Roberts, Protein aggregation - Mechanisms, detection, and control, Int. J. Pharm. 550 (2018) 251-268. 
[35] M.R. Eftink, Fluorescence Techniques for Studying Protein Structure, 1991.

[36] B. Manta, G. Obal, A. Ricciardi, O. Pritsch, A. Denicola, Tools to evaluate the conformation of protein products, Biotechnol. J. 6 (2011) 731-741.

[37] A. Khodabandehloo, D.D.Y. Chen, Particle sizing methods for the detection of protein aggregates in biopharmaceuticals, Bioanalysis. 9 (2017) 313-326. 


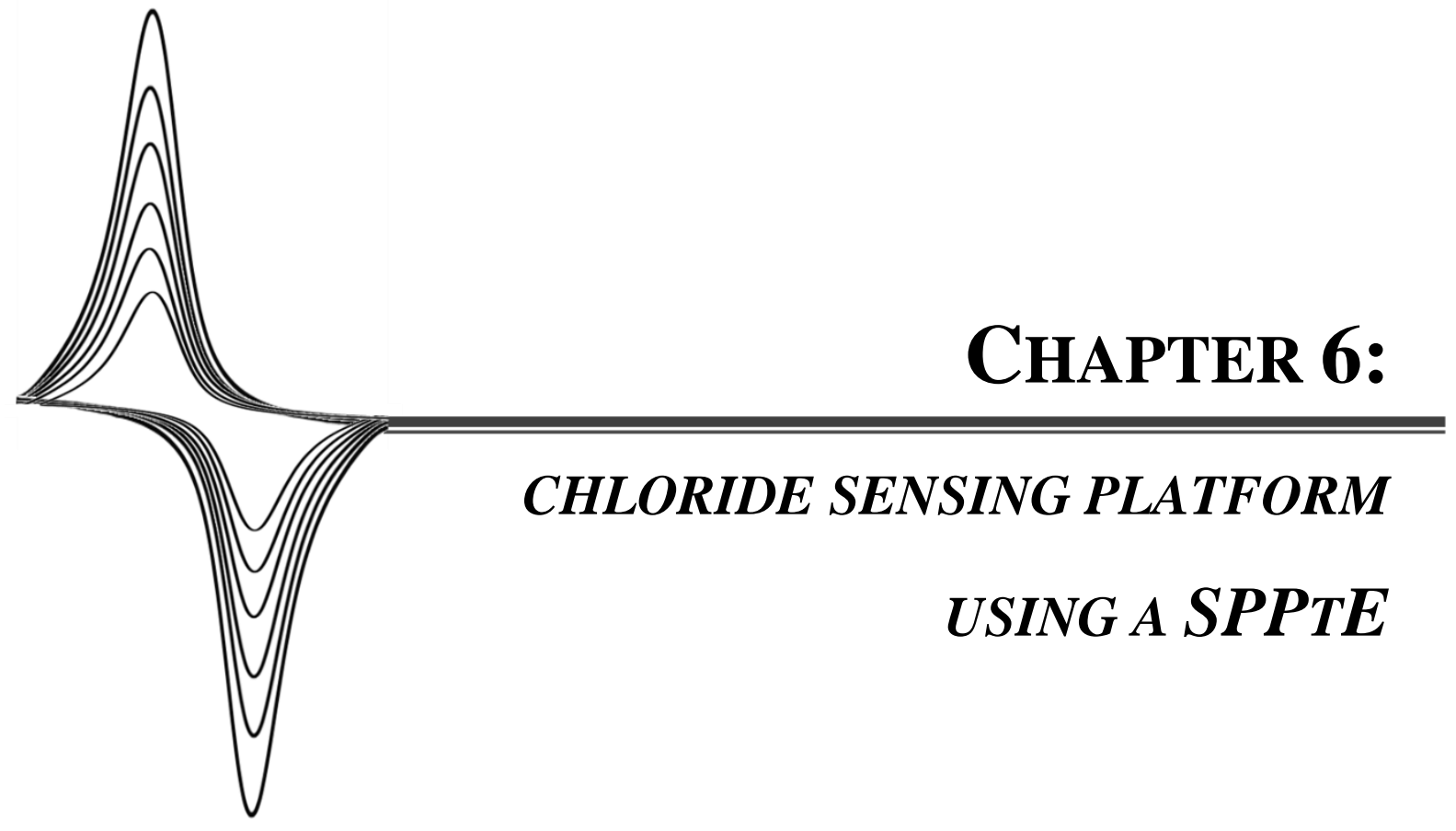



Chloride monitoring is of great importance in health and clinical sectors, where is an indicator for several diseases. Especially important in cystic fibrosis diagnosis, chloride determination is carried out in sweat samples, offering the possibility of noninvasive clinical analysis. Moreover, the concentration of this anion is monitored in pharmaceutical formulations; and also, in food samples where is associated with the salt content of the products.

In the previous chapter 4, it was observed that chloride had an effect on lactate determination, blocking the electrodeposited Pt nanostructures and inhibiting the hydrogen peroxide oxidation onto the modified working electrode. Regarding these results, a bibliographic search shows that several works regarding the fundamentals of the interaction between chloride anions and Pt surfaces, have been already described. However, the use of this adsorptive behavior has not yet been exploited for purposes of chloride anion sensing.

In this chapter is presented a new and selective voltammetric method for chloride determination, based on platinum and chloride interactions. A screen-printed platinum electrode (SPPE) was used as a sensing platform, promoting the formation of chloroadsorbed species on the electrode surface, being an effective means for the anion determination in several matrices. The pretreatment of the SPPtE and careful control of the cathodic stripping voltammetric parameters, yielded a well-defined electrochemical signal. This cathodic peak resulted from the adsorption of chlorine gas, which had previously been oxidized from the sample chloride anions, during the initial anodic deposition step. It offers a simple, low-cost, fast, reproducible $(\mathrm{RSD}<6 \%)$ and precise method for selective chloride determination, with limit of detection of $0.76 \mathrm{mM}$, and a sensitivity of $-24.147 \mu \mathrm{AmM}^{-1}$ for a broad determination range of up to $150 \mathrm{mM}$.

Chloride determination was properly performed with single drops of environmental, pharmaceutical, biological and food samples. In addition, the sensor was successfully adapted as a flexible screen-printed platinum electrode sensor, using Gore-Tex ${ }^{\circledR}$ as support for printing, and applied to sweat samples. 
This work has been patented as "Sensor de detección de ion cloruro en muestras líquidas y procedimiento para la determinación del mismo" (P201830271) and published in Talanta 195, 2019, p. 771-777. 


\subsection{INTRODUCTION}

Chloride ion concentration is an important parameter in various environmental [1-3], pharmaceutical and food-related fields [4,5]. Chloride also has a significant role in biology and medicine, as an essential electrolyte that maintains corporal homeostasis, functioning as a diagnostic indicator of various conditions and diseases. The chloride content of sweat samples is widely analyzed for the diagnosis of cystic fibrosis (CF) [6]. At normal levels, sweat chloride can reach a maximum of $40 \mathrm{mM}$, exceeding 70 or $80 \mathrm{mM}$ in CF positive individuals [6-11]. As the most abundant ion in perspiration, chloride is also a potential biomarker for electrolyte loss in endurance activities [12,13]. A further example is the diagnosis of chloride levels that are outside the normal plasma range of $96-106 \mathrm{mM}$ indicating conditions of hypo or hyperchloremia $[14,15]$. Also present in urine, at concentrations ranging between 110 and $250 \mathrm{mM}$, chloride anion levels can provide information on kidney malfunctions [16,17]. Lower levels of chloride anions are also present in the cerebrospinal fluid of patients with amyotrophic lateral sclerosis [18].

The Mohr volumetric method [19], potentiometric ion selective electrodes (ISE) [20-23], and more recently ion-chromatographic analysis $[24,25]$ are all current standards for chloride determination. Likewise, resonance light scattering [26], turbidimetry [27], colorimetry [28,29], fluorescence [30] and spectrophotometric [31,32] methods, have all been developed. Additionally, some papers published in recent years [33-49] have described several screen-printed electrode (SPE) based electrochemical sensors that perform chloride analysis. The clear advantages of this type of disposable platforms are the low-cost of the materials in use, the possibility of on-site testing, and simple handling of the electrode modifications for their analysis [49]. The majority of studies on chloride determination using SPE are based on the formation of stable compounds between silver and chloride $[33,34,40,47,50]$. The Nernstian shift of a control species voltammetric peak has also been used for chloride determination, since the pseudo-reference $\mathrm{SPE} \mathrm{Ag} / \mathrm{AgCl}$ is 
affected in the presence of this anion [35-38]. Some of these SPE-based sensors printed on flexible substrates are designed to determine sweat chloride. The excellent properties of fabric-based SPEs have also been applied to the development of wearable sensors $[35,50]$.

Even with the interesting results obtained from the above-mentioned methodologies, significant disadvantages still exist. Despite offering continuous measurements, potentiometric methods are time-consuming and require stabilization steps, complicating any routine use. Moreover, ISEs usually have a limited lifetime, requiring regular electrode replacement. Some of the SPE-based sensors that have been described $[35,48]$ need control species, adding possible interferents to the analysis, and requiring sample preparation. Silver-based sensors [33,34,36,40,47,50] present problems such as their complex preparation, electrode fouling, impractical continuous measurement, and reduced concentration ranges.

The demand for an efficient analytical tool calls for the development of new, fast and simple methods of chloride determination. A novel Pt-based SPE sensor is therefore proposed in this paper, in response to the problems found with silver-based SPEs.

Platinum (Pt) is a noble metal, with extensive applications in the electrochemical field. $\mathrm{Pt}$ electrode properties are strongly affected by the presence of chloride ions, particularly when high anodic potentials are applied during the experiments [51-57]. At these potentials, the chloride anions that are specifically adsorbed on the Pt surfaces block the adsorption of oxygen and chlorine gas discharge occurs [57]. Indeed, exhaustive research has already been focused on chloride behavior on Pt surfaces and the fundamentals of its interactions $[51-60]$.

In this study, the feasibility of an electrochemical sensor, using a screen-printed platinum working electrode (SPPtE) is investigated, as an alternative to other approaches for the detection of chloride ions in matrices of a different nature. Although chloride-Pt interactions have been already studied, to the best of our knowledge, only one paper describes those interactions in the context of chlorine gas determination [60]. This method 
achieved a detection limit of only $1.93 \mathrm{mM}$, and a linear range of up to $42 \mathrm{mM}$ that is unsuitable for direct analysis of some chloride samples.

The SPPtE-based sensor developed in this work, uses cathodic stripping voltammetry (CSV) to improve chloride detection. Thus, a simple, fast, low-cost, disposable SPPtEbased sensor was successfully tested for chloride determination in environmental, pharmaceutical and food samples. In addition, a flexible SPPtE sensor was developed that could form the basis for the development of a wearable sensor to monitor the chloride levels of human sweat.

\subsection{EXPERIMENTAL SECTION}

\subsubsection{Chemicals and reagents}

Human plasma, L(-)-malic acid, sodium pyruvate, and D(+)-glucose monohydrate, alanine, aspartic acid, glutamic acid, phenylalanine, glycine, propionic acid, folic acid, iodide, isovaleric acid, leucine, tryptophan, valine, butyric acid, nickel nitrate hexahydrate, calcium sulfate dihydrate, and isobutyric acid, were purchased from Sigma-Aldrich (Steinheim, Germany). L-histidine monohydrochloride monohydrate, sulfuric acid, urea, ascorbic acid, ammonia, sodium phosphate dibasic dihydrate, sodium phosphate monobasic dihydrate, magnesium nitrate, copper(II) sulfate pentahydrate, potassium chloride, and potassium nitrate, were obtained from Merck (Darmstadt, Germany). L-lactic acid (85\%), succinic acid, anhydrous citric acid, ortho-phosphoric acid (85\%), tyrosine, iron ammonium sulfate hexahydrate, cadmium nitrate tetrahydrate, potassium bromide, sodium bicarbonate, potassium sulfate, sodium fluoride, iron ammonium sulfate hexahydrate, and sodium chloride were purchased from Panreac-Applichem (Darmstadt, Germany). Creatinine was obtained from Cromatest (Barcelona), uric acid from Alfa Aesar and acetic acid from VWR (France). 
Commercial wholemeal biscuits, chicken stock, and saline solution were obtained from local markets.

Human sweat and urine samples were collected from healthy volunteers and stored at $-20^{\circ} \mathrm{C}$ before use.

Human plasma was prepared at $0.05 \%(\mathrm{w} / \mathrm{v})$ in a phosphate buffer $(0.31 \mathrm{mM}, \mathrm{pH} 6.2)$ and spiked with two levels of sodium chloride, of $47 \mathrm{mM}$ and $125 \mathrm{mM}$, to simulate conditions of hypo and hyperchloremia, respectively.

Synthetic sweat was formulated, as described elsewhere [13,61], according to the median levels of the compounds found in real human perspiration (Table 6.1).

All reagents were of analytical grade and all solutions were prepared with Milli-Q water (Millipore, Bedford, USA).

The inks used for the fabrication of the SPEs, Electrodag PF-407 A (carbon ink), Electrodag 6037 SS (silver/silver chloride ink), and Electrodag 452 SS (dielectric ink), were supplied by Achenson Colloiden (Scheemda, Netherlands). Polymer platinum ink (C2050804P9) was obtained from Gwent Group (Mamhilad, United Kingdom). Polyester films (PET) of $0.5 \mathrm{~mm}$ thickness (HIFI Industrial Film, Dardily, France) and Gore-Tex ${ }^{\circledR}$ PACLITE ${ }^{\circledR}$ Shell 2-Layer Ripstop Nylon (from Rockywoods Fabrics LLC, Loveland, USA) were used as the printing substrates. 
Table 6.1. Concentration values of human sweat constituents [13,61]. NR - Not reported.

\begin{tabular}{|c|c|c|c|c|}
\hline \multirow{2}{*}{\multicolumn{2}{|c|}{ Constituents }} & \multicolumn{3}{|c|}{ Concentrations (M) } \\
\hline & & Median & Minimum & Maximum \\
\hline \multirow{15}{*}{ 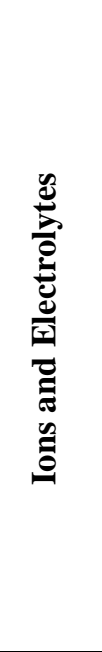 } & Phosphate & $3.1 \times 10^{-4}$ & $2.3 \times 10^{-5}$ & $1.1 \times 10^{-3}$ \\
\hline & Bicarbonate & $3.0 \times 10^{-3}$ & $2.6 \times 10^{-4}$ & $2.0 \times 10^{-2}$ \\
\hline & Sulfur & $2.3 \times 10^{-3}$ & $2.2 \times 10^{-4}$ & $2.3 \times 10^{-3}$ \\
\hline & Sulfate & $4.2 \times 10^{-4}$ & $7.0 \times 10^{-5}$ & $2.0 \times 10^{-3}$ \\
\hline & Iodide & $7.1 \times 10^{-8}$ & $7.9 \times 10^{-11}$ & $7.5 \times 10^{-5}$ \\
\hline & Bromide & $2.3 \times 10^{-6}$ & $4.4 \times 10^{-9}$ & $6.3 \times 10^{-6}$ \\
\hline & Fluoride & $1.1 \times 10^{-6}$ & $5.8 \times 10^{-8}$ & $9.5 \times 10^{-5}$ \\
\hline & Chloride & $2.3 \times 10^{-2}$ & $1.7 \times 10^{-5}$ & $2.8 \times 10^{-1}$ \\
\hline & Calcium & $5.2 \times 10^{-3}$ & $4.7 \times 10^{-6}$ & $1.5 \times 10^{-2}$ \\
\hline & Potassium & $6.1 \times 10^{-3}$ & $6.7 \times 10^{-6}$ & $3.8 \times 10^{-2}$ \\
\hline & Magnesium & $8.2 \times 10^{-5}$ & $7.4 \times 10^{-8}$ & $3.8 \times 10^{-3}$ \\
\hline & Cadmium & $1.8 \times 10^{-8}$ & $1.2 \times 10^{-8}$ & $2.3 \times 10^{-8}$ \\
\hline & Copper & $9.4 \times 10^{-7}$ & $1.3 \times 10^{-8}$ & $1.2 \times 10^{-3}$ \\
\hline & Iron & $9.8 \times 10^{-6}$ & $1.5 \times 10^{-8}$ & $1.1 \times 10^{-3}$ \\
\hline & Niquel & $4.2 \times 10^{-7}$ & $1.7 \times 10^{-9}$ & $8.3 \times 10^{-7}$ \\
\hline \multirow{8}{*}{ 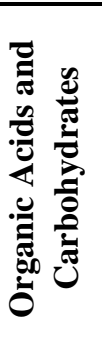 } & Lactic acid & $1.4 \times 10^{-3}$ & $3.4 \times 10^{-3}$ & $5.0 \times 10^{-2}$ \\
\hline & Pyruvic acid & $1.8 \times 10^{-4}$ & $1.0 \times 10^{-7}$ & $1.0 \times 10^{-3}$ \\
\hline & Butyric acid & $2.4 \times 10^{-6}$ & $5.0 \times 10^{-7}$ & $6.0 \times 10^{-6}$ \\
\hline & Acetic acid & $1.3 \times 10^{-4}$ & $5.9 \times 10^{-5}$ & $4.2 \times 10^{-4}$ \\
\hline & Propionic acid & $3.5 \times 10^{-6}$ & $1.2 \times 10^{-6}$ & $7.4 \times 10^{-6}$ \\
\hline & Isobutyric acid & $8.0 \times 10^{-7}$ & NR & $2.8 \times 10^{-6}$ \\
\hline & Isovaleric acid & $1.1 \times 10^{-6}$ & $2.0 \times 10^{-7}$ & $4.5 \times 10^{-6}$ \\
\hline & Glucose & $1.7 \times 10^{-4}$ & $5.6 \times 10^{-6}$ & $2.2 \times 10^{-3}$ \\
\hline \multirow{10}{*}{ 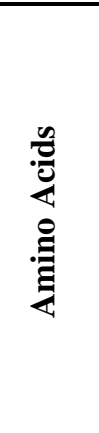 } & Alanine & $3.6 \times 10^{-4}$ & NR & NR \\
\hline & Aspartic acid & $3.4 \times 10^{-4}$ & NR & NR \\
\hline & Glycine & $3.9 \times 10^{-4}$ & NR & NR \\
\hline & Glutamic acid & $3.7 \times 10^{-4}$ & NR & NR \\
\hline & Histidine & $5.2 \times 10^{-4}$ & $2.7 \times 10^{-4}$ & $1.3 \times 10^{-2}$ \\
\hline & Leucine & $2.7 \times 10^{-4}$ & $9.1 \times 10^{-5}$ & $3.2 \times 10^{-4}$ \\
\hline & Phenylalanine & $1.3 \times 10^{-4}$ & $6.1 \times 10^{-5}$ & $2.1 \times 10^{-4}$ \\
\hline & Tryptophan & $5.5 \times 10^{-5}$ & $2.0 \times 10^{-5}$ & $9.1 \times 10^{-5}$ \\
\hline & Tyrosine & $1.7 \times 10^{-4}$ & $6.6 \times 10^{-5}$ & $3.0 \times 10^{-4}$ \\
\hline & Valine & $2.5 \times 10^{-4}$ & $1.3 \times 10^{-4}$ & $3.8 \times 10^{-4}$ \\
\hline \multirow{6}{*}{ 离 } & Ammonia & $5.2 \times 10^{-3}$ & $4.7 \times 10^{-4}$ & $2.5 \times 10^{-2}$ \\
\hline & Uric acid & $5.9 \times 10^{-5}$ & $4.2 \times 10^{-6}$ & $4.8 \times 10^{-3}$ \\
\hline & Urea & $10 \times 10^{-2}$ & $1.8 \times 10^{-3}$ & $4.6 \times 10^{-2}$ \\
\hline & Creatinine & $8.4 \times 10^{-5}$ & $8.8 \times 10^{-6}$ & $2.0 \times 10^{-3}$ \\
\hline & Folic acid & $1.0 \times 10^{-5}$ & $1.1 \times 10^{-7}$ & $3.6 \times 10^{-5}$ \\
\hline & Ascorbic acid & $1.0 \times 10^{-5}$ & $1.1 \times 10^{-7}$ & $3.3 \times 10^{-5}$ \\
\hline
\end{tabular}




\subsubsection{Apparatus}

Voltammetric measurements were performed using a PalmSens ${ }^{\circledR}$ portable electrochemical potentiostat with the PS Trace 4.2 program (PalmSens ${ }^{\circledR}$ Instruments BV, Houten, The Netherlands).

The potentiometric analysis was performed with a $\mathrm{pH} \&$ Ion-Meter GLP 22+, connected to a chloride ion selective electrode 9652 C, from CRISON Instruments, S.A. (Barcelona, Spain).

The conventional three-electrode system was obtained from BASI bioanalytical systems (West Lafayette, USA). It consisted of three electrode cells, namely a platinum electrode with a diameter of $1.6 \mathrm{~mm}$ (MF-2013), a platinum wire electrode (CHI115), and a silver/silver chloride electrode (MF-2025), used as the working, counter, and reference electrodes, respectively.

Microscopy imaging and elemental composition analysis were performed using a scanning electron microscope (SEM) JEOL JSM-6460LV with an INCA elementary X-ray analysis system Oxford Instruments (Abingdon-on-Thames, UK). The X-ray analysis was performed at three different zones of each working electrode with a zoom of x500.

\subsubsection{SPE manufacturing and preparation}

The SPE systems were fabricated by sequential ink layer deposition on PET and Gore-Tex ${ }^{\circledR}$ supports, previously heated and held at a temperature of $120^{\circ}$ for $2 \mathrm{~h}$. SPE manufacturing was completed following previously described procedures [62]. Briefly, silver and silver/silver chloride inks, cured at $120{ }^{\circ} \mathrm{C}$ for $20 \mathrm{~min}$, were used to print the conductive tracks and the reference electrodes, respectively. The counter electrode was then printed with carbon ink and cured at $60{ }^{\circ} \mathrm{C}$ for $30 \mathrm{~min}$. Next, platinum ink was screen-printed and cured at $80{ }^{\circ} \mathrm{C}$ for $30 \mathrm{~min}$. to define the Pt working electrodes (SPPtE) $\left(\operatorname{area}=12.56 \mathrm{~mm}^{2}\right)$. Finally, dielectric ink, which defines the final geometry of the three electrodes, was printed and cured under the same conditions. 
Before the chloride analysis, each electrode was electrochemically polished in $\mathrm{KNO}_{3} 0.1$ $\mathrm{M}$, by applying 5 cyclic voltammetric scans from -0.20 to $-1.50 \mathrm{~V}$ at $0.05 \mathrm{~V} \mathrm{~s}^{-1}$ (Figure 6.3. A).

\subsubsection{Cyclic voltammetric measurements}

The screening of electrochemical behavior of chloride at SPPtE, was carried out using cyclic voltammetric (CV) experiments, performed by drop casting $200 \mu \mathrm{L}$ of supporting electrolyte (phosphate buffer, $0.31 \mathrm{mM}, \mathrm{pH}$ 6.2), containing 5, 10, 20, 30, 60, 80, and 100 $\mathrm{mM} \mathrm{NaCl}$, onto the electrode surface. Voltammograms were recorded, between $-0.70 \mathrm{~V}$ and $+1.30 \mathrm{~V}(\mathrm{vs} \mathrm{SPE} \mathrm{Ag} / \mathrm{AgCl})$ at $0.1 \mathrm{~V} \mathrm{~s}^{-1}$.

Randles-Sevcik CV experiments, were performed by drop-casting $200 \mu \mathrm{L}$ of supporting electrolyte containing $60 \mathrm{mM} \mathrm{NaCl}$, onto the electrodic system, from +0.40 to $+1.30 \mathrm{~V}$ (vs SPE $\mathrm{Ag} / \mathrm{AgCl}$ ), at the following scan-rate values: 0.025, 0.050, 0.075, 0.100, 0.150 and $0.200 \mathrm{~V} \mathrm{~s}^{-1}$.

The experiments using the conventional three-electrode cell system, were performed from +0.40 to $+1.30 \mathrm{~V}$ (vs $\mathrm{Ag} / \mathrm{AgCl}$ ) in a cell containing $10 \mathrm{~mL}$ of supporting electrolyte, and the desired chloride concentration, spiked from a stock solution of $\mathrm{NaCl} 3 \mathrm{M}$, at $0.1 \mathrm{~V} \mathrm{~s}^{-1}$.

\subsubsection{Cathodic stripping voltammetry for chloride determination}

The cathodic stripping voltammetric (CSV) experiments were performed by drop casting $200 \mu \mathrm{L}$ of supporting electrolyte containing different concentrations of $\mathrm{NaCl}$ onto the electrodic surface. A first deposition step at $+1.50 \mathrm{~V}$ was applied over 15 seconds, followed immediately (equilibration time $0 \mathrm{~s}$ ) by a cathodic linear sweep voltammetric scan, from +1.11 to $+0.10 \mathrm{~V}$ at a scan rate of $0.20 \mathrm{~V} \mathrm{~s}^{-1}$, returning a reduction peak at $+0.80 \mathrm{~V}$ in electropolished electrode. 


\subsubsection{Chloride ion selective electrode measurement}

A chloride ion selective electrode ( $\mathrm{Cl}$ - ISE) was the reference method used to corroborate the voltammetric results. The experiments were performed in stabilization mode and the potential value recorded after measurement stabilization. According to the supplier, chloride standards from 0.5 to $60 \mathrm{mM}$ of $\mathrm{KCl}$ were prepared in $\mathrm{KNO}_{3} 0.01 \mathrm{M}$. Calibration curve $(\log [\mathrm{KCl}](\mathrm{mM})$ vs potential $(\mathrm{mV}))$, was used to determine the chloride content in samples.

\subsection{RESULTS AND DISCUSSION}

\subsubsection{Chloride voltammetric peak screening}

As previously mentioned, several authors have described specific interactions between chloride and platinum surfaces, once this anion leads to the inhibition of redox processes [52], competing with oxides and protons that are usually adsorbed [55].

Confirmation of those sorts of interactions at the SPPtE was carried out by screening the voltammetric peak in the presence of chloride through CV scans. In Figure 6.1, numerous peaks appear within the CV experimental data. Special attention was given to the cathodic peak developed at $+1.0 \mathrm{~V}$, as its potential remained stable, showing a relation with chloride concentrations. This agrees with the findings of Kuhn and Wright for platinum electrode behavior in strong chloride solutions [51]. This peak may be related with the chlorine gas generated on the working electrode surface. No stable oxidation signal was observed in the $\mathrm{CV}$ scans, to pair it with the reduction signal, so several experiments, were performed to determine the cause of the reduction. The results obtained for potential sweeps, by means of CSV performed from +1.3 to $0 \mathrm{~V}$, were therefore analyzed. 


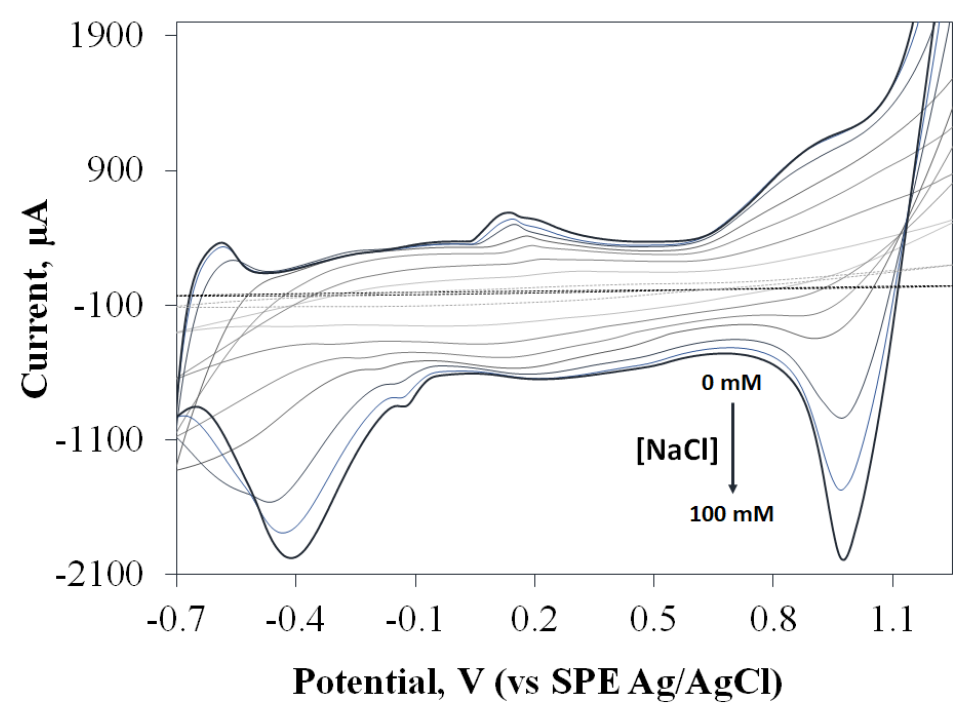

Figure 6.1. $C V$ scans from -0.7 to $+1.3 \mathrm{~V}$ recorded at SPPtE in a $200 \mu \mathrm{L}$ drop of supporting electrolyte, containing 0, 1, 5, 10, 20,30, 60, 80 and $100 \mathrm{mM}$ of $\mathrm{NaCl}$. Scan rate $0.1 \mathrm{~V} \mathrm{~s}^{-1}$.

\subsubsection{CSV setting for chloride quantification}

CSV was found to be the ideal technique to follow the reduction peak developed in the presence of chloride ions. Previous experiments have shown that the current signal is notably increased when an anodic pretreatment step is performed before the cathodic potential sweep. In doing so, the influence of both anodic potential $\left(E_{d e p}\right)$ and time deposition $\left(t_{d e p}\right)$ pretreatment can be considered. In this study, an $E_{d e p}$ of $+1.5 \mathrm{~V}$ was applied over $15 \mathrm{~s}$ as the optimal CSV conditions (Figure 6.2).

\subsubsection{SPPtE electropolishing}

When assessing the performance of the sensor for chloride determination, under the previously adjusted conditions, it was found that the voltammetric reduction peaks were irreproducible and unstable. An electropolishing step of the SPPtE in $0.1 \mathrm{M} \mathrm{KNO}_{3}$ was therefore performed (Section 6.2.3. SPE Manufacturing and Preparation). The cyclic voltammograms obtained for electropolishing step, exhibits a different behavior in the first 
scan, since the reduction peak developed at $-1.0 \mathrm{~V}$ disappeared over repeated $\mathrm{KNO}_{3}$ scans (Figure 6.3. A).
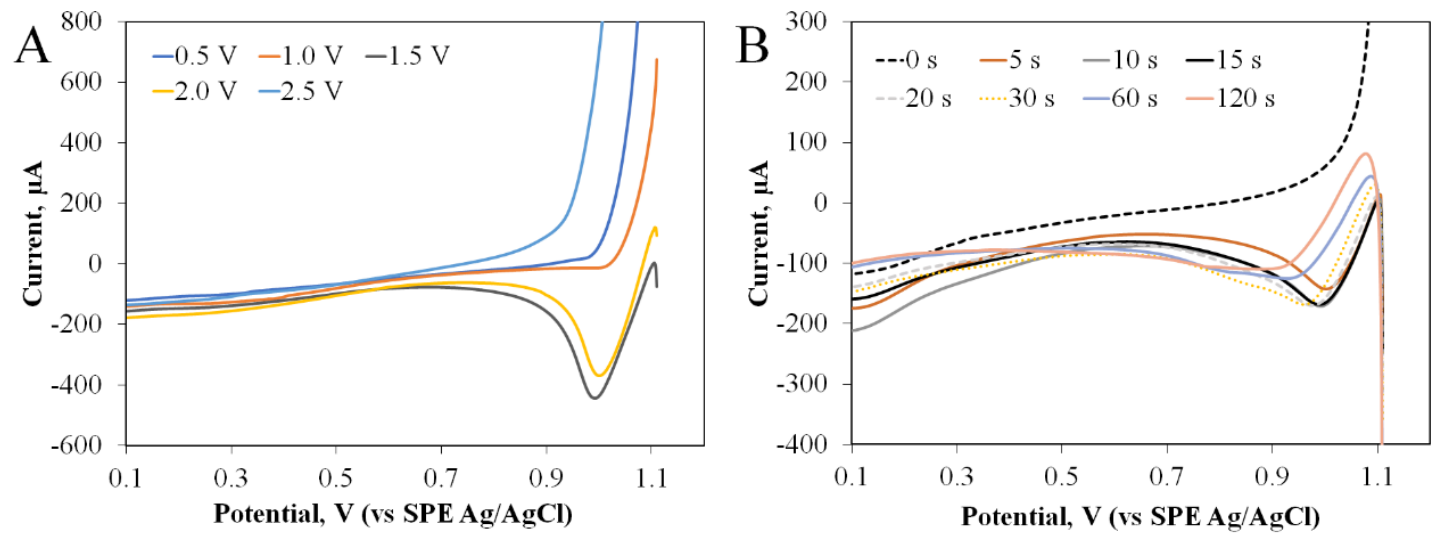

Figure 6.2. Anodic step optimization. CSV recorded from +1.11 to $+0.10 \mathrm{~V}$ at SPPtE for $200 \mu \mathrm{L}$ of $30 \mathrm{mM}$ of $\mathrm{NaCl}$ for: (A) $E_{\text {dep }}$ of 0.5, 1.0, 1.5, 2.0 and $2.5 \mathrm{~V}$, during $t_{\text {dep }} 5 \mathrm{~s}$; and (B) $E_{\text {dep }}$ of +2.0 $V$, during $t_{\text {dep. }} .0,5,10,15,20,30,60$ and $120 \mathrm{~s}$. Scan rate: $0.02 \mathrm{~V} \mathrm{~s}^{-1}$.
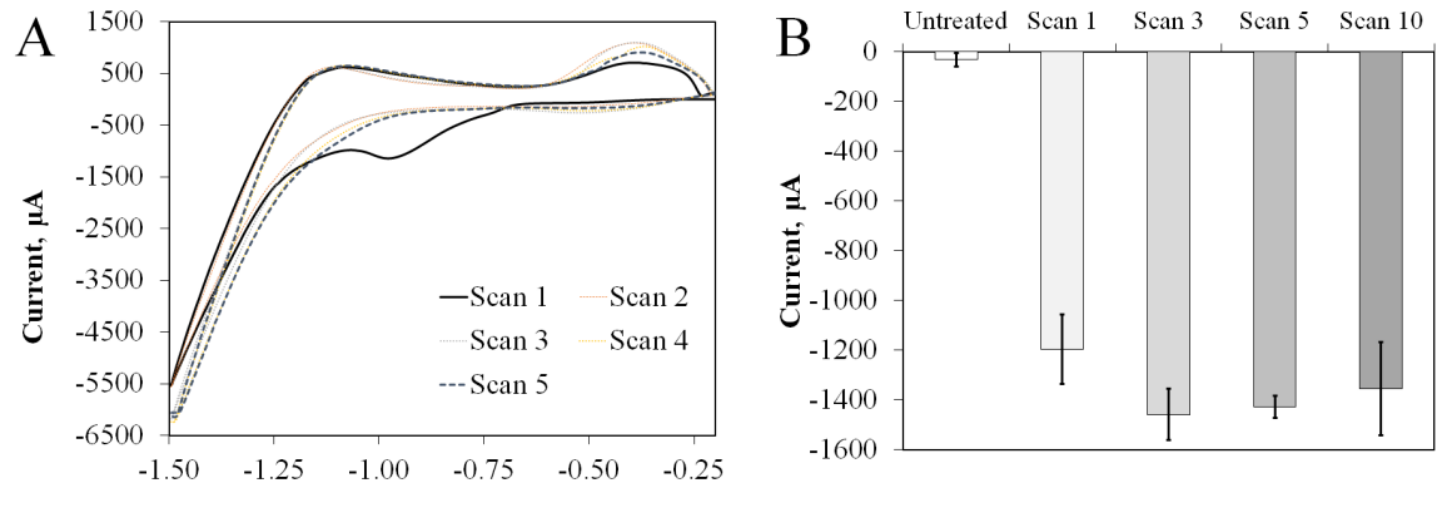

Potential, V (vs SPE Ag/AgCl)

Figure 6.3. SPPtE electropolishing treatment. (A) CV scans at SPPtE of electrochemical polishing. Five scans performed in $0.1 \mathrm{M} \mathrm{KNO}_{3}$, from -0.25 to $-1.5 \mathrm{~V}$. Scan rate $0.1 \mathrm{~V} \mathrm{~s}^{-1}$; and (B) CSV peak current for NaCl $30 \mathrm{mM}$, after 1, 3, 5 and 10 scans at EP/SPPtE. Error bars represent the standard deviation of three independent SPPtEs. 
With the purpose of detecting modifications in the typical proton and oxygen adsorption zones on Pt surfaces, the effect of this step was studied in acidic media [63]. Thus, when $0.1 \mathrm{M} \mathrm{H}_{2} \mathrm{SO}_{4}$ was used as a supporting electrolyte, the untreated SPPtE exhibited a flat CV profile, with low current densities and imperceptible signals. Contrasting data were found when using an electropolished working electrode (SPPtE/EP), which recorded increased current outputs and a well-marked CV profile, displaying the expected peaks shapes and positions usually found for a Pt surface (Figure 6.4). This treatment generates a higher number of active sites for the reaction on the electrode surface. These data might be related with the presence of a polycrystalline nanosurface of platinum available to interact with reactants $[51,55,63]$, as a result of the elimination of Pt ink impurities.

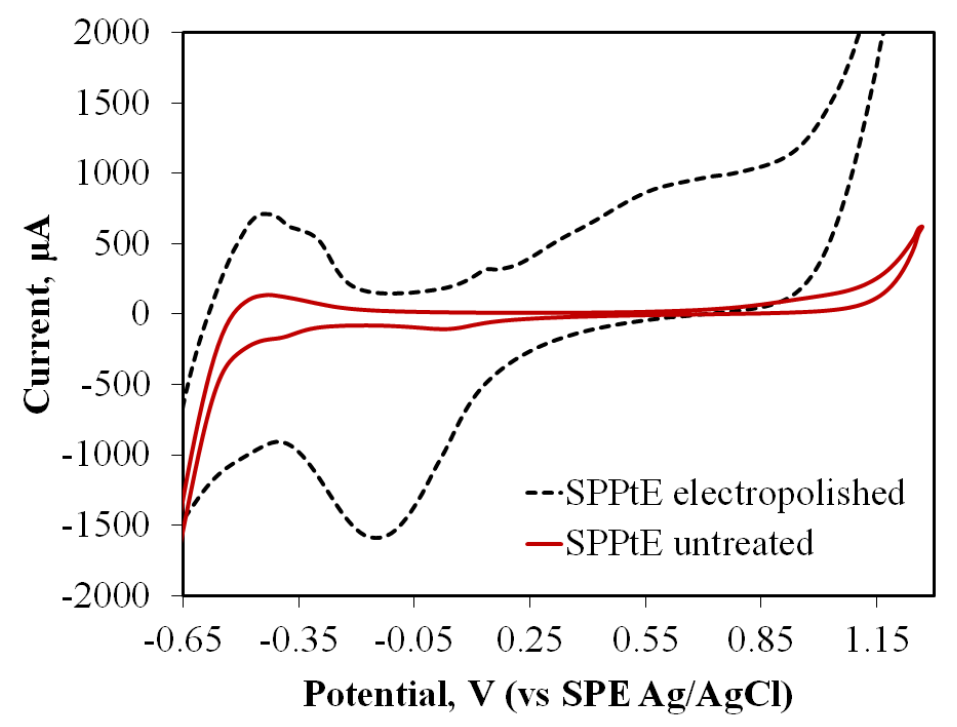

Figure 6.4. $\mathrm{CV}$ scans from -0.65 to $+1.30 \mathrm{~V}$ recorded at SPPtE in a $200 \mu \mathrm{L}$ drop of $\mathrm{H}_{2} \mathrm{SO}_{4}(0.1 \mathrm{M})$. Scan rate $0.05 \mathrm{~V} \mathrm{~s}^{-1}$.

The data obtained in the X-ray elemental composition analysis of the SPPtE/EP surface, confirmed the increase of a more electroactive surface, revealing a $15 \%$ increment in oxygen composition, when compared with an untreated SPPtE (Table 6.2). The chloride 
reduction peak was also shifted from +1.0 to $+0.8 \mathrm{~V}$ (vs $\mathrm{SPE} \mathrm{Ag} / \mathrm{AgCl}$ ) when the CSV experiments were performed, which confirmed that the chloride redox process occurred more easily and extensively on a treated surface, rather than on an untreated SPPtE.

Table 6.2. X-Ray elemental analysis of SPPtE surface at different steps of voltammetric analysis.

\begin{tabular}{lcccc}
\hline \multicolumn{1}{c}{ Step of analysis } & \multicolumn{1}{c}{$\boldsymbol{w t}(\boldsymbol{\%})$} & $\boldsymbol{c}$ \\
\hline $\mathrm{SPPtE}$ & $89.26 \pm 0.15$ & $2.73 \pm 0.10$ & $0.00 \pm 0.00$ \\
$\mathrm{SPPtE} / \mathrm{EP}$ & $85.94 \pm 3.26$ & $3.29 \pm 0.25$ & $0.00 \pm 0.00$ \\
$\mathrm{SPPtE} / \mathrm{EP} / \mathrm{E}_{\text {dep }} 0 \mathrm{mM} \mathrm{NaCl}$ & $88.33 \pm 0.57$ & $3.41 \pm 0.55$ & $0.00 \pm 0.00$ \\
$\mathrm{SPPtE} / \mathrm{EP} / \mathrm{E}_{\text {dep }} 10 \mathrm{mM} \mathrm{NaCl}$ & $88.46 \pm 0.98$ & $2.96 \pm 0.50$ & $0.50 \pm 0.05$ \\
$\mathrm{SPPtE} / \mathrm{EP} / \mathrm{E}_{\text {dep }} 150 \mathrm{mM} \mathrm{NaCl}$ & $87.67 \pm 0.38$ & $2.51 \pm 0.15$ & $0.80 \pm 0.05$ \\
$\mathrm{SPPtE} / \mathrm{EP} / \mathrm{E}_{\text {dep }} 10 \mathrm{mM} \mathrm{NaCl} / \mathrm{CSV}$ & $90.64 \pm 0.26$ & 2.31 & \pm 0.20 & $0.00 \pm 0.00$ \\
$\mathrm{SPPtE} / \mathrm{EP} / \mathrm{E}_{\text {dep }} 150 \mathrm{mM} \mathrm{NaCl} / \mathrm{CSV}$ & $88.98 \pm 0.20$ & $3.02 \pm 0.26$ & $0.00 \pm 0.00$ \\
\hline
\end{tabular}

SPPtE - untreated electrode; SPPtE/EP - After electropolishing step $\left(0.1 \mathrm{M}\right.$ of $\left.\mathrm{KNO}_{3}\right)$; SPPtE/EP/E $\mathrm{E}_{\mathrm{dep}}-\mathrm{After}$ the anodic deposition $(1.5 \mathrm{~V}, 15 \mathrm{~s})$ for 0,10 , and $150 \mathrm{mM}$ of NaCl; SPPtE/EP/E $\mathrm{E}_{\text {dep }} / \mathrm{CSV}$ - After CSV (from 1.10 to $0.1 \mathrm{~V}$ at 0,10 and $150 \mathrm{mM}$ of $\mathrm{NaCl}$ ).

The chloride voltammetric peak current was remarkably increased at SPPtE/EP and the number of scans improved the anion determination. Five cycles were selected for the electropolishing step, as lower deviations were found between replicas (Figure $6.3 \mathrm{~B}$ ).

An additional study, using a conventional (not screen-printed) three-electrode cell system with a Pt working electrode, was performed in the presence of chloride, before and after $\mathrm{KNO}_{3}$ electropolishing. As observed using a SPPtE, under the $\mathrm{CV}$ conditions employed for chloride voltammetric peak screening $(\mathrm{CV}$ from -0.7 to $+1.3 \mathrm{~V}$, at different chloride concentrations, in phosphate buffer, $0.31 \mathrm{mM}, \mathrm{pH}$ 6.2), the conventional Pt electrode developed a well-defined reduction peak at $+1.0 \mathrm{~V}(\mathrm{vs} \mathrm{Ag} / \mathrm{AgCl})$, which current is related 
with chloride concentrations (Figure 6.5). Nevertheless, the electropolishing of this conventional Pt electrode, displayed a different behavior to those observed at the SPPtE, developing a flat behavior over the five scans (Figure 6.6). This Pt surface also behaved differently in CSV experiments, originating weak reduction peaks that can barely be related with chloride concentrations. Even though poor linearity was found for the chloride calibration at this conventional Pt surface, $\mathrm{KNO}_{3}$ electropolishing also increased the current output for the cathodic peaks (Figure 6.7).

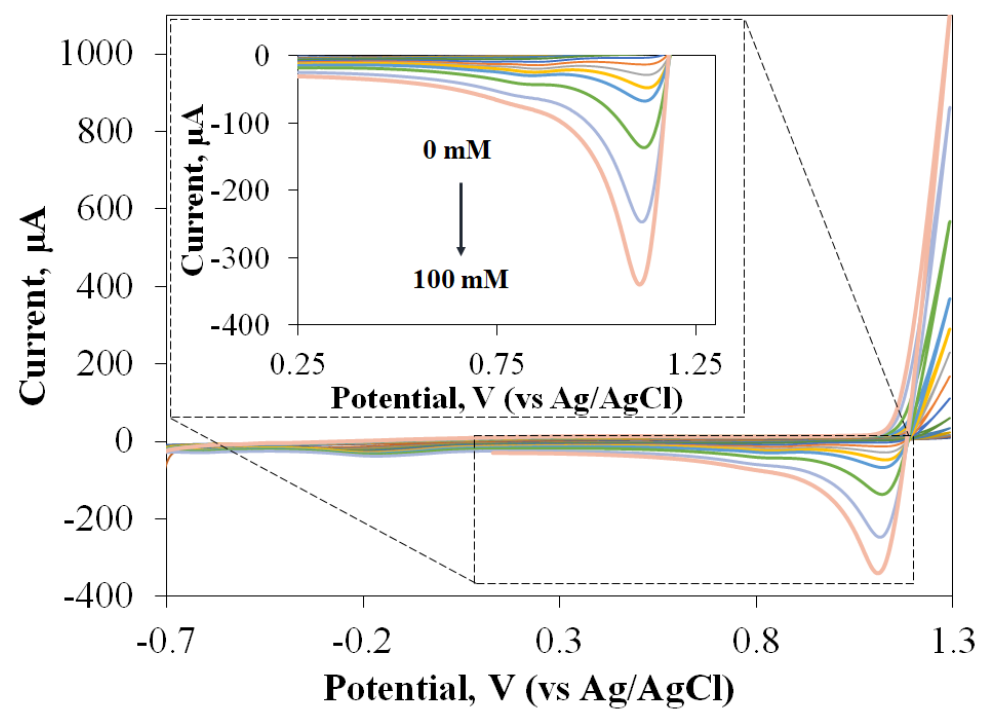

Figure 6.5. $C V$ scans from -0.7 to $+1.3 \mathrm{~V}$ in supporting electrolyte, containing $0,1,5,10,20,30$, 60, 80 and $100 \mathrm{mM}$ of $\mathrm{NaCl}$, performed in conventional Pt electrode.

As previously mentioned, the $\mathrm{Pt}$ ink is also crucial for chloride determination, as the conventional metallic Pt electrode proved itself ineffective at obtaining a satisfactory response in the presence of the anion. Therefore, all the experiments conducted for the characterization, application and validation of the proposed sensor, were performed with previously electropolished SPPtEs. This step, performed just before the first utilization, demonstrated stable measurements. 


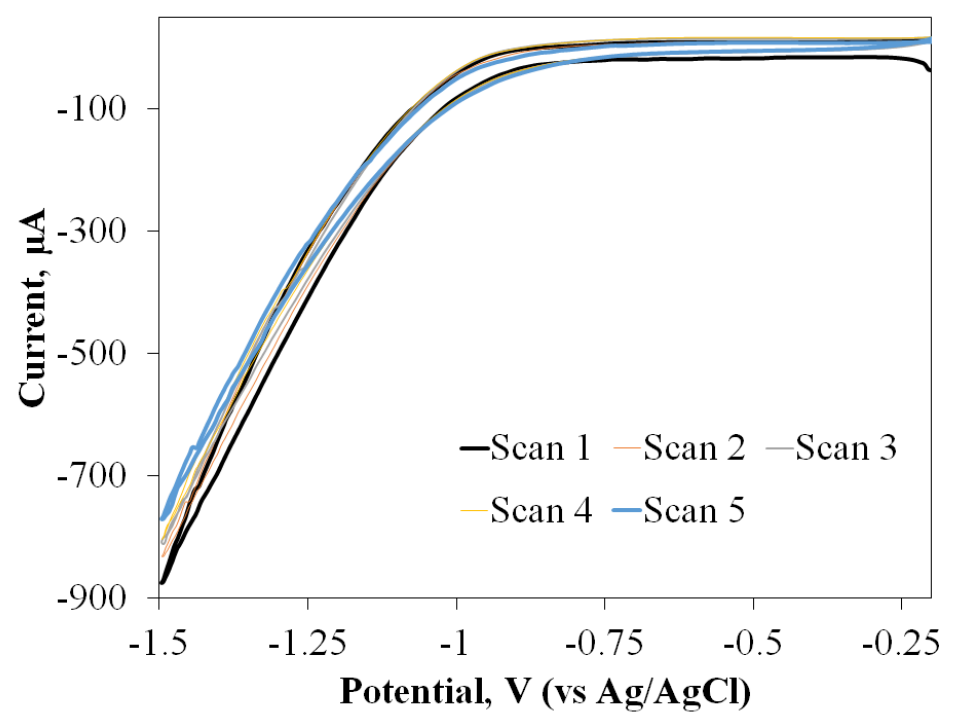

Figure 6.6. $C V$ scans at conventional Pt electrode of electrochemical polishing. Five scans performed in $0.1 \mathrm{M} \mathrm{KNO}_{3}$, from -0.20 to $-1.5 \mathrm{~V}$. Scan rate $0.1 \mathrm{~V} \mathrm{~s}^{-1}$.
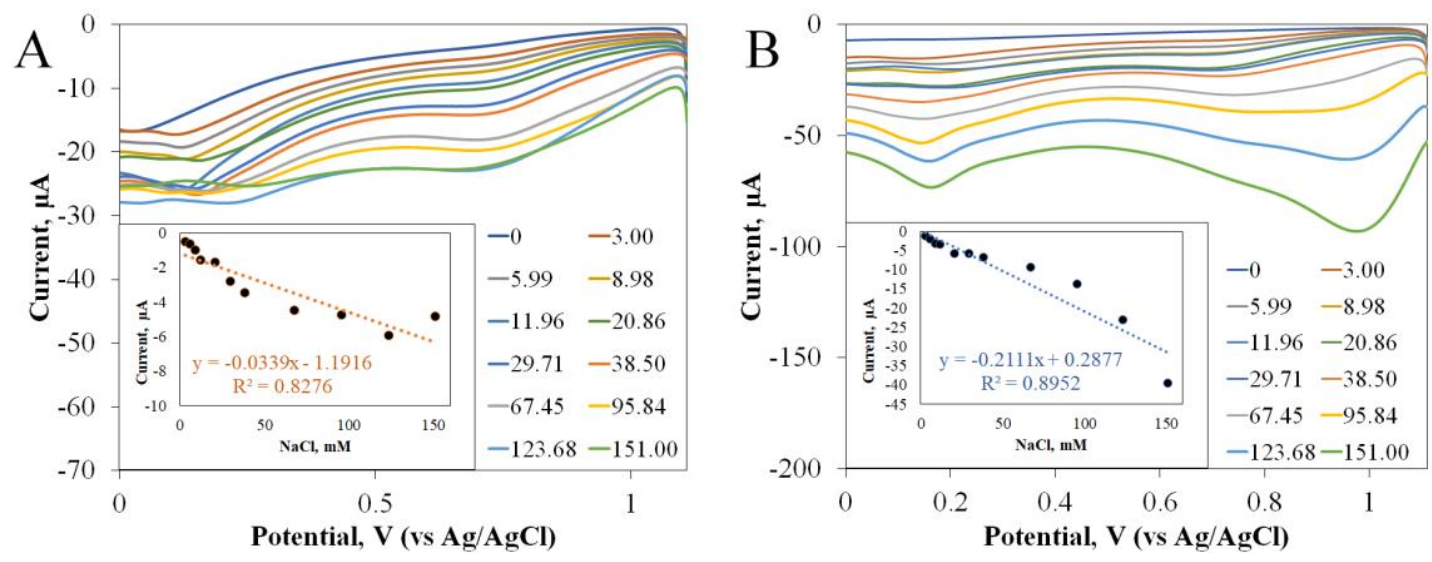

Figure 6.7. CSV scans after anodic deposition step at: (A) conventional no treated Pt electrode, and (B) $0.1 \mathrm{M} \mathrm{KNO}_{3}$ electropolished Pt electrode. Insets: Respective calibration plots. $\left(E_{\text {dep }}:+1.5\right.$ $V$, $t_{\text {dep }} 15 \mathrm{~s}$, scan rate of $\left.0.02 \mathrm{~V} \mathrm{~s}^{-1}\right)$. 


\subsubsection{Electrochemical mechanism for CSV chloride reduction peak}

It has been demonstrated that the reduction peaks observed in CSV experiments can be related with chlorine evolution; however, it involves a complex mechanism. In fact, many processes may occur on the electrode surface involving both faradaic and nonfaradaic contributions. A chemisorption electrochemical mechanism is proposed for a better understanding of the voltammetric reduction peak and its generation in chloride containingmedia. The mechanism may rely on the formation of chloro-complexes on the Pt surface, which are oxidized during the anodic pretreatment, applied just before the cathodic sweep. Somewhat conflicting information exists regarding the type of complexes formed; nevertheless, there is agreement that an oxide layer deposited on the Pt surface will favor chloride complexation with the metal. Some of those complexes are described as $\mathrm{PtCl}^{-}$, $\mathrm{PtCl}_{2}, \mathrm{PtCl}_{4}{ }^{2-}$ and $\mathrm{PtCl}_{6}{ }^{2-}$ [51-57]. Other studies, in agreement with the results found using the proposed system, have indicated the presence of strongly adsorbed chlorine atoms in the electrode reaction mechanism [58-60].

X-ray analyses of the different steps of sensor operation for chloride analysis was used to evaluate changes in the SPPtEs surface composition. The wt \% of each element was compared on various surfaces: untreated SPPtE (identified as SPPtE in table 6.2), after the $\mathrm{KNO}_{3}$ electropolishing treatment (identified as SPPtE/EP in table 6.2), when submitted to an anodic treatment at a $E_{d e p}$ of $1.5 \mathrm{~V}$ applied during $15 \mathrm{~s}$ under different chloride concentrations in the solution (identified as SPPtE/EP/E $E_{d e p} 0,10$ and $150 \mathrm{mM} \mathrm{NaCl}$ in table 6.2), and after the cathodic sweep, also at different chloride concentrations (identified as SPPtE/EP/E $E_{\text {dep }} 0,10$ and $150 \mathrm{mM} \mathrm{NaCl/CSV}$ in table 6.2). The untreated SPPtE presented a small percentage of oxygen, limiting the formation of chloro-adsorbed complexes that originated small voltammetric reduction peaks. The oxide layer was increased after the electropolishing step (SPPtE/EP), which prompted extensive interaction between chloride and Pt, resulting in improved sensitivity to the anion. This is also noticeable for the Pt oxide peak current, which is improved at a SPPtE/EP (Figure 6.4).

Moreover, when the anodic deposition step was performed without the cathodic potential sweeping $\left(S P P t E / E P / E_{d e p} \mathrm{NaCl}\right)$, a wt $\%$ of chloride was found on the electrode surface. 
However, when chloride was absent in the anodic deposition step, no compositional change was perceptible in comparison with the SPPtE/EP surface. In this step, surface chloride and oxygen also appeared to be related with the lowest wt \% of oxygen that was present when the chloride concentration was increased. This observation lends support to the idea that the formation of chloro-adsorbed complexes requires a previously formed oxide layer, which promoted interaction between anions and the Pt surface. The X-ray elemental analysis, summarized in table 6.2, also offers information on the electrochemical reduction of adsorbed chlorine gas, confirming the disappearance of chloride from the Pt surface, after the cathodic potential sweep (SPPtE/EP/E $E_{d e p} 10$ and $\left.150 \mathrm{mM} \mathrm{NaCl/CSV}\right)$.

The X-ray data showed that the mechanism relies on chloride complexation at the electropolished Pt surface, where its oxidization releases chlorine gas during the anodic step (equation 6.1). During this step, gas bubble emerges from the sample, revealing that chlorine generation was occurring at these high potentials. Subsequently, the electrochemically generated chlorine gas was reduced to chloride anions during the cathodic potential sweeping (equation 6.2); regenerating the oxide layer at less positive potentials and recycling the Pt surface for a new reaction. It is suggested that this behavior results from a nondissociative adsorption of chlorine gas on the Pt electrode surface, which is followed by electron transfer [60].

$$
\begin{aligned}
& 2 \mathrm{Cl}^{-} \rightarrow \mathrm{Cl}_{2 \text { ads }}+2 e^{-} \\
& C l_{2 \text { ads }}+2 e^{-} \rightarrow 2 C l^{-}
\end{aligned}
$$

Murugappan and co-workers proposed a similar mechanism for the detection of chloride gas on a Pt microdisk. Their conclusion was that chlorine gas must partition into the liquid phase before chlorine reduction occurs at the electrode surface [60]. In our system, the chlorine gas is formed on the working electrode and partitioning may also occur. Even so, nondissociative chlorine gas absorption may be favored, as the chlorine is generated in situ and is then reduced to its corresponding anions. 
The figure 6.8 shows SEM SPPtE imaging of a rough surface, constituted by spherical microstructures of platinum that are homogeneous throughout the different steps of the analysis. This rough surface also contributes to the nondissociative chlorine gas absorption mechanism, playing an important role in sensor performance. So, it can be assumed that the successive steps converged in the improved effectiveness of the SPPtE to react, rather than alter its surface morphology. Furthermore, the Randles-Sevcik equation, produced a non-linear relation; indicating that this mechanism is not controlled by diffusion, with the possible involvement of adsorbed intermediates (Figure 6.9) [64].
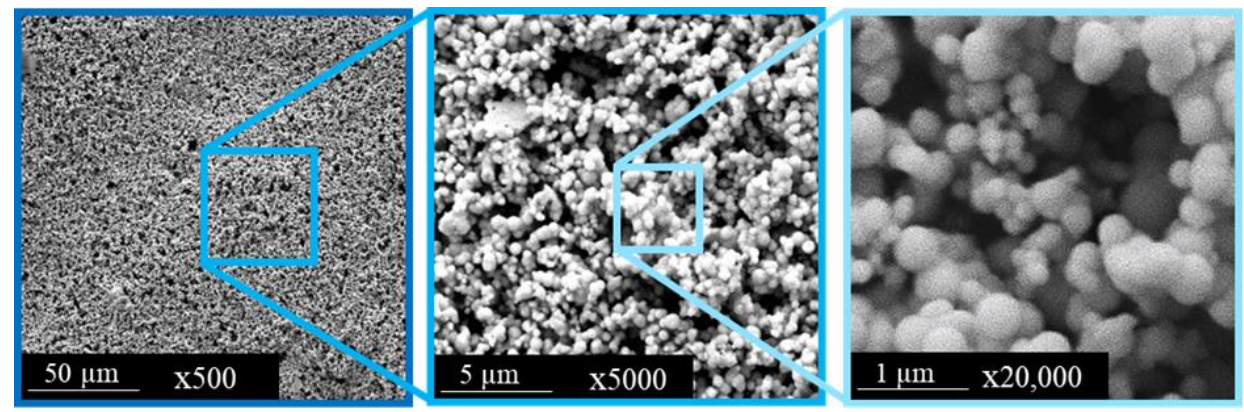

Figure 6.8. SEM micrographs of the working SPPtE at three different magnifications.

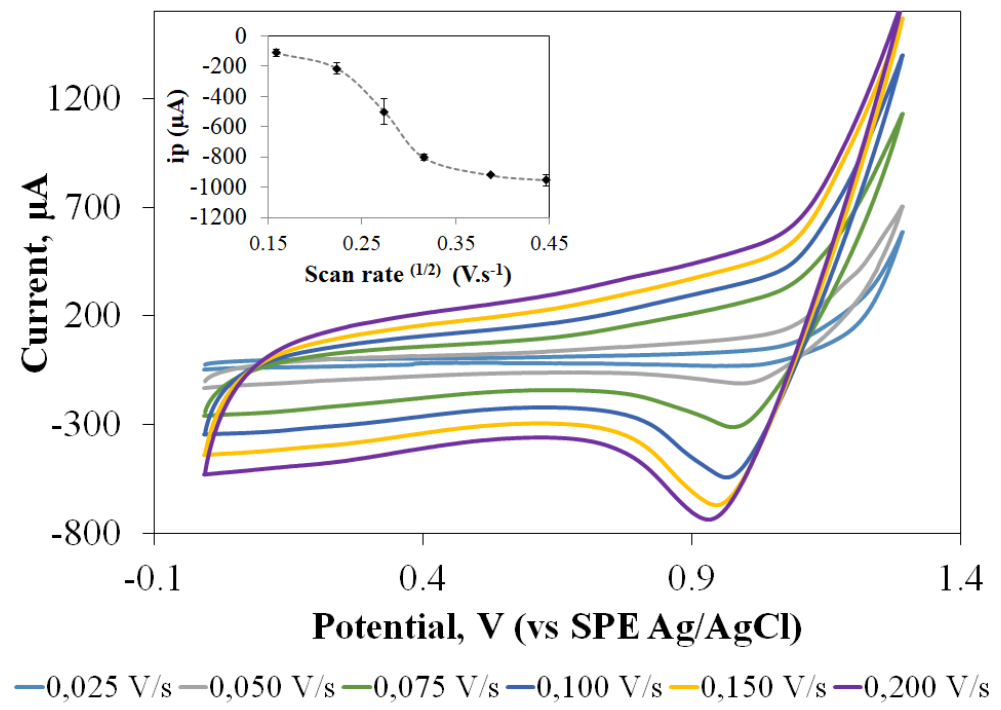

Figure 6.9. CV scans obtained from Randles-Sevcik study at SPPtE, of $200 \mu L$ of supporting electrolyte containing $60 \mathrm{mM} \mathrm{NaCl}$, recorded from $+0.40 \mathrm{~V}$ to $+1.30 \mathrm{~V}$. Scan rate from 0.025 , $0.050,0.075,0.100,0.150$ and $0.200 \mathrm{~V} \mathrm{~s}^{-1}$. Inset of square root of scan rate dependence of peak current. Error bars of peak current obtained $(n=3)$. 


\subsubsection{SPPtE-based sensor characterization}

If an analytical method is to be characterized, it is important to establish its precision in terms of reproducibility and repeatability. Reproducibility was calculated taking into account calibration curves registered by the different sensors (inter-sensors), and repeatability with one single sensor (intra-sensor). The CSV responses of the SPPtE sensor to chloride concentrations over a range of $150 \mathrm{mM}$ are shown in figures $6.10 \mathrm{~A}$ and $\mathrm{B}$. After $150 \mathrm{mM}$, a loss on linearity was observed, leading to saturation of the sensor. The average value of the voltammetric peak currents of several samples was used to construct the calibration curves. The reproducibility associated with the slopes of these calibrations, in terms of relative standard deviation (RSD) was $5.80 \%(n=7)$, and a sensitivity of -24.147 $\mu \mathrm{A} \mathrm{mM}^{-1}$ was achieved.
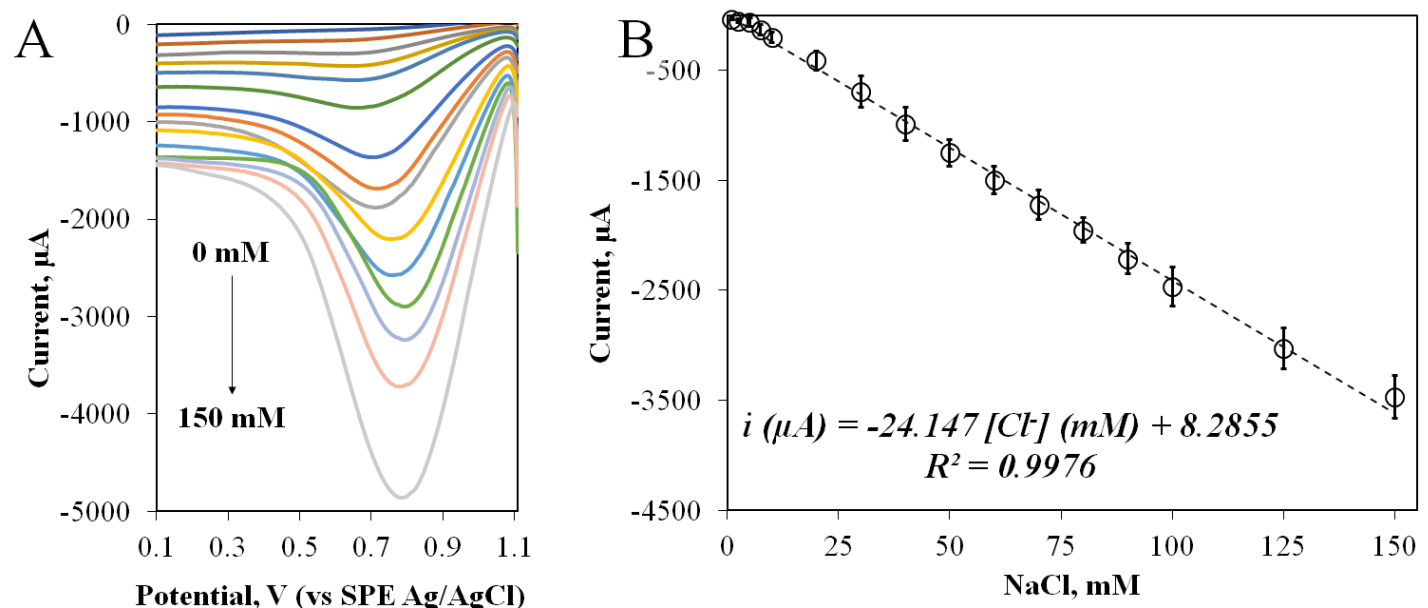

Figure 6.10. SPPtE sensor responses obtained in the optimal conditions. (a) CSV cathodic peaks recorded in a $200 \mu \mathrm{L}$ drop of supporting electrolyte with chloride ranging from 0 to $150 \mathrm{mM}$; and (b) Average calibration curve and associated deviation, registered for seven distinct SPPtEs.

In terms of repeatability, a single SPPtE-based sensor maintained a stable slope over six successive calibrations, each constructed of sixteen measurements (Figure 6.11). Despite 
the disposable characteristics of the SPEs, considering that the evaluation of each concentration corresponds to one experiment, each sensor was able to perform an average of $118 \pm 5$ reliable experiments in synthetic chloride samples. A limit of detection (LOD) of $0.76 \mathrm{mM}$, was obtained by LOD $=3 S_{x / y} / b$, where $S_{x / y}$ is the estimated standard deviation of the lowest concentration that was detected, and $b$ is the average slope of the calibration curves $[65,66]$.

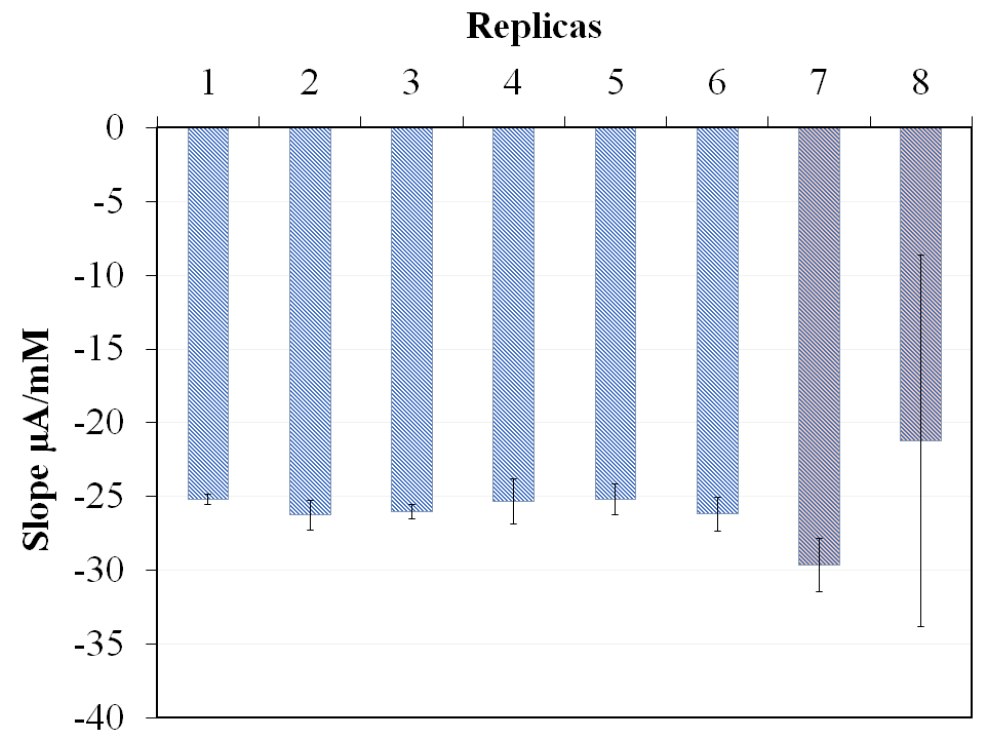

Figure 6.11. Study for SPPtE/EP based sensor slope stability under repeated measurements. Error bars corresponding to standard deviation of calibration slopes $(n=3)$.

\subsubsection{Analysis of interferences}

It is important to check the specificity of the SPPtE to chloride, when other halides are present at the same concentration levels. Therefore, fluoride, bromide and iodide were evaluated and their voltammetric behaviors were analyzed. Only bromide originated a reduction peak at $+0.60 \mathrm{~V}$ under the optimized experimental conditions. This reduction peak can hinder the chloride peak when bromide is present in concentrations above $10 \mathrm{mM}$. So, despite the different peak potential, the determination of chloride by using the SPPtEbased sensor in high bromide content samples should be reconsidered, in view of the false 
positive results that can emerge. Nonetheless, bromide is not expected to occur in such high concentrations in most samples.

Moreover, other anions and several cations, organic acids, carbohydrates, amino acids and nitrogen compounds, found in biological samples, were carefully analyzed at three distinct concentrations (Table 6.1). None of the components under consideration, showed a major interference with the sensor operation for chloride determination. However, biological matrices present various organic substances that can cause electrode passivation, reducing the availability of sites for the formation of chloride surface complexes.

\subsubsection{SPPtE-based sensor application and validation}

The SPPtE-based sensor can be a useful tool to determine chloride content in several matrices. It offers reliable performance for selective analysis with an absence of any relevant interferents. Efficient determination of this anion in environmental, pharmaceutical, and food samples was successfully performed. Some of these samples required a pretreatment step, such as centrifugation, dilution and calcination, in order to fit the sample into the calibration range, or as previously mentioned, to avoid electrode poisoning (Table 6.3).

Real sample analysis was performed using the standard addition method, except in the analysis of human sweat, the peak current value of which was introduced in the calibration curves previously built from synthetic sweat, given the limited availability of real samples.

The SPPtE sensor results, shown in table 6.4, corroborated with potentiometric measurements of chloride-ion selective electrode (Cl-ISE), confirmed the viability and applicability of the voltammetric sensor to determine the chloride content of several sample types. 
Table 6.3. Real samples pretreatment.

\begin{tabular}{cc}
\hline Samples & Pretreatment \\
\hline Seawater & Dilution \\
Saline Solution & Dilution \\
& $\begin{array}{c}15 \mathrm{~g} \text { of biscuits were minced and calcined for } 15 \mathrm{~min} \text { at } 400{ }^{\circ} \mathrm{C} \text {. The } \\
\text { resulting sample was dissolved in } 500 \mathrm{~mL} \text { of Milli-Q water. Finally, } \\
\text { the sample was filtered and centrifuged to remove any particles in } \\
\text { Sholemeal biscuits }\end{array}$ \\
$\begin{array}{c}\text { Cuspension. } \\
\text { Chicken Stock }\end{array}$ & Spiked with $47 \mathrm{mM}$ and $125 \mathrm{mM}$ of sodium chloride. \\
Urine & Dilution \\
Real Human Sweat & Untreated \\
\hline
\end{tabular}

Table 6.4. Chloride determination in different samples using potentiometric (Cl-ISE) and voltammetric (SPPtE) methods. Triplicates were performed for each sample analysis.

\begin{tabular}{ccccc}
\hline Samples & $\begin{array}{c}\boldsymbol{C l}-\boldsymbol{I S E} \\
{[\boldsymbol{C l}] \boldsymbol{m} \boldsymbol{M}}\end{array}$ & $\begin{array}{c}\boldsymbol{S P P t} \boldsymbol{E} \\
{[\boldsymbol{C l}] \boldsymbol{m} \boldsymbol{M}}\end{array}$ \\
\hline Seawater & 613.15 & \pm 6.77 & 613.14 & \pm 9.66 \\
Saline Solution & 141.10 & \pm 3.10 & 141.35 & \pm 3.46 \\
Wholemeal biscuits & 44.29 & \pm 1.77 & 47.11 & \pm 2.76 \\
Chicken Stock & 134.00 & \pm 1.16 & 122.36 & \pm 10.10 \\
& 46.37 & \pm 1.41 & 47.13 & \pm 0.53 \\
Human Plasma & 126.98 & \pm 2.26 & 126.05 & \pm 4.10 \\
Urine & 63.67 & \pm 2.89 & 60.81 & \pm 4.88 \\
Real Human Sweat & 31.92 & \pm 2.93 & 30.76 & \pm 3.61 \\
\hline
\end{tabular}




\subsubsection{Sensor application for sweat analysis}

Over the past few years, the application of sensorial platforms specifically for the determination of sweat chloride has been under investigation, for noninvasive analysis of this electrolyte [29,34,35,38], which is useful in CF disease diagnosis and for monitoring electrolyte loss during exercise [6-12]. Thus, considering the suitability of the sensor in this field, special attention was given to sweat samples.

In vitro studies, using synthetic sweat samples, shown in figure 6.12, yielded well defined CSV peaks showing excellent linear correlation with chloride concentrations $\left(\mathrm{R}^{2}=0.9997\right)$, and a sensitivity of $-13.618 \mu \mathrm{AmM}^{-1}$. Moreover, the electrodic system was printed on Gore-Tex ${ }^{\circledR}$, which could be considered as a proof-of-concept for sweat analysis, given that flexible substrates are required for the development of wearable devices. This device presented good linearity and analogous performances to those obtained with the rigid substrate.

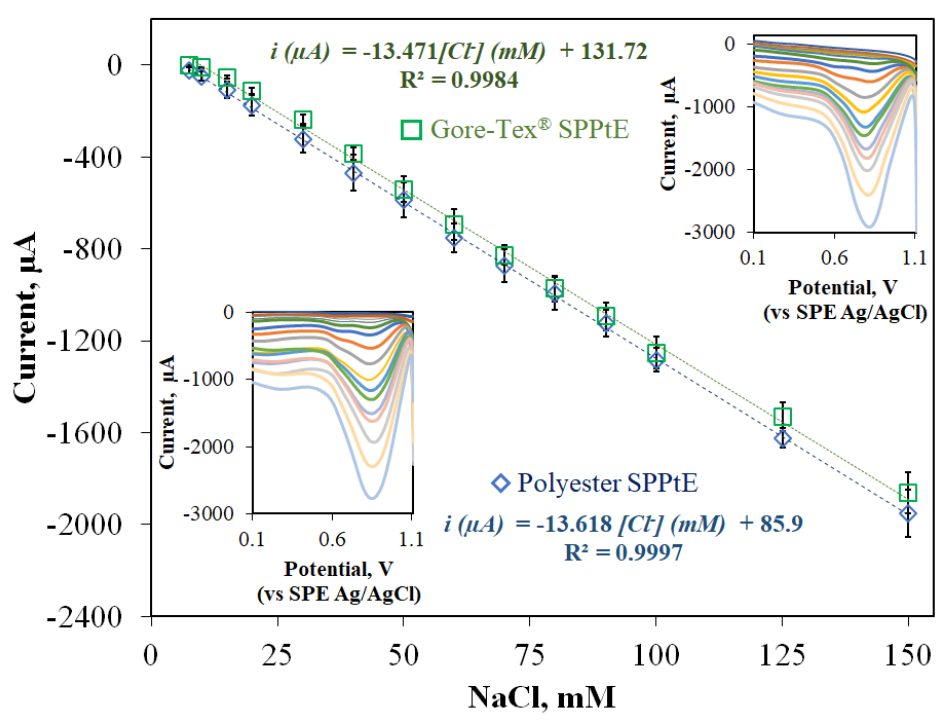

Figure 6.12. Flexible Gore-Tex ${ }^{\circledR}$ SPPtEs-based sensors voltammetric peaks recorded in a $200 \mu \mathrm{L}$ drop of synthetic sweat with chloride ranging from 0 to $150 \mathrm{mM}$, and respective average calibration curves for $(\diamond)$ rigid polyester SPPtE and $(\square)$ Flexible Gore-Tex ${ }^{\circledR}$ SPPtEs $(n=3)$. 
Comparing the CSV peaks for synthetic sweat with those of the supporting electrolyte (Figure 6.10), a lower slope of the calibration curves was found. These data may be an indicator of electrode poisoning, that agree with the work of Polta and Johnson, who observed that electrochemical detection of amino acids and carbohydrates was not successful using Pt electrodes, due to the accumulation of adsorbed reaction products under anodic potentials at Pt surfaces [67]. Nevertheless, a good linear relation was obtained in the range of interest to determine chloride concentration in real human sweat samples. Thus, the height of the reduction peaks was interpolated in the previously built synthetic sweat calibration curve, detecting a chloride value of $30.76 \pm 3.61 \mathrm{mM}$ in the human sweat sample, which agrees with the value obtained using the Cl-ISE (Table 6.4).

\subsection{CONCLUSIONS}

A new electrochemical chloride sensor has been developed, based on recognized interactions between the chloride anion and the platinum electrode surfaces. The formation of surface chloro-complexes at the SPPtE working electrode surface are oxidized in a primary anodic step to chlorine gas. This gas is strongly adsorbed onto the Pt surface and reduced in the cathodic scan, originating reduction peaks that present a linear relation with chloride concentration in the matrix. Its large linear range $(0.76$ to $150 \mathrm{mM})$ fits ideally with the chloride concentrations that can be found in several types of samples, making this sensor a versatile analytical tool. The device has proven to be a low-cost, noninvasive, simple, reusable and reproducible method for chloride determination. Furthermore, the high specificity of its analysis has revealed that the reaction between platinum and chloride prevails in the presence of numerous compounds, allowing selective chloride determination in different types of environmental, pharmaceutical, and food samples. Additionally, the printing technique is easily transferable to a flexible Gore-Tex ${ }^{\circledR}$ SPPtE-based sensor that future research could further adapt, as a wearable noninvasive tool for CF diagnosis and for monitoring electrolyte loss in endurance activities. 


\subsection{REFERENCES}

[1] J.A. Hern, G.K. Rutherford, G.W. Vanloon, Determination of chloride, nitrate, sulphate and total sulphur in environmental samples by single-column ion chromatography, Talanta. 30 (1983) 677-682.

[2] F.J. Millero, R. Feistel, D.G. Wright, T.J. McDougall, The composition of Standard Seawater and the definition of the Reference-Composition Salinity Scale, Deep. Res. Part I Oceanogr. Res. Pap. 55 (2008) 50-72.

[3] J.A. Morales, L.S. De Graterol, J. Mesa, Determination of chloride, sulfate and nitrate in groundwater samples by ion chromatography, J. Chromatogr. A. 884 (2000) 185-190.

[4] A.C. Galvis-Sánchez, I. V. Tóth, A. Portela, I. Delgadillo, A.O.S.S. Rangel, Monitoring sodium chloride during cod fish desalting process by flow injection spectrometry and infrared spectroscopy, Food Control. 22 (2011) 277-282.

[5] R. Pérez-Olmos, R. Herrero, J.L.F.C. Lima, M.C.B.S.M. Montenegro, Sequential potentiometric determination of chloride and nitrate in meat products, Food Chem. 59 (1997) 305-311.

[6] P.M. Farrell, T.B. White, N. Derichs, C. Castellani, B.J. Rosenstein, Cystic Fibrosis Diagnostic Challenges over 4 Decades: Historical Perspectives and Lessons Learned, J. Pediatr. 181 (2017) S16-S26.

[7] M.I. Barrio Gómez de Agüero, G. García Hernández, S. Gartner, Protocolo de diagnóstico y seguimiento de los pacientes con fibrosis quística, An. Españoles Pediatr. 50 (1999) 625-634. 
[8] V.A. LeGrys, J.R. Yankaskas, L.M. Quittell, B.C. Marshall, P.J. Mogayzel, Diagnostic Sweat Testing: The Cystic Fibrosis Foundation Guidelines, J. Pediatr. 151 (2007) 85-89.

[9] M.D. Robert C. Stern, The diagnosis of cystic fibrosis, N. Engl. J. Med. 336 (1997) $487-491$.

[10] B.J. Rosenstein, G.R. Cutting, The diagnosis of cystic fibrosis: A consensus statement, J. Pediatr. 132 (1998) 589-595.

[11] P.R. Sosnay, T.B. White, P.M. Farrell, C.L. Ren, N. Derichs, M.S. Howenstine, J.A. Nick, K. De Boeck, Diagnosis of Cystic Fibrosis in Nonscreened Populations, J. Pediatr. 181 (2017) S52-S57.

[12] W.A. Latzka, S.J. Montain, Water and electrolyte requirements for exercise, Clin. Sports Med. 18 (1999) 513-524.

[13] A.B. Stefaniak, C.J. Harvey, Dissolution of materials in artificial skin surface film liquids, Toxicol. Vitr. 20 (2006) 1265-1283.

[14] G.T. Nagami, Hyperchloremia - Why and how, Nefrologia. 36 (2016) 347-353.

[15] G. Morrison, Serum Chloride., in: H.K. Walker, W.D. Hall, J.W. Hurst (Eds.), Clin. Methods Hist. Phys. Lab. Exam., 3rd ed., Boston: Butterworths, 1990: pp. 890-894.

[16] W.P. Mutter, C.A. Korzelius, Urine Chemistries, Hosp. Med. Clin. 1 (2012) e338e352.

[17] R.W. Schrier, Diagnostic Value of Urinary Sodium, Chloride, Urea, and Flow, J. Am. Soc. Nephrol. 22 (2011) 1610-1613.

[18] S. Watanabe, T. Kimura, K. Suenaga, S. Wada, K. Tsuda, S. Kasama, T. Takaoka, K. Kajiyama, M. Takeda, H. Yoshikawa, Decreased chloride levels of cerebrospinal fluid in patients with amyotrophic lateral sclerosis, J. Neurol. Sci. 285 (2009) 146-148. 
[19] H.W. Doughty, Mohr's method for the determination of silver and halogens in other than neutral solutions, J. Am. Chem. Soc. 46 (1924) 2707-2709.

[20] P. Díaz, Z. González, M. Granda, R. Menéndez, R. Santamaría, C. Blanco, Evaluating capacitive deionization for water desalination by direct determination of chloride ions, Desalination. 344 (2014) 396-401.

[21] R.G. Du, R.G. Hu, R.S. Huang, C.J. Lin, In situ measurement of $\mathrm{Cl}^{-}$concentrations and $\mathrm{pH}$ at the reinforcing steel/concrete interface by combination sensors, Anal. Chem. 78 (2006) 3179-3185.

[22] W.T. Grubb, Chloride - selective electrode, US3740326, 1973.

[23] A. Hulanicki, A. Michalska, All-solid-state chloride-selective electrode with poly(pyrrole) solid contact, Electroanalysis. 7 (1995) 692-693.

[24] J.S.F. Pereira, L.O. Diehl, F.A. Duarte, M.F.P. Santos, R.C.L. Guimarães, V.L. Dressler, É.M.M. Flores, Chloride determination by ion chromatography in petroleum coke after digestion by microwave-induced combustion, J. Chromatogr. A. 1213 (2008) 249252.

[25] A.M. Pimenta, A.N. Araújo, M.C.B.S.M. Montenegro, C. Pasquini, J.J.R. Rohwedder, I.M. Raimundo, Chloride-selective membrane electrodes and optodes based on an indium (III) porphyrin for the determination of chloride in a sequential injection analysis system, J. Pharm. Biomed. Anal. 36 (2004) 49-55.

[26] H. Cao, D.H. Wu, Rapid and sensitive determination of trace chloride ion in drinks using resonance light scattering technique, J. Autom. Methods Manag. Chem. 2008 (2008) $0-5$.

[27] R.B. Mesquita, S.M. Fernandes, A.O. Rangel, Turbidimetric determination of chloride in different types of water using a single sequential injection analysis system, $\mathbf{J}$ Env. Monit. 4 (2002) 458-461. 
[28] M.O. Gorbunova, A.V. Shevchenko, V.V. Apyari, A.A. Furletov, P.A. Volkov, A.V. Garshev, S.G. Dmitrienko, Selective determination of chloride ions using silver triangular nanoplates and dynamic gas extraction, Sensors Actuators, B Chem. 256 (2018) 699-705.

[29] A. Koh, D. Kang, Y. Xue, S. Lee, R.M. Pielak, J. Kim, T. Hwang, S. Min, A. Banks, M.C. Manco, L. Wang, K.R. Ammann, K. Jang, S. Han, R. Ghaffari, U. Paik, M.J. Slepian, Y. Huang, J.A. Rogers, A Soft, Wearable Microfluidic Device for the Capture, Storage, and Colorimetric Sensing of Sweat, Sci Transl Med. 8 (2017).

[30] A. Martin, R. Narayanaswamy, Studies on quenching of fluorescence of reagents in aqueous solution leading to an optical chloride-ion sensor, Sensors Actuators B Chem. 39 (1997) 330-333.

[31] V.G. Bonifácio, L.C. Figueiredo-Filho, L.H. Marcolino, O. Fatibello-Filho, An improved flow system for chloride determination in natural waters exploiting solid-phase reactor and long pathlength spectrophotometry, Talanta. 72 (2007) 663-667.

[32] L.S. Laxmeshwar, M.S. Jadhav, J.F. Akki, P. Raikar, J. Kumar, O. prakash, R. Mahakud, U.S. Raikar, Quantification of chloride and iron in sugar factory effluent using long period fiber grating chemical sensor, Sensors Actuators B Chem. 258 (2018) 850-856.

[33] D.B. de Graaf, Y. Abbas, J.G. Bomer, W. Olthuis, A. van den Berg, Sensor-actuator system for dynamic chloride ion determination, Anal. Chim. Acta. 888 (2015) 44-51.

[34] D.H. Choi, Y. Li, G.R. Cutting, P.C. Searson, A wearable potentiometric sensor with integrated salt bridge for sweat chloride measurement, Sensors Actuators, B Chem. 250 (2017) 673-678.

[35] J. Bujes-Garrido, M.J. Arcos-Martínez, Development of a wearable electrochemical sensor for voltammetric determination of chloride ions, Sensors Actuators, B Chem. 240 (2017) 224-228. 
[36] A. Cranny, N. Harris, N. White, Screen printed potentiometric chloride sensors, Procedia Eng. 87 (2014) 220-223.

[37] V.A.T. Dam, M.A.G. Zevenbergen, R. Van Schaijk, Toward wearable patch for sweat analysis, Sensors Actuators, B Chem. 236 (2016) 834-838.

[38] V.A.T. Dam, M.A.G. Zevenbergen, R. Van Schaijk, Flexible chloride sensor for sweat analysis, Procedia Eng. 120 (2015) 237-240.

[39] V.A.T. Dam, M. Goedbloed, M.A.G. Zevenbergen, Solid-Contact Reference Electrode for Ion-Selective Sensors, Proceedings. 1 (2017) 464.

[40] I. Campos, R. Masot, M. Alcañiz, L. Gil, J. Soto, J.L. Vivancos, E. García-Breijo, R.H. Labrador, J.M. Barat, R. Martínez-Mañez, Accurate concentration determination of anions nitrate, nitrite and chloride in minced meat using a voltammetric electronic tongue, Sensors Actuators, B Chem. 149 (2010) 71-78.

[41] A.J. Bandodkar, J. Wang, Non-invasive wearable electrochemical sensors: A review, Trends Biotechnol. 32 (2014) 363-371.

[42] M. Bariya, H.Y.Y. Nyein, A. Javey, Wearable sweat sensors, Nat. Electron. 1 (2018) $160-171$.

[43] S. Emaminejad, W. Gao, E. Wu, Z.A. Davies, H. Yin Yin Nyein, S. Challa, S.P. Ryan, H.M. Fahad, K. Chen, Z. Shahpar, S. Talebi, C. Milla, A. Javey, R.W. Davis, Autonomous sweat extraction and analysis applied to cystic fibrosis and glucose monitoring using a fully integrated wearable platform, Proc. Natl. Acad. Sci. 114 (2017) 4625-4630.

[44] W. Gao, S. Emaminejad, H.Y.Y. Nyein, S. Challa, K. Chen, A. Peck, H.M. Fahad, H. Ota, H. Shiraki, D. Kiriya, D.-H. Lien, G.A. Brooks, R.W. Davis, A. Javey, Fully integrated wearable sensor arrays for multiplexed in situ perspiration analysis, Nature. 529 (2016) 509-514. 
[45] G. Matzeu, L. Florea, D. Diamond, Advances in wearable chemical sensor design for monitoring biological fluids, Sensors Actuators, B Chem. 211 (2015) 403-418.

[46] J.R. Windmiller, J. Wang, Wearable Electrochemical Sensors and Biosensors: A Review, Electroanalysis. 25 (2013) 29-46.

[47] M.H. Chiu, W.L. Cheng, G. Muthuraman, C.T. Hsu, H.H. Chung, J.M. Zen, A disposable screen-printed silver strip sensor for single drop analysis of halide in biological samples, Biosens. Bioelectron. 24 (2009) 3008-3013.

[48] J. Bujes-Garrido, M.J. Arcos-Martínez, Disposable sensor for electrochemical determination of chloride ions, Talanta. 155 (2016) 153-157.

[49] O.D. Renedo, M.A. Alonso-Lomillo, M.J.A. Martínez, Recent developments in the field of screen-printed electrodes and their related applications, Talanta. 73 (2007) 202219.

[50] J. Bujes-Garrido, D. Izquierdo-Bote, A. Heras, A. Colina, M.J. Arcos-Martínez, Determination of halides using Ag nanoparticles-modified disposable electrodes. A first approach to a wearable sensor for quantification of chloride ions, Anal. Chim. Acta. 1012 (2018) 42-48.

[51] A.T. Kuhn, P.M. Wright, The behaviour of platinum, iridium and ruthenium electrodes in strong chloride solutions, J. Electroanal. Chem. 41 (1973) 329-349.

[52] S.B. Hall, E.A. Khudaish, A.L. Hart, Electrochemical oxidation of hydrogen peroxide at platinum electrodes. Part V: inhibition by chloride, Electrochim. Acta. 45 (2000) 35733579 .

[53] J. Gulens, Surface effects in relation to the response of solid-state ion-selective eletrodes, Ion-Selective Electrode Rev. 2 (1981) 117-157. 
[54] J. Clavilier, The role of anion on the electrochemical behaviour of a $\{111\}$ platinum surface; an unusual splitting of the voltammogram in the hydrogen region, J. Electroanal. Chem. 107 (1980) 211-216.

[55] J.A. Polta, D.C. Johnson, Pulsed Amperometric Detection of Electroinactive Adsorbates at Platinum Electrodes in a Flow Injection System, Anal. Chem. 57 (1985) $1373-1376$.

[56] N. Priyantha, S. Malavipathirana, Effect of chloride irons on the electrochemical behaviour of platinum surfaces, J. Natl. Sci. Found. Sri Lanka. 24 (1996) 237-246.

[57] E.L. Littauer, L.L. Shreir, Anodic polarization of platinum in sodium chloride solutions, Electrochim. Acta. 11 (1966) 527-536.

[58] X.J. Huang, D.S. Silvester, I. Streeter, L. Aldous, C. Hardacre, R.G. Compton, Electroreduction of chlorine gas at platinum electrodes in several room temperature ionic liquids: Evidence of strong adsorption on the electrode surface revealed by unusual voltammetry in which currents decrease with increasing voltage scan rates, J. Phys. Chem. C. 112 (2008) 19477-19483.

[59] L. Sereno, V.A. Macagno, M.C. Giordano, Electrochemical Behaviour of the Chloride / Chlorine System at Platinum Electrodes in Acetonitrile Solutions, Electrochim. Acta. 17 (1972) 561-575.

[60] K. Murugappan, D.W.M. Arrigan, D.S. Silvester, Electrochemical Behavior of Chlorine on Platinum Microdisk and Screen-Printed Electrodes in a Room Temperature Ionic Liquid, J. Phys. Chem. C. 119 (2015) 23572-23579.

[61] C.J. Harvey, R.F. LeBouf, A.B. Stefaniak, Formulation and stability of a novel artificial sebum under conditions of storage and use, Int. J. Cosmet. Sci. 32 (2010) 347355. 
[62] B. Molinero-Abad, D. Izquierdo, L. Pérez, I. Escudero, M.J. Arcos-Martínez, Comparison of backing materials of screen printed electrochemical sensors for direct determination of the sub-nanomolar concentration of lead in seawater, Talanta. 182 (2018) $549-557$.

[63] P. Daubinger, J. Kieninger, T. Unmüssig, G.A. Urban, Electrochemical characteristics of nanostructured platinum electrodes - a cyclic voltammetry study, Phys. Chem. Chem. Phys. 16 (2014) 8392-9.

[64] A.J. Bard, L.R. Faulkner, Electrochemical Methods - Fundamentals and Applications, 2nd Ed., John Wiley \& Sons, Inc., 2001.

[65] J. Mocak, A. M. Bond, S. Mitchell, G. Scollary, A statistical overview of standard (IUPAC and ACS) and new procedures for determining the limits of detection and quantification: Application to voltammetric and stripping techniques (Technical Report), Pure Appl. Chem. 69 (1997) 297-328.

[66] E.R. Ziegel, Statistics and Chemometrics for Analytical Chemistry, 2004.

[67] J.A. Polta, D.C. Johnson, The Direct Electrochemical Detection of Amino Acids at a $\mathrm{Cu}$ Electrode in an Acidic Chromatographic Effluent, J. Liq. Chromatogr. 6 (1983) 17271743. 



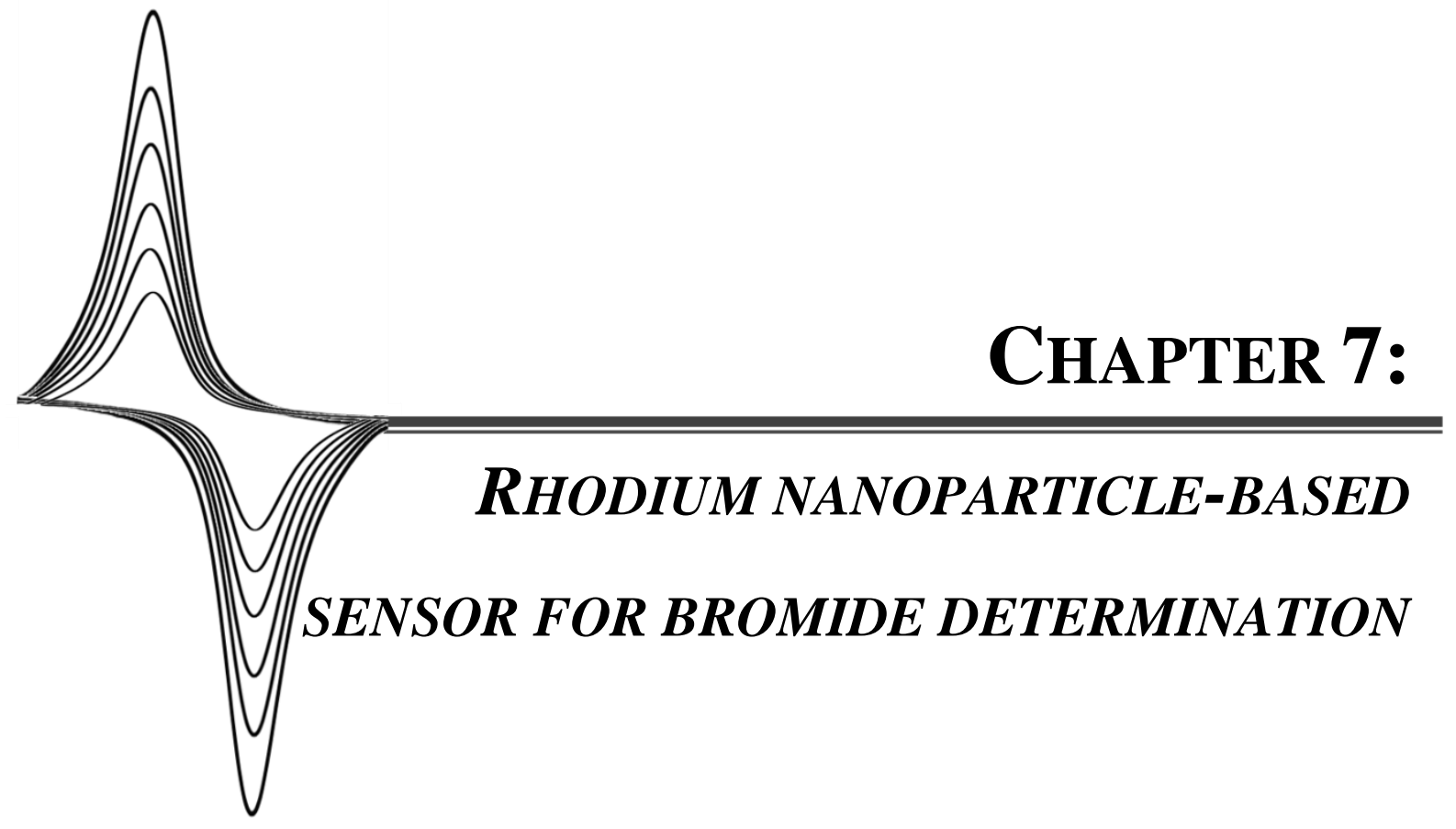



Although it was not problematic for the sample analysis performed in the previous work, the possibility of false positive resultas due to the presence of high bromide levels, lead us to make use of this behavior as the base for a new bromide sensor.

Present in different applications, the demand for bromide monitoring, has increased over recent years. Especially attention in sources of bromide contamination, such are the brines from hydraulic fracturing, pesticides and brominated polymers. The presence of bromide anions leads to the formation of bromide disinfection byproducts during the treatment of waters.

Based in the reduction of bromine, previously generated at anodic potentials, the cathodic signal obtained with the proposed sensor, can be related with the initial anion concentration in the sample. The interference of chloride anions was avoided using rhodium nanoparticles (Rh-Nps), which offers higher selectivity for bromide.

A single drop of the sample on sensor surface is adequate for the determination of bromide, carried out through the measurement of voltammetric cathodic peaks' current. This disposable Rh/SPCE-based sensor offers a detection limit of $39 \mu \mathrm{M}$, with a calibration range up to $40 \mathrm{mM}$ and a sensitivity of $23.28 \mu \mathrm{A} \mathrm{mM}^{-1}$. Its application to real-life samples evidenced recovery values close to $100 \%$, demonstrating the powerful analytical performance of the proposed method.

This work has been published in Sensors and Actuators B: Chemical 282, 2019, p. 603608. 



\subsection{INTRODUCTION}

Bromide has been extensively applied in several fields and is present in pesticides, medicines, industrial solvents, gasoline additives, and water purification compounds [1]. This halide anion was first used in medicine as potassium bromide in the $19^{\text {th }}$ century, when applied in the treatment of epilepsy, and as both a sedative and an anxiolytic [2]. It remains in use in clinical veterinary, due to its antiepileptic properties [3,4]. Several other pharmaceuticals contain bromide concentrations, which make this anion an important parameter to control in formulations [2-5]. Moreover, this halide properties have also been applied to polymeric materials, particularly for fireproofing. Brominated flame retardants consist of polymer additives, employed in the production of electronic devices, clothing, furniture, and electrical appliances. Issues relating to brominated polymers emerged from the weakly bond characteristic of this additive, making it highly susceptible to be released into the environment. Actually, current regulations restrict the use of organobrominated compounds, categorizing them as persistent pollutants, due to their potential bioaccumulation in ecosystems and their toxic effects on wildlife [6].

Further importance has been given to its use in hydrogeology, where bromide can occur naturally or can be artificially introduced as a tracer to track subsurface water flows. Wastes and unused residues from the use of this halide have resulted in widespread ground water contamination [1]. Additional sources of bromide contamination are the brines generated from oil and natural gas production. Flowback and produced waters from hydraulic fracturing of shale gas are recognized to contain high concentrations of bromides ( 12 $\mathrm{mM})$. A major concern with these brines is the formation of brominated disinfection byproducts, especially problematic for the treatment of potable water $[7,8]$.

Bromide as an analyte was not of great interest until the last decade and there are very few papers on its determination. Approaches based on volumetric techniques [9], spectrophotometry [10,11], colorimetry [12], ion-exchange chromatography [13,14], 
molecular absorption spectrometry [6], and neutron activated analysis [11], have been used to determine bromide content in several types of samples. Despite the fragility of the electrodes and the need for sample preparation, bromide-ion selective electrodes have proven their simplicity and selectivity in the potentiometric determination of the anion [1517].

The current conflict between economic and environmental interests demands the control of brominated species, which has led to the development of alternative electrochemical approaches. Benett et al. [18] performed an electrochemical study of the voltammetric behavior of the redox pair $\mathrm{Br}^{-} / \mathrm{Br}_{2}$ in non-aqueous solvents. Other approaches, such as wastewater treatment, were designed to allow the selective oxidation of bromide in brines from hydraulic fracturing [7]. Also, the use of silver electrodes has permitted the study of halide reactions onto the electrode surface [19]. More recently, the great advantages of screen-printed electrodes (SPE) have been recognized, mainly due to the great adaptability of these traducers, their portability for in situ analysis, as well as their low-cost, disposability and reproducibility. The easy handling and the wide range of possibilities for SPE modifications, contributes as significant improvements to this sensing platforms [20]. Silver SPE [21] and the modification of SPEs with silver nanoparticles [22] have proved to be suitable for bromide quantification in real samples.

Besides silver, several types of metallic electrodeposition nanostructures have been successfully accomplished in numerous SPE systems, offering unique advantages in terms of sensitivity, selectivity, working stability and electroactive surface area increase [22-27]. Rhodium nanoparticles (Rh-Nps) are the least represented among the nanoparticle-based electrochemical sensors. Nevertheless, the utility of this nanosized Rh has improved the performances of various electrochemical devices [28-31].

Only few works regarding the electrochemical determination of bromide were found (Table 7.1), revealing a research gap in the determination of this anion. The increased interest in determining bromide led us to develop a new, sensitive, selective, rapid, disposable, lowcost, and easy-to-use method for in situ analysis of this halide. 
Thus, the direct reduction of bromine to bromide, oxidized in a previous anodic step, is investigated in this manuscript. The working carbon SPE modification with Rh-Nps, together with the presence of a chloride supporting electrolyte, allowed to achieve an efficient method for the determination of a wide concentration range of the interest halide. Hence, the successful application of the sensor to seawater, used to simulate the brines from hydraulic fracturing, showed suitable recovery values ( 100\%). Additionally, the satisfactory application of the method to a hexadecyltrimethylammonium bromide (CTAB) surfactant synthetic solution, provide a method for this tensioactive determination. Also, the bromide content of pharmaceutical formulations, such as dextromethorphan hydrobromide and ipratropium bromide, was correctly determined matching the sensor results with the labeled concentrations. 
Table 7.1. Comparison of analytical characteristics of various electrochemical sensing platforms for bromide determination.

\begin{tabular}{|c|c|c|c|c|c|c|c|}
\hline Electrodes & Techniques & Linear Range & $\begin{array}{c}L O D \\
(M)\end{array}$ & $\begin{array}{c}R S D \\
(\%)\end{array}$ & Samples & Observations & References \\
\hline $\mathrm{Br}-\mathrm{ISE} / \mathrm{PtE}$ & PD & $6.3 \times 10^{-7}-6.3 \times 10^{-4}$ & $6.3 \times 10^{-7}$ & NR & NR & $\begin{array}{l}\text { Complicate sample pretreatment. } \\
\text { Reduced range of analysis. } \\
\text { High volumes required. }\end{array}$ & [15] \\
\hline Br-ISE & PD & $1.0 \times 10^{-5}-3.5 \times 10^{-3}$ & $1.0 \times 10^{-5}$ & 1.70 & $\begin{array}{l}\text { CTAB critical micellar } \\
\text { concentration }\end{array}$ & $\begin{array}{l}\text { Complicate sample pretreatment. } \\
\text { High volumes required. }\end{array}$ & {$[16]$} \\
\hline CTSBr-ISE & PD & $1.3 \times 10^{-5}-6.0 \times 10^{-2}$ & $1.3 \times 10^{-5}$ & 1.59 & Soils & $\begin{array}{l}\text { Complicate mounting scheme and } \\
\text { sample pretreatment. }\end{array}$ & [17] \\
\hline SPAgE & LSV & $1.0 \times 10^{-5}-2.0 \times 10^{-2}$ & $3.0 \times 10^{-6}$ & 1.74 & Pond water & $\begin{array}{l}\text { Need of electrode pretreatment. } \\
\text { Low interference of } \mathrm{SCN}^{-} \text {. }\end{array}$ & [21] \\
\hline Ag-Nps/SPCE & LSV & $5.0 \times 10^{-6}-0.9 \times 10^{-4}$ & $5.0 \times 10^{-6}$ & 2.83 & NR & $\begin{array}{c}\text { Reduced range of analysis. } \\
\text { Single measurement electrodes. }\end{array}$ & {$[22]$} \\
\hline Rh-Nps/SPCE & $\mathrm{CSV}$ & $3.9 \times 10^{-5}-4.0 \times 10^{-2}$ & $3.9 \times 10^{-5}$ & 2.58 & $\begin{array}{l}\text { Seawater, CTAB } \\
\text { surfactant, } \\
\text { pharmaceuticals }\end{array}$ & $\begin{array}{l}\text { No sample pretreatment. Fast } \\
\text { analysis. Low iodide interference. }\end{array}$ & This work \\
\hline
\end{tabular}

Ag-Nps - Silver nanoparticles; Br-ISE - Commercial bromide-ion selective electrode; CTAB - hexadecyltrimethylammonium bromide surfactant; CTSBr-ISE

- Coated tubular solid-state bromide-ion selective electrode; CSV - Cathodic stripping voltammetry; LSV - Linear sweep voltammetry; NR - Not reported; PD

- Potentiometric detection; PtE - Platinum electrode; SPAgE - Screen-printed silver electrode; SPCE - Screen-printed carbon electrode; Rh-Nps - Rhodium nanoparticles. 


\subsection{EXPERIMENTAL SECTION}

\subsubsection{Chemicals and reagents}

Rhodium (III) chloride hydrate, potassium bromide, sodium chloride, potassium sulfate, sodium phosphate dibasic dihydrate, sodium phosphate monobasic dihydrate, potassium nitrate, sodium carbonate, methanol, and sulfuric acid, were obtained from Merck (Darmstadt, Germany). Sodium nitrite, potassium iodide, and cetyltrimethylammonium bromide (CTAB) were purchased from Sigma-Aldrich (Steinheim, Germany).

All reagents were of analytical grade and all solutions were prepared with Milli-Q water (Millipore, Bedford, USA).

Seawater samples were collected from Pontevedra coast, in the northwest of Spain. The samples were subsequently stored in high density polyethylene bottles in a freezer until the analysis. Dextromethorphan hydrobromide and ipratropium bromide commercial formulations were obtained from a local pharmacy.

The inks used for the fabrication of the screen-printed carbon electrodes (SPCE), Electrodag PF-407 A (carbon ink), Electrodag 6037 SS (silver/silver chloride ink), Electrodag 418 (silver ink) and Electrodag 452 SS (dielectric ink), were supplied by Achenson Colloiden (Scheemda, Netherlands). Polyester films (PET) of $0.5 \mathrm{~mm}$ thickness (HIFI Industrial Film, Dardily, France), were used as the printing substrates. The SPEs were fabricated as recently described elsewhere [26]. Briefly, a sequential deposition of the appropriate inks defines, the conductive silver tracks, the $\mathrm{Ag} / \mathrm{AgCl}$ pseudo-reference electrode ( $\mathrm{SPE} \mathrm{Ag} / \mathrm{AgCl})$, the carbon counter and working electrodes $\left(0.126 \mathrm{~cm}^{2}\right)$ and the insulating layer. 


\subsubsection{Apparatus}

Voltammetric measurements were performed using a PalmSens ${ }^{\circledR}$ portable electrochemical potentiostat with the PS Trace 4.2 program (PalmSens ${ }^{\circledR}$ Instruments BV, Houten, The Netherlands). Microscopy imaging was performed using a scanning electron microscope (SEM) JEOL JSM-6460LV (Abingdon-on-Thames, UK).

\subsubsection{Rhodium nanoparticles electrodeposition}

Electrodeposition of rhodium nanoparticles (Rh-Nps) was performed using chronoamperometric technique, by drop-casting $150 \mu \mathrm{L}$ of a solution of rhodium (III) chloride $(0.1 \mathrm{mM})$ in sulfuric acid $(0.5 \mathrm{M})$ onto the SPCE, and applying a potential of $0.25 \mathrm{~V}$ (vs SPE $\mathrm{Ag} / \mathrm{AgCl}$ ) for $480 \mathrm{~s}$. The electrode was then rinsed with water to prepare it for use in the experiment.

\subsubsection{Cyclic voltammetric measurements}

Cyclic voltammetric (CV) experiments for the electrochemical screening of bromide were performed, by drop-casting $150 \mu \mathrm{L}$ of phosphate buffer saline (PBS) (0.05 M, pH 7 and $0.05 \mathrm{M}$ of $\mathrm{NaCl}$ ), containing $0.5,1,2.5,5,10,20,40$ and $60 \mathrm{mM}$ of $\mathrm{KBr}$. Voltammograms were recorded between 0.00 and $+1.80 \mathrm{~V}(\mathrm{vs} \mathrm{SPE} \mathrm{Ag} / \mathrm{AgCl})$ at $0.10 \mathrm{~V} \mathrm{~s}^{-1}$.

\subsubsection{Cathodic stripping voltammetry (CSV) for bromide determination}

For bromide determination, $150 \mu \mathrm{L}$ of problem solution was drop-cast onto the sensor working area, and an anodic step of $+1.25 \mathrm{~V}$ (vs SPE $\mathrm{Ag} / \mathrm{AgCl}$ ) was applied over 20 seconds. Immediately after (equilibration time $=0 \mathrm{~s}$ ), a cathodic linear sweep voltammetry scan, from $+1.11 \mathrm{~V}$ to $-0.25 \mathrm{~V}$ (vs SPE $\mathrm{Ag} / \mathrm{AgCl}$ ) at a scan rate of $0.10 \mathrm{~V} \mathrm{~s}^{-1}$ was performed, returning a reduction peak at $+0.50 \mathrm{~V}$. The sensor was rinsed with Milli-Q water prior to subsequent measurements. 


\subsubsection{Methanolic extraction for dextromethorphan hydrobromide formulation}

The pharmaceutical formulation of dextromethorphan hydrobromide, due to the high viscosity of the matrix, required an additional step before the electrochemical measurement. A mixture of dextromethorphan hydrobromide and methanol (1:1) was stirred for 30 minutes at room temperature. The methanolic mixture was then diluted in supporting electrolyte (1:1) and measured following the indications in the previous section.

\subsection{RESULTS AND DISCUSSION}

\subsubsection{Bromide electrochemical behavior}

The cyclic voltammograms of different potassium bromide concentrations are shown in figure 7.1. The voltammograms revealed a quasi-reversible redox process, developing the corresponding oxidation and reduction peaks, at $+1.35 \mathrm{~V}$ and $+0.50 \mathrm{~V}$, respectively. The observed behavior is described in equations 7.1 and 7.2.

$$
\begin{aligned}
& 2 \mathrm{Br}^{-} \rightarrow \mathrm{Br}_{2}+2 e^{-}, \quad E_{\text {panodic }}=+1.35 \mathrm{~V} \\
& \mathrm{Br}_{2}+2 e^{-} \rightarrow 2 \mathrm{Br}^{-}, \quad E_{\text {p cathodic }}=+0.50 \mathrm{~V}
\end{aligned}
$$

Considering both processes, the oxidation of bromide requires very positive potentials for the reaction to take place, growing a peak in the buffer discharge zone. Thus, this oxidation process is more susceptible to peak deformation, as it is developed from a non-stable base line, which difficult the correct quantification of the electrochemical signals. In contrast, the reduction peak was developed at lower potentials (+0.50 V (vs SPE Ag/AgCl)), demonstrating a stable behavior and a good correlation with bromide concentrations. Accordingly, the cathodic process was selected for bromide determination. 


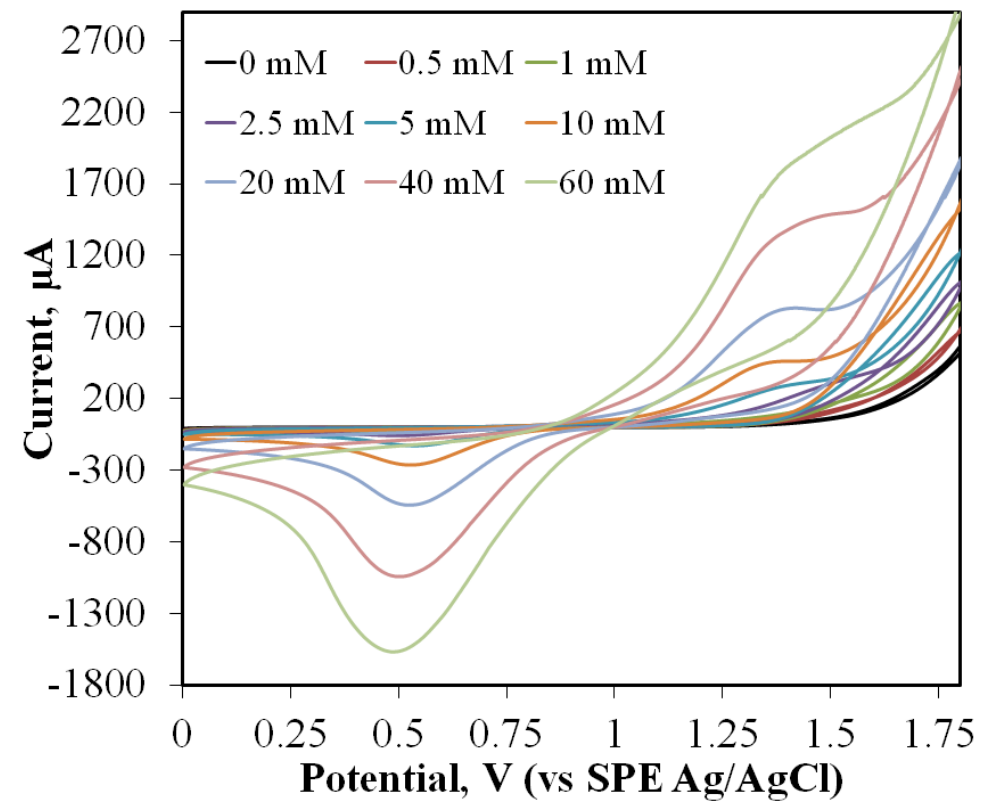

Figure 7.1. Multi-CV cycling for bromide determination, recorded at SPCE in a $150 \mu L$ drop containing distinct $\mathrm{KBr}$ concentrations in $\mathrm{PBS}(0.05 \mathrm{M}, \mathrm{pH}$ 7, and $0.01 \mathrm{M}$ of $\mathrm{NaCl})$. Scan rate 0.10 $V s^{-1}$.

\subsubsection{Bromide cathodic stripping voltammetric study}

Once checked the electrochemical behavior of bromide, cathodic stripping voltammetry (CSV) was selected as the technique for the study of the anion, since a quick analysis can be performed achieving well defined and stable peaks. In fact, the anodic step of CSV increased the bromide oxidation process (equation 7.1), thereby favoring bromine reduction (equation 7.2) in the cathodic step. However, the high potential required in the anodic step can be problematic in a real sample analysis, because several species can be oxidized that might interfere later with the bromide cathodic peaks. The potential and time of anodic stripping were therefore optimized, setting the CSV measurement at $+1.25 \mathrm{~V}$ during $20 \mathrm{~s}$ (Figure 7.2). 

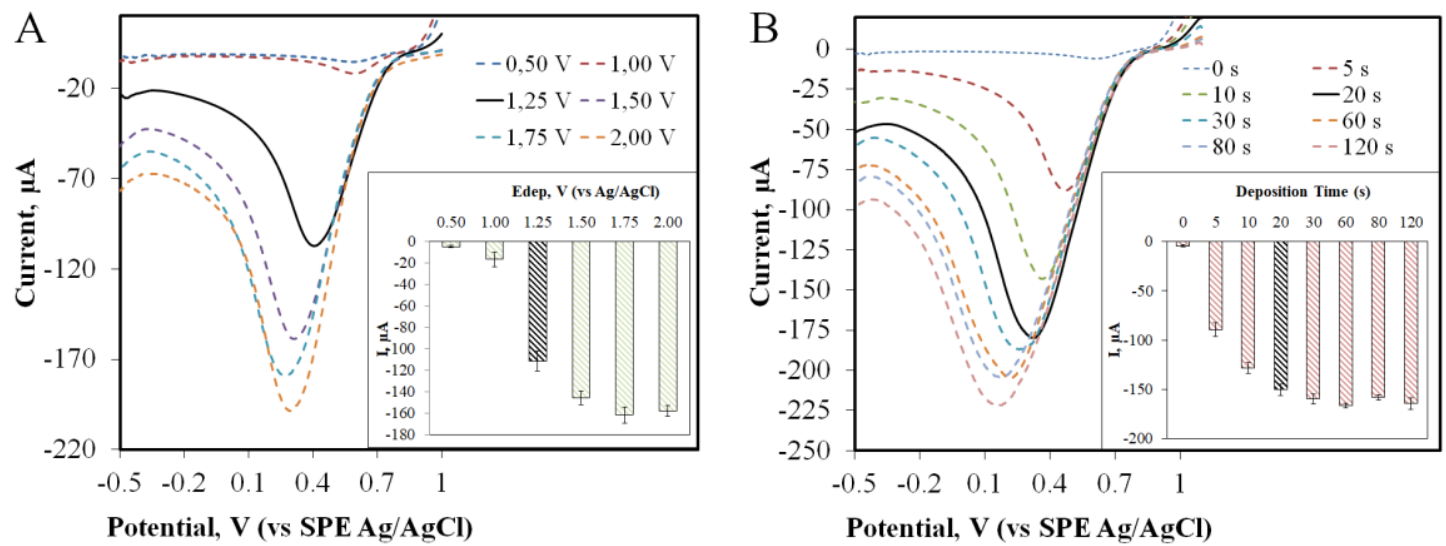

Figure 7.2. Voltammograms recorded from +1.11 to - $0.50 \mathrm{~V}$ at SPCE for $150 \mu \mathrm{L}$ of $5 \mathrm{mM}$ of $\mathrm{KBr}$ in PBS (0.05 M, pH 7, and 0.01 $\mathrm{M}$ of $\mathrm{NaCl})$ for: (A) Anodic potential of 0.50, 1.00, 1.25, 1.50 and $2.00 \mathrm{~V}$, applied for $10 \mathrm{~s}$; and (B) Anodic potential of $+1.25 \mathrm{~V}$, applied for 0, 5, 10, 15, 20, 30, 60, 80 and 120 s. Scan rate: $0.10 \mathrm{~V} \mathrm{~s}^{-1}(n=3)$.

\subsubsection{Rhodium nanoparticles electrodeposition}

The reduction of high bromide concentrations on the carbon working electrode originated well-defined peaks. However, the sensitivity required for several samples, where bromide can be found in the $\mu \mathrm{M}$ concentration range, was not achieved. Regarding that, it was found that smaller concentrations of bromide could be detected, by modifying the carbon surface with rhodium nanoparticles (Rh-Nps).

The Rh-Nps were generated by chronoamperometric measurements of a Rh solution dropcast onto the SPCE. For that, deposition potentials of $-0.80,-0.50,-0.25,-0.10,+0.18$ and $+0.25 \mathrm{~V}$ were applied during $480 \mathrm{~s}$, and the cathodic peak of bromide concentrations (lower than $1 \mathrm{mM}$ ), were compared with the one obtained in an unmodified SPCE (Figure 7.3). 


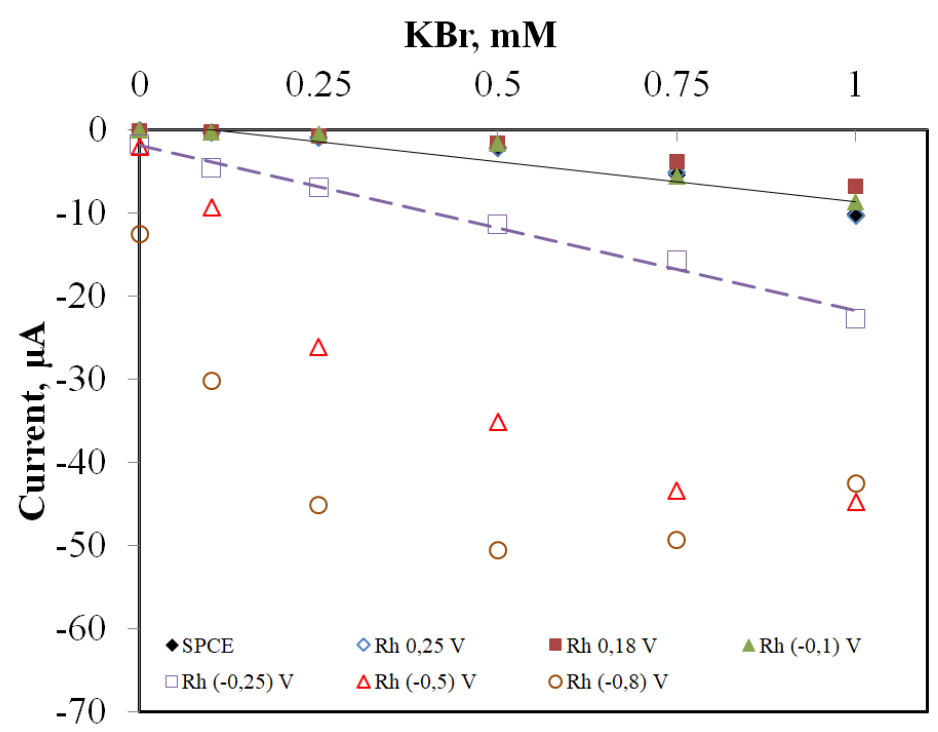

Figure 7.3. Effect of potential for Rh-Nps generation, in cathodic peak at low Br- concentrations. CSV peak currents recorded from 1.11 to - $0.50 \mathrm{~V}$ (anodic stripping of $1.25 \mathrm{~V}$, during $20 \mathrm{~s}$ ) at SPCE for $150 \mu \mathrm{L}$ of 0, 0.10, 0.25, 0.50, 0.75 and $1 \mathrm{mM}$ of $\mathrm{KBr}$ in PBS (0.05 M, $\mathrm{pH} \mathrm{7,} \mathrm{and} \mathrm{0.01} \mathrm{M}$ of $\mathrm{NaCl})$. Scan rate: $0.10 \mathrm{~V} \mathrm{~s}^{-1}$.

Deposition of Rh-Nps at $-0.25 \mathrm{~V}$ yielded a linear slope for the tested bromide concentrations and was selected as the deposition potential for Rh-Nps. The time required for Rh-Nps deposition was also optimized and the SPCE surface was characterized by SEM analysis. The chronoamperometry at $-0.25 \mathrm{~V}$, was performed during $0,30,120$ and $480 \mathrm{~s}$, and the effect on bromide detection was evaluated. In figure 7.4, the SEM image shows rhodium nanostructures on the carbon surface, following an electrodeposition time of 480 s. These data agree with the electrochemical determination of low bromide concentrations, which was only possible when $480 \mathrm{~s}$ for Rh electrodeposition are performed, showing the extent to which, this nano-deposit favored the bromide redox process. Moreover, the comparison of cyclic voltammetric scans performed in the absence and in the presence of Rh-Nps also indicates the surface modification (Figure 7.5). 


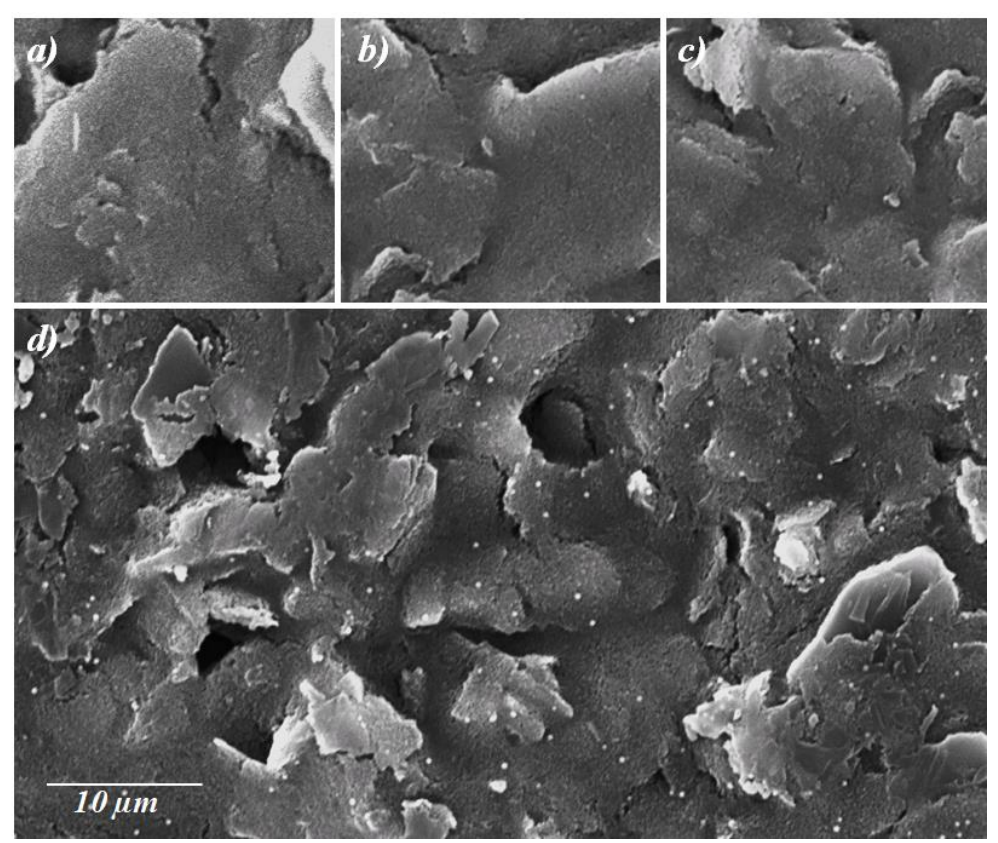

Figure 7.4. Scanning electron micrographs (2.000 x magnification) of the electrode surfaces. RhNps deposit performed onto SPCE by chronoamperometric deposition at $-0.25 \mathrm{~V}$, during a) $0 \mathrm{~s}$, (b) $30 \mathrm{~s}, \mathrm{c)} 120 \mathrm{~s}$, and d) $480 \mathrm{~s}$.

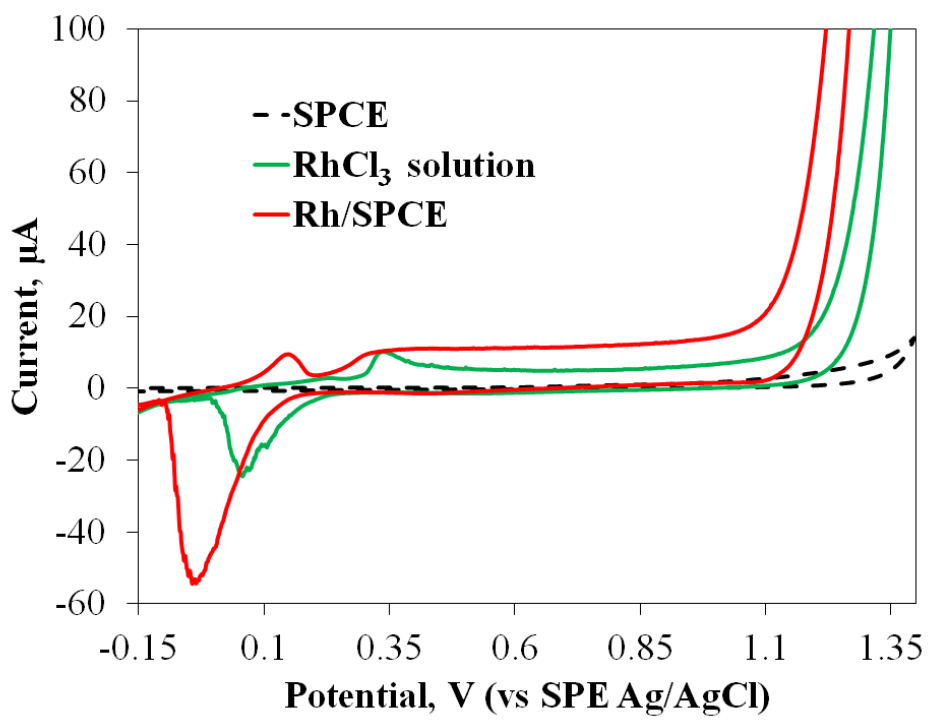

Figure 7.5. Cyclic voltammetric scans obtained in a $0.5 \mathrm{M} \mathrm{H}_{2} \mathrm{SO}_{4}$ solution using SPCE (Black dotted line), $\mathrm{Rh} / \mathrm{SPCE}$ (red line) and in $0.1 \mathrm{mM}$ of $\mathrm{RhCl}_{3}$ in $0.5 \mathrm{M} \mathrm{H}_{2} \mathrm{SO}_{4}$ using SPCE (green line) $\left(\right.$ Scan rate $\left.=0.1 \mathrm{~V} \mathrm{~s}^{-1}\right)$. 


\subsubsection{Chloride influence}

Chloride is a ubiquitous anion, frequently found in samples that also contain bromide. Hence, it was considered of interest to evaluate the effect of chloride in the Rh/SPCE-based sensor performance, as bromide detection can be altered in its presence. The chloride effect in the bromide CSV peaks was checked when using SPCE and Rh/SPCE. Moreover, the anodic stripping effect was verified under those same conditions.

Electrode modification with Rh-Nps proves to be essential in the determination of lower concentrations of bromide. Further, this effect is greatly improved in the presence of chloride, demonstrating that this ubiquitous halide take part in the detection of low concentrations of bromide. When the supporting electrolyte was formulated with different amounts of $\mathrm{NaCl}$, an increase in the signal for bromide concentrations $(<1 \mathrm{mM})$ was observed. Additionally, this increase was more noticeable when the SPCE was modified with Rh-Nps, showing the synergetic effect of both elements (Figure 7.6).

The electrodeposited nanostructures of rhodium and the presence of chloride are both evidencing smaller concentrations of bromide, increasing the sensitivity of the device and improving the method limit of detection. In fact, this could be explained by the oxidative effect of the chlorine, also generated in the primary anodic stage. The fast switch from the anodic step to the cathodic potential sweep of the CSV promotes the reduction of adsorbed bromine, making it possible to obtain reproducible reduction peaks. Similar adsorption behavior was previously described for chlorine gas on a Pt working electrode [32]. Thus, a $0.05 \mathrm{M}$ PBS solution, supplemented with $0.05 \mathrm{M} \mathrm{NaCl}$, was used as the supporting electrolyte, to avoid changes in the bromide peaks obtained in the real sample analysis, which can contain chloride concentrations. 
SPCE
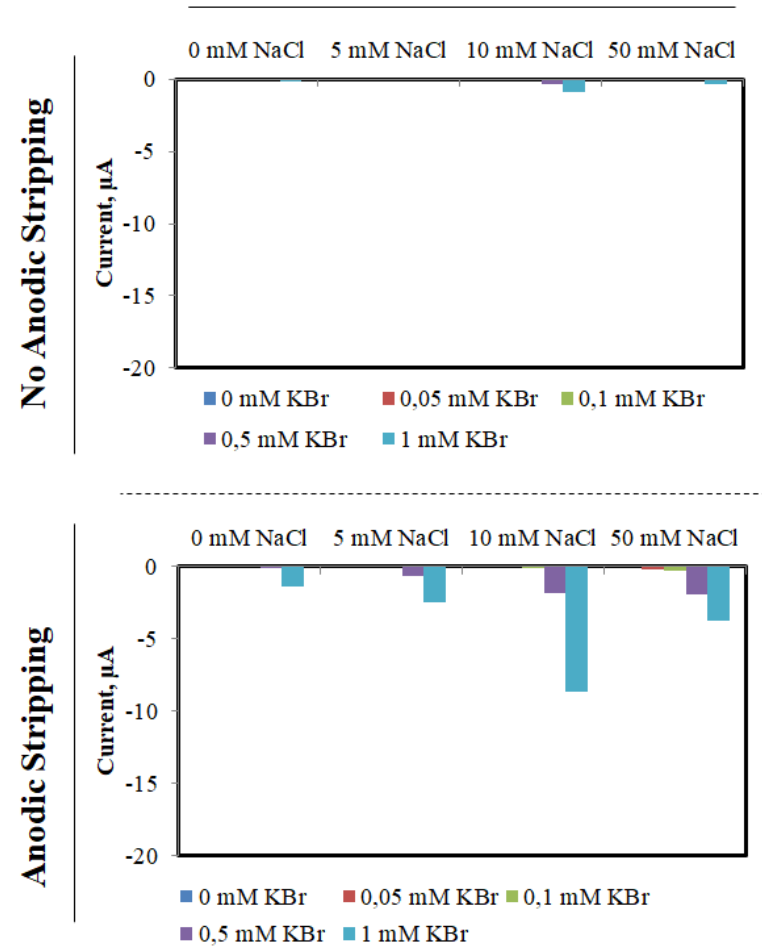

$\mathrm{Rh} / \mathrm{SPCE}$
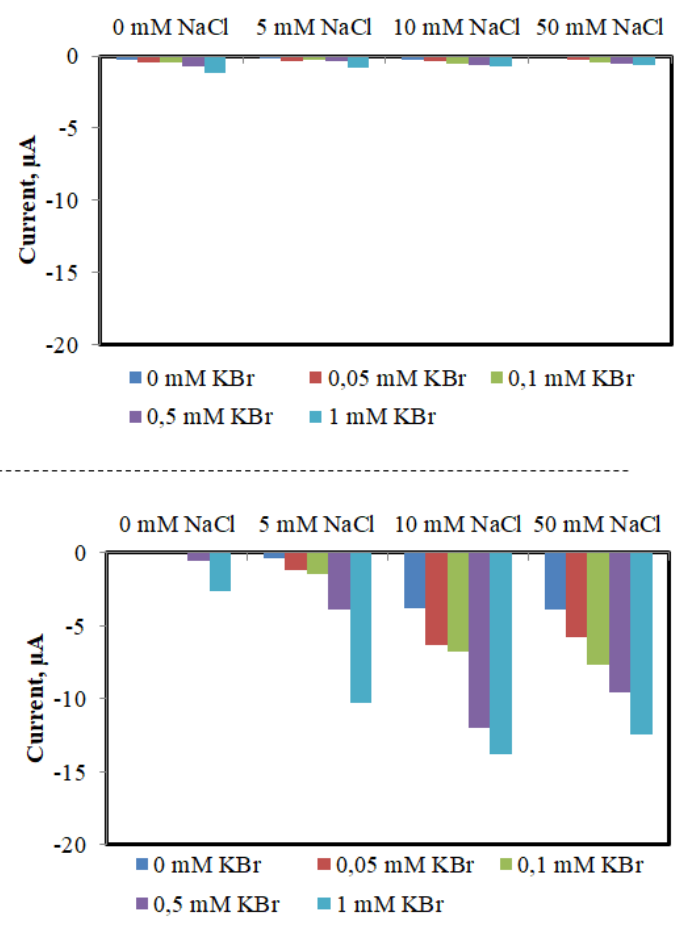

Figure 7.6. Synergic effect of anodic stripping $(+1.25 \mathrm{~V}, 20 \mathrm{~s})$ and chloride, in $\mathrm{Br}^{-}(<1 \mathrm{mM}) \mathrm{CSV}$ peaks, at SPCE and Rh/SPCE. Current intensities recorded in PBS with 0, 5, 10 and $50 \mathrm{mM}$ of $\mathrm{NaCl}(\mathrm{pH} 7)$.

\subsubsection{Characterization of Rh/SPCE-based sensor for bromide determination}

The accuracy of the proposed method was established in terms of reproducibility. Using the optimum voltammetric conditions, and PBS $0.05 \mathrm{M}$ with $0.05 \mathrm{M}$ of $\mathrm{NaCl}$ as supporting electrolyte, several calibration curves for a bromide concentration range from 0 to $40 \mathrm{mM}$ were performed. In table 7.2, the parameters of the calibration curves obtained for five replicas of different $\mathrm{Rh} / \mathrm{SPCE}$ bromide sensors are shown. The sensor reproducibility value, determined in terms of the residual standard deviation (RSD\%) associated with these calibration curves slopes, was $2.58 \%$ (Figure 7.7). A limit of detection (LOD) of $39 \mu \mathrm{M}$ was obtained for the Rh/SPCE-based sensor, using the LOD $=3 S_{x / y} / b$ equation, where, $S_{x / y}$ 
is the estimated standard deviation of the blank, and $b$ is the average slope of the five calibration curves $[33,34]$.

Table 7.2. Calibration parameters obtained through linear regression for the determination of bromide, using five different $R h / S P C E$ based sensor.

\begin{tabular}{ccccccc}
\hline $\boldsymbol{R} h /$ SPCE sensors & Slope $\left(\mu \mathrm{A} \mathbf{m M}^{-1}\right)$ & $\boldsymbol{R}^{2}$ & $\begin{array}{c}\text { Slopes average } \\
\left(\boldsymbol{\mu A} \mathbf{m M}^{-1}\right)\end{array}$ & Deviation & $\boldsymbol{R S D}(\%)$ & $\boldsymbol{L O D}(\boldsymbol{\mu M})$ \\
\hline I & -23.09 & 0.996 & & & \\
II & -23.57 & 0.999 & & & \\
III & -24.08 & 0.998 & -23.18 & 0.60 & 2.58 & 39 \\
IV & -22.33 & 0.992 & & & \\
V & -22.87 & 0.993 & & & \\
\hline
\end{tabular}
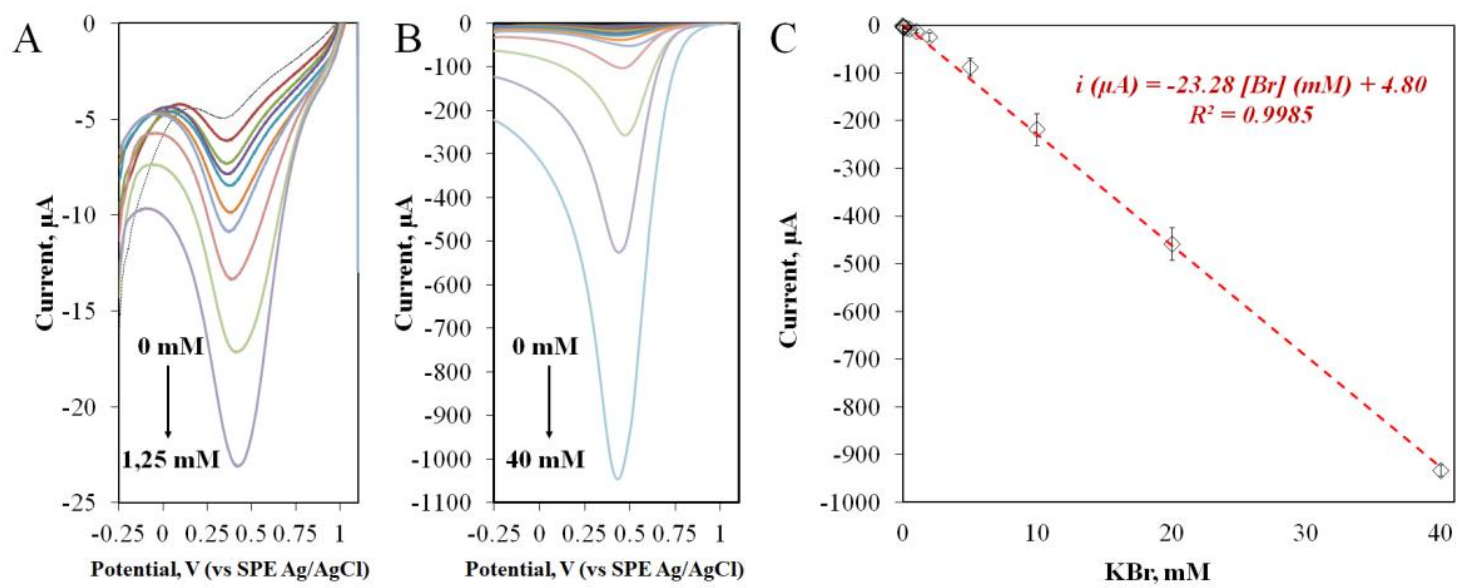

Figure 7.7. Rh/SPCE-based sensor responses obtained under optimal conditions. CSV peaks recorded in a $150 \mu \mathrm{L}$ drop of supporting electrolyte with bromide ranging from (A) 0 to $1.25 \mathrm{mM}$ and (B) from 0 to $40 \mathrm{mM}$. (C) Average calibration curve and associated deviation registered for five different sensors.

Despite the disposable characteristics of the SPEs, repeatability studies were also performed using the same Rh/SPCE-based sensor. Over simultaneous measurements, the 
sensor displays a lack of stability of bromide determination, observable in the variation of the calibration slopes and in their linearity (Figure 7.8). Moreover, these repeated measurements led to peak deformation, increasing the blank signal, which affected the performance of the device when determining lower concentrations of bromide.

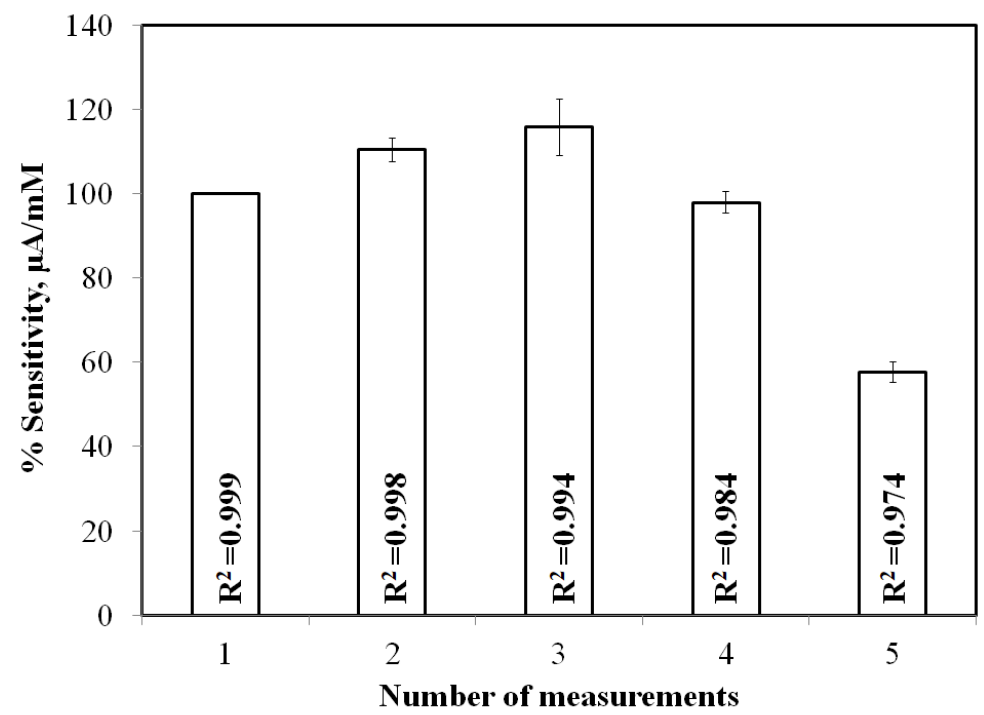

Figure 7.8. Stability of Rh/SPCE sensor during repeated CSV measurements. Results expressed as the average percentage of sensitivity $(\mu \mathrm{A} / \mathrm{mM})$, obtained for three different sensors, and the respective average regression coefficient. The error bars correspond to the standard deviations of the calibration slopes.

\subsubsection{Interferents study}

The device selectivity in detecting bromide anions against the halides, $\mathrm{F}^{-}$and $\mathrm{I}^{-}$, and other anions, such as $\mathrm{NO}_{3}{ }^{-}, \mathrm{CO}_{3}{ }^{2-}$ and $\mathrm{SO}_{4}{ }^{2-}$, was evaluated for $1 \mathrm{mM}$ of bromide (Table 7.3). Chloride, used in excess in the supporting electrolyte, was not considered in the interference study, as the presence of this anion improved bromide detection.

The current variation showed a very low interference effect for $\mathrm{F}^{-}, \mathrm{NO}_{3}{ }^{-}, \mathrm{CO}_{3}{ }^{2-}$ and $\mathrm{SO}_{4}{ }^{2-}$, with small variations in the bromide cathodic peak current, even for a 10-fold excess of the 
interfering anions. In contrast, the $\mathrm{I}^{-}$effect was more noticeable, affecting the cathodic current of $1 \mathrm{mM}$ of bromide. A variation in peak current was noted when the halides, $\mathrm{I}^{-}$and $\mathrm{Br}^{-}$, were both in the same concentration, since $\mathrm{I}^{-}$cathodic peaks starts to rise, hindering the bromide process. The tested $\mathrm{I}^{-}$concentrations of $0.20,0.50$ and $1 \mathrm{mM}$ affected the sensor response for bromide at low concentrations. Even so, the sensor proved its capability to overpass this interference at higher bromide concentrations. Yet, the amount of $\mathrm{I}^{-}$in the samples is often low and interference is negligible. Brines from hydraulic fracturing and

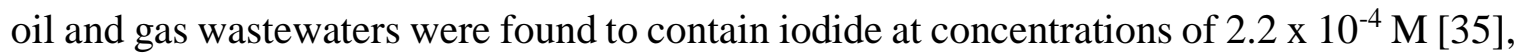
and at even smaller concentrations in seawater $\left(4.7 \times 10^{-7} \mathrm{M}\right)$ [36]. Hence, the selectivity of the $\mathrm{Rh} / \mathrm{SPCE}-$ based sensor is still suitable in real sample analysis, as discussed later.

Table 7.3. Effect of the concentration of interfering species in the bromide peak current percentage $\left(\% I_{p}\right)$.

\begin{tabular}{cccc}
\hline \multirow{2}{*}{ Interference } & \multirow{2}{*}{ [Interference] } & \multicolumn{2}{c}{$\% \boldsymbol{I}_{\boldsymbol{p}}(\boldsymbol{\mu A})$} \\
\cline { 3 - 4 } $\mathrm{F}^{-}$ & $1 \mathrm{mM}$ & 98.71 & - \\
& $10 \mathrm{mM}$ & 97.08 & - \\
& $0.2 \mathrm{mM}$ & 50.34 & 100.01 \\
$\mathrm{I}^{-}$ & $0.5 \mathrm{mM}$ & 75.02 & 99.50 \\
& $1 \mathrm{mM}$ & 146.96 & 98.25 \\
& $1 \mathrm{mM}$ & 99.12 & - \\
$\mathrm{NO}_{3}{ }^{-}$ & $10 \mathrm{mM}$ & 108.85 & - \\
& $1 \mathrm{mM}$ & 102.55 & - \\
$\mathrm{SO}_{4}{ }^{2-}$ & $10 \mathrm{mM}$ & 100.69 & - \\
& $1 \mathrm{mM}$ & 107.40 & - \\
$\mathrm{CO}_{3}{ }^{2-}$ & $10 \mathrm{mM}$ & 98.07 &
\end{tabular}




\subsubsection{Bromide determination in real-life samples}

The effectiveness of the $\mathrm{Rh} / \mathrm{SPCE}-$ based sensor for bromide determination was verified with several real-life samples. The analysis of bromide in seawater is first presented in this study. This matrix is of interest, as the brines from hydraulic fracturing contain higher amounts of dissolved salts than seawater [8]. No bromide content was detected in the raw seawater sample. Thus, this sample was spiked with 2.5 and $5 \mathrm{mM}$ of $\mathrm{KBr}$ and evaluated by the standard addition method. The sensor demonstrated suitable correlations with the added amounts, determining $2.50 \pm 0.08 \mathrm{mM}$ and $5.07 \pm 0.12 \mathrm{mM}$ of bromide, recovering respectively $99.33 \%$ and $100.45 \%$ from the matrix (Figure 7.9).
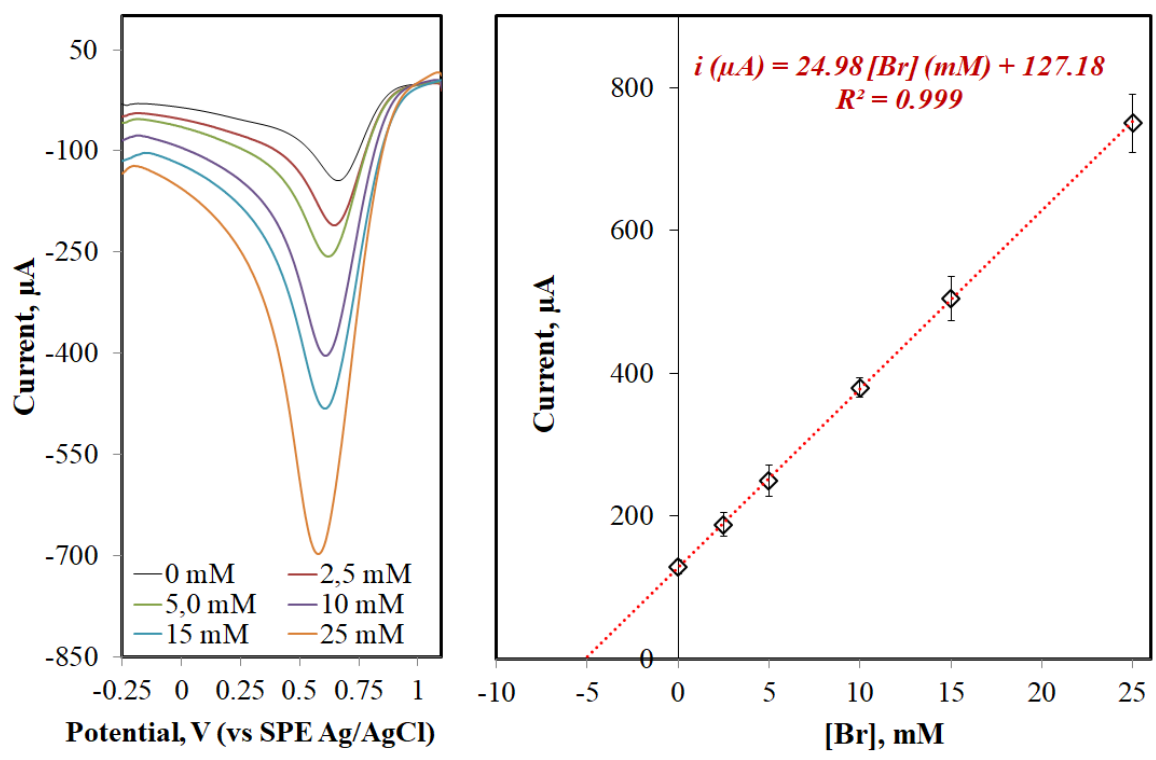

Figure 7.9. Typical voltammograms in single drop analysis of $5 \mathrm{mM}$ bromide spiked in a real seawater sample, by standard addition method ( $n=3)$. (0 mM correspond to $5 \mathrm{mM}$ spiked seawater).

Cetyltrimethylammonium bromide (CTAB), a quaternary ammonium surfactant and a component of a broad-spectrum antiseptic [5], was also analyzed to determine the amount of this halide present in a $6 \mathrm{mM}$ synthetic solution, prepared on the supporting electrolyte. 
The Rh/SPCE-based sensor proved its capability to determine the expected bromide concentration $(5.82 \pm 0.18 \mathrm{mM})$, which can be related to the amount of tensioactive present in the solution.

Table 7.4. Recoveries of bromide in seawater, cetyltrimethylammonium bromide (CTAB) solution, dextromethorphan hydrobromide and ipratropium bromide by standard addition method $(n=3)$.

\begin{tabular}{|c|c|c|c|c|c|c|}
\hline Samples & $\begin{array}{l}\text { Spike Br } \\
\quad(\mathrm{mM})\end{array}$ & $\begin{array}{c}\text { Theoretical } \\
\mathrm{Br}^{-}(\mathrm{mM})\end{array}$ & $\begin{array}{l}\text { Detected Br } \\
\quad(\mathrm{mM})\end{array}$ & $\begin{array}{c}95 \% \text { confidence } \\
\text { interval for mean } \\
(\mathrm{mM}) \\
\end{array}$ & $\begin{array}{c}\text { Recovery } \\
\text { (\%) }\end{array}$ & $\begin{array}{c}R S D \\
(\%)\end{array}$ \\
\hline Seawater & 2.50 & 2.50 & $\begin{array}{l}2.39 \\
2.50 \\
2.56\end{array}$ & $2.50 \pm 0.08$ & $\begin{array}{c}95.56 \\
100.04 \\
102.40\end{array}$ & 2.84 \\
\hline Seawater & 5.00 & 5.00 & $\begin{array}{l}5.07 \\
4.88 \\
5.12\end{array}$ & $5.07 \pm 0.12$ & $\begin{array}{c}101.48 \\
97.50 \\
102.38\end{array}$ & 2.09 \\
\hline CTAB surfactant & - & 6.00 & $\begin{array}{l}5.82 \\
6.13 \\
5.75\end{array}$ & $5.82 \pm 0.18$ & - & 2.80 \\
\hline $\begin{array}{l}\text { Dextromethorphan } \\
\text { hydrobromide }^{\text {a }}\end{array}$ & - & $4.00^{\mathrm{b}}$ & $\begin{array}{l}3.90 \\
3.73 \\
3.80\end{array}$ & $3.80 \pm 0.08$ & - & 1.80 \\
\hline $\begin{array}{l}\text { Ipratropium } \\
\text { bromide }\end{array}$ & - & $0.60^{\mathrm{b}}$ & $\begin{array}{l}0.66 \\
0.63 \\
0.66\end{array}$ & $0.66 \pm 0.02$ & - & 2.11 \\
\hline
\end{tabular}

$\bar{a}$ - Methanolic extraction performed before measurement. ${ }^{b}$ - Labeled bromide concentration of the commercial pharmaceutical formulations. RSD (\%) value associated with the detected $\mathrm{Br}^{-}$concentrations in each sample standard addition method.

The $\mathrm{Rh} / \mathrm{SPCE}$ sensor was also applied to two commercial pharmaceutical formulations, dextromethorphan hydrobromide and ipratropium bromide, detecting $3.80 \pm 0.08 \mathrm{mM}$ and $0.66 \pm 0.02 \mathrm{mM}$, respectively. The concentration determined in these samples closely correlated the bromide value, measured by the standard addition method, with the labeled values of $4.00 \mathrm{mM}$ and $0.60 \mathrm{mM}$ for dextromethorphan hydrobromide and for ipratropium bromide, respectively. 
The results, summarized in table 7.4, demonstrate that the proposed sensor is applicable to real sample analysis, achieving high accuracy, as indicated by the RSD values.

\subsection{CONCLUSIONS}

A simple method, capable of determining bromide in several real-life samples, by single drop analysis, has been developed. The proposed voltammetric Rh/SPCE-based sensor has proven its highly selective, precise and fast capability to determine bromide in different matrices, offering a very short analysis time (33 s for each measurement). Moreover, this disposable device, presents a low limit of detection of $39 \mu \mathrm{M}$, and highly accurate bromide determination $(\mathrm{RSD} \%=2.58)$. The linear range up to $40 \mathrm{mM}$ is ideal for the detection of bromide concentrations that can be found in several types of samples, making this sensor a versatile analytical tool. The real-life samples were analyzed with no pretreatment, except for the dextromethorphan hydrobromide formulation, resulting in an easy-to-use method for the evaluation of bromide in a broad range of samples.

\subsection{REFERENCES}

[1] S.N. Davis, D.O. Whittemore, J. Fabryka-Martin, Uses of chloride/bromide ratios in studies of potable water, Ground Water. 36 (1998) 338-350.

[2] T.A. Ban, Pharmacotherapy of mental illness - A historical analysis, Prog. NeuroPsychopharmacology Biol. Psychiatry. 25 (2001) 709-727.

[3] T. Quast, M. Combs, S. Edwards, Pharmacokinetics of bromide in adult sheep following oral and intravenous administration, Aust. Vet. J. 93 (2015) 20-25. 
[4] B. Gindiciosi, V. Palus, S. Eminaga, E. Villiers, G. Bruto Cherubini, Serum bromide concentrations following loading dose in epileptic dogs, J. Small Anim. Pract. 55 (2014) $108-111$.

[5] E. Ito, K.W. Yip, D. Katz, S.B. Fonseca, D.W. Hedley, S. Chow, G.W. Xu, T.E. Wood, C. Bastianutto, A.D. Schimmer, S.O. Kelley, Potential use of cetrimonium bromide as an apoptosis- promoting anticancer agent for head and neck cancer., Mol. Pharmacol. 76 (2009) 969-983.

[6] M.R. Flórez, M. Resano, Direct determination of bromine in plastic materials by means of solid sampling high-resolution continuum source graphite furnace molecular absorption spectrometry, Spectrochim. Acta - Part B At. Spectrosc. 88 (2013) 32-39.

[7] M. Sun, G. V. Lowry, K.B. Gregory, Selective oxidation of bromide in wastewater brines from hydraulic fracturing, Water Res. 47 (2013) 3723-3731.

[8] K.B. Gregory, R.D. Vidic, D.A. Dzombak, Water management challenges associated with the production of shale gas by hydraulic fracturing, Elements. 7 (2011) $181-186$.

[9] G. Hunter, Bromide Determination in Body Fluids, Biochem J. 54 (1952) 42-45.

[10] F. Zitomer, L.J. L., Spectrophotometric Determination of Bromide Ion in Water, Anal. Chem. 35 (1963) 146-149.

[11] H. Staerk, A. Suess, Bromine Content of Vegetables and its Accumulation After Soil Fumigation with Methyl Bromide, Usind Neuron Activation Analysis, in: Comp. Stud. Food Environ. Contam., 1974: pp. 417-427.

[12] H.F. Dobolyi, Field Determination of Bromide in Water, Anal. Chem. 56 (1984) 2961-2963. 
[13] M.E. Miller, J.M. Cosgriff, G.B. Forbes, Bromide space determination using anionexchange chromatography for measurement of bromide, Am. J. Clin. Nutr. 50 (1989) 168171.

[14] M. Neal, C. Neal, H. Wickham, S. Harman, Determination of bromide, chloride, fluoride, nitrate and sulphate by ion chromatography: Comparisons of methodologies for rainfall, cloud water and river waters at the Plynlimon catchments of mid-Wales, Hydrol. Earth Syst. Sci. 11 (2007) 294-300.

[15] D. Midgley, A Bromide-Selective Electrode-Redox Electrode Cell for the Potentiometric determination of Bromine and Free Residual Chlorine, Talanta. 30 (1983) $547-554$.

[16] A.M. Gerakis, M.A. Koupparis, Studies of Cetyltrimethyl-Ammonium Bromide Micellar System Using a Bromide Selective Electrode, Talanta. 41 (1994) 765-773.

[17] J.F. Van Staden, Flow Injection Determination of Inorganic Bromide in Soils with a Coated Tubular Solid-state Bromide-selective Electrode, Analyst. 112 (1987) 595-599.

[18] B. Bennett, J. Chang, A.J. Bard, Mechanism of the $\mathrm{Br}^{-} / \mathrm{Br}_{2}$ Redox Reaction on Platinum and Glassy Carbon Electrodes in Nitrobenzene by Cyclic Voltammetry, Electrochim. Acta. 219 (2016) 1-9.

[19] S.D. Pretty, A.Y. Musa, J.C. Wren, Reactions of Bromide and Iodide Ions with Silver Oxide Films on Ag Substrates, J. Electrochem. Soc. 160 (2012) H13-H21.

[20] O.D. Renedo, M.A. Alonso-Lomillo, M.J.A. Martínez, Recent developments in the field of screen-printed electrodes and their related applications., Talanta. 73 (2007) 20219.

[21] M.H. Chiu, W.L. Cheng, G. Muthuraman, C.T. Hsu, H.H. Chung, J.M. Zen, A disposable screen-printed silver strip sensor for single drop analysis of halide in biological samples, Biosens. Bioelectron. 24 (2009) 3008-3013. 
[22] J. Bujes-Garrido, D. Izquierdo-Bote, A. Heras, A. Colina, M.J. Arcos-Martínez, Determination of halides using Ag nanoparticles-modified disposable electrodes. A first approach to a wearable sensor for quantification of chloride ions, Anal. Chim. Acta. 1012 (2018) 42-48.

[23] H. Zhai, H. Wang, S. Wang, Z. Chen, S. Wang, Q. Zhou, Y. Pan, Electrochemical determination of mangiferin and icariin based on Au-AgNPs/MWNTs-SGSs modified glassy carbon electrode, Sensors Actuators, B Chem. 255 (2018) 1771-1780.

[24] R. Tan, D. Wu, S. Xu, Y. Zhu, D. Xiong, L. Wang, P. Yang, P.K. Chu, Electrocatalytic hydrogen evolution of palladium nanoparticles electrodeposited on nanographene coated macroporous electrically conductive network, Int. J. Hydrogen Energy. 43 (2018) 2171-2183.

[25] Z. Bo, M. Yuan, S. Mao, X. Chen, J. Yan, K. Cen, Decoration of vertical graphene with tin dioxide nanoparticles for highly sensitive room temperature formaldehyde sensing, Sensors Actuators, B Chem. 256 (2018) 1011-1020.

[26] B. Molinero-Abad, D. Izquierdo, L. Pérez, I. Escudero, M.J. Arcos-Martínez, Comparison of backing materials of screen printed electrochemical sensors for direct determination of the sub-nanomolar concentration of lead in seawater, Talanta. 182 (2018) $549-557$.

[27] M. Barquero-Quirós, M.J. Arcos-Martínez, Effect of nanoparticles on modified screen printed inhibition superoxide dismutase electrodes for aluminum, Sensors. 16 (2016).

[28] V. V. Shumyantseva, S. Carrara, V. Bavastrello, D.J. Riley, T. V. Bulko, K.G. Skryabin, A.I. Archakov, C. Nicolini, Direct electron transfer between cytochrome P450scc and gold nanoparticles on screen-printed rhodium-graphite electrodes, Biosens. Bioelectron. 21 (2005) 217-222. 
[29] V.A. Gatselou, D.L. Giokas, A.G. Vlessidis, M.I. Prodromidis, Rhodium nanoparticle-modified screen-printed graphite electrodes for the determination of hydrogen peroxide in tea extracts in the presence of oxygen, Talanta. 134 (2015) 482-487.

[30] Z. Song, H. Chang, W. Zhu, C. Xu, X. Feng, Rhodium Nanoparticle-mesoporous Silicon Nanowire Nanohybrids for Hydrogen Peroxide Detection with High Selectivity, Sci. Rep. 5 (2015) 1-4.

[31] M. Łukaszewski, H. Siwek, A. Czerwiński, Electrochemical behavior of thin polycrystalline rhodium layers studied by cyclic voltammetry and quartz crystal microbalance, Electrochim. Acta. 52 (2007) 4560-4565.

[32] K. Murugappan, D.W.M. Arrigan, D.S. Silvester, Electrochemical Behavior of Chlorine on Platinum Microdisk and Screen-Printed Electrodes in a Room Temperature Ionic Liquid, J. Phys. Chem. C. 119 (2015) 23572-23579.

[33] J. Mocak, A. M. Bond, S. Mitchell, G. Scollary, A statistical overview of standard (IUPAC and ACS) and new procedures for determining the limits of detection and quantification: Application to voltammetric and stripping techniques (Technical Report), Pure Appl. Chem. 69 (1997) 297-328.

[34] E.R. Ziegel, Statistics and Chemometrics for Analytical Chemistry, 2004.

[35] J.S. Harkness, G.S. Dwyer, N.R. Warner, K.M. Parker, W.A. Mitch, A. Vengosh, Iodide, bromide, and ammonium in hydraulic fracturing and oil and gas wastewaters: Environmental implications, Environ. Sci. Technol. 49 (2015) 1955-1963.

[36] M.A. Kendrick, Halogens in Seawater, Marine Sediments and the Altered Oceanic Lithosphere, in: D.E. Harlov, L. Aranovich (Eds.), Role Halogens Terr. Extraterr. Geochemical Process., Springer International Publishing, Cham, 2018: pp. 591-648. 



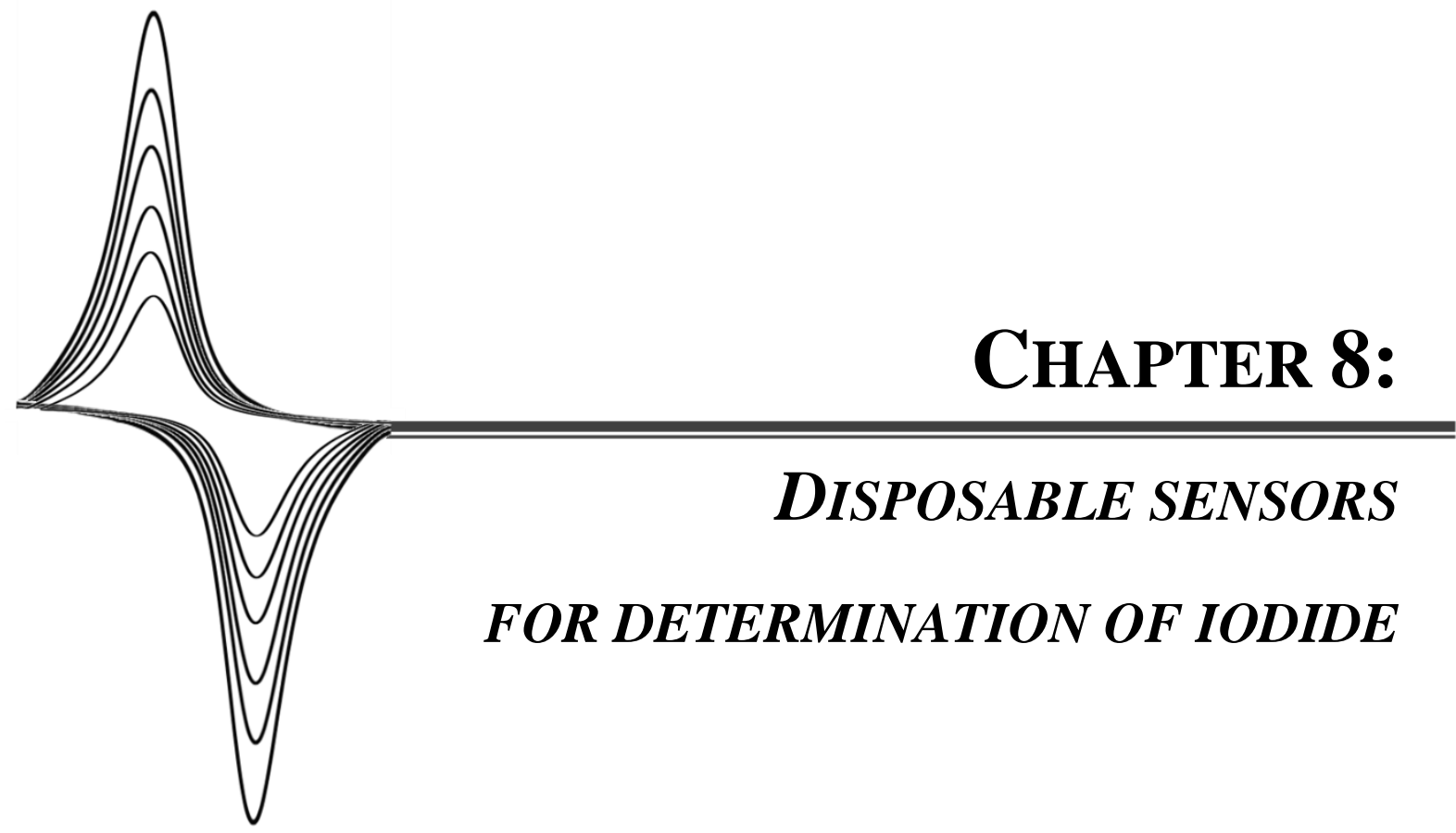



The problematic of iodide deficit over populations have been highlighted by the World Health Organization, which considers iodide deficiency diseases (IDD) to be a public health problem. The main indicator to access IDDs is urinary iodide, since approximately $90 \%$ of the ingested iodide uses this clearance path, with urine being a preferable target for the analysis.

The efficiency of the stripping voltammetric methods applied for halide anions determination, as the shown in the preceding chapters 6 and 7, lead us to approach the problematic of iodide monitoring. For that, in this chapter are proposed two SPCE-based sensors, which required only a single drop of the sample to perform the analysis.

The first approach based on a SPCE proves to selectively determine iodide through the control of the CSV parameters, showing applicability in samples with high content of the anion. The other strategy was fashioned to determine trace iodide concentrations, by merely modified the WE surface with a CS coating. The performance of this new CS/SPCE-based sensor, was compared with the one observed for the SPCE-based sensor, showing improved analytical characteristics and exhibiting a LOD for iodide determination of only $10 \mathrm{nM}$.

The application of both sensors to real-life samples analysis was successfully validated, proving them to be suitable analytical tools for iodide determination in different kinds of samples, including biological matrices.

This work has been published in Talanta 199, 2019, p. 262-269. 



\subsection{INTRODUCTION}

Iodine is an essential element in human nutrition, with recommended daily intake of 150 $\mu \mathrm{g}$ for a regular adult, $250 \mu \mathrm{g}$ for pregnant women and 90 to $120 \mu \mathrm{g}$ for children aged 1 to 13 years [1,2]. Iodide is present in the organism in minimal amounts, mainly in the thyroid gland, where it plays an essential role in the synthesis of thyroid hormones. The synthesis of such hormones is affected when iodide requirements are not met, resulting in iodide deficiency diseases (IDD) such as goiter, hypothyroidism and hyperthyroidism [2-4]. These diseases can emerge across the life-span, from the fetal stage, neonates, children and adults, resulting in symptoms and complications derived from a lack of this micronutrient. Particularly in the case of pregnant women and young children, IDD outcomes are the most devastating, resulting in miscarriage, perinatal mortality and mental retardation [2,3].

The World Health Organization (WHO) considers iodide deficiency to be a public health problem, recommending food iodization with the goal of IDD elimination [3]. Pharmaceutical supplementation, through the use of multivitamin pills, is also widely used $[2,3,5]$. Since approximately $90 \%$ of ingested iodide is excreted in urine, and in the case of IDD a lower fraction is excreted, urinary iodine (UI) is recognized as a suitable marker of dietary intake. According to the WHO guidelines (Table 8.1), iodide content in urine can be lower than $0.32 \mu \mathrm{M}$ and, in some cases, higher than $7.88 \mu \mathrm{M}[2,6]$. Therefore, $\mathrm{UI}$ is a sensitive indicator of changes in iodine consumption across the population and is already used as tool for measuring iodine content [1-3]. Furthermore, radioactive iodine $\left({ }^{131} \mathrm{I}\right)$, intravenous iodinated contrast materials, both used in the treatment and diagnosis of differentiated carcinoma [7-11], and iodide from other pharmaceutical formulations, are excreted via urine, highlighting the significance of this matrix in iodide determination.

Several analytical methods for quantification of iodide are already reported, however only a few are currently used as routine analysis [12]. The most widely used methods are based on the Sandell-Kolthoff reaction (SK), derived from the catalytic effect of iodide on the 
reaction between $\mathrm{Ce}^{4+}$ and $\mathrm{As}^{3+}$ [13]. Methods such as potentiometry using ion-selective electrodes [14,15], atomic absorption spectrometry [16], inductively coupled plasma mass spectrometry [17], optical emission spectrometry [18], neutron activation analysis [19], flow injection analysis [20], gas and liquid chromatographic methods [21,22], UV-visible spectrometry [23], X-ray fluorescence [24], radiotracer analysis by scintillation counting [25], and quartz crystal oscillator [26], are also described for iodide analysis. Most of them require expensive instrumentation and qualified personnel, as well as a complex sample pretreatment prior to analysis. Therefore, these techniques do not meet the needs of developing countries, calling for research into new, inexpensive and reliable methods to perform the analysis [12].

Table 8.1. WHO's urinary iodide range guidelines for assessing iodine nutrition [2,6].

\begin{tabular}{cccc}
\hline Population group & $\begin{array}{c}\text { Median urinary iodine } \\
(\boldsymbol{\mu g} \boldsymbol{L}-\mathbf{1})\end{array}$ & $\begin{array}{c}\text { Corresponding iodide } \\
\text { concentration }(\boldsymbol{\mu M})\end{array}$ & Iodine Status \\
\hline \multirow{2}{*}{ Individuals } & $<20$ & $<0.32$ & Severe deficiency \\
$(\geq 6$ years old $)$ & $50-99$ & $0.32-0.77$ & Moderate deficiency \\
& $100-199$ & $0.79-1.56$ & Mild deficiency \\
& $200-299$ & $1.58-3.14$ & Optimal \\
& $\geq 300$ & $3.15-4.71$ & More than adequate \\
& $<150$ & $\geq 4.73$ & Excessive \\
\hline \multirow{2}{*}{ Pregnant women } & $150-249$ & $<2.36$ & Insufficient \\
& $250-499$ & $2.36-3.92$ & Adequate \\
& $\geq 500$ & $3.94-7.86$ & Above requirements \\
& & $\geq 7.88$ & Excessive \\
\hline
\end{tabular}

In light of this, electrochemical sensors may be the most suitable platforms for iodide determination. Several modifications of such probes were performed to improve the determination in numerous matrices (Table 8.2). Conventional electrode systems based on glassy carbon electrodes [27-34], carbon paste electrodes [27,35], carbon electrode generated by electrospinning [36], gold electrodes [37-39], platinum electrodes [40-43] 
and also fluorine-doped tin oxide electrodes [42], are discussed in the literature. Examples of silver working electrodes [6,44-48] are more frequently found, however these devices are usually limited to a single measurement, since AgI deposits result in electrode sensitivity loss, requiring cleaning steps between each measurement. Conventional electrodes are unpractical for point-of-care use, requiring exhaustive modifications and steps to achieve a satisfactory determination of iodide.

Screen-printed electrodes (SPE), overcome the limitations of conventional electrochemical systems, offering portability, low-cost, disposability and an easy single-drop analysis [49]. SPAgE or modified Ag/SPE [50,51], SPAuE and SPPtE [52], SPCE modified with copper (II)-sulphamethazine Schiff base complex [35] and SPCE modified with an ionic liquid interface $[53,54]$ are described in the literature for iodide determination, however some improvements are needed. The need for complicated electrode modifications, and in some cases a complex sample pretreatment, once again limit their use as point-of-care methods. In fact, none have been successfully used to determine iodide content in human urine (Table 8.2).

SPE modifications based on chitosan (CS), a natural polysaccharide [55], have been shown to improve several sensing platforms [34,39,64-66,56-63], with several works taking advantage of the excellent membrane-forming ability, high permeability to water, good adhesion, high mechanical strength and susceptibility to chemical modifications [64-66]. CS stood out as a suitable agent in electrochemical iodide determination, since the presence of cationic amino groups facilitates the adsorption of this anion on the electrode interfaces $[34,39,67,68]$.

This work presents two disposable, inexpensive and easy-to-use sensors for a quick pointof-care method for iodide determination. One of the proposed sensors was modified with a CS coating, improving the analytical performance to determine trace iodide concentrations. The anodic pretreatment of the sample and the subsequent cathodic sweep to form iodide results in a cathodic voltammetric signal, used to quantify the content of this anion in several real-life samples. 
Table 8.2. Comparison of analytical characteristics of several electrochemical sensing platforms for iodide determination.

\begin{tabular}{|c|c|c|c|c|c|c|c|}
\hline Electrodes & Techniques & $\begin{array}{c}L O D \\
(M)\end{array}$ & $\begin{array}{l}\text { Linear Range } \\
(M)\end{array}$ & $R^{2}$ & $\begin{array}{c}R S D \\
(\%)\end{array}$ & Applications & References \\
\hline SPCE, SPAuE, SPPtE & $\mathrm{AD}$ & NR & $1.0 \times 10^{-4}-3.0 \times 10^{-3}$ & $\begin{array}{c}0.988- \\
0.998\end{array}$ & $4.19-5.26$ & $\begin{array}{c}\text { Tap and agriculture drain } \\
\text { waters }\end{array}$ & [52] \\
\hline AgNps/SPCE & LSV & $5.0 \times 10^{-6}$ & $5.0 \times 10^{-6}-0.8 \times 10^{-4}$ & 0.991 & 3.23 & NR & [51] \\
\hline PEDOT/GCE & $\mathrm{CV}$ & NR & $1.0 \times 10^{-3}-9.0 \times 10^{-3}$ & 0.999 & NR & NR & [31] \\
\hline SPAgE & LSV & $3.1 \times 10^{-6}$ & $10 \times 10^{-3}-20 \times 10^{-3}$ & 0.998 & 5.00 & Pond water & [50] \\
\hline MCNF & DPV & $1.4 \times 10^{-6}$ & $5.0 \times 10^{-6}-0.7 \times 10^{-3}$ & 0.998 & NR & Synthetic urine & [36] \\
\hline Silver-foil & $\mathrm{CV}$ & $1.0 \times 10^{-4}$ & $1.0 \times 10^{-4}-1.0 \times 10^{-1}$ & NR & 3.50 & Food supplement Tablet & [47] \\
\hline CoIIIsalophen/CP & $\mathrm{AD}$ & $4.0 \times 10^{-6}$ & $8.0 \times 10^{-6}-32 \times 10^{-6}$ & 0.998 & 7.00 & $\begin{array}{l}\text { Produced water from an oil } \\
\text { well }\end{array}$ & [27] \\
\hline VAgAmlg microwire & CSV & $0.7 \times 10^{-9}$ & $0.7 \times 10^{-9}-1.0 \times 10^{-7}$ & 0.999 & 5.30 & Estuary river waters & [48] \\
\hline CTA/Co-MnO2/GCE & $\mathrm{AD}$ & $6.6 \times 10^{-9}$ & $2.0 \times 10^{-6}-20 \times 10^{-6}$ & 0.999 & 2.90 & NR & [29] \\
\hline $\begin{array}{c}\mathrm{Cu}(\mathrm{II}) \mathrm{SBS} / \mathrm{CP} \\
\mathrm{Cu}(\mathrm{II}) \mathrm{SBS} / \mathrm{SPCE}\end{array}$ & $\mathrm{PD}$ & $\begin{array}{l}1.0 \times 10^{-5} \\
1.0 \times 10^{-5}\end{array}$ & $\begin{array}{l}1.0 \times 10^{-5}-1.0 \times 10^{-1} \\
1.0 \times 10^{-5}-1.0 \times 10^{-1}\end{array}$ & $\begin{array}{l}\mathrm{NR} \\
\mathrm{NR}\end{array}$ & $\begin{array}{l}\text { NR } \\
\text { NR }\end{array}$ & Seawater & [35] \\
\hline$[(\mathrm{C} 16) 2 \mathrm{Im}] \mathrm{I} / \mathrm{SPE}$ & PD & $8.0 \times 10^{-6}$ & $5.0 \times 10^{-5}-1.0 \times 10^{-1}$ & NR & 0.94 & Iodine containing drugs & [53] \\
\hline $\begin{array}{c}\text { DDMImPF6/Co(TBPC)I/ } \\
\text { SPCE }\end{array}$ & PD & $7.3 \times 10^{-6}$ & $1.0 \times 10^{-5}-1.0 \times 10^{-1}$ & NR & NR & Iodine containing drugs & [54] \\
\hline$(\mathrm{VO}(\mathrm{OC} 3 \mathrm{H} 7) 3) / \mathrm{PPC} / \mathrm{GCE}$ & $\mathrm{AD}$ & $1.0 \times 10^{-7}$ & $5.0 \times 10^{-7}-10 \times 10^{-3}$ & 0.999 & 3.20 & Dry edible seaweed & [32] \\
\hline
\end{tabular}


Table 8.2. Comparison of analytical characteristics of several electrochemical sensing platforms for iodide determination (continuation).

\begin{tabular}{|c|c|c|c|c|c|c|c|}
\hline Electrodes & Techniques & $\begin{array}{c}L O D \\
(M)\end{array}$ & $\begin{array}{c}\text { Linear Range } \\
\text { (M) }\end{array}$ & $R^{2}$ & $\begin{array}{c}R S D \\
(\%)\end{array}$ & Applications & References \\
\hline $\mathrm{AgNps} / \mathrm{GCE}$ & $\mathrm{CV}$ & NR & $0.3 \times 10^{-6}-6.0 \times 10^{-6}$ & 0.999 & NR & Synthetic urine & {$[6]$} \\
\hline $\mathrm{CS} / \mathrm{GCE}$ & ASV & $3.9 \times 10^{-7}$ & $7.9 \times 10^{-7}-5.9 \times 10^{-6}$ & 0.995 & 6.10 & Cydiodine Tablets & [34] \\
\hline $\mathrm{CPB} / \mathrm{GCE}$ & CSV & $8.0 \times 10^{-9}$ & $3.0 \times 10^{-8}-2.0 \times 10^{-5}$ & $\begin{array}{l}0.995- \\
0.998\end{array}$ & 3.28 & $\begin{array}{c}\text { Table salt, pharmaceuticals } \\
\text { and Kelp }\end{array}$ & [28] \\
\hline AuNps - CS/AuE & LSV & $1.2 \times 10^{-7}$ & $3.3 \times 10^{-7}-1.6 \times 10^{-2}$ & 0.999 & 3.30 & Dietary supplement & {$[39]$} \\
\hline SPCE & CSV & $2.9 \times 10^{-6}$ & $5.0 \times 10^{-6}-7.5 \times 10^{-3}$ & 0.996 & 2.86 & Human urine, tap water and & This Work \\
\hline CS/SPCE & CSV & $1.0 \times 10^{-8}$ & $1.5 \times 10^{-7}-5.0 \times 10^{-4}$ & 0.998 & 2.71 & pharmaceuticals & \\
\hline
\end{tabular}

[(C $\left.\left.\mathrm{C}_{16}\right)_{2} \mathrm{Im}\right] \mathrm{I}$ - 1,3-dihexadecylimidazolium iodide; AD - Amperometric detection; AgNps - Silver nanoparticles; ASV - Anodic stripping voltammetry; AuNps - Gold nanoparticles; CP - Carbon paste; CS - Chitosan; CSV - Cathodic stripping voltammetry; CV- Cyclic voltammetry; CTA - Cetyltrimethylammonium; Co- $\mathrm{MnO}_{2}$ - Organo-Manganese Dioxide Framework-Doped with Cobalt; Co(TBPC)I - Tetrakis(tert-butyl)phthalocyaninatocobalt- (III) iodide 3; CPB Cetylpyridine bromide; Cu(II)SBS - Copper (II)- sulphamethazine Schiff Base Complex; DDMImPF6 - 1-dodecyl-3-methylimi-dazolium hexafluorophosphate; DPV - Differential pulse voltammetry; GCE - Glassy carbon electrode; LSV - Linear sweep voltammetry; LMIS - Low-melting ionic solids; MCNF Mechanically treated carbon nanofiber; NR - Not reported; PD - Potentiometric detection; PEDOT - poly(3,4-ethylenedioxythiophene); PPC - Poly(propylene carbonate); SPAgE - Screen-printed silver electrode; SPAuE - Screen-printed gold electrode, SPCE - Screen-printed carbon electrode; SPPtE - Screen-printed platinum electrode; $\left(\mathrm{VO}\left(\mathrm{OC}_{3} \mathrm{H}_{7}\right)_{3}\right)$ - Vanadium tri(isoproxide) oxide; VAgAmlg microwire - Vibrating silver amalgam microwire. 


\subsection{EXPERIMENTAL SECTION}

\subsubsection{Chemicals and reagents}

Potassium iodide $(\mathrm{KI})$, potassium chloride, $\mathrm{D}(+)$-Glucose, chitosan and activated charcoal were purchased from Sigma-Aldrich (Steinheim, Germany). Potassium bromide, sodium fluoride, sodium phosphate dibasic dihydrate, sodium phosphate monobasic dihydrate, sodium chloride, sulfuric acid, urea, ammonia solution (25\%), ceric ammonium nitrate and arsenic trioxide were obtained from Merck (Darmstadt, Germany). Ascorbic acid, sodium hydroxide, acetic acid and hydrochloric acid were purchased from Panreac (Barcelona, Spain). Creatinine was obtained from Acros Organics (Geel, Belgium), and Uric Acid from Alfa Aesar.

The urine samples were obtained from volunteers and stored at $-20^{\circ} \mathrm{C}$ until analysis. Urine samples with low and normal iodide content were obtained from an individual with an iodide deficit and from a healthy subject, respectively. Additionally, four urine samples were also obtained from a patient submitted to intravenous iodinated contrast for a radiological scanner, and collected 2, 15, 17 and 20 hours after administration.

Tap water was obtained from our laboratory facilities in University of Burgos (Spain) and analyzed immediately after collection.

The two commercial pharmaceutical formulations, Yodocefol ${ }^{\circledR}$ and Supradyn ${ }^{\circledR}$, were obtained from a local pharmacy. Both pills were dissolved separately in an adequate volume of supporting electrolyte prior to the electrochemical and spectrophotometric analyses.

All reagents were of analytical grade and all solutions were prepared with Milli-Q water (Millipore, Bedford, USA). 
The inks used for the fabrication of SPCE, Electrodag PF-407 A (carbon ink), Electrodag 6037 SS (silver/silver chloride ink), Electrodag 418 (silver ink) and Electrodag 452 SS (dielectric ink), were supplied by Achenson Colloiden (Scheemda, Netherlands). Polyester films (PET) of $0.5 \mathrm{~mm}$ thickness (HIFI Industrial Film, Dardily, France), were used as the printing substrates.

\subsubsection{Apparatus}

A DEK 248 screen-printing system (DEK, Weymouth, UK) was used to fabricate SPEs, using polyester screens with the appropriate stencil. SPEs, were produced following a previously described procedure [69]. A sequential deposition of the appropriate inks defines, the conductive silver tracks, the $\mathrm{Ag} / \mathrm{AgCl}$ pseudo-reference electrode, the carbon counter and working electrodes $\left(0.126 \mathrm{~cm}^{2}\right)$ and the insulating layer.

The solutions $\mathrm{pH}$ was measured with a Crison micro pH 2002, coupled to crison $5200 \mathrm{pH}$ electrode (Crison instruments, Barcelona).

Centrifugation of the samples was carried out in Eppendorf centrifuge 5804.

Electrochemical measurements were performed with a $\mu$ Stat 200 bipontentiostat from Dropsens (Oviedo, Spain), using the Drop-View software package to control the instrument, register and perform the analysis of results.

Spectrophotometric measurements were carried out using a GENESYS ${ }^{\mathrm{TM}} 30$ Visible Spectrophotometer from Thermo Fisher scientific (Massachusetts, USA). The experiments were carried out into a standard (macro) cell with lid, $10 \mathrm{~mm}$, optical glass, from Labbox Labware SL (Barcelona, Spain).

\subsubsection{Electrochemical measurements}

Voltammetric measurements were performed using a phosphate buffer saline $(50 \mathrm{mM}, \mathrm{pH}$ 6.5) supplemented with $10 \mathrm{mM}$ of $\mathrm{NaCl}(\mathrm{PBS})$ as the supporting electrolyte. 
Cyclic voltammetric (CV) experiments for the electrochemical screening of iodide were performed, by drop-casting $150 \mu \mathrm{L}$ of PBS, containing $0,0.5,1,2,4,8$ and $10 \mathrm{mM}$ of $\mathrm{KI}$, onto the electrodic systems, and voltammograms were recorded between 0.00 and +1.25 $\mathrm{V}(\mathrm{vs} \mathrm{SPE} \mathrm{Ag} / \mathrm{AgCl})$ at $0.10 \mathrm{~V} \mathrm{~s}^{-1}$.

For the cathodic stripping voltammetry (CSV) experiments, $150 \mu \mathrm{L}$ of problem solution was drop-cast onto the working area of the sensor, and an anodic deposition step at +0.50 $\mathrm{V}$ (vs SPE $\mathrm{Ag} / \mathrm{AgCl}$ ) was applied for 60 seconds. Immediately after (equilibration time = $0 \mathrm{~s}$ ), a linear sweep voltammetric (LSV) scan was performed from +0.90 to $-0.45 \mathrm{~V}$ at a scan rate of $0.10 \mathrm{~V} \mathrm{~s}^{-1}$, yielding a reduction peak at $+0.05 \mathrm{~V}$ (vs SPE $\mathrm{Ag} / \mathrm{AgCl}$ ). The sensor was rinsed with Milli-Q water prior to subsequent measurements.

\subsubsection{SK spectrophotometric measurements}

When applying the Sandell-Kolthoff (SK) spectrophotometric method, a sample pretreatment was required. Briefly, $0.15 \mathrm{~g}$ of activated charcoal and $5 \mathrm{~mL}$ of the sample were mixed in vortex for $30 \mathrm{~s}$. After that, the mixture was centrifuged at $2500 \mathrm{rpm}$ for 5 minutes. The supernatant was then filtered through a cellulose membrane $(0.45 \mu \mathrm{m})$ and stored in a dark vessel until measurement.

The spectrophotometric measure began with the addition of $750 \mu \mathrm{L}$ of water into the spectrophotometric cell. Subsequently, $125 \mu \mathrm{L}$ of $\mathrm{H}_{2} \mathrm{SO}_{4}: \mathrm{HCl}(0.35 \mathrm{M}: 0.065 \mathrm{M})$ and 125 $\mu \mathrm{L}$ of As (III) (60 mM in water) were added in that order. Afterwards, $125 \mu \mathrm{L}$ of Ce (IV) ( $5 \mathrm{mM}$ in $5 \%$ of $\mathrm{H}_{2} \mathrm{SO}_{4}$ ) was added to the cell and left to react for $90 \mathrm{~s}$. Lastly, $125 \mu \mathrm{L}$ of the sample was added to the mixture and left to react for $30 \mathrm{~s}$, and the decrease of the intensity at $370 \mathrm{~nm}$ was registered after one minute. 


\subsection{RESULTS AND DISCUSSION}

\subsubsection{Electrochemical behavior of iodide}

Cyclic voltammograms of KI solutions recorded using a SPCE exhibit two anodic oxidative peaks, $\mathrm{I}\left(\mathrm{E}_{\mathrm{pa}} \mathrm{I}=+0.58 \mathrm{~V}\right)$ and II $\left(\mathrm{E}_{\mathrm{pa} \mathrm{II}}=+0.90 \mathrm{~V}\right)$, coupled with two corresponding cathodic peaks, IV $\left(\mathrm{E}_{\mathrm{pc} \mathrm{IV}}=+0.22 \mathrm{~V}\right)$ and III $\left(\mathrm{E}_{\mathrm{pc} \mathrm{III}}=+0.62 \mathrm{~V}\right)($ Figure 8.1). The coupled peaks indicate the presence of two quasi-reversible electron transfer steps. The first step produces the couple $\mathrm{I} / \mathrm{IV}$, resulting from the redox reaction between iodide $\left(\mathrm{I}^{-}\right)$and triiodide $\left(\mathrm{I}_{3}^{-}\right)$ions. This process is followed by the redox couple II/III, derived from the redox of both $\mathrm{I}^{-}$and $\mathrm{I}_{3}{ }^{-}$to iodine $\left(\mathrm{I}_{2}\right)$ [36]. Some authors associate this second redox couple (II/III) with the interaction between the generated iodine $\mathrm{I}_{2}$ and the solvent [33]. The current intensity of both redox couple peaks, I/IV and II/III, are increased for higher concentrations of KI and are attributed to the equations. (8.1) and (8.2), respectively.

$$
\begin{aligned}
& 3 I^{-} \underset{\leftarrow}{\leftarrow} I_{3}^{-}+2 e^{-} \\
& 2 I_{3}^{-} \stackrel{\rightarrow}{\leftarrow} 3 I_{2}+2 e^{-}
\end{aligned}
$$

Regarding the intensities obtained in both processes, the couple I/IV results in higher current output, indicating a more sensitive signal for iodide determination. Moreover, the process that produces the cathodic peak IV exhibits a stable potential $\left(\mathrm{E}_{\mathrm{pc}} \mathrm{IV}=+0.22 \mathrm{~V}\right)$, contrary to what occurs with the remaining peaks, that show a potential shift when iodide concentrations are increased.

Accordingly, the cathodic peak IV, which results from triiodide reduction to iodide anions, was selected for the determination of the halide using the SPCE as a sensing platform. 


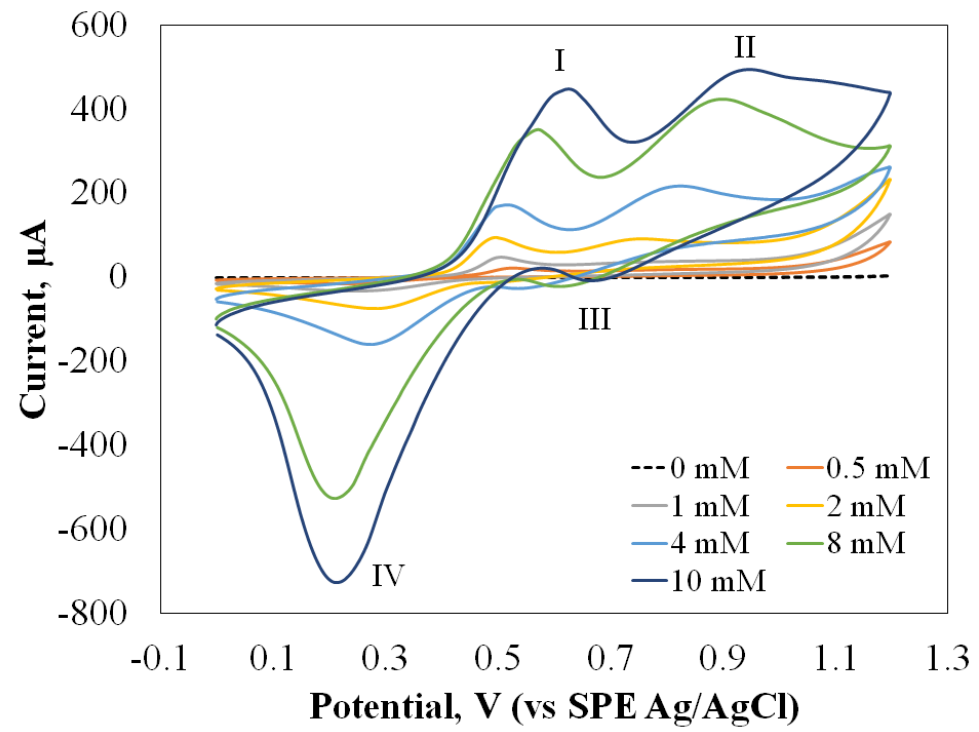

Figure 8.1. Cyclic voltammograms of $0,0.5,1,2,4,8$ and $10 \mathrm{mM} \mathrm{KI}$ in PBS $(\mathrm{pH}=6.5)$ at SPCEbased sensor. Scan rate: $0.10 \mathrm{~V} \mathrm{~s}^{-1}$.

\subsubsection{CSV for a selective determination of iodide}

The variation of peak IV current with iodide concentration was examined using cathodic stripping voltammetry (CSV) and the method was optimized to achieve an improved analysis of this anion. To promote the formation of iodine or triiodide in the sample volume, aiming to increase the voltammetric peak (IV) current, several anodic deposition potentials $\left(E_{d e p}\right)$ were applied at different times $\left(t_{d e p}\right)$, prior to the LSV scan (Figure 8.2). From the experiments performed, a $E_{d e p}+0.50 \mathrm{~V}$ and $t_{d e p} 60 \mathrm{~s}$ were found to be suitable values. For $E_{\text {dep }}$ higher than $+1.25 \mathrm{~V}$, a decrease and a higher variability in the peak current were observed. This may be due to the iodine deposits that occur under these conditions, limiting the electron transfer and the availability of oxidized species.

The selected parameters of $E_{d e p}+0.50 \mathrm{~V}$ and $t_{d e p} 60 \mathrm{~s}$ improved iodide determination, increasing the cathodic peak current. Furthermore, a shift from $0.22 \mathrm{~V}$ to $0.05 \mathrm{~V}$ in the peak potential was observed, reflecting a more favorable process. Moreover, the $E_{d e p}+0.50 \mathrm{~V}$ is in accordance with the potential for the respective anodic quasi-reversible process (Figure 8.1, as peak I), promoting a selective oxidation that gives rise to the posterior 
cathodic potential sweep, the interest peak IV. Lastly, the effect of this anodic step in the analytical performance of the SPCE-based sensor was checked for an iodide range from $5.0 \mu \mathrm{M}$ to $50 \mu \mathrm{M}$ (Figure 8.3 ).
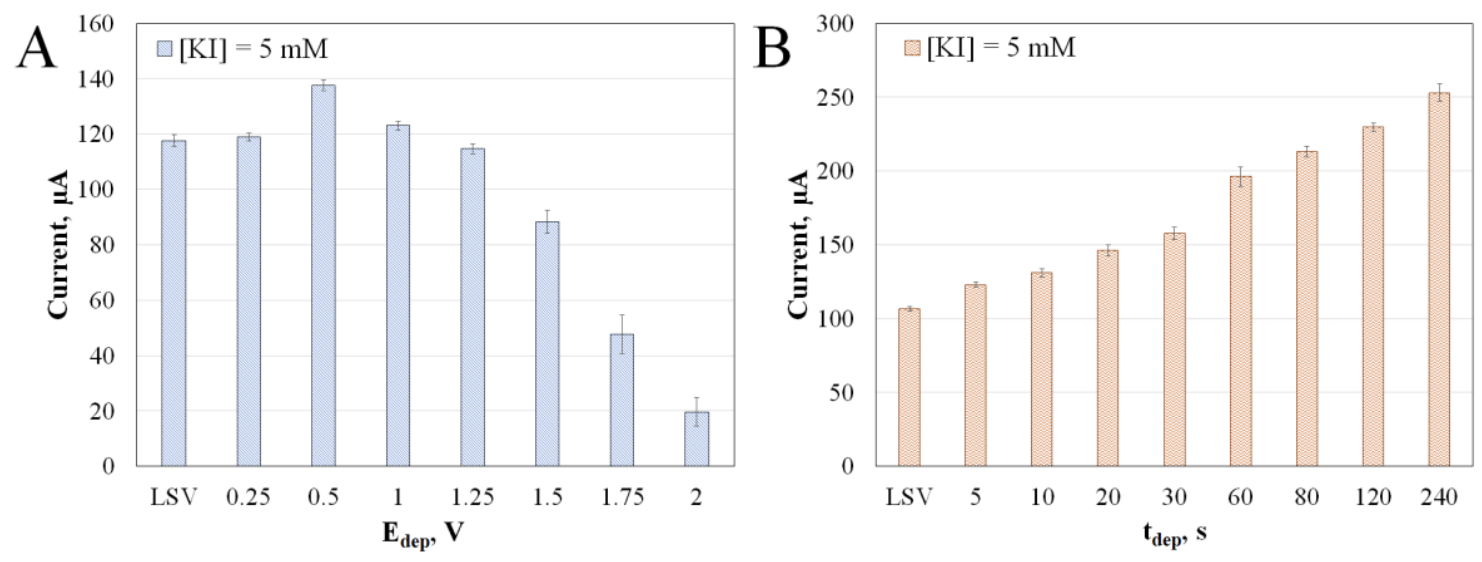

Figure 8.2. CSV setup. Current output recorded at SPCE for $150 \mu \mathrm{L}$ of $5 \mathrm{mM}$ of KI in PBS (pH =6.5) for: (A) Different anodic potentials $\left(E_{\text {dep }}\right)$ applied for $10 \mathrm{~s}$; and $(B) E_{\text {dep }}$ of $0.50 \mathrm{~V}$, applied during different $t_{\text {dep. }}$ Scan rate: $0.10 \mathrm{~V} \mathrm{~s}^{-1}$. Error bars of associated deviation $(n=3)$.

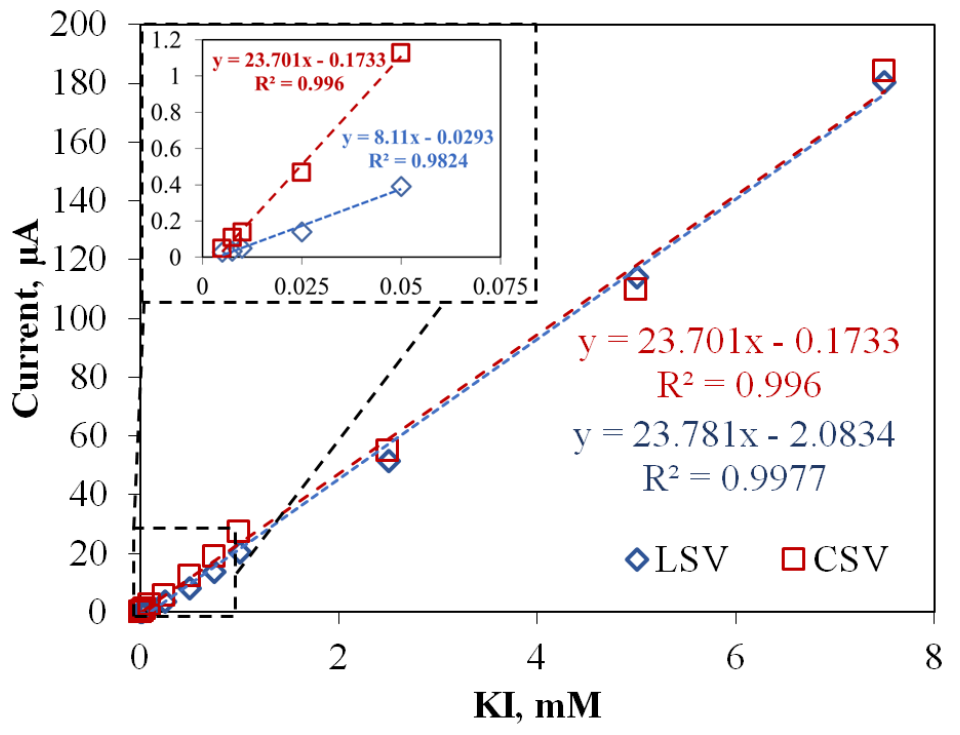

Figure 8.3. Effect of anodic step in CSV determination of iodide. Linearity of potassium iodide concentrations over the analysis range of $5 \mu M$ to $7.5 \mathrm{mM}$, for cathodic LSV and CSV. Inset: $5 \mu M$ to $50 \mu M$. 


\subsubsection{Characterization of the SPCE-based sensor}

The accuracy of the developed method was calculated in terms of reproducibility and repeatability. Under the selected CSV conditions, three calibration curves were performed using different SPCE-based sensors for an iodide concentration range from 0.0 to $7.5 \mathrm{mM}$.

The sensor displays a linear analysis range from $5.0 \mu \mathrm{M}$ to $7.5 \mathrm{mM}$ (Figure 8.4 ), which was used to calculate the sensor's analytical parameters. The reproducibility, determined in terms of the residual standard deviation (RSD\%), associated with the slopes of these calibration curves $\left(23.68 \mu \mathrm{A} \mathrm{mM}^{-1}\right)$ was $3.32 \%$. Using the equation 8.3 , a limit of detection (LOD) of $2.9 \times 10^{-6} \mathrm{M}$, was obtained for the SPCE-based iodide sensor.

$$
\operatorname{LOD}=\frac{3 S_{x / y}}{b}
$$

where, $S_{x / y}$ is the estimated standard deviation of the smallest concentration detected (since the blank has no signal, $S_{x / y}$ for $5.0 \mu \mathrm{M}$ ), and $b$ is the average slope of the three calibration curves $[70,71]$.

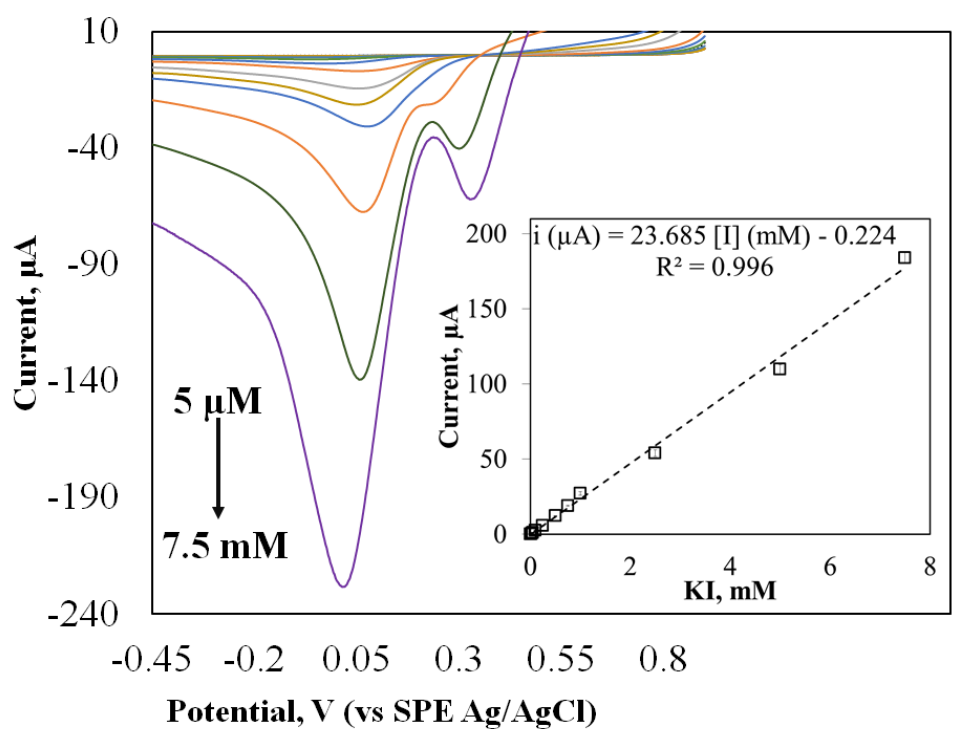

Figure 8.4. CSV peaks recorded at SPCE-based sensor of a $150 \mu \mathrm{L}$ drop of supporting electrolyte containing KI ranging from $5 \mu \mathrm{M}$ to $7.5 \mathrm{mM}$. Inset: Average calibration curve and associated deviation $(n=3)$. 
The sensor repeatability was also checked, demonstrating the stability of each SPCE over a calibration curve. However, the sensor was considered as a disposable device, since after the first calibration assay (15 concentration measurements), an increase of the blank signal was observed (Figure 8.5). This resulted in a cathodic peak corresponding to the iodine accumulated on the working electrode, prohibiting its use for more than one calibration.

Table 8.3. summarizes the analytical parameters obtained for the SPCE-based sensor. The use of this device is limited to matrices with relatively high anion content, rendering it impractical for the determination of trace iodide. Therefore, this sensor is not feasible for analyzing urine form healthy individuals, since the expected iodide concentration in this matrix lies between a range of $0.32 \mu \mathrm{M}$ and $8.0 \mu \mathrm{M}$. With this in mind, the SPCE-based sensor was modified with a chitosan (CS) coating to improve the limit of detection and the sensitivity of the device.

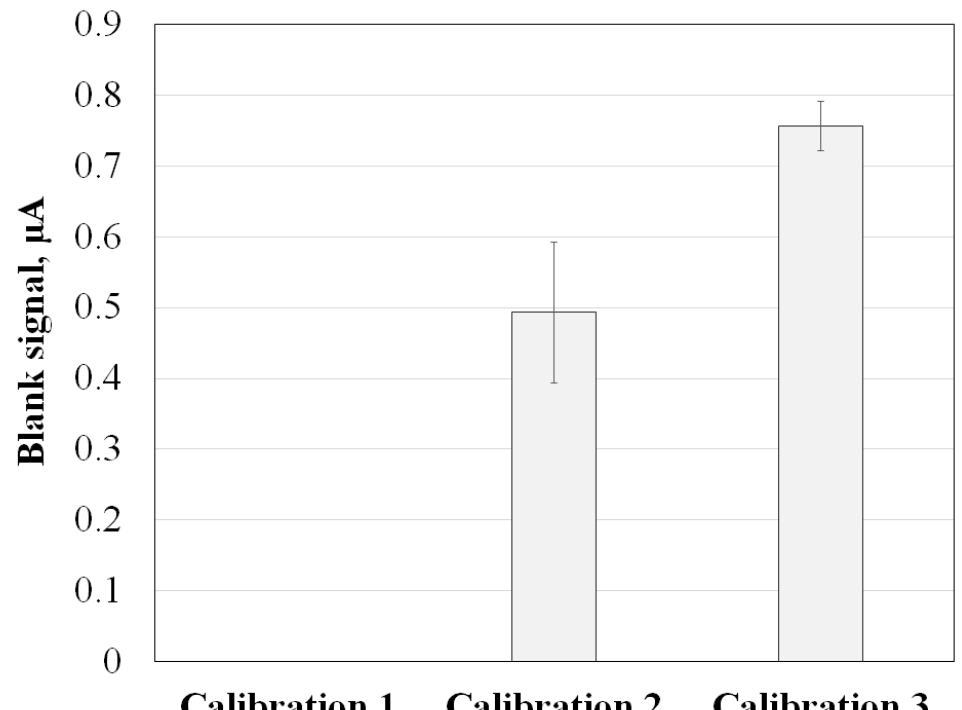

Figure 8.5. Repeatability assay. Variation of blank signal current over repeated calibrations of iodide. Error bars of associated deviation $(n=3)$. 
Table 8.3. Calibration parameters obtained through linear regression for the determination of iodide, using three different SPCE and CS/SPCE-based sensors.

\begin{tabular}{|c|c|c|c|c|c|c|}
\hline Sensors & $\begin{array}{l}\text { Sensitivity } \\
\left(\mu A \mathrm{mM}^{-1}\right)\end{array}$ & $R^{2}$ & $\begin{array}{c}\text { Sensitivity Average } \\
\left(\mu A \mathrm{mM}^{-1}\right)\end{array}$ & Linear Range & $\begin{array}{r}R S D \\
(\%)\end{array}$ & $\begin{array}{l}L O D \\
(M)\end{array}$ \\
\hline \multirow{3}{*}{ SPCE } & 23.46 & 0.996 & \multirow{3}{*}{23.68} & \multirow{3}{*}{$5 \mu \mathrm{M}$ to $7.5 \mathrm{mM}$} & \multirow{3}{*}{2.86} & \multirow{3}{*}{$2.9 \times 10^{-6}$} \\
\hline & 22.99 & 0.997 & & & & \\
\hline & 24.60 & 0.994 & & & & \\
\hline \multirow{3}{*}{$C S / S P C E$} & 342.55 & 0.995 & \multirow{3}{*}{336.18} & \multirow{3}{*}{$0.15 \mu \mathrm{M}$ to $500 \mu \mathrm{M}$} & \multirow{3}{*}{2.71} & \multirow{3}{*}{$1.0 \times 10^{-8}$} \\
\hline & 323.31 & 0.998 & & & & \\
\hline & 342.67 & 0.998 & & & & \\
\hline
\end{tabular}

\subsubsection{Construction of CS/SPCE for iodide determination}

In addition to the CSV technique, the use of a CS coating on the carbon working electrode surface can be valuable in determining trace concentrations of iodide. This polymer can improve the analytical performance, acting as a preconcentration component. Thus, the effect of CS was studied by covering the carbon working electrode surface with different quantities of a CS solution $(0.6 \%(\mathrm{w} / \mathrm{v})$ in $0.5 \%$ acetic acid). To do so, 0, 0.25, 0.50, 1.0 and $2.0 \mu \mathrm{L}$ of $\mathrm{CS}$ solution were deposited onto the working electrode and allowed to dry at room temperature. This new CS/SPCE-based sensor displays higher current outputs for the cathodic peak developed at $0.05 \mathrm{~V}$. The data obtained for $0.50,1.0$ and $2.0 \mu \mathrm{L}$ of deposited CS shows good linear coefficients and higher slopes for iodide determination. However, for 1.0 and $2.0 \mu \mathrm{L}$ of $\mathrm{CS}$, smaller concentrations of iodide could not be distinguished from the blank, possibly due to an excess of CS polymeric molecules, which prevents the redox process. Thus, $0.5 \mu \mathrm{L}$ of CS working electrode coating was selected as ideal, improving the ability of the CS/SPCE-based sensor to detect trace iodide (Figure 8.6). 


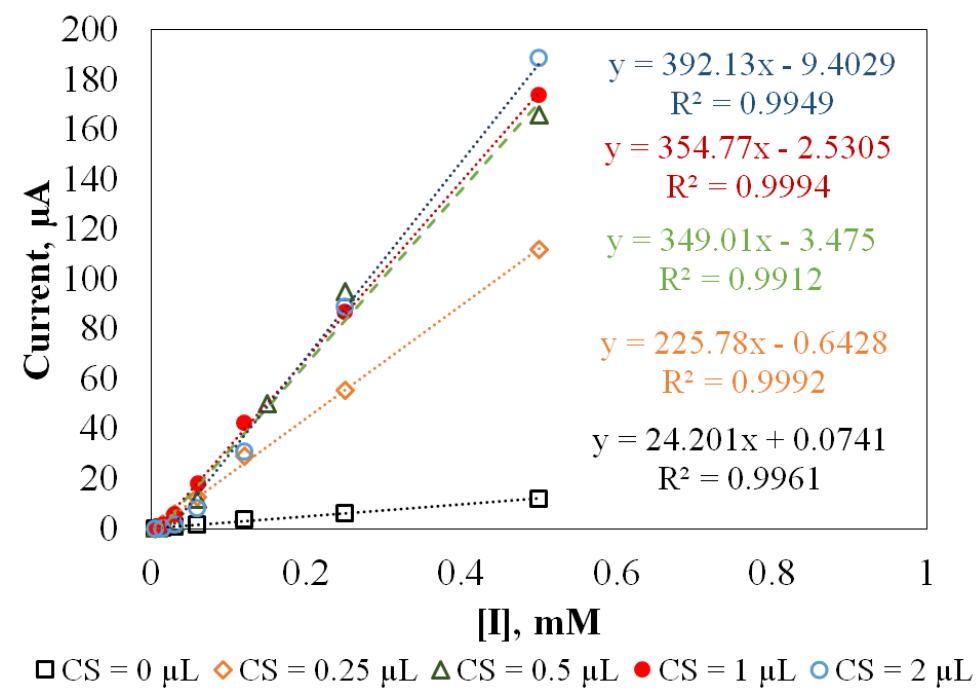

Figure 8.6. Effect of CS coating in iodide calibration curves. Tested iodide concentrations from 5 $\mu M$ to $500 \mu M$ in supporting electrolyte.

Different protonation states of the CS coating change the adsorptive properties of iodide, affecting the analytical signal. Accordingly, the effect of the supporting electrolyte $\mathrm{pH}$ was studied by observing the iodide cathodic peak current. Several PBS, with $\mathrm{pH}$ values of 5.5, $6.0,6.5,7.0,7.5,8.0$ and 8.5, were tested and the peak current for a $6.0 \mu \mathrm{M}$ KI sample was registered (Figure 8.7). The higher current output was achieved for a $\mathrm{pH}$ value of 6.5 of the PBS solution, reflecting a more positive interface, and consequently, an improved iodide adsorption. These experiments are consistent with the $\mathrm{pKa}$ value of the amino group of $\mathrm{CS}$, which is 6.5 [55]. Thus, the supporting electrolyte was used at $\mathrm{pH} 6.5$ to obtain an optimal iodide detection. Such $\mathrm{pH}$ variation, had no significant effect at SPCE-based sensor response to iodide, indicating that the electrochemical reaction is independent of protons (Figure 8.7). 


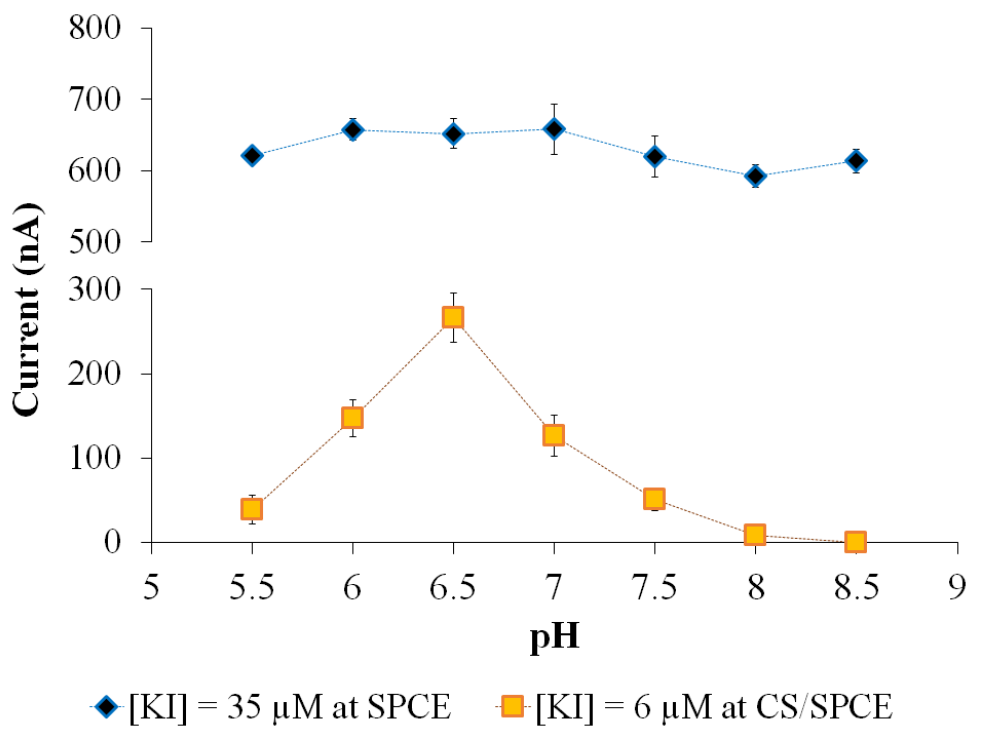

Figure 8.7. Effect of supporting electrolyte (PBS) $p H$, in iodide peak current for at $35 \mu M$ and 6 $\mu M$ of KI solution at SPCE and CS/SPCE, respectively. Error bars of associated deviation $(n=3)$.

Despite the improved electrochemical signals obtained using the CS/SPCE-based sensor, the analysis linear interval was reduced to a range from $0.15 \mu \mathrm{M}$ to $500 \mu \mathrm{M}$ (although this range could be extended by modifying the deposition time). After $500 \mu \mathrm{M}$, the beginning of a saturation plateau was observed (Figure 8.8). Nevertheless, this new range of analysis includes the UI range usually found in healthy individuals (> 6 years old) and pregnant women.

The CS/SPCE-based sensor was also characterized in terms of reproducibility. In figure 8.9, the voltammetric signals developed at CS/SPCE are shown, exhibiting improved and stable peaks. The analytical parameters, summarized in Table 8.3, show a slight improvement in RSD\% value for CS/SPCE-based sensors. However, the most noteworthy changes are in the sensitivity, which is approximately 14 times higher $\left(336.18 \mu \mathrm{A} \mathrm{mM}^{-1}\right)$ compared to the SPCE-based sensor, and also in the LOD value of $1.0 \times 10^{-8} \mathrm{M}\left(S_{x / y}\right.$ for $0.15 \mu \mathrm{M})$, demonstrating its potential use as a tool for the analysis of matrices with trace iodide content. 


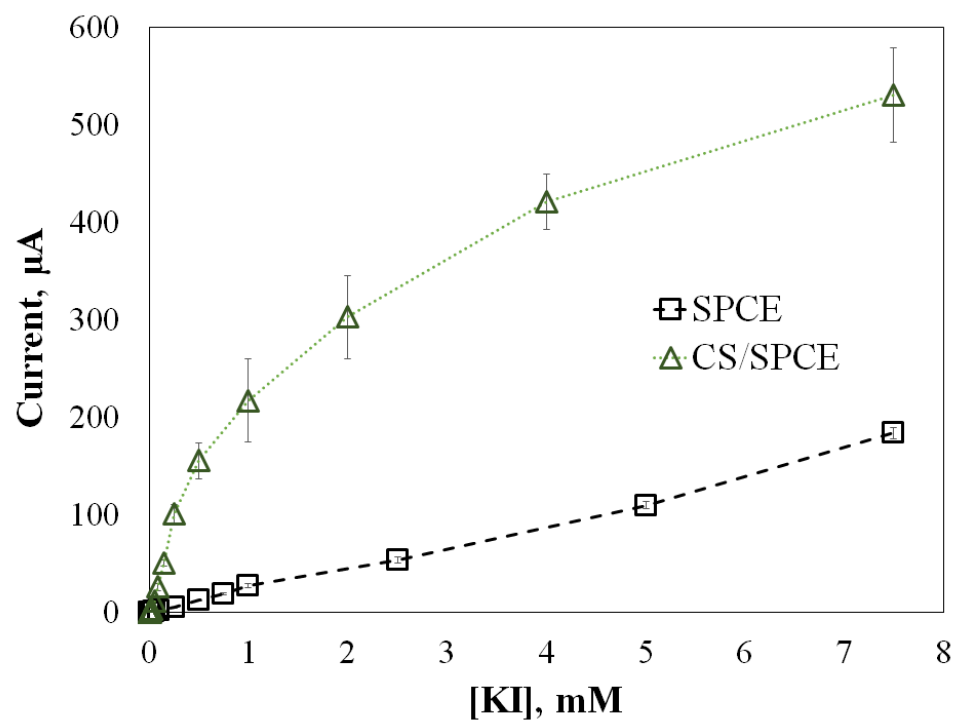

Figure 8.8. Iodide calibration at SPCE and CS/SPCE - based sensors. Error bars of associated deviation $(n=3)$.

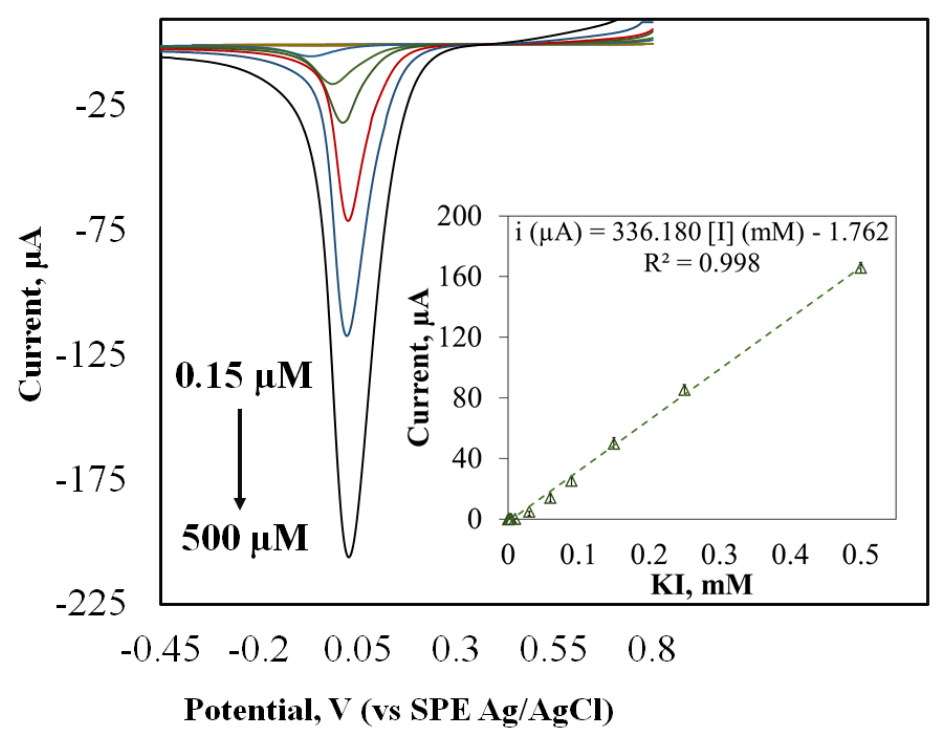

Figure 8.9. CSV peaks recorded at CS/SPCE-based sensor of a $150 \mu L$ drop of supporting electrolyte containing KI ranging from $0.15 \mu \mathrm{M}$ to $50 \mu \mathrm{M}$. Inset: Average calibration curve and associated deviation $(n=3)$. 
Finally, similar to the observed for the SPCE-based sensor, after the first iodide calibration (15 concentration measurements), a peak was also found in the CS/SPCE blank run, demonstrating its applicability for a single calibration.

\subsubsection{Study of interferences}

The selectivity of the sensors for iodide determination was demonstrated, conducting CSV measurements of different solutions containing possible interfering species for a constant concentration of the anion. Regarding the range of analysis offered by both sensors, iodide concentrations of $500 \mu \mathrm{M}$ and $25 \mu \mathrm{M}$, were respectively selected to study the effect of foreign species using SPCE and CS/SPCE-based sensors. Several compounds, such as other halides and components present in urine, were considered in this study (Table 8.4).

The iodide cathodic peak current was evaluated when fluoride, chloride and bromine are present in the matrix. Fluoride $(1 \mathrm{mM})$ and bromide $(1 \mathrm{mM})$ were seen to have no effect on sensors performances, developing a peak current close to the $100 \%$ expected for the iodide concentrations tested. Chloride was studied differently, since it is present in much higher concentrations in the supporting electrolyte than iodide and is therefore not considered as interference at this concentration level $(10 \mathrm{mM})$. However, the levels of chloride anions usually reach concentrations of $100 \mathrm{mM}$ in biological matrices, and so the effect of such high concentrations was studied. The presence of $100 \mathrm{mM}$ of chloride anions when using the SPCE-based sensor was unnoticeable, resulting in the expected iodide peak current output. However, the effect is considerable when using the CS/SCPE-based sensor, decreasing to approximately $18 \%$ of the expected iodide signal. At these high amounts, chloride anions are preferably adsorbed onto the CS membrane, avoiding iodide preconcentration at the working electrode, inhibiting the cathodic voltammetric peak generation. The iodide signal was fully recovered with the CS/SPCE-based sensor, when chloride concentration is decreased to $30 \mathrm{mM}$, returning the expected iodide cathodic peak current. 
Table 8.4. Effect of the concentration of interfering species in the iodide peak current percentage $\left(\% I_{p}\right)$. Average value obtained for triplicates. (NA - not analyzed $)$

\begin{tabular}{|c|c|c|c|}
\hline \multirow{2}{*}{ Interference } & \multirow{2}{*}{ [Interference] } & \multicolumn{2}{|c|}{$\% I_{p}(\mu A)$} \\
\hline & & $\operatorname{SPCE}\left(\left[I^{-}\right]=500 \mu M\right)$ & $C S / S P C E\left(\left[I^{-}\right]=25 \mu M\right)$ \\
\hline No interference & - & 100 & 100 \\
\hline $\mathrm{F}^{-}$ & $1 \mathrm{mM}$ & $97.93 \pm 3.13$ & $100.37 \pm 0.75$ \\
\hline \multirow{2}{*}{$\mathrm{Cl}^{-}$} & $30 \mathrm{mM}$ & NA & $98.74 \pm 2.12$ \\
\hline & $100 \mathrm{mM}$ & $99.95 \pm 3.92$ & $17.54 \pm 4.56$ \\
\hline $\mathrm{Br}^{-}$ & $1 \mathrm{mM}$ & $96.21 \pm 4.92$ & $97.5 \pm 1.93$ \\
\hline \multirow{2}{*}{ Ascorbic Acid } & $0.2 \mu \mathrm{M}$ & NA & $96.87 \pm 2.48$ \\
\hline & $1 \mu \mathrm{M}$ & $100.68 \pm 4.05$ & $17.31 \pm 10.71$ \\
\hline \multirow{2}{*}{ Ammonia } & $1.33 \mathrm{mM}$ & NA & $98.89 \pm 0.99$ \\
\hline & $4 \mathrm{mM}$ & $99.74 \pm 2.57$ & $34.68 \pm 0.78$ \\
\hline Creatinine & $4 \mathrm{mM}$ & $105.41 \pm 2.40$ & $96.78 \pm 1.57$ \\
\hline \multirow{2}{*}{ Glucose } & $0.5 \mu \mathrm{M}$ & NA & $96.96 \pm 3.11$ \\
\hline & $1 \mu \mathrm{M}$ & $96.52 \pm 2.68$ & $53.53 \pm 0.43$ \\
\hline Uric Acid & $1 \mu \mathrm{M}$ & $104.22 \pm 1.61$ & $97.60 \pm 0.67$ \\
\hline Urea & $100 \mu \mathrm{M}$ & $98.267 \pm 4.01$ & $96.24 \pm 2.69$ \\
\hline
\end{tabular}

Similarly, the effect of various urine constituents [72] such as, ascorbic acid $(1 \mu \mathrm{M})$, ammonia $(4 \mathrm{mM})$, creatinine $(4 \mathrm{mM})$, glucose $(1 \mu \mathrm{M})$, urea $(100 \mu \mathrm{M})$ and uric acid $(1 \mu \mathrm{M})$, was also evaluated for iodide determination. The behavior of interfering species differs depending on the sensor used. In the case of the SPCE-based sensor, the determination of iodide is not affected. On the other hand, while creatinine, uric acid and urea have no effect on iodide determination when using the CS/SPCE-based sensor, it was observed that ascorbic acid, ammonia and glucose do indeed affect sensor performance, requiring a dilution to fully recover the predicted iodide cathodic peak current.

This study proves that, despite the lack of sensitivity of the SPCE-based sensor, the device exhibits a high selectivity for iodide determination. On the other hand, the modification of the working electrode with CS, used to improve the device's analytical performance, affects 
the selectivity of the sensor. This results in the need for sample pretreatment before UI analysis when using a CS/SPCE-based sensor.

Given the performances and properties described above, the developed sensors exhibit an accurate sensitivity and suitability for the analysis of a wide variety of real-life samples.

\subsubsection{Sensors validation}

To further validate these sensors, three types of urine samples, tap water, and two pharmaceutical pills containing iodide, were used. The results, summarized in Table 8.7, were either corroborated using the SK spectrophotometric method (Figure 8.10 and Table $8.5)$, or through the recovery value in the case of spiked samples. The results of the pharmaceutical formulations were also verified with the iodide values indicated on the product labels.

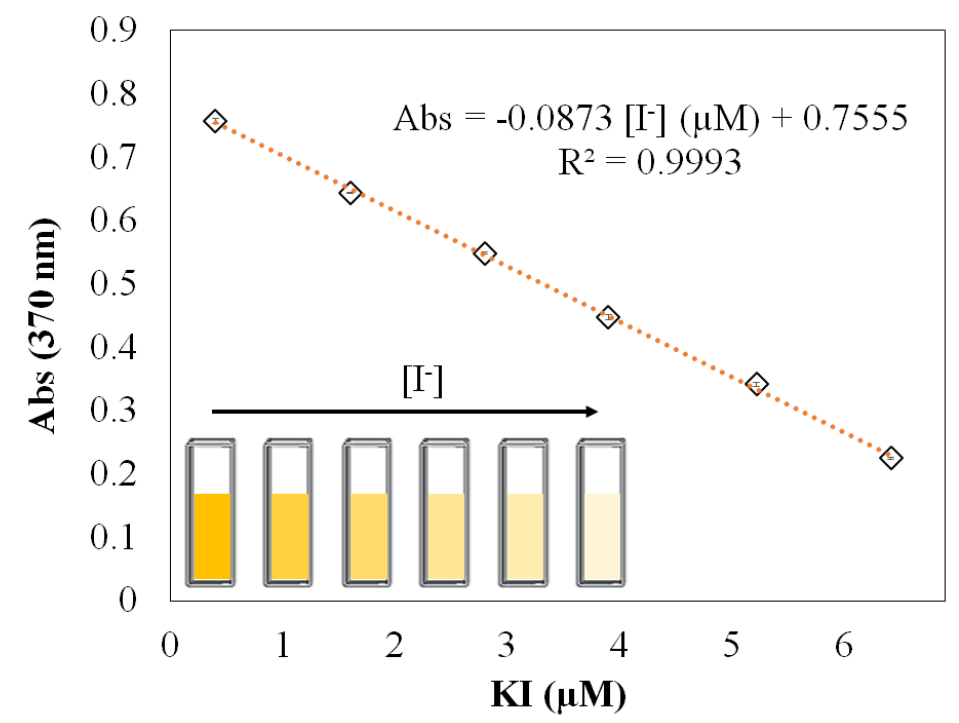

Figure 8.10. Average iodide calibration curve of three independent assays of SK based spectrophotometric method at $370 \mathrm{~nm}$. Error bars corresponding to standard deviation. 
Table 8.5. Calibration parameters obtained through linear regression for the determination of iodide, using SK-based spectrophotometric method.

\begin{tabular}{|c|c|c|c|c|c|c|}
\hline $\begin{array}{l}\text { Replicas } \\
(370 \mathrm{~nm})\end{array}$ & $\begin{array}{c}\text { Slope } \\
\left(\text { Abs } \mu \mathrm{M}^{-1}\right)\end{array}$ & $R^{2}$ & $\begin{array}{c}\text { Slopes average } \\
\left(\text { Abs } \mu \mathrm{M}^{-1}\right)\end{array}$ & Linear Range & $\begin{array}{l}R S D \\
(\%)\end{array}$ & $\begin{array}{l}L O D \\
(\mu M)\end{array}$ \\
\hline Assay 1 & 0.0878 & 0.999 & & & & \\
\hline Assay 2 & 0.0864 & 0.999 & 0.0873 & $0 \mu \mathrm{M}-6 \mu \mathrm{M}$ & 0.71 & 0.155 \\
\hline Assay 3 & 0.0876 & 0.999 & & & & \\
\hline
\end{tabular}

\subsubsection{UI determination}

Following the standard addition method, electrochemical determination of urinary iodide (UI) was performed by drop-casting $150 \mu \mathrm{L}$ of urine onto the sensor's working area. After that, spiked urine samples were also measured, and the peak currents obtained were used to construct the calibration curves.

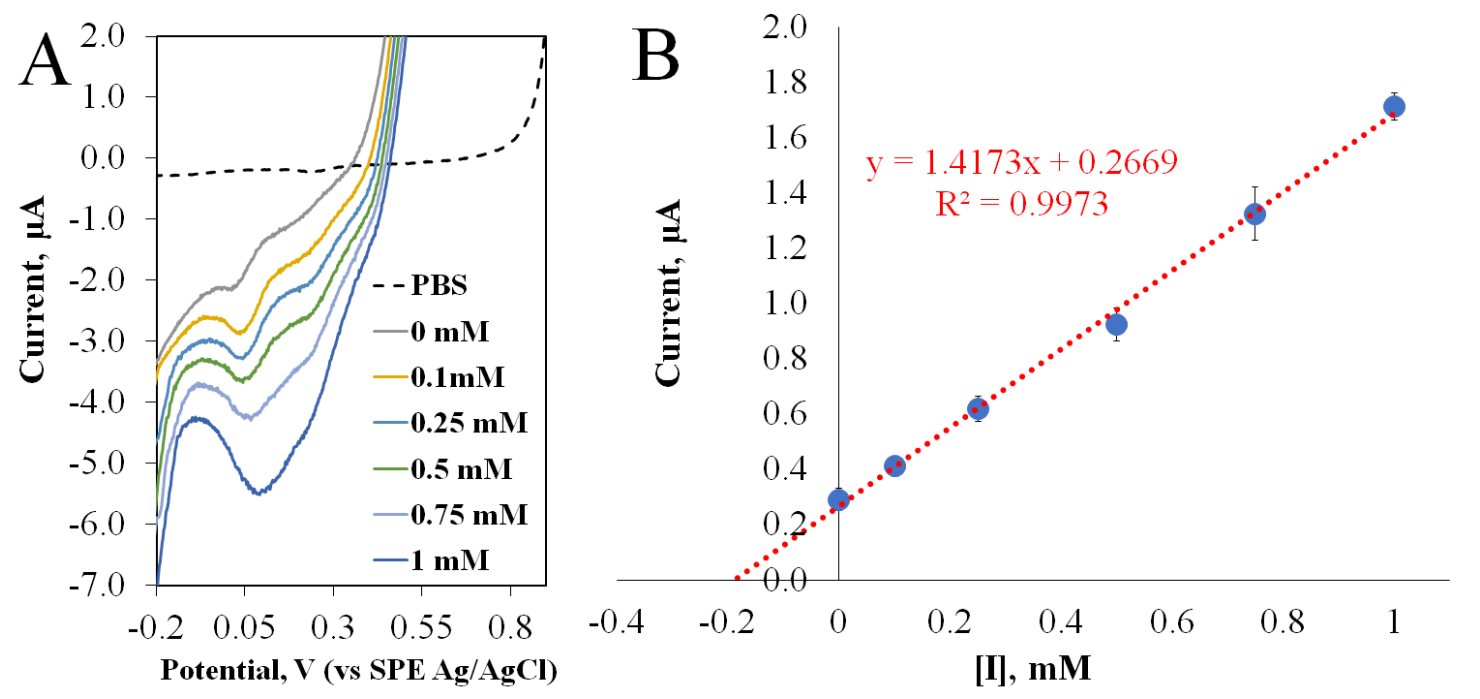

Figure 8.11. Urine iodide electrochemical determination. (A) CSV peaks recorded at SPCE-based sensor for raw urine, collected 2 hours after administration of intravenous iodinated contrast; $(B)$ average calibration curve of standard addition method and associated deviation $(n=3)$. 
The applicability of the proposed sensors was checked by analyzing urine samples collected from three types of subjects: a patient submitted to intravenous administration of iodinated contrast, a healthy individual and an individual with an iodide deficit.

Firstly, a urine sample collected after 2 hours of intravenous administration of iodinated contrast was tested using the SPCE-based sensor. A small cathodic peak, which is increased in spiked urine, was observed around $0.05 \mathrm{~V}$ (vs SPE $\mathrm{Ag} / \mathrm{AgCl}$ ). The slope obtained for this raw sample $\left(1.14 \mu \mathrm{A} \mathrm{mM}^{-1}\right)$ shows less sensitivity than the one observed in PBS (23.68 $\mu \mathrm{A} \mathrm{mM}^{-1}$ ), which is due to the matrix effect (Figure 8.11). Nevertheless, as shown in table 8.7 , the iodide content $(0.192 \pm 0.032 \mathrm{mM})$ determined for this raw sample proves to be well correlated with the value obtained through the SK spectrophotometric method $(0.206$ $\pm 0.017 \mathrm{mM}$ ). It should be noted that the amount of iodide found in this sample is considerably higher than what has been described for urine samples of healthy individuals discussed in the literature. The high doses of iodide administered for a radiological diagnosis explains the increased value found for this sample.

Additionally, the proposed SPCE-based sensor also proves to be an efficient analytical tool for monitoring urine iodide elimination over time. A comparative study of the cathodic peak current developed for urine samples collected at different times after intravenous iodinated contrast presents a lower value over time (Figure 8.12).

The urine samples of healthy and iodide deficient individuals were analyzed using the CS/SPCE-based sensor, since the sensitivity achieved by this device is suitable for determining the small iodide concentrations expected to be present in these samples. When the electrochemical measurements were performed using the CS/SPCE-based sensor, no signal was observed for the raw urine, nor when it was spiked with iodide concentrations. As predicted from the results of the interference study, iodide determination is not possible given the matrix effect. To avoid such interferences, both urine samples were submitted to the same pretreatment used for the spectrophotometric method and the resulting samples were measured by CSV using the CS/SCPE-based sensor. 


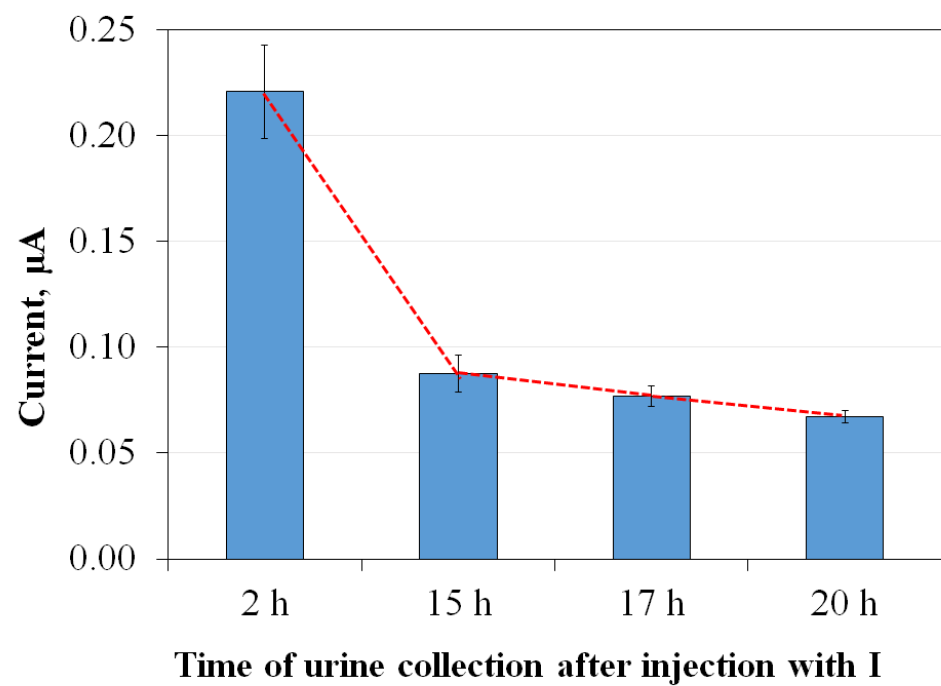

Figure 8.12. Iodide clearance from organism. Peak current intensity variation with the time of urine collection, registered at SPCE-based sensor. Error bars of associated deviation $(n=3)$.

For the pretreated urine obtained from the healthy subject, a UI of $4.22 \pm 0.22 \mu \mathrm{M}$ was found, which is in accordance with the values established in the WHO's guidelines. The UI of this sample was validated using the SK spectrophotometric method that determines a UI of $4.21 \pm 0.01 \mu \mathrm{M}$ (Figure 8.13. A).

Finally, a urine sample from an iodide deficient patient was also evaluated. Since no iodide was detected using the electrochemical or spectrophotometric methods, the raw urine was spiked with $1.50 \mu \mathrm{M}$ of $\mathrm{KI}$ and the determination was performed in order to confirm the results. The sensor performed suitably, recovering $104.33 \%$ of the spiked value, an iodide content of $1.57 \pm 0.23 \mu \mathrm{M}$ (Figure 8.13. B).

Regarding the results obtained for urine analysis using the CS/SPCE-based sensor, this method requires a simple extra step of sample pretreatment prior to iodide determination. Despite that, the sensor proves to be highly suitable for anion determination in this matrix. 

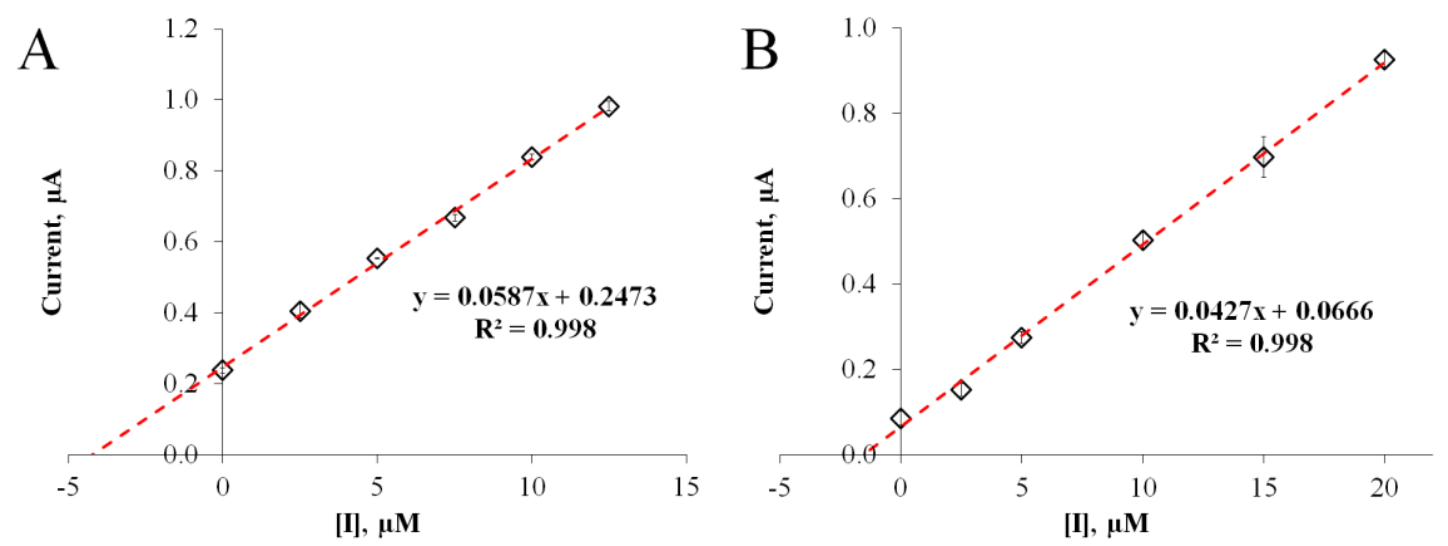

Figure 8.13. Average curves obtained using the standard addition method for iodide determination in urines from: (A) healthy subject and (B) iodide deficient subject. Error bars of associated deviation $(n=3)$.

\subsubsection{Tap water analysis}

A tap water sample was analyzed without the sample pretreatment step and using the CS/SCPE-based sensor. The device shows no peak for the sample, reflecting the absence of iodide in the matrix. After spiking the sample with $2.50 \mu \mathrm{M}$ of $\mathrm{KI}$, the standard addition method was performed. The analysis showed a shift in the cathodic peak; however, the sensor proves to be able to determine an iodide content of $2.52 \pm 0.40 \mu \mathrm{M}$, exhibiting a good recovery (100.60\%) (Figure 8.14).

\subsubsection{Iodide containing pills}

Two types of iodide containing pills, Yodocefol ${ }^{\circledR}$ and Supradyn ${ }^{\circledR}$, were also analyzed using the electrochemical method. The standard addition method results yielded iodide values according to the labeled concentration provided by the commercial supplier (Figure 8.15). The SK spectrophotometric method was also applied as a reference method. However, this reference method can only be applied in the analysis of Yodocefol ${ }^{\circledR}$. Concerning the 
spectrophotometric results obtained at $370 \mathrm{~nm}$ for Supradyn ${ }^{\circledR}$, an insignificant absorbance variation with time was found (Table 8.6). These results show that iodide determination in Supradyn ${ }^{\circledR}$ using spectrophotometric analysis yields anomalous results. This is because the formulation contains a mixture of several minerals, vitamins and coenzymes, which interfere in the iodide spectrophotometric determination. Despite this, the SPCE-based sensor results correlated well with the labeled iodide concentration, making it suitable for iodide determination in this kind of sample.

In conclusion, an adequate analytical performance and linearity were obtained in both analyses, offering an accurate, simple and low-cost method of controlling the iodide content of these pharmaceutical formulations.
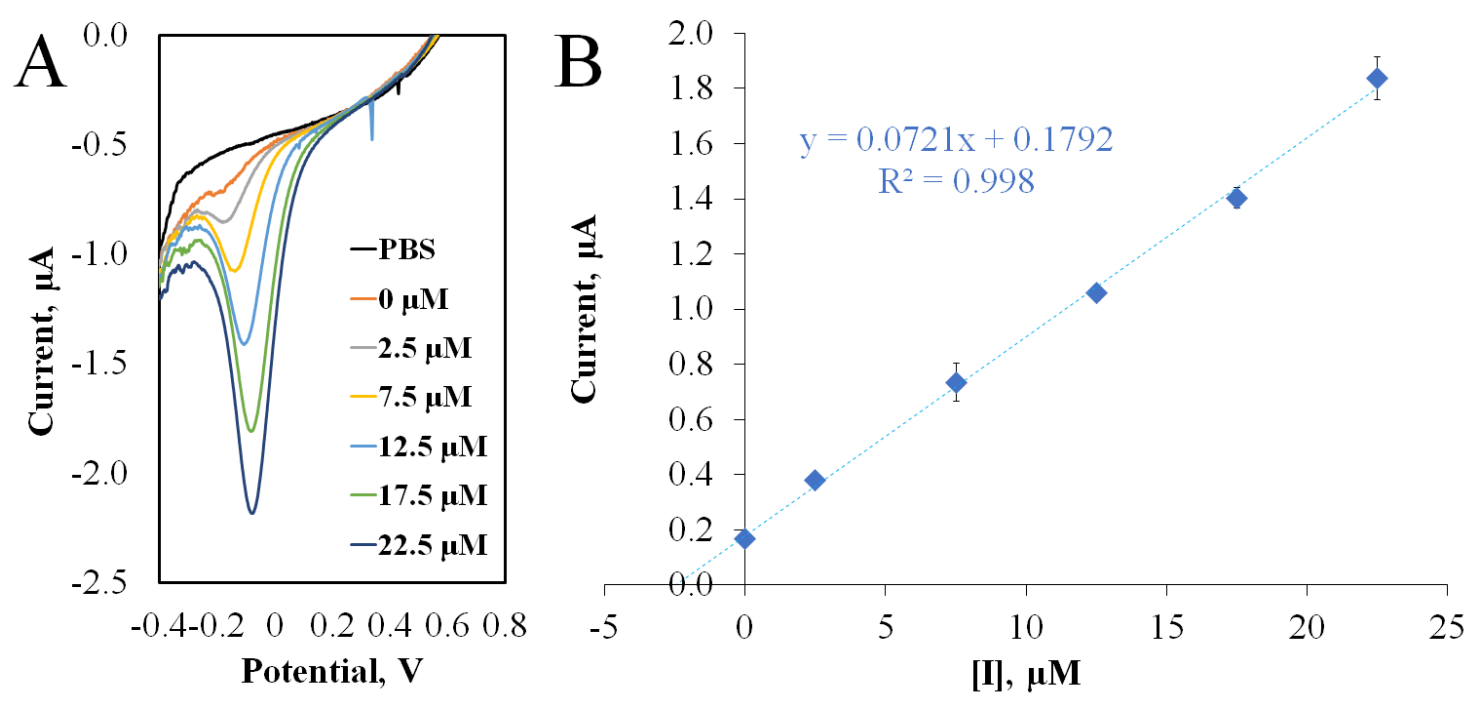

Figure 8.14. Iodide CS/SPCE-based sensor response obtained in tap water. (A) CSV peaks recorded in a $150 \mu \mathrm{L}$ drop of tap water with iodide ranging from 0 to $22.5 \mu \mathrm{M}$; and (B) Average calibration curve of standard addition method and error bars of associated deviation $(n=3)$. 

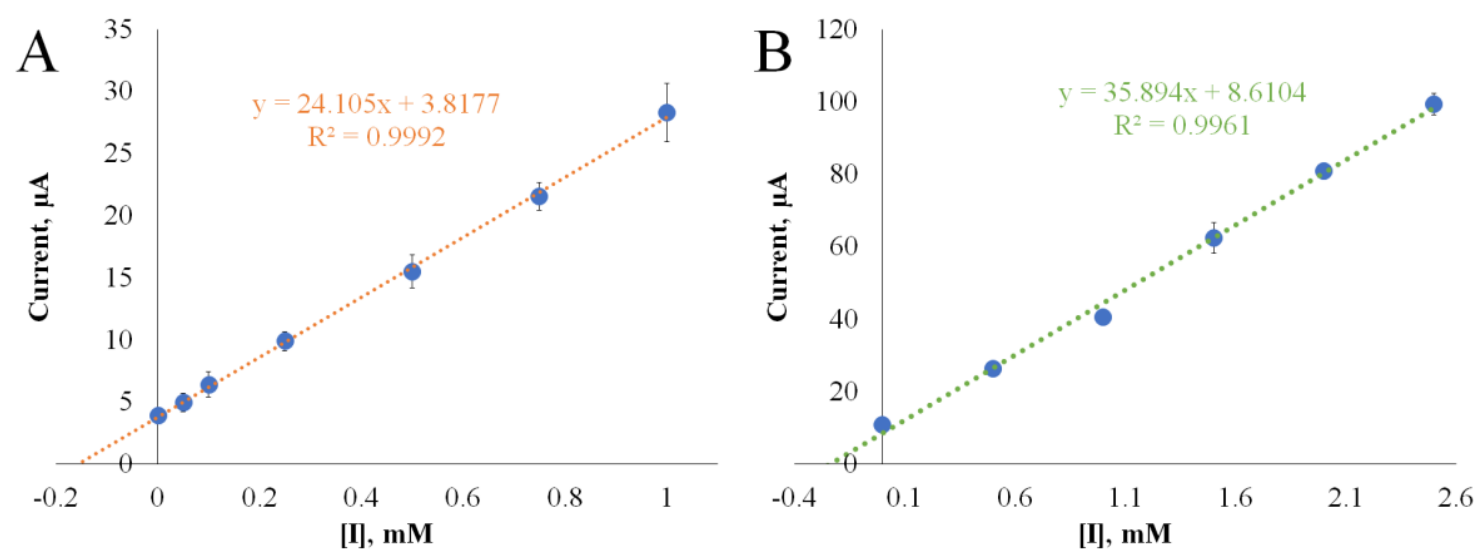

Figure 8.15. Average curves obtained using the standard addition method for iodide determination in (A) Yodocefol ${ }^{\circledR}$ and (B) Supradyn ${ }^{\circledR}$. Error bars of associated deviation ( $\left.n=3\right)$.

Table 8.6. Variation of absorbance value at $370 \mathrm{~nm}$, of $\operatorname{Supradin}^{\circledR}$ and $\mathrm{Yodocefol}^{\circledR}$, with the time of spectrophotometric measurement at 30, 60 and $90 \mathrm{~s}$.

\begin{tabular}{cccc}
\hline \multirow{2}{*}{ Pharmaceutical samples } & \multicolumn{3}{c}{ Abs (370 $\mathbf{n m})$} \\
\cline { 2 - 4 } & $\mathbf{3 0} \boldsymbol{s}$ & $\mathbf{6 0} \mathbf{s}$ & $\mathbf{9 0} \boldsymbol{s}$ \\
\hline Supradyn $^{\circledR}$ & 0.743 & 0.729 & 0.717 \\
Yodocefol $^{\circledR}$ & 0.802 & 0.747 & 0.714 \\
\hline
\end{tabular}

Table 8.7. Voltammetric sensors and spectrophotometric analysis results of iodide in urine, tap water, Supradyn ${ }^{\circledR}$ and Yodocefol ${ }^{\circledR}$ obtained using the standard addition method $(n=3)$.

\begin{tabular}{|c|c|c|c|c|c|}
\hline Samples & $\begin{array}{c}\text { Spike } \\
I^{-}(\mu M)\end{array}$ & $\begin{array}{c}\text { Electrochemica } \\
\text { l sensors }\end{array}$ & $\begin{array}{c}\text { SK- } \\
\text { spectrophotometric } \\
\text { method }\end{array}$ & $\begin{array}{l}\text { Labeled } \\
I(m M)\end{array}$ & $\begin{array}{c}\text { Recovery } \\
(\%)\end{array}$ \\
\hline Urine (2 hours) ${ }^{a}$ & - & $0.19 \pm 0.03 \mathrm{mM}$ & $0.21 \pm 0.02 \mathrm{mM}$ & - & - \\
\hline Healthy Urine ${ }^{b}$ & - & $4.22 \pm 0.22 \mu \mathrm{M}$ & $4.21 \pm 0.01 \mu \mathrm{M}$ & - & - \\
\hline Iodide deficient urine ${ }^{\mathrm{b}, \#}$ & 1.50 & $1.57 \pm 0.23 \mu \mathrm{M}$ & - & - & 104.33 \\
\hline Tap water ${ }^{b, \#}$ & 2.50 & $2.52 \pm 0.40 \mu \mathrm{M}$ & - & - & 100.60 \\
\hline Supradyn $^{\circledR}{ }^{\circledR}$ & - & $0.24 \pm 0.03 \mathrm{mM}$ & $0.12 \pm 0.01 \mathrm{mM}^{*}$ & 0.24 & - \\
\hline Yodocefol $^{\circledR \text { a }}$ & - & $0.16 \pm 0.02 \mathrm{mM}$ & $0.13 \pm 0.06 \mathrm{mM}$ & 0.16 & - \\
\hline
\end{tabular}

a - SPCE-based sensor analysis; ${ }^{b}$ - CS/SPCE-based sensor analysis; ${ }^{\#}$ - Iodide not detected in the samples; * - Value not validated by SK - spectrophotometric method. 


\subsection{Conclusions}

In this work, two new, simple, easy-to-use, disposable, reproducible and low-cost sensors were developed to determine iodide in real-life samples. Firstly, highly selective and widerange iodide analysis was achieved, through the careful control of CSV experimental parameters performed using a SPCE-based sensor. This device exhibits limitations when used in matrices with trace iodide concentrations. Therefore, a second CS/SPCE-based sensor was constructed to increase the cathodic peak current intensity for iodide. The modification of the working electrode with a CS coating, together with the chosen CSV conditions, favored the preconcentration of iodide anions on the positively charged working electrode surface. The thickness of the CS coating and the $\mathrm{pH}$ value of the supporting electrolyte prove to be important variables for the analytical performance of this sensor. This modification offers an improved detection limit of $10 \mathrm{nM}$, about 100 times less than that obtained for the SCPE-based sensor, with 14 times more sensitivity which makes it a suitable device for monitoring iodide in urine samples. However, this modification results in the presence of interfering species, requiring a simple pretreatment before the analysis in the case of urine samples.

Despite the need for one additional step when using the CS/SPCE-based sensor, both devices present several advantages to those described in the literature, such as the lowest LOD value obtained and an adequate range for urine iodide analysis. Moreover, the wide range of analysis found when using the SPCE-based sensor makes it a versatile tool for the selective, accurate and reproducible determination of iodide in different kinds of samples. The advantages achieved with the CS/SPCE-based sensor for trace iodide determination, make this device a promising analytical tool specially interesting for urinary iodide monitoring. 


\subsection{REFERENCES}

[1] M. Haap, H.J. Roth, T. Huber, H. Dittmann, R. Wahl, Urinary iodine: Comparison of a simple method for its determination in microplates with measurement by inductivelycoupled plasma mass spectrometry, Sci. Rep. 7 (2017) 1-10.

[2] WHO, Assessment of the iodine deficiency disorders and monitoring their elimination, 2007.

[3] B. de Benoist, M. Andersson, I. Egli, B. Takkouche, H. Allen, Iodine status worldwide, 2004.

[4] M.B. Zimmermann, Iodine Deficiency, Endocr. Rev. 30 (2009) 376-40.

[5] O. Chailapakul, M. Amatatongchai, P. Wilairat, K. Grudpan, D. Nacapricha, Flowinjection determination of iodide ion in nuclear emergency tablets, using boron-doped diamond thin film electrode, Talanta. 64 (2004) 1253-1258.

[6] H.S. Toh, K. Tschulik, C. Batchelor-McAuley, R.G. Compton, Electrochemical quantification of iodide ions in synthetic urine using silver nanoparticles: A proof-ofconcept, Analyst. 139 (2014) 3986-3990.

[7] J. Mihailović, Current concepts of 131I therapy in oncology: Indications, methods and follow up, Arch. Oncol. 14 (2006) 45-51.

[8] C. Durante, N. Haddy, E. Baudin, S. Leboulleux, D. Hartl, J.P. Travagli, B. Caillou, M. Ricard, J.D. Lumbroso, F. De Vathaire, M. Schlumberger, Long-term outcome of 444 patients with distant metastases from papillary and follicular thyroid carcinoma: Benefits and limits of radioiodine therapy, J. Clin. Endocrinol. Metab. 91 (2006) 2892-2899.

[9] E. Hindie, P. Zanotti-Fregonara, I. Keller, F. Duron, J.-Y. Devaux, M. CalzadaNocaudie, E. Sarfati, J.-L. Moretti, P. Bouchard, M.-E. Toubert, Bone metastases of 
differentiated thyroid cancer: impact of early ${ }^{131}$ I-based detection on outcome, Endocr. Relat. Cancer. 14 (2007) 799-807.

[10] E.C. Nijssen, P.J. Nelemans, R.J. Rennenberg, V. Van Ommen, J.E. Wildberger, Evaluation of Safety Guidelines on the Use of Iodinated Contrast Material: Conundrum Continued, Invest. Radiol. 53 (2018) 616-622.

[11] C.S. Ng, S.P. Kalva, C. Gunnarsson, M.P. Ryan, E.R. Baker, R.L. Mehta, Risk of renal events following intravenous iodinated contrast material administration among inpatients admitted with cancer a retrospective hospital claims analysis, Cancer Imaging. 18 (2018) 1-14.

[12] C.P. Shelor, P.K. Dasgupta, Review of analytical methods for the quantification of iodine in complex matrices, Anal. Chim. Acta. 702 (2011) 16-36.

[13] E.B. Sandell, I.M. Kolthoff, Micro determination of iodine by a catalytic method, Mikrochim. Acta. 1 (1937) 9-25.

[14] W. Zhang, A. Mnatsakanov, R. Hower, H. Cantor, Y. Wang, Urinary iodine assays and ionophore based potentiometric iodide sensors, Front. Biosci. 10 (2005) 88-93.

[15] J. Melichercik, L. Szijarto, A. R. Hill, Comparison of ion-specific electrode and high performance liquid chromatography methods for the determination of iodide in milk., J. Dairy Sci. 89 (2006) 934-7.

[16] M.C. Yebra, M.H. Bollaín, A simple indirect automatic method to determine total iodine in milk products by flame atomic absorption spectrometry, Talanta. 82 (2010) 828833.

[17] B. Michalke, P. Schramel, Iodine speciation in biological samples by capillary electrophoresis-inductively coupled plasma mass spectrometry., Electrophoresis. 20 (1999) 2547-2553.

[18] E. Vtorushina, I. Saprykin, G. Knapp, Optimization of the conditions of oxidation vapor generation for determining chlorine, bromine, and iodine in aqueous solutions by 
inductively coupled plasma atomic-emission spectrometry, J. Anal. Chem. 63 (2008) 643648.

[19] K. Isaac-Olive, T.T. Kyaw, A. Chatt, Estimation of anthropogenic organo-chlorine, bromine and iodine compounds in apolar lipid fractions of bovine milk by solid-phase extraction and neutron activation analysis (SPE-NAA), J. Radioanal. Nucl. Chem. 8 (2018).

[20] V. V. Kuznetsov, Y. V. Ermolenko, L. Seffar, Amylose and amylopectin as reagents for the flow-injection determination of elemental iodine, J. Anal. Chem. 62 (2007) 479485.

[21] L. Rong, L.W. Lim, T. Takeuchi, Rapid determination of iodide in seawater samples by ion chromatography with chemically-bonded vitamin-U stationary phase, Microchem. J. 108 (2013) 113-116.

[22] P. Das, M. Gupta, A. Jain, K.K. Verma, Single drop microextraction or solid phase microextraction-gas chromatography-mass spectrometry for the determination of iodine in pharmaceuticals, iodized salt, milk powder and vegetables involving conversion into 4iodo-N,N-dimethylaniline, J. Chromatogr. A. 1023 (2004) 33-39.

[23] T. Tomiyasu, M. Nonaka, M. Uchikado, K. Anazawa, H. Sakamoto, Kinetic determination of total iodine in urine and foodstuffs using a mixed acid as a pretreatment agent, Anal. Sci. 20 (2004) 391-3.

[24] E.A. Crecelius, Determination of Total Iodine in Milk by X-Ray Fluorescence Spectrometry and Iodide Electrode, Anal. Chem. 47 (1975) 2034-2035.

[25] P. Ünak, Ş. Darcan, F. Yurt, Z. Biber, M. Çoker, Determination of iodide amounts in urine and water by isotope dilution analysis, Biol. Trace Elem. Res. 71-72 (1999) 463470.

[26] S.-Z. Yao, P. Chen, W.-Z. Wei, A quartz crystal microbalance method for the determination of iodine in foodstuffs, Food Chem. 67 (1999) 311-316. 
[27] F.C. de Souza, C.G. Vegas, D.A.I. da Silva, M.S. Ribeiro, M.F. Cabral, M.A. de Melo, R.M.T. Mattos, R.B. Faria, E. D’Elia, Amperometric and potentiometric determination of iodide using carbon electrodes modified with salophen complex, J. Electroanal. Chem. 783 (2016) 49-55.

[28] L. Yang, L. Zou, G. Li, B. Ye, Simple and rapid determination of trace iodide by cathodic stripping voltammetry, Talanta. 147 (2016) 634-640.

[29] K. Nakagawa, K. Suzuki, M. Kondo, S. Hayakawa, M. Nakayama, Electrosynthesis of Layered Organo-Manganese Dioxide Framework-Doped with Cobalt for Iodide Sensing, Langmuir. 33 (2017) 4647-4653.

[30] A. Shirmardi, M. Shamsipur, M. Akhond, J. Monjezi, Electronic tongue for simultaneous determination of cyanide, thiocyanate and iodide, Meas. J. Int. Meas. Confed. 88 (2016) 27-33.

[31] T.W. Chen, T.H. Tsai, S.M. Chen, K.C. Lin, Using PEDOT film modified electrode to monitor iodide and its enhancement of arsenite sensing, Int. J. Electrochem. Sci. 6 (2011) 2043-2057.

[32] L. Tian, L. Liu, L. Chen, N. Lu, H. Xu, Electrochemical determination of iodide on a vanadium oxide-polypropylene carbonate coated glassy carbon electrode, Talanta. 66 (2005) 130-135.

[33] I.S. El-Hallag, Electrochemical oxidation of iodide at a glassy carbon electrode in methylene chloride at various temperatures, J. Chil. Chem. Soc. 55 (2010) 67-73.

[34] X. Wu, G. Lu, X. Zhang, X. Yao, Anodic voltammetric behavior of iodide at a chitosan-modified glassy carbon electrode, Anal. Lett. 34 (2001) 1205-1214.

[35] F.M. Abdel-Haleem, O.R. Shehab, Comparative Study of Carbon Paste, Screen Printed, and PVC Potentiometric Sensors Based on Copper-sulphamethazine Schiff Base Complex for Determination of Iodide - Experimental and Theoretical Approaches, Electroanalysis. 28 (2016) 800-807. 
[36] E. Cho, A. Perebikovsky, O. Benice, S. Holmberg, M. Madou, M. Ghazinejad, Rapid iodine sensing on mechanically treated carbon nanofibers, Sensors (Switzerland). 18 (2018) 1-9.

[37] B. Dielacher, R.F. Tiefenauer, J. Junesch, J. Vörös, Iodide sensing via electrochemical etching of ultrathin gold films, Nanotechnology. 26 (2015).

[38] Z.H. Ibupoto, K. Khun, M. Willander, A selective iodide ion sensor electrode based on functionalized ZnO nanotubes, Sensors (Switzerland). 13 (2013) 1984-1997.

[39] Y. Zhang, M. Wang, Chitosan/Colloid Au Modified Gold Film Microelectrode for Iodide Determination, Int. J. Electrochem. Sci. 8 (2013) 4595-4609.

[40] D.S. Silvester, E.I. Rogers, L.E. Barrosse-Antle, T.L. Broder, R.G. Compton, The electrochemistry of simple inorganic molecules in room temperature ionic liquids, J. Braz. Chem. Soc. 19 (2008) 611-620.

[41] F. Jalali, S. Riahi, D. Nematollahi, A Kinetic Study on Electrooxidation of PropylThiouracil: An Anti-Hyperthyroid Drug by Potassium Iodide, J. Electrochem. Soc. 160 (2013) H710-H714.

[42] S. Mukherjee, B. Ramalingam, L. Griggs, S. Hamm, G.A. Baker, P. Fraundorf, S. Sengupta, S. Gangopadhyay, Ultrafine sputter-deposited Pt nanoparticles for triiodide reduction in dye-sensitized solar cells: Impact of nanoparticle size, crystallinity and surface coverage on catalytic activity, Nanotechnology. 23 (2012).

[43] C.L. Bentley, A.M. Bond, A.F. Hollenkamp, P.J. Mahon, J. Zhang, Voltammetric Determination of the Iodide/Iodine Formal Potential and Triiodide Stability Constant in Conventional and Ionic Liquid Media, J. Phys. Chem. C. 119 (2015) 22392-22403.

[44] J. Rendl, M. Luster, C. Reiners, Serum inorganic iodide determined by paired-ion reversed-phase HPLC with electrochemical detection, J. Liq. Chromatogr. Relat. Technol. 20 (1997) 1445-1459. 
[45] T.R.I. Cataldi, A. Rubino, M.C. Laviola, R. Ciriello, Comparison of silver, gold and modified platinum electrodes for the electrochemical detection of iodide in urine samples following ion chromatography, J. Chromatogr. B Anal. Technol. Biomed. Life Sci. 827 (2005) 224-231.

[46] C. Bruggink, W.J.M. van Rossum, E. Spijkerman, E.S.E. van Beelen, Iodide analysis by anion-exchange chromatography and pulsed amperometric detection in surface water and adsorbable organic iodide, J. Chromatogr. A. 1144 (2007) 170-174.

[47] M. Cuartero, G.A. Crespo, E. Bakker, Paper-based thin-layer coulometric sensor for halide determination, Anal. Chem. 87 (2015) 1981-1990.

[48] E. Espada-Bellido, Z. Bi, P. Salaün, C.M.G. van den Berg, Determination of iodide and total iodine in estuarine waters by cathodic stripping voltammetry using a vibrating silver amalgam microwire electrode, Talanta. 174 (2017) 165-170.

[49] O.D. Renedo, M.A. Alonso-Lomillo, M.J.A. Martínez, Recent developments in the field of screen-printed electrodes and their related applications, Talanta. 73 (2007) 202219.

[50] M.H. Chiu, W.L. Cheng, G. Muthuraman, C.T. Hsu, H.H. Chung, J.M. Zen, A disposable screen-printed silver strip sensor for single drop analysis of halide in biological samples, Biosens. Bioelectron. 24 (2009) 3008-3013.

[51] J. Bujes-Garrido, D. Izquierdo-Bote, A. Heras, A. Colina, M.J. Arcos-Martínez, Determination of halides using Ag nanoparticles-modified disposable electrodes. A first approach to a wearable sensor for quantification of chloride ions, Anal. Chim. Acta. 1012 (2018) 42-48.

[52] M. Khodari, U. Bilitewski, A.A.H. Basry, Screen-Printed Electrodes for Amperometric Determination of Iodide, Electroanalysis. 27 (2015) 281-284.

[53] A. V. Rzhevskaia, N. V. Shvedene, I. V. Pletnev, Anion-selective electrodes based on solidified 1,3-dihexadecylimidazolium ionic liquids with halide and pseudohalide anions, J. Electroanal. Chem. 783 (2016) 274-279. 
[54] N. V. Shvedene, O.A. Avramenko, V.E. Baulin, L. G. Tomilova, I. V. Pletnev, Iodide-selective screen-printed electrodes based on low-melting ionic solids and metallated phthalocyanine, Electroanalysis. 23 (2011) 1067-1072.

[55] G.C. Ritthidej, Nasal delivery of peptides and proteins with chitosan and related mucoadhesive polymers, in: Pept. Protein Deliv., Elsevier, 2011: pp. 47-68.

[56] S. Mansouri Majd, A. Salimi, B. Astinchap, Label-free attomolar detection of lactate based on radio frequency sputtered of nickel oxide thin film field effect transistor, Biosens. Bioelectron. 92 (2017) 733-740.

[57] G. Wang, J.J. Xu, H.Y. Chen, Z.H. Lu, Amperometric hydrogen peroxide biosensor with sol-gel/chitosan network-like film as immobilization matrix, Biosens. Bioelectron. 18 (2003) 335-343.

[58] Y. Liu, M. Wang, F. Zhao, Z. Xu, S. Dong, The direct electron transfer of glucose oxidase and glucose biosensor based on carbon nanotubes/chitosan matrix, Biosens. Bioelectron. 21 (2005) 984-988.

[59] X. Kang, J. Wang, H. Wu, I.A. Aksay, J. Liu, Y. Lin, Glucose Oxidase-graphenechitosan modified electrode for direct electrochemistry and glucose sensing, Biosens. Bioelectron. 25 (2009) 901-905.

[60] G.D. Altun, S.A. Cetinus, Immobilization of pepsin on chitosan beads, Food Chem. 100 (2007) 964-971.

[61] Ş. Akkuş Çetinus, H. Nursevin Öztop, Immobilization of catalase into chemically crosslinked chitosan beads, Enzyme Microb. Technol. 32 (2003) 889-894.

[62] H. Cunha-Silva, M.J. Arcos-Martinez, Dual range lactate oxidase-based screen printed amperometric biosensor for analysis of lactate in diversified samples, Talanta. 188 (2018) 779-787.

[63] J. Cruz, M. Kawasaki, W. Gorski, Electrode coatings based on chitosan scaffolds, Anal. Chem. 72 (2000) 680-686. 
[64] M. Kumar, A review of chitin and chitosan applications, React. Funct. Polym. 46 (2000) 1-27.

[65] B. Krajewska, Application of chitin- and chitosan-based materials for enzyme immobilizations: A review, Enzyme Microb. Technol. 35 (2004) 126-139.

[66] M. Rinaudo, Chitin and chitosan: Properties and applications, Prog. Polym. Sci. 31 (2006) 603-632.

[67] H. Yajima, M. Morita, M. Hashimoto, H. Sashiwa, T. Kikuchi, T. Ishii, Complex formation of chitosan with iodine and its structure and spectroscopic properties - Molecular assembly and thermal hysteresis behavior, Int. J. Thermophys. 22 (2001) 1265-1283.

[68] K.M. Kim, Y.S. Nam, Y. Lee, K.B. Lee, Highly sensitive and selective assay method for iodide ion determination based on gold nanoparticles conjugated with glycol chitosan, Int. J. Environ. Anal. Chem. 97 (2017) 673-683.

[69] B. Molinero-Abad, D. Izquierdo, L. Pérez, I. Escudero, M.J. Arcos-Martínez, Comparison of backing materials of screen printed electrochemical sensors for direct determination of the sub-nanomolar concentration of lead in seawater, Talanta. 182 (2018) $549-557$.

[70] J. Mocak, A. M. Bond, S. Mitchell, G. Scollary, A statistical overview of standard (IUPAC and ACS) and new procedures for determining the limits of detection and quantification: Application to voltammetric and stripping techniques (Technical Report), Pure Appl. Chem. 69 (1997) 297-328.

[71] E.R. Ziegel, Statistics and Chemometrics for Analytical Chemistry, 2004.

[72] S.A. Bingham, R. Williams, T.J. Cole, C.P. Price, J.H. Cummings, Reference values for analytes of 24-h urine collections known to be complete, Ann. Clin. Biochem. 25 (1988) 610-619. 



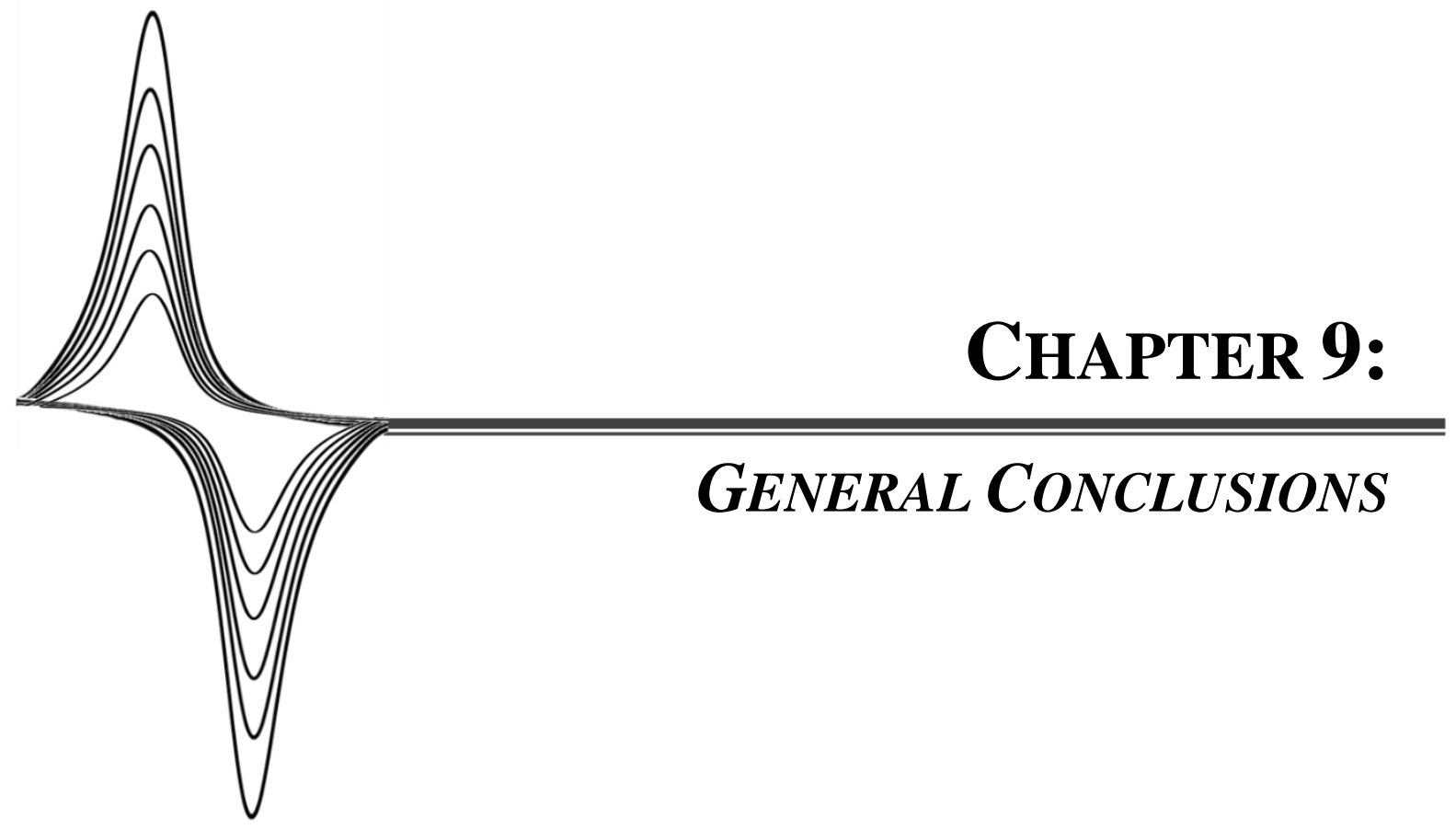



The following conclusions can be drawn from the preceding chapters:

- The versatility and the possibility to easily perform modifications, are the most important characteristics that make the SPEs efficient transducers for biosensing and sensing applications. Additional advantages in terms of the disposability, low-cost, small size and portability for in situ applications, rise the importance of these devices.

- The $\mathrm{LO}_{\mathrm{x}}$-based biosensor, using an SPCE modified with Pt deposit, CS coating, and $\mathrm{Cu}-\mathrm{MOF}$ assisted crosslinking of the enzyme $\left(\mathrm{LO}_{\mathrm{x}}-\mathrm{Cu}-\mathrm{MOF} / \mathrm{CS} / \mathrm{Pt} / \mathrm{SPCE}\right)$ allows to monitoring lactate at $+0.15 \mathrm{~V}$ (vs SPE $\mathrm{Ag} / \mathrm{AgCl}$ ). The biosensor offers improved upper limits of the calibration curves, which are attained with very low number of enzymatic units immobilized in the WE surface.

- The device is the first approach based in a mechanism of substrate inhibition for lactate biosensing. This dual range biosensor is able to be used in catalytic range (from $0.75 \mu \mathrm{M}$ to $1 \mathrm{mM}$ ), and in the inhibition range ( $1 \mathrm{mM}$ to $50 \mathrm{mM}$ ), covering the lactate concentrations of analytical interest.

- The SPCE modification with a Pt deposit, favored the oxidation of hydrogen peroxide produced by $\mathrm{LO}_{\mathrm{x}}$, which occurred at lower potentials than the normally required in amperometric experiments.

- CS plays an important role in $\mathrm{LO}_{\mathrm{x}}$ activity, resulting in higher responses for the lactate biosensing, therefore, being the main contributor in terms of device sensitivity and storage stability (50 days).

- The conjoint crosslinking of $\mathrm{LO}_{\mathrm{x}}$ and $\mathrm{Cu}-\mathrm{MOF}$, assisted with BSA and GA, allowed an efficient orientation of the enzymatic structure on the CS-modified WE, also contributing for the device sensitivity. 
- The $\mathrm{LO}_{\mathrm{x}}$-based biosensor's reproducibility, defined in terms of the RSD\% associated with the slopes of the calibration curves, is $5.03 \%$ for the catalytic zone, and $6.82 \%$ for the inhibition one. A LOD of $0.75 \mu \mathrm{M}$ was obtained using the catalytic zone. The biosensor keeps $90 \%$ of full activity until ten calibration experiments.

- The biosensor proved the suitability to determine lactate in matrices of very different nature, being effective for wines, saliva and sweat analysis.

- The $\mathrm{LO}_{\mathrm{x}}-\mathrm{Cu}-\mathrm{MOF} / \mathrm{CS} / \mathrm{Pt} / \mathrm{SPCE}-$ based biosensor, can be perceived as a proofof-concept for a wearable device, since the sweat lactate range fits ideally in the substrate inhibition zone linearity.

- DLS and fluorescence spectroscopy applied to $\mathrm{LO}_{\mathrm{x}}$ solutions, demonstrated that the enzyme structure suffers conformational changes when submitted to inhibitory concentrations of lactate.

- The amperometric inhibitory response registered for the crosslinked $\mathrm{LO}_{\mathrm{x}}$, outcomes from a pH-dependent mechanism, which is promoted at high substrate concentrations.

- The anodic step carried out at $+1.50 \mathrm{~V}$ (vs SPE $\mathrm{Ag} / \mathrm{AgCl}$ ) during $15 \mathrm{~s}$, using a SPPtE, allows to oxidize the chloride anions of a sample to chlorine gas. After the fast switch between the anodic step and the cathodic potential sweep, of the CSV recorded between $+1.11 \mathrm{~V}$ and $+0.10 \mathrm{~V}$ (vs $\mathrm{SPE} \mathrm{Ag} / \mathrm{AgCl}$ ), the adsorbed chlorine gas is reduced back to chloride anions, developing cathodic peaks at $+0.80 \mathrm{~V}$ (vs SPE $\mathrm{Ag} / \mathrm{AgCl})$.

- The SPPtE-based sensor displays a chloride analysis range, defined between 0.76 $\mathrm{mM}$ and $150 \mathrm{mM}$, which is applicable to several samples. The device is able to perform $118 \pm 5$ reliable experiments, exhibiting a good repeatability. 
- The SPPtE-based sensor presented an RSD\% value associated with the slopes of the calibration curves of $5.80 \%$ and a LOD of $0.76 \mathrm{mM}$ for chloride anion determination.

- The SPPtE-based sensor demonstrate it capability to determine chloride anion in seawater, saline solution, wholemeal biscuits, chicken stock, human plasma and urine.

- The successful transferring of the device as a flexible Gore-Tex ${ }^{\circledR}$ SPPtE-based sensor and the good results obtained in sweat chloride analysis, are both factors contributing as proof-of-concept for the development of a wearable device. Therefore, this sensor can be considered in $\mathrm{CF}$ diagnosis, or to monitoring electrolyte loss in sport activities.

- The application of CSV recorded between $+1.11 \mathrm{~V}$ and $-0.25 \mathrm{~V}$ (vs SPE $\mathrm{Ag} / \mathrm{AgCl}$ ), allowed to obtain chlorine gas (strong oxidative agent) and bromine, both previously generated in the anodic step performed at $+1.20 \mathrm{~V}$ (vs SPE $\mathrm{Ag} / \mathrm{AgCl}$ ) for $20 \mathrm{~s}$, demonstrating an important role in bromide determinations.

- During the potential sweeping of the CSV experiment, chlorine gas promotes the bromine reduction to the corresponding anions, generating quantifiable voltammetric peaks developed at $+0.50 \mathrm{~V}$ (vs SPE $\mathrm{Ag} / \mathrm{AgCl})$.

- The Rh-NPs modified electrodic surface, enhanced the sensor sensitivity to detect bromide, also depleting the chloride contribution in the voltammetric peaks current.

- The Rh/SPCE-based sensor allows a precise and fast analysis of bromide anions over a range up to $40 \mathrm{mM}$, exhibiting an RSD\% value associated with the slopes of the calibration curves of $2.58 \%$ and a LOD of $39 \mu \mathrm{M}$ for the anion determination. 
- A suitable analytical precision for bromide monitoring in seawater, surfactant solutions and pharmaceutical formulations, was attained using the Rh/SPCEbased sensor, which reveals to be a selective and chloride interference-free device.

- After an anodic step performed at $+0.50 \mathrm{~V}$ (vs SPE $\mathrm{Ag} / \mathrm{AgCl}$ ) during $60 \mathrm{~s}$, iodide determination is feasible considering the voltammetric peaks at + $0.05 \mathrm{~V}$ (vs SPE $\mathrm{Ag} / \mathrm{AgCl}$ ), generated by a CSV recorded between $+0.90 \mathrm{~V}$ and $-0.45 \mathrm{~V}$ (vs SPE $\mathrm{Ag} / \mathrm{AgCl})$.

- The application of the CSV at SPCE, allows to achieve an iodide linear analysis range from $5 \mu \mathrm{M}$ to $7.5 \mathrm{mM}$. The SPCE-based iodide sensor's reproducibility, defined in terms of the RSD\% associated with the slopes of the calibration curves, is $2.86 \%$. This device achieved a LOD of $2.9 \mu \mathrm{M}$, therefore, suitable to be used in samples with high content of the halide anion.

- The modification of the SPCE-based sensor with CS, allowed to improve the analytical performance of the device to determine trace concentrations of iodide, exhibiting a lower LOD of $10 \mathrm{nM}$ and an analysis range between 0.15 and 500 $\mu \mathrm{M}$. The CS/SPCE-based sensor exhibited an RSD\% value of $2.76 \%$.

- The validated determination of iodide in real-life samples, proved that both sensors are suitable to perform the analyses, being also an alternative to the existing methods to access the IDDs prevalence. 


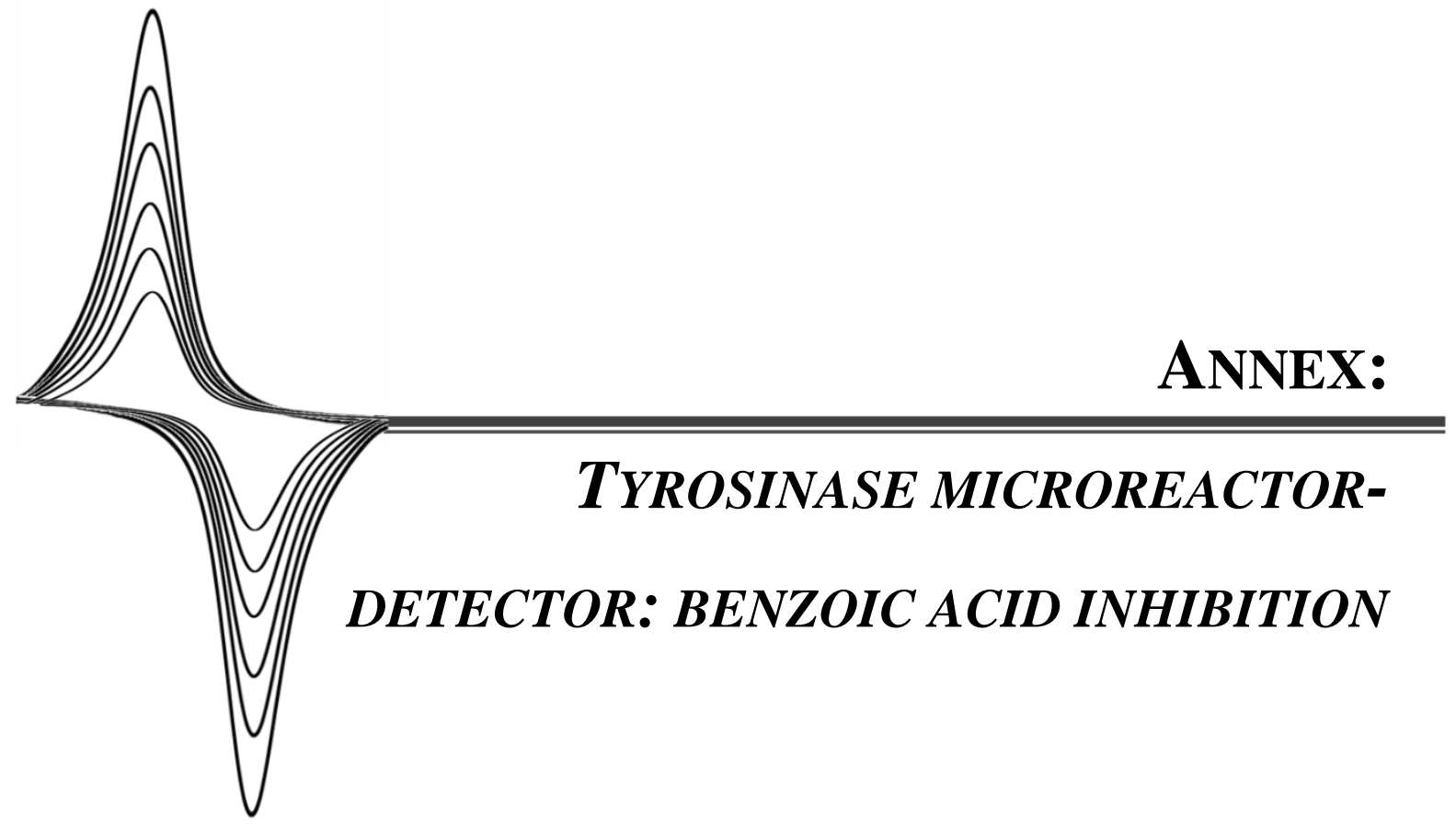



This work arose from an internship financed by the Spanish Ministry of Economy and Competitiveness (EEBB-I-16-11284), which allowed me to work under the supervision of Professor Jean-Michel Kauffmann, at the laboratory of Instrumental Analysis and Bioelectrochemistry of the University Libre de Bruxelles (ULB) (Belgium).

The initial objective of this research internship was to study the $\mathrm{LO}_{\mathrm{x}}$ inhibition, by means of a flow injection analysis system (FIA), to screening which sweat components can act as potential inhibitors of the enzyme activity. Unfortunately, the short time of academic visit and the difficulty to optimize the FIA conditions, led us to change the focus of the research to a more advanced one.

During this internship, my research was focused in the study of molecules that can be potential inhibitors of tyrosinase enzyme (TYR). Using a previously optimized FIA setup for an amperometric microreactor, it was possible to determine the efficiency and the duration of inhibition of two compounds known as TYR inhibitors, namely kojic acid and

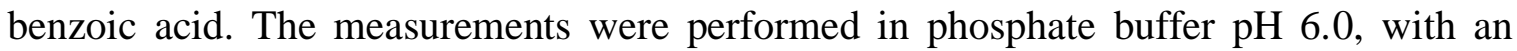
applied potential of $+0.05 \mathrm{~V}(\mathrm{vs} \mathrm{Ag} / \mathrm{AgCl})$ and a flow rate of $100 \mu \mathrm{L} \mathrm{min}^{-1}$. The detector consisted of a gold disk for tyrosinase immobilization adjacent to a glassy carbon working electrode (GCE). Using L-tyrosine as substrate, the enzymatic product formed (dopaquinone) was detected at the GCE, resulting in an amperometric signal, which was used for the screening of tyrosinase inhibitors.

In this annex, are briefly described the results obtained for kojic and benzoic acids as potential inhibitors of TYR activity, which resulted in a collaboration research paper published in Sensors and Actuators B: Chemical 248, 2017, p. 385-394. 



\section{A.1. INTRODUCTION}

Tyrosinase (TYR) is one of the most studied enzymes due to its large interest in food, agriculture, pharmaceutical industries, as well as, in analytical and environmental applications [1,2]. This enzyme catalyzes two distinct oxidation pathways in which molecular oxygen is involved: (i) the monooxygenation of phenols (monophenolase or cresolase activity) and (ii) the oxidation of ortho-diphenols (diphenolase or catecholase activity), both leading to instable ortho-quinone products [1-3]. TYR contains a high conserved binuclear copper in the catalytic center, wherein each copper atom is coordinated to three histidines $[4,5]$.

TYR oxidizes a variety of phenols giving an undesirable brown color in vegetables, fruits and mushrooms [6,7]. In this regard, the identification of inhibitors that modulate TYR activity is of interest in searching of antibrowning agents to preserve the nutritional and visual quality of plant-derived food $[1,2,8]$.

Moreover, TYR is a key enzyme in mammalians melanocytes, where oxidizes the amino acid L-tyrosine into precursors of melanin i.e. dopaquinone (DQ) $[9,10]$. It is sometimes reported that DQ is formed from L-tyrosine via two enzymatically steps: (i) a monophenolase activity where it hydroxylates monophenols (e.g. L-tyrosine) to odiphenols (e.g. L-dopa) and (ii) a diphenolase activity where tyrosinase oxidizes odiphenols to o-quinones (e.g. DQ) (Figure A.1) [8]. Other works suggested that L-tyrosine was monooxigenated into DQ via several intermediaries linked on the copper center of TYR without released of L-dopa molecules [4,11].

In the biochemical process for melanin production, TYR plays a crucial role and can be a potential target for a down regulation to hide unsightly skin disorders [8,9]. TYR inhibitors are often used as whitening and anti-hyperpigment agents in pharmaceutical and cosmetic products. Hydroquinone is the "gold standard" to treat hyperpigmentation, however, 
outcomes in serious side effects. For this reason, the utilization of hydroquinone has been limited to $2.0 \%$ as the maximum of concentration permitted in pharmaceutical formulations. The second most active ingredient on the market as skin lightening agent is kojic acid (KA), a fungal derivate [12]. Even though it is a middle inhibitor, KA is used controversially because of its possible mutagenicity. These compounds suffer from a lack of efficacy and exhibit serious side effects, thus the search for a potent and safe TYR inhibitor to be applied as antibrowning agent, is a continuously ongoing research [13].

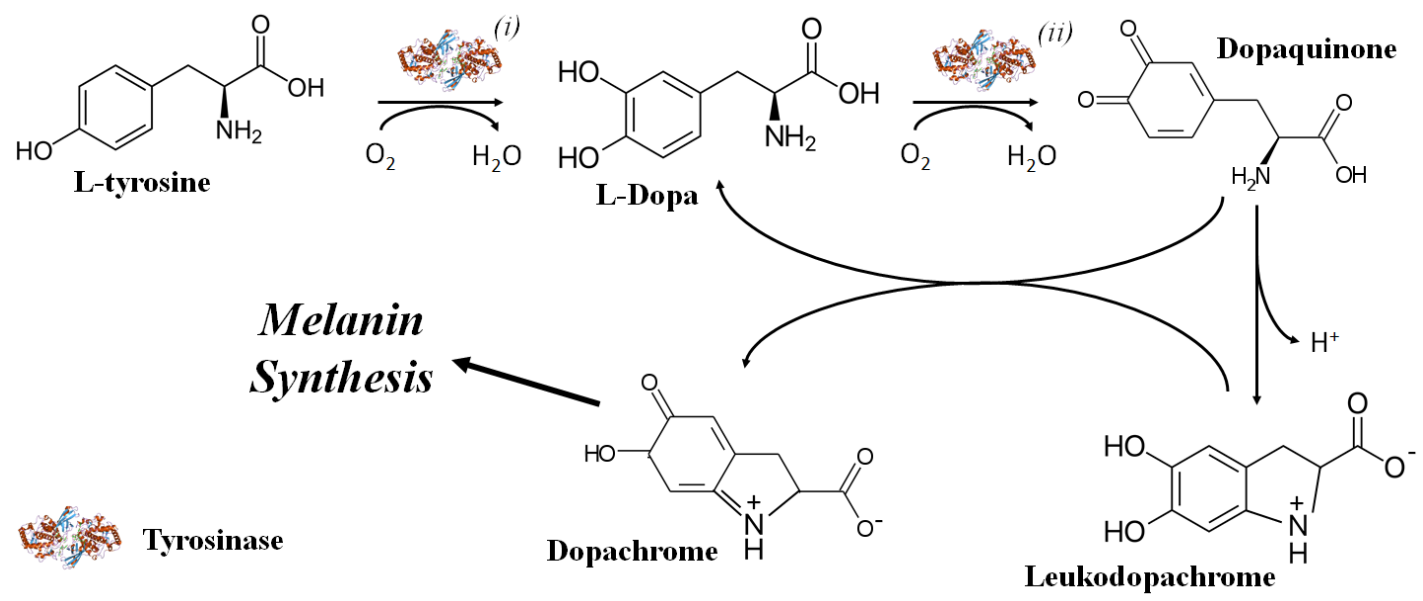

Figure A.1. Simplified biochemical pathway of melanin precursors formation, dependent of TYR activity [8]. (i)TYR monophenolase activity and (ii) TYR diphenolase activity.

Benzoic acid (BA), commonly used to prevent microbial growth and chemical changes in food, beverages, dentifrices, cosmetics and pharmaceuticals products [14], has been recognized to present inhibitory properties for TYR [15-20]. Monophenols are recognized to bind to the oxyform of TYR to one copper ion of the binuclear site via its carboxylate group [20]. BA is believed to function as a monophenol substrate analog, presenting the carboxylate group conjugated to the aromatic ring to bind to the enzyme active site. Indeed, the works regarding the determination of BA using TYR inhibition, reported a competitive inhibition kinetic, binding to the free enzyme rather than enzyme-substrate complex [1520]. However, BA concentration higher than the allowed safety levels, also can be harmful 
to human health, been important to control its potency as TYR inhibitor. Also, is important to compare its inhibitory activity with other molecules, to weigh the efficiency and side effects with the dose required for the final goal [15-17].

It is of interest to develop analytical methods to carry out the TYR inhibitors screening, using a high throughput rate. Spectrophotometric methods are usually applied to evaluate both the monophenolase and diphenolase activities using either L-tyrosine or L-dopa as TYR substrates, with the enzyme in its free form in solution [21-23]. This method is simple, but it is not appropriate for an extensive screening of inhibitors due to enzyme cost [24] and it suffers from a low sensitivity, products instability and from possible interfering species [25].

Systems with TYR immobilized have gained importance in biotechnological applications e.g. synthesis of L-dopa [26] or for the determination of phenol, tyramine and bisphenol A in environmental applications [27-30] and to quantify coliforms [31]. Several TYR biosensors have been described for the determination of enzymatic inhibitors [32-34]. Few of them were also used to screening and compare the inhibitors potency. Screening of inhibitors under hydrodynamic conditions allows a high throughput screening and reusability of the enzyme [35], and also, to determine both inhibition efficiency and enzyme activity recovery rate [36]. The latter is an important criterion to evaluate, since the duration of binding between the inhibitor and the enzyme, also called residence time, is one key information in drug development [37].

In Professor's Kauffmann research group, a thin layer flow-through detector has been customized for the assessment of TYR inhibitors, especially skin whitening agents. The detector with the immobilized enzyme consisted of a gold (Au) disk support upstream, adjacent to a glassy carbon working disk electrode (GCE) located downstream. TYR from mushroom was covalently immobilized onto the Au support pretreated with gold nanoparticles (Au-NPs) and modified with a self-assembled monolayer (SAM) of cysteamine. When injecting L-tyrosine, the enzymatic product (DQ) was detected amperometrically at the GCE. The parameters which were most affecting the amperometric response, were optimized using a central composite design, resulting in a $\mathrm{pH}$ value of 6.0 


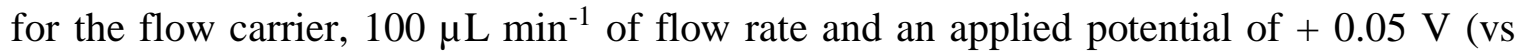
$\mathrm{Ag} / \mathrm{AgCl})[38]$.

The aim of this work was to determine the inhibitor potency and residence time of benzoic acid. The data obtained were linked to previous results obtained, completing a list of a total of twelve potential TYR inhibitors studied.

\section{A.2. EXPERIMENTAL SECTION}

\section{A.2.1. Chemicals and reagents}

L-tyrosine was obtained from Janssen chimica (Beerse, Belgium). Mushroom tyrosinase

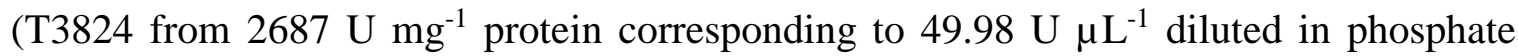
buffer $0.1 \mathrm{M}, \mathrm{pH} 6.8$ ), glutaraldehyde $25 \%$ solution, hydrogen tetrachloroaurate (III) trihydrate, were from Sigma-Aldrich (Bornem, Belgium). Cysteamine hydrochloride and benzoic acid were from Fluka (Neu-Ulm, Germany). Kojic acid was supplied by Kreglinger Europe (Anvers, Belgium).

Phosphate buffer (PB) consisted of a solution of disodium hydrogen phosphate dihydrate $(0.1 \mathrm{M})$ and sodium dihydrogen phosphate dihydrate $(0.1 \mathrm{M})$ to obtain a $\mathrm{pH}$ of 6.0 and filtered through a $0.45 \mu \mathrm{m}$ membrane. L-tyrosine $(0.1 \mathrm{M})$ was dissolved in MilliQ water with a minimal amount of $\mathrm{NaOH}$. Stock solutions of inhibitors $(0.1 \mathrm{M})$ were prepared in MilliQ water for kojic acid, and in MilliQ water with a minimal amount of $\mathrm{NaOH}$ for benzoic acid. The stock solution of studied inhibitors was diluted in PB to appropriate concentration before analysis. 


\section{A.2.2. Apparatus}

Amperometric measurements were realized using a potentiostat LC-4C (BASi, West Lafayette, USA) with a thin-layer flow cell (BASi) consisting of a stainless-steel block as auxiliary electrode and which housed the $\mathrm{Ag} / \mathrm{AgCl}, 3 \mathrm{M} \mathrm{KCl}$ reference electrode. The opposite block contained two metallic disks: it was customized on demand by BASi in order to obtain a gold ( $\mathrm{Au}$ ) disk (diameter $3 \mathrm{~mm}$ ) adjacent to a glassy carbon disk electrode (GCE) (diameter $3 \mathrm{~mm}$ ). The Au disk served only for enzyme immobilization. The flow injection setup consisted of a HPLC pump PM-92E (BASi), a Rheodyne injection valve (20 $\mu \mathrm{L}$ injection loop), and a $150 \mathrm{~cm}$ PEEK tubing (250 $\mu \mathrm{m}$ ID) leading to the flow-through detector. The signal was monitored using a PowerChrom 280 recorder (eDAQ-Europe) which converted units of peak area into $\mathrm{mV} \times \mathrm{s}$. Cyclic voltammetry $(\mathrm{CV})$ was applied for the cleaning of the Au disk and Au-NPs electrodeposition with an Epsilon (BASi).

\section{A.2.3. Tyrosinase microreactor-detector preparation}

The Au disk was cleaned before immobilization. The block consisting of the Au and GCE disks was dipped into an alkaline solution $(0.5 \mathrm{M} \mathrm{KOH})$ and a reductive desorption was applied to completely remove traces of thiol compounds from previous experiments. This was accomplished by connecting the Au disk as working electrode in batch configuration and performing several CVs from $0.0 \mathrm{~V}$ to $-1.3 \mathrm{~V}$, at a scan rate of $100 \mathrm{mVs}^{-1}$. This step was carried out until the peaks at $-0.975 \mathrm{~V}$, due to thiol groups, disappeared. Then, the block was manually smoothed with a fine polishing alumina powder, rinsed with MilliQ water and sonicated for $1 \mathrm{~min}$. Finally, an electrochemical treatment was realized at the Au disk in $\mathrm{H}_{2} \mathrm{SO}_{4}(0.5 \mathrm{M})$ by cycling from $0.0 \mathrm{~V}$ to $+1.5 \mathrm{~V}$ at $100 \mathrm{mV} \mathrm{s}^{-1}$ until baseline stabilization. The pretreatment of the block is presented in figure A.2.(A).

Before enzyme immobilization, the thin layer block was immersed into a $5 \mathrm{mM} \mathrm{HAuCl}_{4}$ solution (prepared in $0.5 \mathrm{M} \mathrm{H}_{2} \mathrm{SO}_{4}$ ) and Au-NPs were electrodeposited, in batch condition, onto the Au disk by applying a negative potential $(-400 \mathrm{mV})$ during $120 \mathrm{~s}$ (Figure A.2.(B)). Afterwards the Au-NPs/Au support was dipped into a $0.1 \mathrm{M}$ cysteamine hydrochloride 
solution overnight in the dark. Finally, a mixture of $10 \mu \mathrm{L}$ of glutaraldehyde solution at $2.5 \%(\mathrm{v} / \mathrm{v})$ (freshly diluted in $0.1 \mathrm{M} \mathrm{PB}, \mathrm{pH}$ of 6.8 ) and $10 \mu \mathrm{L}$ TYR was prepared in an Eppendorf tube. A volume of $4 \mu \mathrm{L}$ of this solution was immediately dropped onto the surface of the gold support and left to dry at room temperature. This step was repeated once more in order to obtain a final volume of $8 \mu \mathrm{L}$ of the mixture deposited onto the cysteamine/Au-NPs/Au disk (Figure A.2.(C)). Between each step of immobilization, the block was washed with Milli-Q water and dried at room temperature. The amperometric microreactor-detector was finally inserted into the FIA setup and stabilized overnight with $\mathrm{PB}$ of $\mathrm{pH} 6.0$ at a flow rate of $50 \mu \mathrm{L} \mathrm{min}^{-1}$ with the GCE at open circuit.

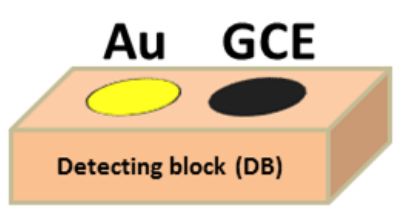

A. Au disk pretreatment

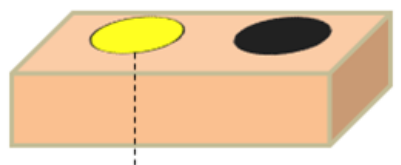

- DB smoothing manually

- $\mathrm{CV}(0 \mathrm{mV} \rightarrow-1300 \mathrm{mV})$ in $\mathrm{KOH} 0.5 \mathrm{M}$

- DB smoothing manually

- $\mathrm{CV}(0 \mathrm{mV} \rightarrow+1500 \mathrm{mV})$ in $\mathrm{H}_{2} \mathrm{SO}_{4} 0.5 \mathrm{M}$

\section{TYR immobilization}

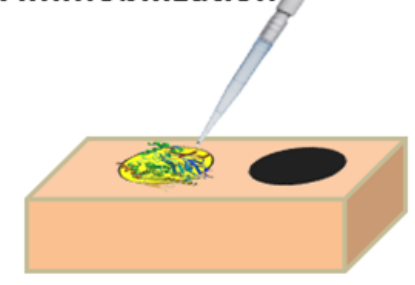

$C Y S T+G A+T Y R$
B. AuNPs electrodeposition

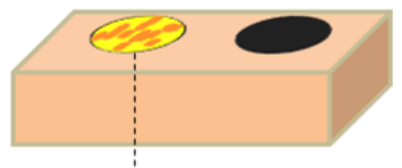

$E_{\text {app }}=-400 \mathrm{mV}, \mathrm{E}_{\text {dep }}=120 \mathrm{~s}, 5 \mathrm{mM} \mathrm{AuCl}_{4}^{-}$

D. Microreactor - detector

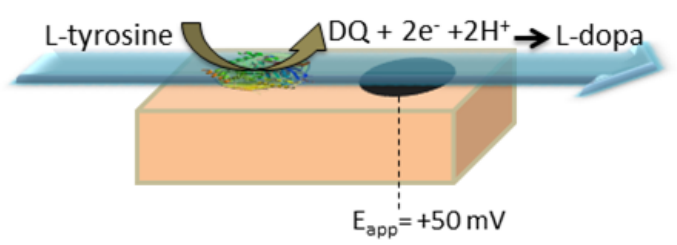

Figure A.2. Schematic drawing of the detecting block $(D B)$ of the flow through microreactordetector. (A) Pretreatment of the Au and GCE disk by cycling the Au support in alkaline and acid solutions and manual polishing of the entire block; (B) electrodeposition of the Au-NPS onto the Au disk; (C) immobilization of tyrosinase (TYR) on the cysteamine (CYST) self-assembled monolayer through the crosslinker glutaraldehyde $(G A)$ and $(D)$ oxidation of L-tyrosine to $D Q$ by TYR and electroreduction of the enzymatic product at the GCE disk (Adapted from [38]). 


\section{A.2.4. Experimental parameters influencing the response}

The TYR microreactor-detector parameters and working conditions were previously optimized to obtain the maximization of the peak area resulting from DQ reduction at the GCE.

L-tyrosine was chosen as TYR substrate instead of L-dopa because it is the starting compound in the melanogenesis process and hence it permitted to study the monophenolase activity of TYR. Moreover, the molecule is more stable than L-dopa and it does not display an electrochemical signal when injected in the FIA system without enzyme immobilized. L-tyrosine is converted into DQ by TYR immobilized at the Au disk and subsequently reduced at adjacent GCE disk to L-Dopa (Figure A.2.(D)).

A non-saturated concentration of L-tyrosine $(1 \mathrm{mM})$ was used in the experiments, which was not too low in order to be able to detect the product generated enzymatically. The first series of substrate injections gave always higher peaks than the subsequent one, therefore it was necessary to realize a pre-conditioning of the system by injecting six times the substrate L-tyrosine $(1 \mathrm{mM})$ before performing the inhibition experiments. This stabilization period was due to a fouling of the GCE by a polymer-like structure coming from the generated DQ.

$\mathrm{An} \mathrm{PB}$ at $\mathrm{pH}$ of 6.0, was found as optimal for the electrochemical signal generation. Moreover, the flow rate was varied to achieve a balance between the duration of interaction of the substrate or the inhibitor with the enzyme for allowing a high throughput screening, resulting in optimal flow rate of $100 \mu \mathrm{L} \mathrm{min}{ }^{-1}$. Finally, the maximized electrochemical signals were obtained for a potential of $+0.05 \mathrm{~V} \mathrm{~s}^{-1}$ ( $\left.\mathrm{vs} \mathrm{Ag} / \mathrm{AgCl}\right)$, applied at the GCE to reduce the generated quinone derivative.

\section{A.2.5. Inhibition measurement}

A typical inhibition experiment consisted in three injections of $1 \mathrm{mM}$ L-tyrosine, followed by one injection of a mixture inhibitor - L-tyrosine (the inhibitor concentration was varied 
while the substrate was maintained at $1 \mathrm{mM}$ ), and finally, 5 injections of $1 \mathrm{mM} \mathrm{L-tyrosine.}$ Injections were realized every 1.30 minutes.

The percentage of inhibition $(I(\%))$ was calculated by comparing the peak area (proportional to the enzymatic product formed) in the absence of inhibitor $\left(\bar{A}_{0}\right.$ average of three measurements) and with the inhibitor $\left(A_{i}\right)$, as expressed previously in equation 3.8 (page 46).

Inhibition curves were obtained by plotting the $I(\%)$ against the inhibitor concentration. Inhibitions lower than $6.0 \%$ were not taken in account. The concentration of inhibitor needed to decrease half of the enzyme activity, i.e. a 50\% decrease of the peak area $\left(I C_{50}\right)$, was determined according to the Lineweaver-Burk curve by plotting the inverse of the $I(\%)$ vs the inverse of inhibitor concentration. The inhibitors tested, KA and BA, were studied at a concentration range from $1 \times 10^{-5}$ to $1 \times 10^{-3} \mathrm{M}$.

\section{A.2.6. Determination of the enzyme recovery rate}

The recovery rate of TYR activity was determined by injecting 3 times L-tyrosine (1 mM) followed by one injection of the mixture L-tyrosine $(1 \mathrm{mM})$ - inhibitor at a concentration of the latter that produced $70 \%$ inhibition i.e. $I C_{70}$ (calculated according to the LineweaverBurk curve by plotting the inverse of the $I(\%)$ vs the inverse of inhibitor concentration). The latter injection was considered as time $(\mathrm{t})=0 \mathrm{~s}$. Finally, L-tyrosine $(1 \mathrm{mM})$ was injected again every $90 \mathrm{~s}$, meaning at time $90,180,270,360$ and $450 \mathrm{~s}$. 


\section{A.3. RESUlTS AND DISCUSSION}

\section{A.3.1. Preliminary results}

The previous experiments of TYR inhibition were performed with a commercially available mushroom TYR batch of $5771 \mathrm{U} \mathrm{mg}^{-1}$ of protein. Since, a new TYR batch (2687 $\mathrm{U} \mathrm{mg}^{-1}$ ) was used to perform the BA study, it was necessary to define a standard to obtain comparable results of both enzymes activity. Since KA had demonstrated an efficient inhibition of TYR in the previous experiments, the KA $I C_{50}$ was used as reference value to compare both enzyme lots. The value of KA $I C_{50}$ obtained for the first batch TYR was of $160 \pm 10 \mu \mathrm{M}$, being expected to obtain a similar result with the new TYR. For that, a KA inhibition study was carried out to determine which concentration leads to the decrease half of the new TYR activity.

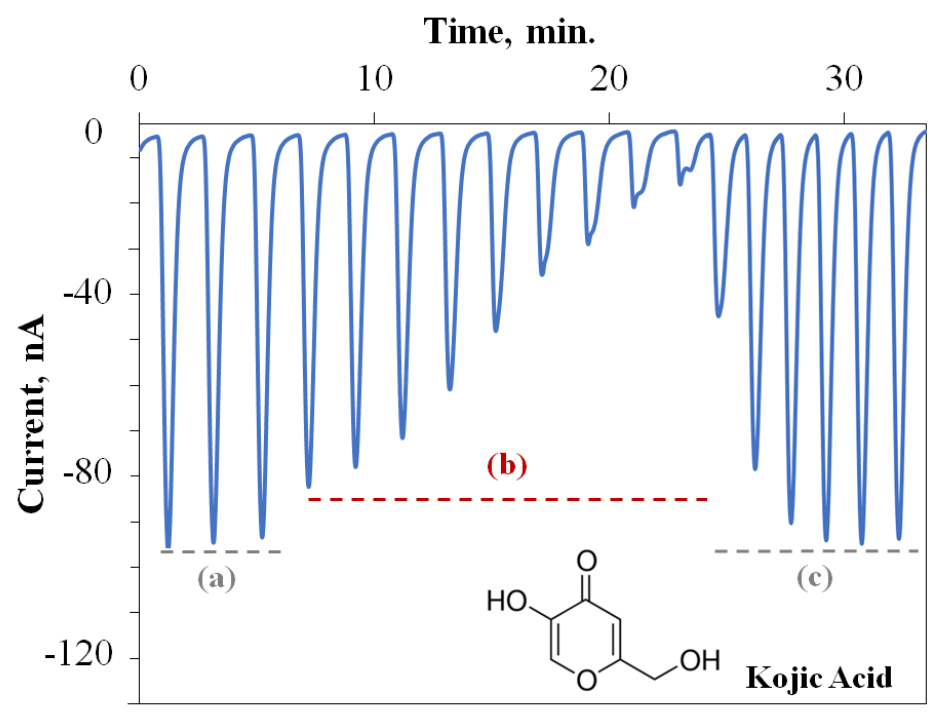

Figure A.3. Typical FIAgram for KA. (a) three repetitive injections of $1 \mathrm{mM}$ L-tyrosine; (b) TYR inhibition through injection of KA concentrations $\left(1.0 \times 10^{-5}, 1.5 \times 10^{-5}, 2.5 \times 10^{-5}, 5.0 \times 10^{-5}, 1.0 \times 10^{-4}\right.$, $\left.2.0 \times 10^{-4}, 3.0 \times 10^{-4}, 6.0 \times 10^{-4}, 1.0 \times 10^{-3} \mathrm{M}\right)+$ L-tyrosine $1 \mathrm{mM} ;(\mathrm{c})$ TYR recovery rate through six injections of L-tyrosine $1 \mathrm{mM}$. 
The determination of the $I(\%)$ was carried out as presented in figure A.3. The FIAgram, shows the typical signals (peak area) corresponding to DQ reduction. The mean of $1 \mathrm{mM}$ L-tyrosine peaks area of three replicates (Figure A.3.(a)), was compared with the peak area obtained by injecting a mixture of $1 \mathrm{mM}$ of L-tyrosine and several KA concentrations $\left(1 \times 10^{-5}\right.$ to $\left.1 \times 10^{-3} \mathrm{M}\right)$ (Figure A.3.(b)). Then, the substrate was injected alone again several times (Figure A.3.(c)).
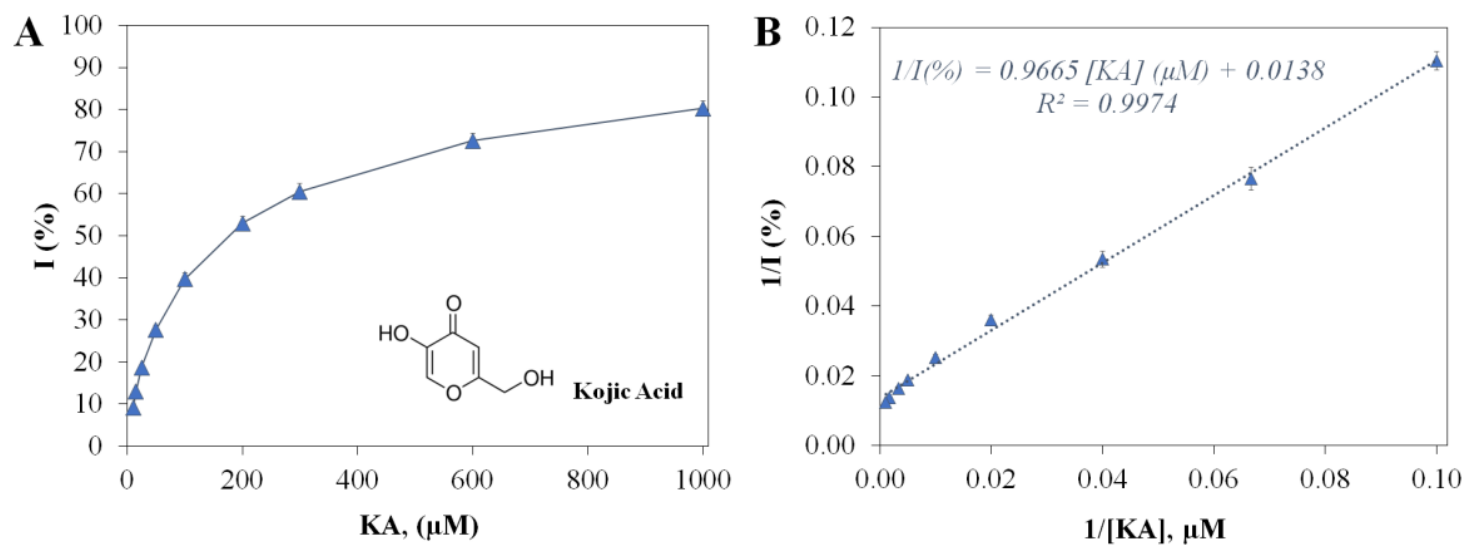

Figure A.4. Typical TYR inhibition curves with KA. (A) inhibition curve obtained for KA studied in the range of $0-1000 \mu \mathrm{M}$ and $(\boldsymbol{B}) \mathrm{KA} I \mathrm{C}_{50}$ determination applying $1 / \mathrm{I}(\%)$ vs $1 /[\mathrm{KA}],(n=3)$.

The inhibition curves were obtained by plotting the $I(\%)$ against the inhibitor concentration (Figure A.4). By applying the Lineweaver-Burk model and representing 1/I(\%) vs 1/[KA], an $I C_{50}$ of $156 \pm 9.90 \mu \mathrm{M}$ was obtained for the new TYR batch, using the KA inhibition.

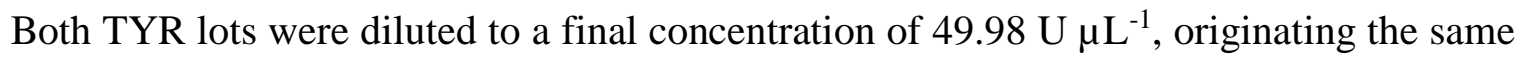
apparent $I C_{50}$ value (Table A.1), meaning that the amount of protein had no influence on the $I(\%)$ observed.

Regarding these results, it is possible to obtain comparative results of TYR inhibition using BA and include the data in the list of twelve compounds, previously studied as potential TYR inhibitors. 


\section{A.3.2. Study of benzoic acid as TYR inhibitor}

The first investigation needed to determine the BA inhibition potency of TYR activity, is to check this monophenol electrochemical behavior onto the microreactor-detector, in order to avoid overlapping currents. Keeping this in mind, an inhibition experiment was performed as previously described in the section A.2.5, with the difference that the inhibitor (BA) was added alone, i.e. without been mixed with L-tyrosine $1 \mathrm{mM}$.

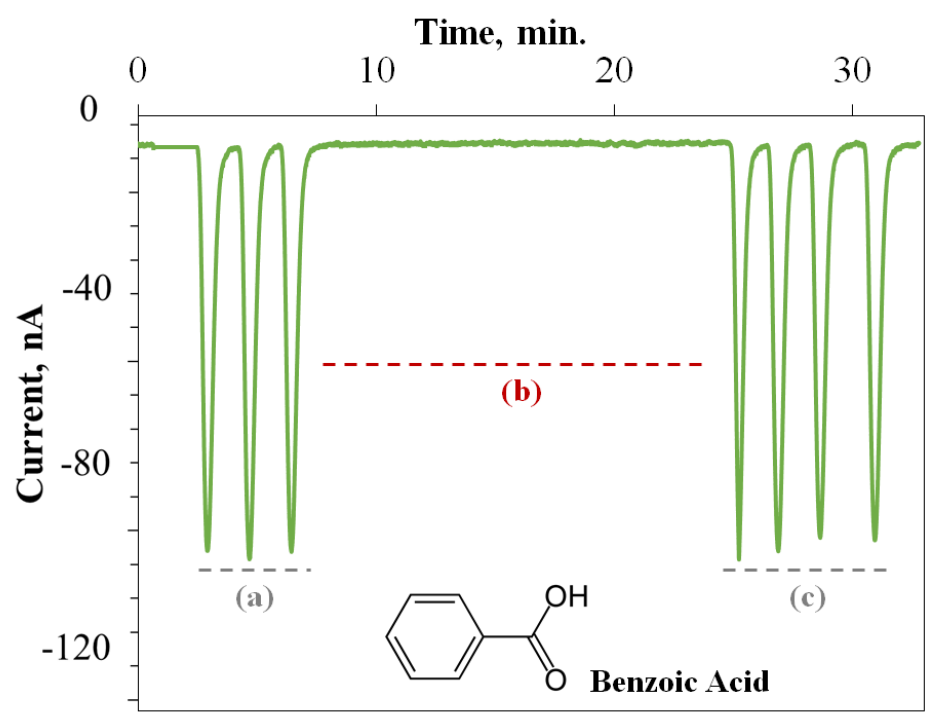

Figure A.5. Screening of BA electrochemical signal. (a) three repetitive injections of $1 \mathrm{mM} \mathrm{L}$ tyrosine; (b) injection of BA concentrations $\left(1.0 \times 10^{-5}, 1.5 \times 10^{-5}, 2.5 \times 10^{-5}, 5.0 \times 10^{-5}, 1.0 \times 10^{-4}\right.$, $\left.2.0 \times 10^{-4}, 3.0 \times 10^{-4}, 6.0 \times 10^{-4}, 1.0 \times 10^{-3} \mathrm{M}\right) ;(\mathrm{c})$ Injections of $1 \mathrm{mM}$ L-tyrosine.

As shown in figure A.5, the first three injections of L-tyrosine resulted in three reduction peaks (Figure A.5.(a)), revealing that TYR is catalyzing the substrate to DQ, which is which is reduced in the adjacent GCE. After this, several injections of BA concentrations, shows to have no effect in the electrochemical signal, developing a stable base line (Figure A.5.(b)). Finally, several injections of L-tyrosine were performed to confirm that the TYR is active and that the results obtained are valid (Figure A.5.(c)). This indicate that such molecule can be applied in the microreactor-detector for TYR inhibition studies, without electrochemical signal contribution. 


\section{A.3.3. Inhibitor potency and $I_{50}$ determination for benzoic acid}

The determination of the $I(\%)$ was carried out as previously described for KA (Section A.3.1.). After three $1 \mathrm{mM}$ L-tyrosine injections onto the microreactor-detector, the average values of substrate peaks area (Figure A.6.(a)) was compared to the peak area obtained by injecting a mixture of the substrate and the inhibitor (Figure A.6.(b)).

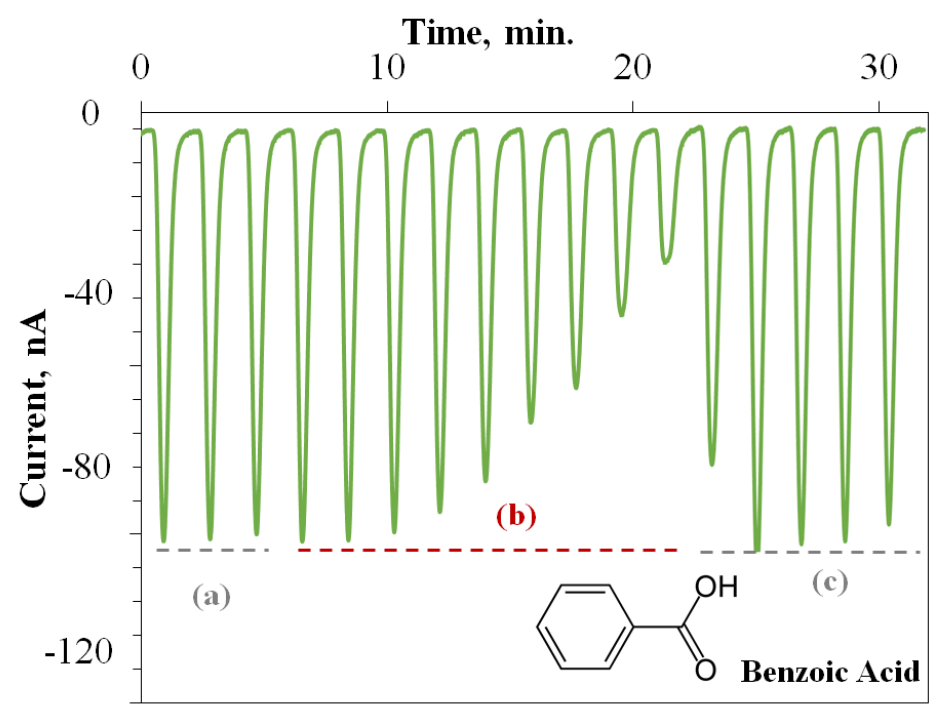

Figure A.6. Typical FIAgram for BA. (a) three repetitive injections of $1 \mathrm{mM}$ L-tyrosine; (b) TYR inhibition through injection of BA concentrations $\left(1.0 \times 10^{-5}, 1.5 \times 10^{-5}, 2.5 \times 10^{-5}, 5.0 \times 10^{-5}, 1.0 \times 10^{-4}\right.$, $\left.2.0 \times 10^{-4}, 3.0 \times 10^{-4}, 6.0 \times 10^{-4}, 1.0 \times 10^{-3} \mathrm{M}\right)+1 \mathrm{mM}$ L-tyrosine; (c) TYR recovery rate through six injections of $1 \mathrm{mM}$ L-tyrosine.

Comparing the FIAgrams obtained with KA (Figure A.3) and BA (Figure A.6), KA proves to be a stronger TYR inhibitor. In fact, BA is described to be a weak tyrosinase inhibitor, which agrees with the behavior found [5]. At the lowest BA concentrations tested, 1.0 $\times 10^{-}$ ${ }^{5}, 1.5 \times 10^{-5}$ and $2.5 \times 10^{-5} \mathrm{M}$, the TYR inhibition was unnoticeable exhibiting an activity near of $100 \%$. In KA assay, when at the same concentrations, the inhibitor outcomes in a notorious decay in the enzyme activity. Still, higher concentrations of BA promote a TYR inhibition, allowing to study its potency, the respective $I C_{50}$ and inhibitor dynamic binding (residence time). 
A typical inhibition curve was obtained for BA (Figure A.7.(A)). BA present a mechanism known to chelate the copper inside the enzymatic catalytic site. Also, KA is recognized to use this inhibition mechanism [39-41]. Both are called "true inhibitors" because they directly interact with the enzyme [42].

For the calculation of BA $I C_{50}$, only the peaks obtained for concentrations higher than $2.5 \times 10^{-5} \mathrm{M}$ were considered, since previous concentrations shows an insignificant variation of the enzymatic activity. As shown in figure A.7.(B), by using the Lineweaver-Burk linearization of $1 / I(\%)$ vs $1 /[\mathrm{BA}]$, it was possible to determine an $I C_{50}$ of $767 \pm 60.0 \mu \mathrm{M}$ for BA TYR inhibition.
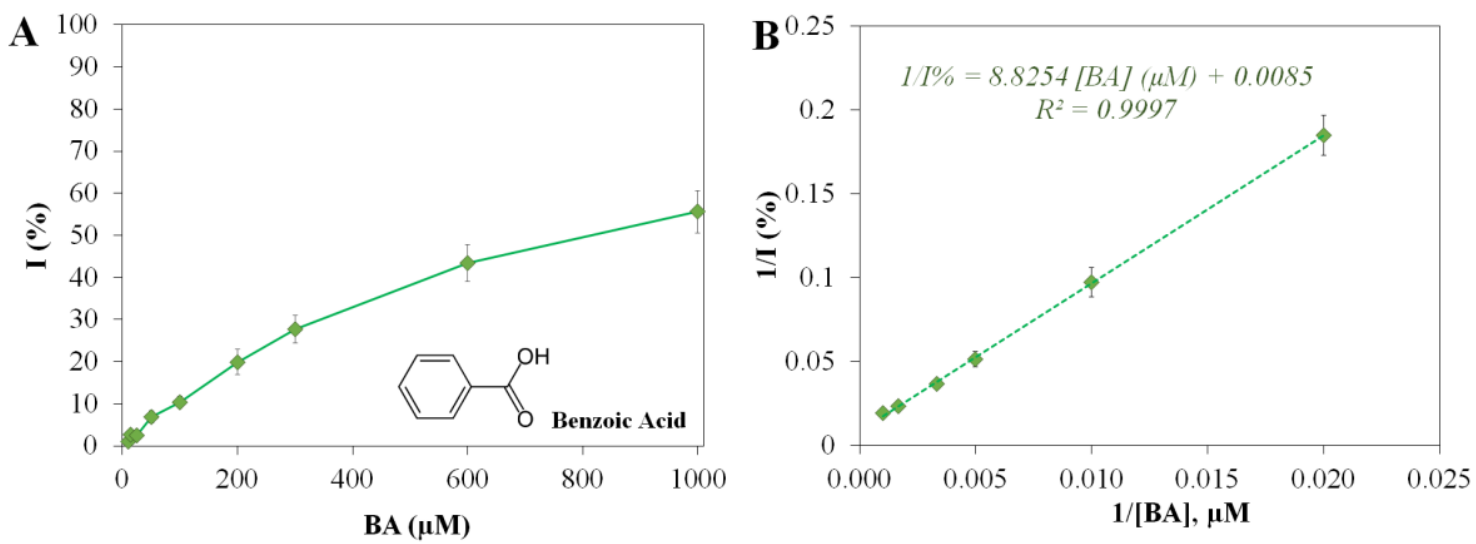

Figure A.7. Typical TYR inhibition curves with BA. (A) inhibition curve obtained for BA studied in the range of $0-1000 \mu M$ and $(\boldsymbol{B}) B A I_{50}$ determination applying $1 / I(\%)$ vs $1 /[B A],(n=3)$.

Regarding KA and BA $I C_{50}$ values (Table A.1), TYR inhibition through BA requires about 5 times more inhibitor concentration than KA. Similarly, to the observed with other molecules an excess of BA can result in side effects to the human health [17], which had no advantages to the use of other inhibitors. However, if this molecule has a slow binding dynamic, i.e. if it remains in TYR active center for more time than other molecules, even at lower concentrations, it can be contemplated as a potential target for pharmaceutical 
formulations. For that reason, it is important to have the full knowledge about the reactivation of TYR and to know the BA residence time.

Table A.1. $I C_{50}$ of $K A$ and $B A$ (results are expressed as the means $\pm S D, n=3$ ).

\begin{tabular}{ccc}
\hline Inhibitors & \multicolumn{2}{c}{ Apparent IC50 $(\boldsymbol{\mu M})$ microreactor-detector } \\
& TYR Batch $5771 \mathrm{U} \mathrm{mg}^{-1}$ & TYR Batch $2687 \mathrm{U} \mathrm{mg}^{-1}$ \\
\hline Kojic Acid & $160 \pm 10.0$ & $156 \pm 9.90$ \\
Benzoic Acid & - & $767 \pm 60.0$ \\
\hline
\end{tabular}

\section{A.3.4. TYR reactivation after benzoic acid inhibition}

The stability of inhibitor-enzyme structure is an important parameter in selecting good inhibitor candidates [37]. The developed setup permitted to study this criteria in-line under hydrodynamic conditions. To study the reactivation of the enzyme, an inhibition assay was performed at the $I C_{70}$ of the inhibitor calculated according to Lineweaver-Burk curve. Using the equation $1 / I(\%)=8.82541 /[B A](\mu M)+0.0085$, a BA $I C_{70}$ of $\sim 1525 \mu \mathrm{M}$ was obtained. This $I C_{70}$ value was then used to perform the TYR reactivation assays (Figure A.8. A(b)).

The experiments consisted to inject L-tyrosine $(1 \mathrm{mM})$ every $90 \mathrm{~s}$ after the simultaneously injection inhibitor-substrate (Figure A.8.A(c)). It was found that, $90 \mathrm{~s}$ after the BA inhibition, the enzyme was reactivated to $78 \%$, exhibiting a fast recovery of its activity with $100 \%$ after $180 \mathrm{~s}$ (Figure A.8.(B)). This low residence time of BA, confirm that this monophenol is rapidly substituted by L-tyrosine onto the TYR active center, originating a reversible inhibition mechanism. Interestingly, at $270 \mathrm{~s}$, L-tyrosine injection lead to a decrease to $90 \%$ of the initial TYR activity, achieving later a stabilization. This may be related with the enzyme stability, more than an inhibition effect, as is shown in the following section A.3.6. 

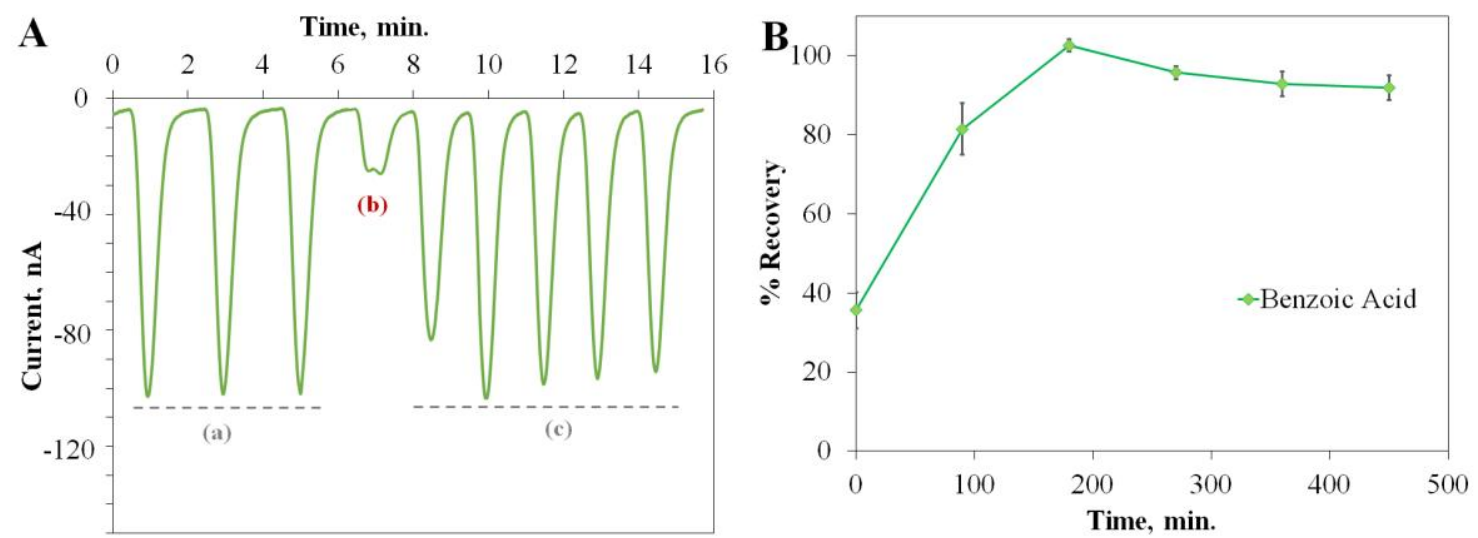

Figure A.8. TYR reactivation assay after BA inhibition. A - FIAgram for BA: (a) three repetitive injections of $1 \mathrm{mM}$ L-tyrosine; (b) injection of $B A I C_{70}(1525 \mu M)+1 \mathrm{mM}$ L-tyrosine; (c) TYR recovery rate through five injections of $1 \mathrm{mM} L$-tyrosine.

\section{A.3.5. Inhibition peaks splitting}

A splitting of the reduction peaks was observed during the simultaneous injection of Ltyrosine and highest inhibitors concentrations tested (figures A.3 and figure A.8.(A)). Using the KA system to investigate why these two peaks appeared, the flow rate was varied and a fixed concentration of $\mathrm{KA}(1 \mathrm{mM})$ and L-tyrosine $(1 \mathrm{mM})$ mixture was injected. A higher resolution of the two peaks was obtained at a flow rate of $25 \mu \mathrm{L} \mathrm{min}{ }^{-1}$.

After defining the flow rate, the concentration of L-tyrosine was varied from 0.25 to $6 \mathrm{mM}$ and the KA concentration was maintained at $1 \mathrm{mM}$ (Figure A.9). The peak duplication shows to be independent of the substrate concentrations, since it is developed for all injections of the mixture L-tyrosine with $1 \mathrm{mM}$ of KA. Previous studies shown that is only possible to obtain a single peak in presence of $0.05 \mathrm{mM} \mathrm{KA} \mathrm{[38].} \mathrm{Thus,} \mathrm{it} \mathrm{was} \mathrm{inferred} \mathrm{that}$ the inhibitors likely reacted with the enzyme with different strengths. Inhibitor weak interaction is washed away under the hydrodynamic conditions, leading to the re-access of the substrate, which results in the formation of the post peaks. 


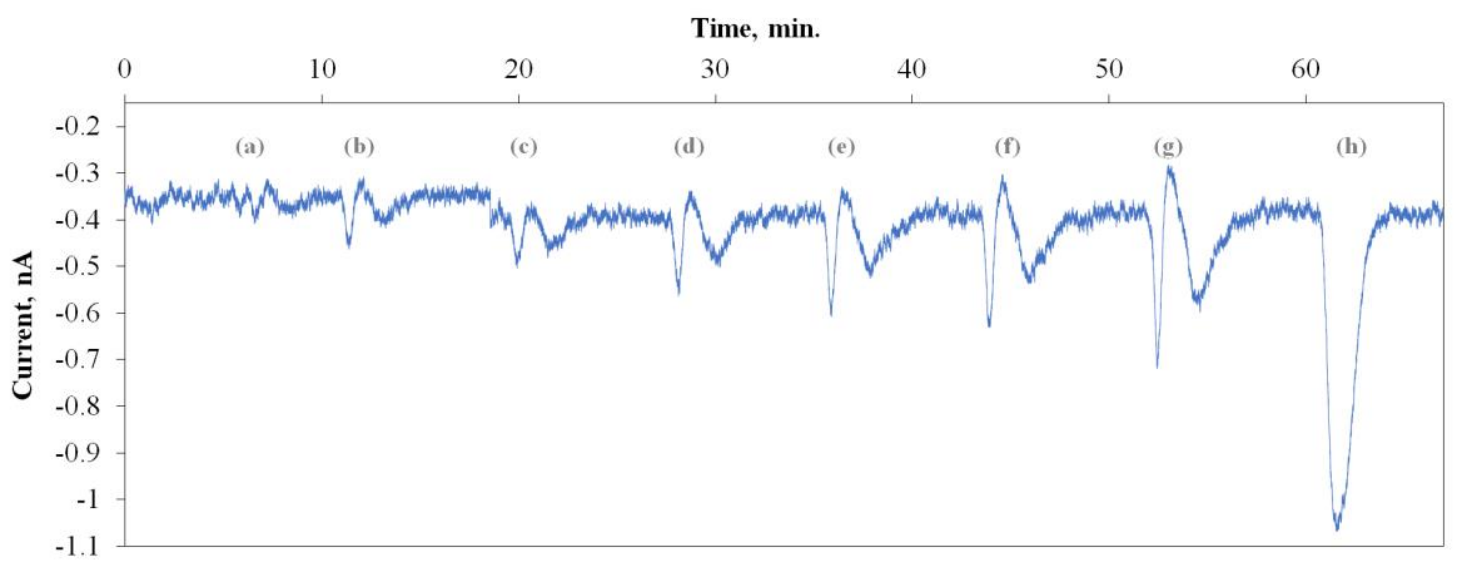

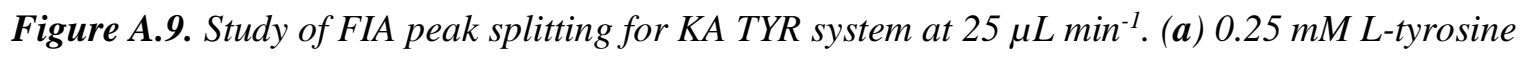
$+1 \mathrm{mM} \mathrm{KA}$, (b) $0.5 \mathrm{mM}$ L-tyrosine $+1 \mathrm{mM} \mathrm{KA}$, (c) $1 \mathrm{mM}$ L-tyrosine + $1 \mathrm{mM} \mathrm{KA}$, (d) $2 \mathrm{mML}$ tyrosine + $1 \mathrm{mMKA}$, (e) $3 \mathrm{mM}$ L-tyrosine + $1 \mathrm{mM} \mathrm{KA}$, (f) $4 \mathrm{mML}$-tyrosine + $1 \mathrm{mM} \mathrm{KA},(\mathrm{g}) 6 \mathrm{mM}$ L-tyrosine $+1 \mathrm{mMKA}$, (h) $1 \mathrm{mM}$ L-tyrosine.

\section{A.3.6. Microreactor-detector stability over time}

The stability of the system was evaluated daily, regarding the response of TYR to $1 \mathrm{mM}$ of L-tyrosine, injected three times before the inhibitors assays $(n=3)$ (Figure A.10). It was observed that during the first days, the amperometric signal of the enzymatic product formed decreased quickly and then slowly, after which achieve a near steady state.

A response was still recorded after 20 days of TYR immobilization at the microreactordetector. However, the previous experiments, determined that the $I(\%)$ for $0.1 \mathrm{mM} \mathrm{KA}$ increased, meaning that the enzyme was more sensitive to inhibition when it exhibited less activity [38]. Thus, in order to have a reliable $I(\%)$ and, consequently, a reliable apparent $I C_{50}$, the immobilized enzyme had to be changed after approximately 60 inhibitor injections. The $I(\%)$ of $\mathrm{KA}(0.1 \mathrm{mM}$ and $0.3 \mathrm{mM})$ was not significantly different during 6 days of operational use despite the enzymatic decreasing activity. 


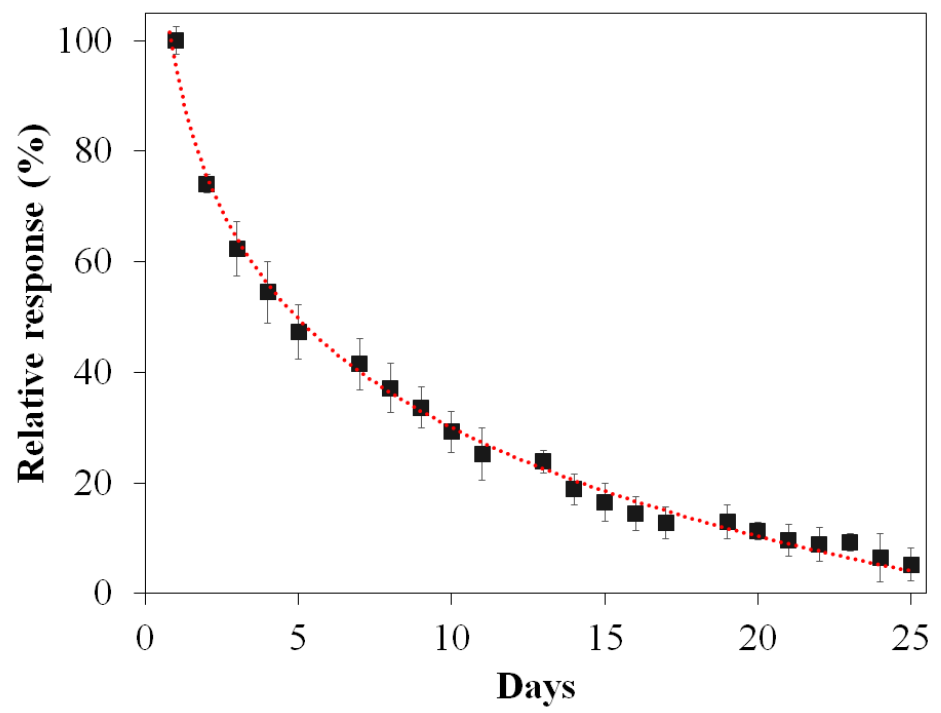

Figure A.10. Operational stability of the immobilized TYR over the days. L-tyrosine $1 \mathrm{mM}$ relative response. (Results expressed as the mean, $n=3$ ).

\section{A.4. Conclusions}

A FIA setup with a self-contained flow-through microreactor-detector with immobilized tyrosinase and an amperometric detector was applied to study benzoic acid as inhibitor molecule of the tyrosinase activity. The optimized parameters allowed to study the inhibition potency (apparent $I C_{50}$ ) and mechanism. Moreover, of particular interest and in addition to the strength of inhibition, this bioanalytical tool allowed to investigate the kinetic of enzyme activity recovery, an important criterion when searching for new drug inhibitors. The developed method provided additional information regarding the mechanism and dynamics of tyrosinase inhibition.

Despite no advantages were found for BA between the studied compounds for TYR inhibition, the microreactor-detector provides useful information regarding the mechanism and dynamics of this enzyme inhibition. 


\section{A.5. REFERENCES}

[1] K.R.S. Sambasiva Rao, N.K. Tripathy, D. Srinivasa Rao, R.S. Prakasham, Production, characterization, catalytic and inhibitory activities of tyrosinase, Res. J. Biotechnol. 8 (2013) 87-99.

[2] P. Agarwal, S. Saroj, S. Dubey, R. P Singh, L-tyrosinase - A multifunctional enzyme structural and molecular features in Biotechnology, Volume 5: Gene and Protein Engineering, Studium Press LLC, New Delhi, 2014.

[3] S.-Y. Seo, V.K. Sharma, N. Sharma, Mushroom tyrosinase: recent prospects, J. Agric. Food Chem. 51 (2003) 2837-2853.

[4] C.A. Ramsden, P.A. Riley, Tyrosinase: the four oxidation states of the active site and their relevance to enzymatic activation, oxidation and inactivation., Bioorg. Med. Chem. 22 (2014) 2388-2395.

[5] Y.-J. Kim, H. Uyama, Tyrosinase inhibitors from natural and synthetic sources: structure, inhibition mechanism and perspective for the future, Cell. Mol. Life Sci. 62 (2005) 1707-1723.

[6] I. Kubo, Q.-X. Chen, K. Nihei, Molecular design of antibrowning agents: antioxidative tyrosinase inhibitors, Food Chem. 81 (2003) 241-247.

[7] M.R. Loizzo, R. Tundis, F. Menichini, Natural and synthetic tyrosinase inhibitors as antibrowning agents: an update, Compr. Rev. Food Sci. Food Saf. 11 (2012) 378-398.

[8] S. Zolghadri, A. Bahrami, M.T. Hassan Khan, J. Munoz-Munoz, F. Garcia-Molina, F. Garcia-Canovas, A.A. Saboury, A comprehensive review on tyrosinase inhibitors, J. Enzyme Inhib. Med. Chem. 34 (2019) 279-309. 
[9] T.-S. Chang, Natural melanogenesis inhibitors acting through the down-regulation of tyrosinase activity, Materials (Basel). 5 (2012) 1661-1685.

[10] S. Parvez, M. Kang, H.-S. Chung, H. Bae, Naturally occurring tyrosinase inhibitors: mechanism and applications in skin health, cosmetics and agriculture industries, Phytother. Res. 21 (2007) 805-816.

[11] C.A. Ramsden, P.A. Riley, Mechanistic studies of tyrosinase suicide inactivation, Spec. Issue Rev. Accounts Ark. (2010) 260-274.

[12] Z.D. Draelos, Skin lightening preparations and the hydroquinone controversy, Dermatol. Ther. 20 (2007) 308-313.

[13] F. Solano, S. Briganti, M. Picardo, G. Ghanem, Hypopigmenting agents: an updated review on biological, chemical and clinical aspects, Pigment Cell Res. 19 (2006) 550-571.

[14] A.S. Abedi, A. Mohammadi, E. Azadniya, A.M. Mortazavian, R. Khaksar, Simultaneous determination of sorbic and benzoic acids in milk products using an optimised microextraction technique followed by gas chromatography, Food Addit. Contam. - Part A Chem. Anal. Control. Expo. Risk Assess. 31 (2014) 21-28.

[15] M.K. Sezgintürk, T. Göktuğ, E. Dinçkaya, Detection of benzoic acid by an amperometric inhibitor biosensor based on mushroom tissue homogenate, Food Technol. Biotechnol. 43 (2005) 329-334.

[16] J. Kochana, J. Kozak, A. Skrobisz, M. Woźniakiewicz, Tyrosinase biosensor for benzoic acid inhibition-based determination with the use of a flow-batch monosegmented sequential injection system, Talanta. 96 (2012) 147-152.

[17] M.D. Morales, S. Morante, A. Escarpa, M.C. González, A.J. Reviejo, J.M. Pingarrón, Design of a composite amperometric enzyme electrode for the control of the benzoic acid content in food, Talanta. 57 (2002) 1189-1198. 
[18] S. Li, Y. Tan, P. Wang, J. Kan, Inhibition of benzoic acid on the polyanilinepolyphenol oxidase biosensor, Sensors Actuators, B Chem. 144 (2010) 18-22.

[19] V.H. Sima, S. Patris, Z. Aydogmus, A. Sarakbi, R. Sandulescu, J.-M. Kauffmann, Tyrosinase immobilized magnetic nanobeads for the amperometric assay of enzyme inhibitors: application to the skin whitening agents, Talanta. 83 (2011) 980-987.

[20] S. Menon, R.W. Fleck, G. Yong, K.G. Strothkamp, Benzoic acid inhibition of the alpha, beta, and gamma isozymes of Agaricus bisporus tyrosinase., Arch. Biochem. Biophys. 280 (1990) 27-32.

[21] I. Behbahani, S.A. Miller, D.H. Okeeffe, A comparison of mushroom tyrosinase dopaquinone and dopachrome assays using diode-array spectrophotometry: dopachrome formation vs ascorbate-linked dopaquinone reduction, Microchem. J. 47 (1993) 251-260.

[22] Y.S. Lee, J.H. Park, M.H. Kim, S.H. Seo, H.J. Kim, Synthesis of tyrosinase inhibitory kojic acid derivative, Arch. Pharm. (Weinheim). 339 (2006) 111-114.

[23] S. Nithitanakool, P. Pithayanukul, R. Bavovada, P. Saparpakorn, Molecular docking studies and anti-tyrosinase activity of Thai mango seed kernel extract., Molecules. 14 (2009) 257-265.

[24] K.B. de Oliveira, K.L. Mischiatti, J.D. Fontana, B.H. de Oliveira, Tyrosinase immobilized enzyme reactor: development and evaluation, J. Chromatogr. B. 945 (2014) $10-16$.

[25] K. Haghbeen, E. Wue Tan, Direct spectrophotometric assay of monooxygenase and oxidase activities of mushroom tyrosinase in the presence of synthetic and natural substrates, Anal. Biochem. 312 (2003) 23-32.

[26] S. Tuncagil, S.K. Kayahan, G. Bayramoglu, M.Y. Arica, L. Toppare, 1-Dopa synthesis using tyrosinase immobilized on magnetic beads, J. Mol. Catal. B Enzym. 58 (2009) 187-193. 
[27] M.N. Karim, H.J. Lee, Amperometric phenol biosensor based on covalent immobilization of tyrosinase on Au nanoparticle modified screen printed carbon electrodes, Talanta. 116 (2013) 991-996.

[28] V. Carralero Sanz, M.L. Mena, A. González-Cortés, P. Yáñez-Sedeño, J.M. Pingarrón, Development of a tyrosinase biosensor based on gold nanoparticles-modified glassy carbon electrodes, Anal. Chim. Acta. 528 (2005) 1-8.

[29] I.M. Apetrei, C. Apetrei, Amperometric biosensor based on polypyrrole and tyrosinase for the detection of tyramine in food samples, Sensors Actuators B Chem. 178 (2013) 40-46.

[30] L. Wu, D. Deng, J. Jin, X. Lu, J. Chen, Nanographene-based tyrosinase biosensor for rapid detection of bisphenol A, Biosens. Bioelectron. 35 (2012) 193-199.

[31] Y. Cheng, Y. Liu, J. Huang, K. Li, Y. Xian, W. Zhang, L. Jin, Amperometric tyrosinase biosensor based on $\mathrm{Fe}_{3} \mathrm{O}_{4}$ nanoparticles-coated carbon nanotubes nanocomposite for rapid detection of coliforms, Electrochim. Acta. 54 (2009) 2588-2594.

[32] D. Shan, Q. Li, H. Xue, S. Cosnier, A highly reversible and sensitive tyrosinase inhibition-based amperometric biosensor for benzoic acid monitoring, Sensors Actuators B Chem. 134 (2008) 1016-1021.

[33] E. Asav, E. Yorganci, E. Akyilmaz, An inhibition type amperometric biosensor based on tyrosinase enzyme for fluoride determination, Talanta. 78 (2009) 553-556.

[34] L. Campanella, D. Lelo, E. Martini, M. Tomassetti, Organophosphorus and carbamate pesticide analysis using an inhibition tyrosinase organic phase enzyme sensor; comparison by butyrylcholinesterase+choline oxidase opee and application to natural waters, Anal. Chim. Acta. 587 (2007) 22-32.

[35] Y. Nie, W. Wang, Immobilized Enzyme Reactor in On-line LC and Its Application in Drug Screening, Chromatogr. Suppl. 69 (2009) S5-S12. 
[36] M. Vandeput, C. Parsajoo, J. Vanheuverzwijn, S. Patris, Y. Yardim, A. le Jeune, A. Sarakbi, D. Mertens, J.-M. Kauffmann, Flow-through enzyme immobilized amperometric detector for the rapid screening of acetylcholinesterase inhibitors by flow injection analysis., J. Pharm. Biomed. Anal. 102 (2015) 267-275.

[37] S. Borman, Residence Time Advances, Chem. Eng. News. 93 (2015) 36-37.

[38] M. Vandeput, S. Patris, H. Silva, C. Parsajoo, B. Dejaeghere, J.A. Martinez, J.M. Kauffmann, Application of a tyrosinase microreactor - detector in a flow injection configuration for the determination of affinity and dynamics of inhibitor binding, Sensors Actuators, B Chem. 248 (2017) 385-394.

[39] S. Briganti, E. Camera, M. Picardo, Chemical and instrumental approaches to treat hyperpigmentation, Pigment Cell Res. 16 (2003) 101-110.

[40] F.S. Senol, M.T.H. Khan, G. Orhan, E. Gurkas, I.E. Orhan, N.S. Oztekin, F. Ak, In silico approach to inhibition of tyrosinase by ascorbic acid using molecular docking simulations, Curr. Top. Med. Chem. 14 (2014) 1469-1472.

[41] S. Menon, R.W. Fleck, G. Yong, K.G. Strothkamp, Benzoic acid inhibition of the $\alpha$, $\beta$, and $\gamma$ Isozymes of Agaricus bisporus tyrosinase, Arch. Biochem. Biophys. 280 (1990) $27-32$.

[42] T.-S. Chang, An updated review of tyrosinase inhibitors., Int. J. Mol. Sci. 10 (2009) 2440-2475. 